\title{
Fundamental Understanding of Ambient and High-Temperature Plasticity Phenomena in Structural Materials in Advanced Reactors
}

\section{Fuel Cycle R\&D}

Dr. Chaitanya Deo Georgia Institute of Technology

Sue Lesica, Federal POC Cetin Unal, Technical POC

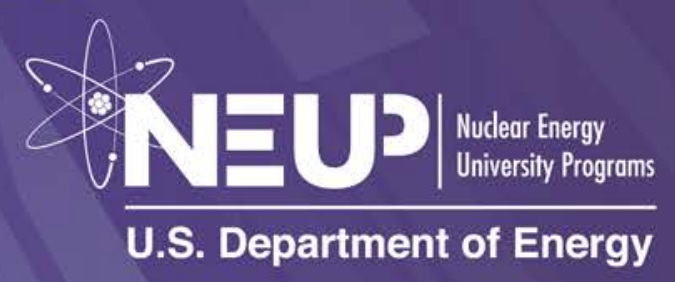




\title{
NEUP Final Report
}

\section{Fundamental understanding of ambient and high temperature plasticity phenomena in structural materials in advanced reactors}

\author{
PI: Dr. Chaitanya Deo, Georgia Institute of Technology, Atlanta, GA \\ Co-PIs: Drs. David McDowell and Ting Zhu, Georgia Institute of Technology, Atlanta, GA \\ Proposal ID: 09-806 \\ Tracking ID: CFP-09-269
}

\section{Contents}

Fundamental understanding of ambient and high temperature plasticity phenomena in structural

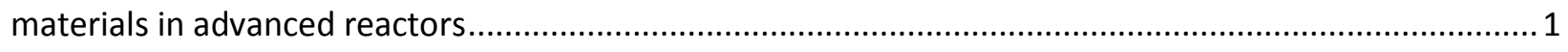

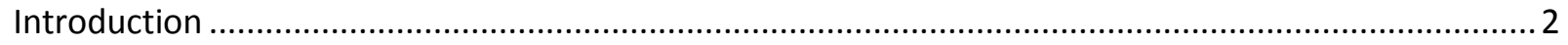

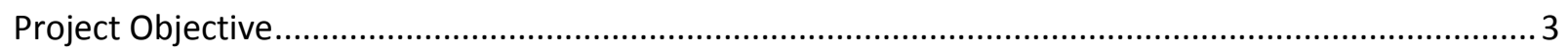

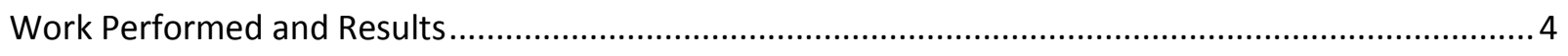

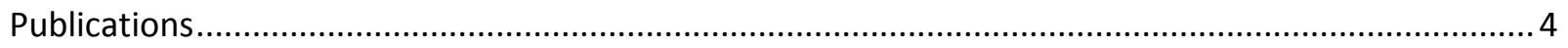

Presentations

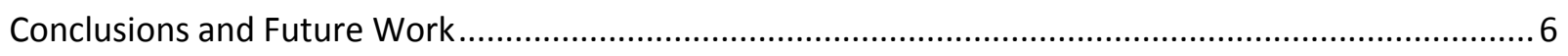

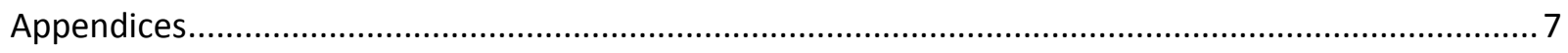

Appendix 1: Atomistic Studies of Defects in BCC Iron: Dislocations and Gas Bubbles ....................... 7

Appendix 2: Modeling the mechanical behavior and deformed microstructure of irradiation bcc materials using continuum crystal plasticity... 


\section{Introduction}

Understanding and improving microstructure stability in metals and alloys is central to the development of stable, high strength and high ductility materials for cladding and cores structures in advanced fast reactors. The research in this project focused on modeling plasticity at ambient and high temperatures caused by irradiation damage. The issues where we expect to make particular contributions are the mechanistic understanding and quantification of atomic-level processes of plastic deformation on long time scales and high temperatures that are relevant to structures in service in the cladding and core assembly of advanced reactors. The specific research objectives of this project are shown in Fig. 1. These were accomplished by the researchers with results archived in peer reviewed publications and presentations.

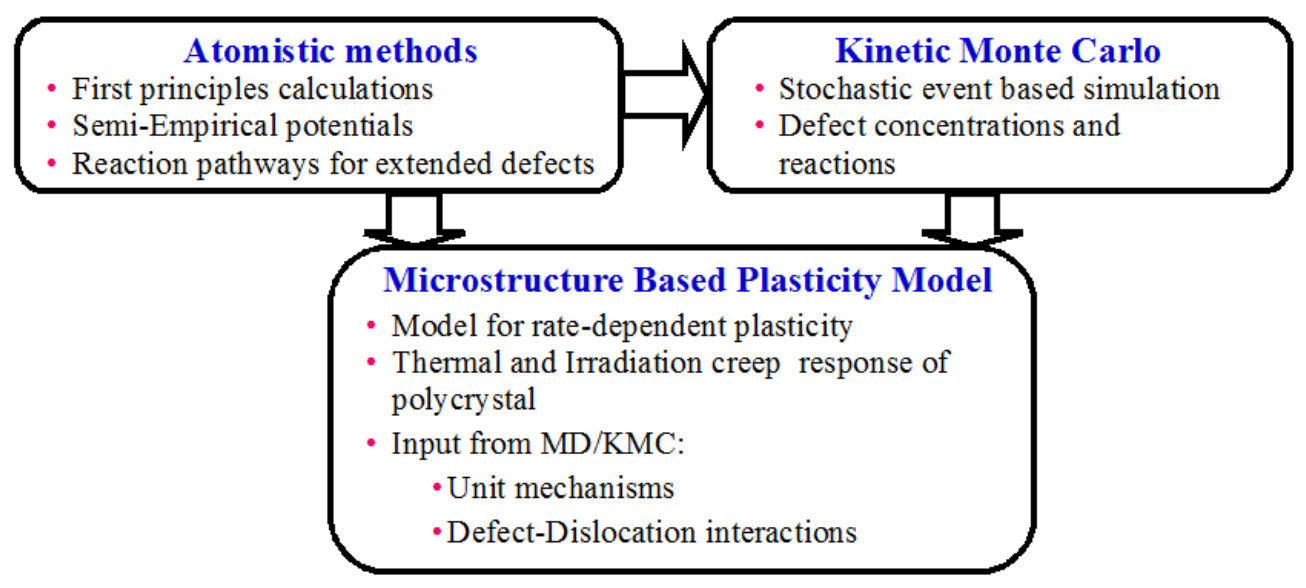

Fig. 1: Modules of research program in fundamental understanding of ambient and high temperature plasticity phenomena in structural materials in advanced reactors.

This project aimed to obtain a fundamental understanding of the unit processes driving plasticity at ambient and high temperatures under the influence of irradiation. One of the goals of the Advanced Fuel cycle Initiative (AFCl) program is to transmute minor actinides through irradiations in fuels to burnups of $20 \%$ or greater, which results in cladding doses of up to $200 \mathrm{dpa}$, and the need to develop radiation resistant materials. Irradiation changes the properties of cladding and duct materials as follows: increase in the ductile to brittle transition temperature; reduction of fracture toughness from low temperature irradiation (below $400 \circ$ C); irradiation creep, helium embrittlement (above $500 \mathrm{C}$ ) and swelling

Although these phenomena have been known for many years, the underlying fundamental mechanisms and their relation to the irradiation field have not been clearly demonstrated. Most models and theories of irradiation induced deformation and mechanical behavior rely on empirical parameters fit to experimental data. With improvements in computational techniques and algorithms, it is now possible to probe structure-property connections through the elucidation of fundamental atomic mechanisms. Often, these mechanisms involve defects of different dimensionality that exist and interact with each other to significantly affect material properties. 
For irradiated materials, point defects and clusters affect crystal plasticity. Dislocation-defect interactions may be associated with defects in the matrix, the modification of the local elastic constants due to the presence of defects and defect clusters and the effects of clusters and voids on the stacking fault energy. The formation of self interstitial loop rafts and the decoration of dislocations with self interstitial clusters have become important issues for understanding radiation hardening and embrittlement under cascade damage conditions. Dislocation motion is thought to be the main mechanism for deformation, because a fairly high density of network dislocations are generated during irradiation, and the dislocation sink strength for point defects is much higher that the grain boundary sink strength for point defects. Computational studies of dislocation activity can be performed at several different length and time scales that are shown in Fig. 2.

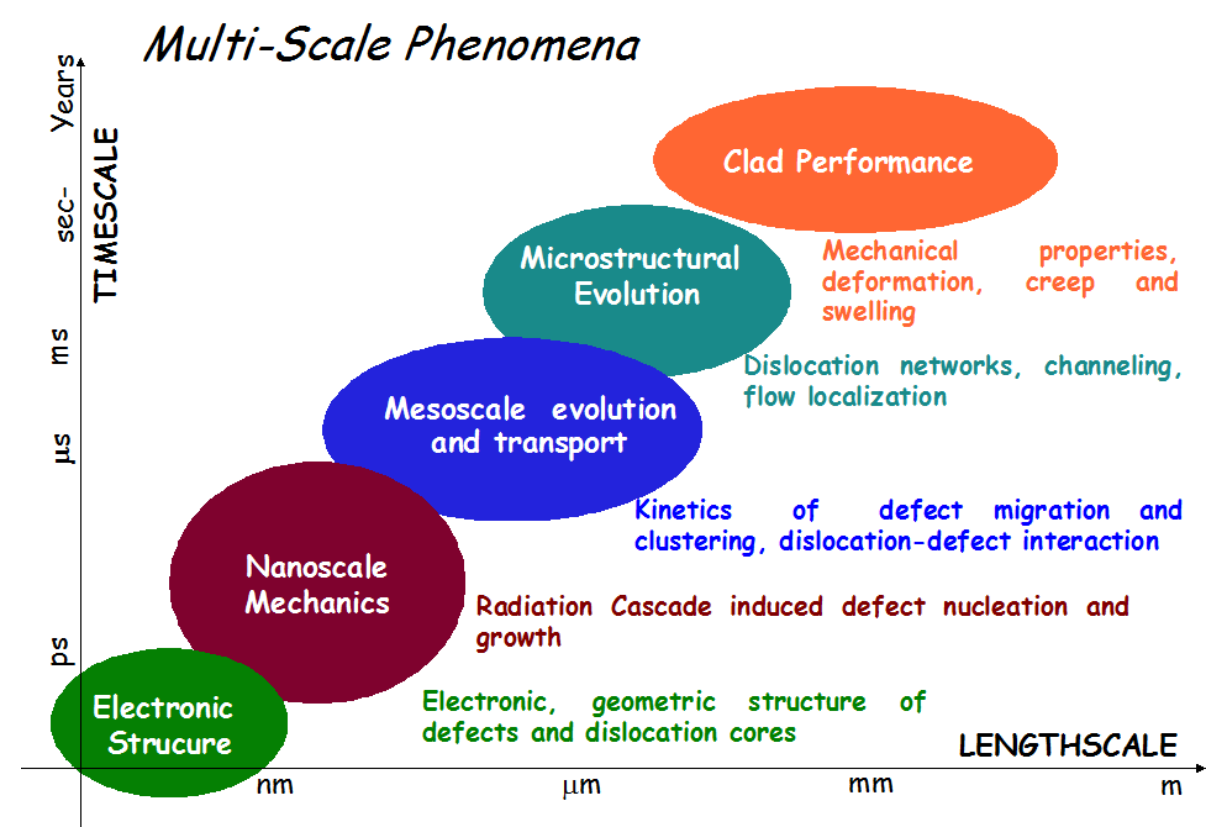

Fig. 2: Multiscale processes that govern dislocation activity and deformation processes.

Dislocations influence the diffusion tensor of defects and clusters; defects absorbed at dislocation lead to climb processes; diffusion processes are beyond the reach of conventional "brute force" MD; transition state theory and kinetic Monte Carlo models are used to compute reaction pathways and escape probabilities and saddle points and defect evolution; microstructure-sensitive plasticity models based on unit processes and mechanisms are much more predictive than those fit to empirical parameters

\section{Project Objective}

The overall goal of this three-year research program is to develop the methods and tools necessary to link unit processes analyzed using atomistic simulations involving interaction of vacancies and interstitials with dislocations, as well as dislocation mediation at sessile junctions and interfaces as affected by radiation, with cooperative influence on higher length scale behavior of polycrystals. 


\section{Work Performed and Results}

Work performed is described in the appendices and well documented in the publications and presentations listed below.

Appendix 1: Atomic Studies of Defects in BCC Iron: Dislocations and Gas Bubbles

Appendix 2: Modeling the mechanical behavior and deformed microstructure of irradiation bcc materials using continuum level crystal plasticity.

\section{Publications}

The following publications [1-14] resulted from the work done here. Only peer-reviewed journal publications are listed.

[1] Hayward E, Beeler B, Deo C. Multiple hydrogen trapping at monovacancies. Philosophical Magazine Letters 2012;92:217-25.

[2] Hayward E, Deo C. Synergistic effects in hydrogen-helium bubbles. Journal of Physics-Condensed Matter 2012;24.

[3] Hayward E, Deo C. ATOMIC SCALE MODELING OF HYDROGEN AND HELIUM IN BCC IRON. Fusion Science and Technology 2012;61:391-4.

[4] Hayward E, Deo C. Energetics of small hydrogen-vacancy clusters in bcc iron. Journal of PhysicsCondensed Matter 2011;23.

[5] Hayward E, Deo C. A Molecular Dynamics Study of Irradiation Induced Cascades in Iron Containing Hydrogen. Cmc-Computers Materials \& Continua 2010;16:101-16.

[6] Hayward E, Deo C, Uberuaga BP, Tome CN. The interaction of a screw dislocation with point defects in bcc iron. Philosophical Magazine 2012;92:2759-78.

[7] Patra A, McDowell DL. Continuum modeling of localized deformation in irradiated bcc materials. J Nucl Mater 2013;432:414-27.

[8] Patra A, McDowell DL. Crystal plasticity-based constitutive modelling of irradiated bcc structures. Philosophical Magazine 2012;92:861-87.

[9] Zhu T, Li J. Ultra-strength materials. Progress in Materials Science 2010;55:710-57.

[10] Peng C, Zhong Y, Lu Y, Narayanan S, Zhu T, Lou J. Strain rate dependent mechanical properties in single crystal nickel nanowires. Applied Physics Letters 2013;102. 
[11] Wang JW, Narayanan S, Huang JY, Zhang Z, Zhu T, Mao SX. Atomic-scale dynamic process of deformation-induced stacking fault tetrahedra in gold nanocrystals. Nature Communications 2013;4.

[12] Narayanan S, McDowell DL, Zhu T. Crystal Plasticity Model for BCC Iron Atomistically Informed by Kinetics of Correlated Kinkpair Nucleation on Screw Dislocations. Journal of the Mechanics and Physics in Solids 2013; (under review).

[13] Patra A, McDowell DL. A void nucleation and growth based damage framework to model failure initiation ahead of a sharp notch in irradiated bcc materials. Journal of Mechanics and Physics of Solids 2013; (under review).

[14] Patra A, Zhu T, McDowell DL. Constitutive equations for modeling non-Schmid effects in single crystal bcc-Fe at low and ambient temperatures. Acta Materialia 2013;submitted.

\section{Presentations}

The following presentations [1-20] were made by PIs and graduate students on work concerning this project.

[1] Deo C. Modeling Defect Mechanics in Materials for Nuclear Energy INVITED. International Conference on Computational and Experimental Engineering and Sciences Symposium on Nanostructured materials. Las Vegas, NV2010.

[2] Deo C. Atomistic Simulations of Intrinsic and Extrinsic Defects in bcc iron INVITED. Innovative Materials Immune to Irradiation, Department of Energy-Nuclear Energy Co-Sponsored Workshop Vail, CO2010.

[3] Hayward E, Deo C. Finding Minimum Energy States of Hydrogen-Vacancy Clusters in Iron. American Nuclear Society Annual Meeting. Hollywood, FL2011.

[4] Hayward E, Deo C. The Thermodynamics of Hydrogen-Helium-Vacancy Clusters in BCC Iron. 25th Symposium on Effects of Radiation on Nuclear Materials, ASTM International. Anaheim, CA2011.

[5] Hayward E, Deo C. The Energetics of Hydrogen-Vacancy Clusters in BCC Iron Materials Research Society, Symposium on Fundamental Science of Defects and Microstructure in Advanced Materials for Energy. San Francisco, CA2011.

[6] Zhu T. Workshop on Nanomaterials at the Integrated Nanotechnologies (CINT), Sandia National Lab, Albuquerque, New Mexico. 2012.

[7] Zhu T. International Workshop on Bulk Nanostructured Metals, Kyoto, Japan. 2012.

[8] Zhu T. Oberwolfach Workshop on Mechanics of Materials, Oberwolfach, Germany. 2011.

[9] Zhu T. 2nd International Workshop on Nanoplasticity, Lake Bostal, Germany. 2011. 
[10] Zhu T. Computational Chemistry and Materials Science Summer Institute, Lawrence Livermore National Laboratories, Livermore, CA. 2011.

[11] Zhu T. 4th International workshop on materials behavior at micro- and nano-scale, Xi'an, China. 2011.

[12] Zhu T. International Symposium on Multiscale Modeling and Simulation of Materials, Shenyang, China. 2010.

[13] Zhu T. 16th US National Congress of Theoretical and Applied Mechanics, State College, PA. 2010.

[14] Zhu T. 2nd NSF workshop on Multiscale Dislocation Dynamics, San Diego, CA. 2010.

[15] Zhu T. Materials Research Society Fall meeting, Boston, MA, November 2009. 2010.

[16] Patra A, McDowell DL. Crystal Plasticity Modeling of Radiation Damage in BCC Structural Materials. ANS Annual Meeting. Hollywood, FL2011.

[17] Patra A, McDowell DL. Modeling Localized Deformation in Irradiated BCC Metals using Continuum Crystal Plasticity. 49th Annual Technical Meeting of the Society of Engineering Science. Atlanta, GA2012.

[18] Patra A, McDowell DL. Crystal Plasticity Modeling of Localized Deformation in Irradiated BCC Metals. MRS Fall Meeting. Boston, MA2012.

[19] Patra A, McDowell DL. Mesoscopic Modeling of Dislocation-Defect Interactions and Flow Localization in Irradiated bcc Metals. TMS 2013 Annual Meeting and Exhibition. San Antonio, TX2013.

[20] Patra A, McDowell DL. Constitutive Equations for Dislocation Core Spreading in BCC-Fe Accounting for Dislocation-Dislocation Interactions and Finite Temperature Effects. MS\&T'13. Montreal, Quebec, Canada2013.

\section{Conclusions and Future Work}

The question of radiation damage and consequent plasticity evolution is important. Here we have made progress in linking together different simulation methods and in developing understanding of plasticity as a result of radiation damage. Also we have started investigating the effect of hydrogen on nuclear structures. Future work could proceed along similar lines and probe unit mechanisms. Also of interest would be the uncertainty linkages between various scales of operation and the propagation of uncertainty as the methods vary and information is passed. 


\section{Appendices}

Appendix 1: Atomistic Studies of Defects in BCC Iron: Dislocations and Gas Bubbles This appendix is attached next and integrated in the single pdf

Appendix 2: Modeling the mechanical behavior and deformed microstructure of irradiation bcc materials using continuum crystal plasticity

This appendix is attached last and integrated in the single pdf 


\section{APPENDIX 1}

ATOMISTIC STUDIES OF DEFECTS IN BCC IRON: DISLOCATIONS

\section{AND GAS BUBBLES}




\section{SUMMARY}

Appendix 1: Atomistic Studies of Defects in BCC Iron: Dislocations and Gas Bubbles contains content from 5 refereed publications.

The structure and interactions of the defects in material on an atomistic scale ultimately determine the macroscopic behavior of that material. A fundamental understanding of how defects behave is essential for predicting materials failure; this is especially true in an irradiated environment, where defects are created at higher than average rates. In this work, we present two different atomistic scale computational studies of defects in body centered cubic (bcc) iron. First, the interaction energies between screw dislocations (line defects) and various kinds of point defects will be calculated, using anisotropic linear elastic theory and atomistic simulation, and compared. Second, the energetics and behavior of hydrogen and hydrogen-helium gas bubbles will be investigated. 


\section{TABLE OF CONTENTS}

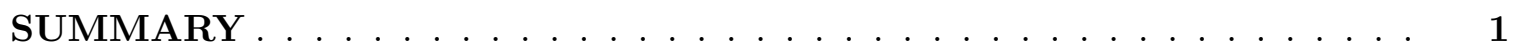

LIST OF TABLES $\ldots \ldots \ldots \ldots \ldots \ldots \ldots \ldots \ldots \ldots$

LIST OF FIGURES $\ldots \ldots \ldots \ldots \ldots \ldots \ldots \ldots \ldots \ldots$

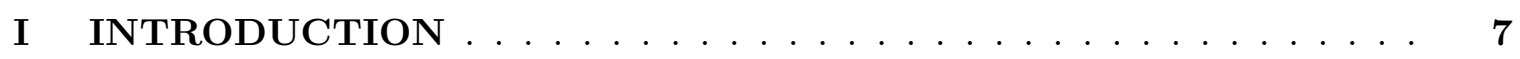

1.1 Radiation Damage $\ldots \ldots \ldots \ldots \ldots \ldots \ldots$

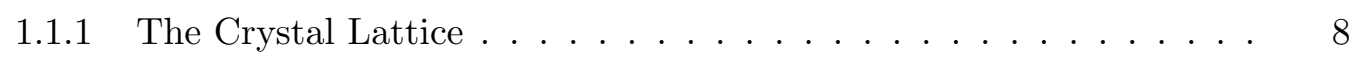

1.1 .2 Dislocations . . . . . . . . . . . . . . . . 9

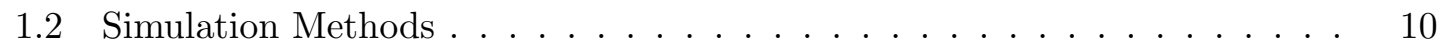

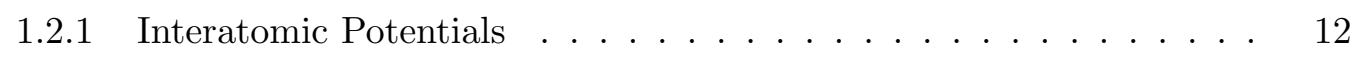

II SCREW DISLOCATION-DEFECT INTERACTIONS . . . . . . . 19

2.1 Motivation and Literature Review . . . . . . . . . . . . . 19

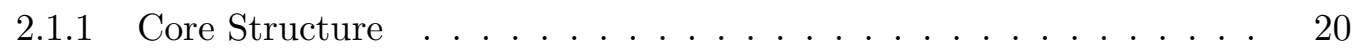

2.1.2 Dislocation-Defect Interaction . . . . . . . . . . . . . . 21

2.2 Linear Elasticity Theory _ . . . . . . . . . . . . . . . . . . . 22

2.2 .1 Dipole Tensor . . . . . . . . . . . . . . . . . . . . . . 23

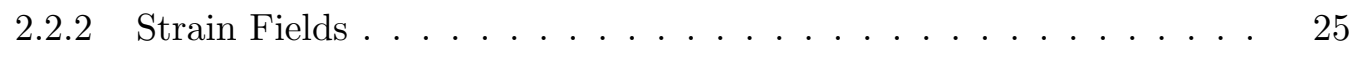

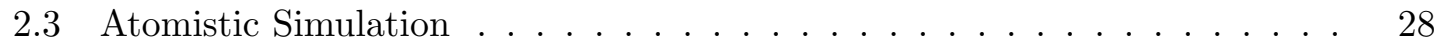

2.4 Results . . . . . . . . . . . . . . . . . . . . 29

$2.4 .1<110>$ Dumbbell . . . . . . . . . . . . . . 30

$2.4 .2<111>$ Dumbbell . . . . . . . . . . . . . . . . 33

$2.4 .3<100>$ Dumbbell ................... . . 37

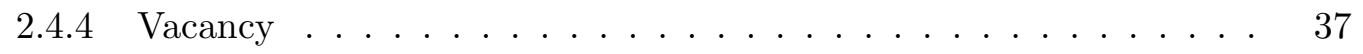

2.4.5 Absorption by the Core . . . . . . . . . . . . . . 40

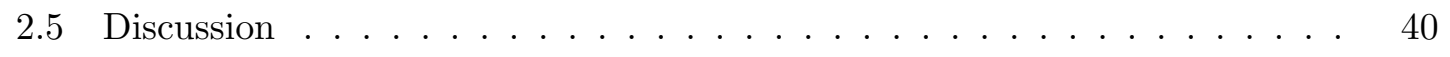

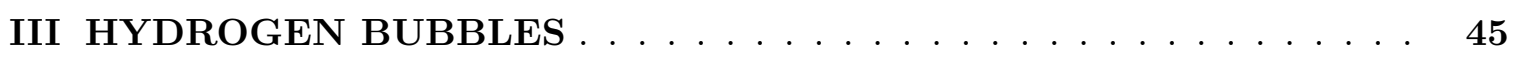

3.1 Motivation and Literature Review . . . . . . . . . . . . . 45

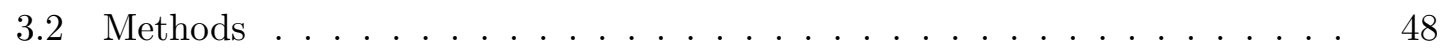

3.3 Results . . . . . . . . . . . . . . . . . . 52 
3.3 .1 Performance of Interatomic Potentials _ . . . . . . . . . . . 52

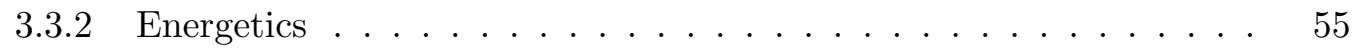

3.3.3 Multiple Hydrogen Occupancy of Monovacancies . . . . . . . . . 59

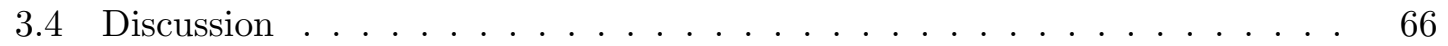

IV HYDROGEN - HELIUM BUBBLES . . . . . . . . . . . . . 70

4.1 Motivation and Literature Review . . . . . . . . . . . . . . . . . . 70

4.2 Methods . . . . . . . . . . . . . . . . . . . . 71

4.3 Results . . . . . . . . . . . . . . . . . . 72

4.3 .1 Bubble Structure . . . . . . . . . . . . . . . . 73

4.3 .2 Interaction between $\mathrm{H}$ and $\mathrm{He} \ldots \ldots \ldots 77$

4.3 .3 Binding of Vacancies and Interstitials . . . . . . . . . . . . 80

4.3 .4 Loop Punching . . . . . . . . . . . . . . . 80

4.4 Discussion . . . . . . . . . . . . . . . . . . 83

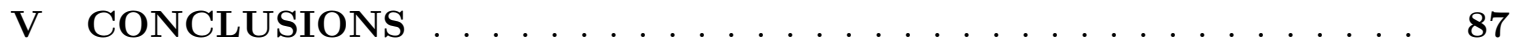

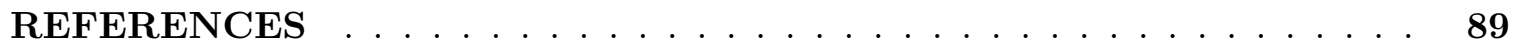




\section{LIST OF TABLES}

1 Parameters for $\mathrm{H}-\mathrm{He}$ interatomic potential . . . . . . . . . . . . . 18

2 The components of the dipole tensor $\mathbf{P} \ldots \ldots \ldots \ldots 24$

3 Total and formation energies of multiple hydrogen atoms at a monovacancy 64

4 Binding energies of multiple hydrogen atoms at a monovacancy . . . . . . 65 


\section{LIST OF FIGURES}

1 Four snapshots from a $10 \mathrm{keV}$ cascade in pure $\mathrm{Fe} \ldots \ldots . \ldots$

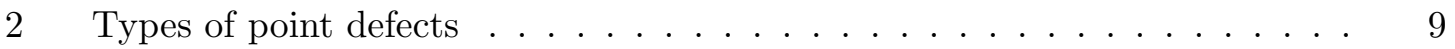

3 Schematics of edge and screw dislocations . . . . . . . . . . . . 10

4 Summary of multi-scale modeling . . . . . . . . . . . . . . . . 11

5 The interatomic potential well for $\mathrm{H}-\mathrm{He} \ldots \ldots \ldots \ldots$

6 Differential displacement map of screw core . . . . . . . . . . . . 21

$7 \quad$ Schematic for calculating the dipole tensor . . . . . . . . . . . . 25

8 The coordinate system for the screw dislocation . . . . . . . . . . . 28

9 Interaction energy of the $\left[\begin{array}{lll}1 & \overline{1} & 0\end{array}\right]$ dumbbell $\ldots \ldots \ldots \ldots$. . . . . . . . . . 32

10 Error of the interaction energy of the $\left[\begin{array}{lll}1 & \overline{1} & 0\end{array}\right]$ dumbbell $\ldots \ldots \ldots$. . . . . . 33

11 Interaction energy of the $\left[\begin{array}{lll}1 & 1 & 0\end{array}\right]$ dumbbell $\ldots \ldots \ldots \ldots$. . . . . . . . 34

12 Final orientations of the $\left[\begin{array}{lll}1 & 1 & 1\end{array}\right]$ dumbbell $\ldots \ldots \ldots \ldots$

13 Atomistic interaction energy of the $\left[\begin{array}{lll}1 & 1 & 1\end{array}\right]$ dumbbell $\ldots \ldots \ldots$. . . . . . . 36

14 Theoretical interaction energy of the $\left[\begin{array}{lll}1 & 1 & 1\end{array}\right]$ dumbbell $\ldots \ldots \ldots$. . . . . . 36

15 Interaction energy of the $\left[\begin{array}{lll}1 & 0 & 0\end{array}\right]$ dumbbell $\ldots \ldots \ldots \ldots$

16 Final orientations of the $\left[\begin{array}{lll}1 & 0 & 0\end{array}\right]$ dumbbell $\ldots \ldots \ldots \ldots$

17 Interaction energy of the vacancy . . . . . . . . . . . . . . 39

18 Core structure after absorption of dumbbell . . . . . . . . . . . . . . . 41

19 Core structure after absorption of vacancy . . . . . . . . . . . . . . 41

20 Schematic of the preferred defect positions . . . . . . . . . . . 42

21 Schematic of the energy minimization of a gas bubble $\ldots \ldots \ldots \ldots$

22 Pair potential for $\mathrm{Fe}-\mathrm{H}$ and $\mathrm{H}-\mathrm{H}$ interactions . . . . . . . . . . . 54

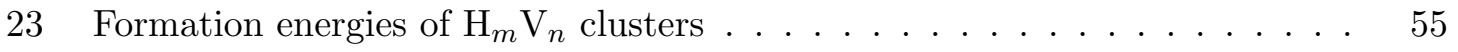

24 Binding energies of vacancies to $\mathrm{H}_{m} \mathrm{~V}_{n}$ clusters $\ldots \ldots \ldots \ldots$

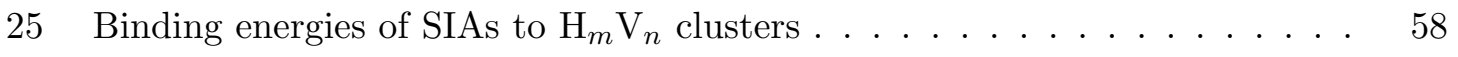

26 Binding energies of hydrogen atoms to $\mathrm{H}_{m} \mathrm{~V}_{n}$ clusters $\ldots \ldots \ldots \ldots$

27 Generally accepted sites for six hydrogen atoms around a monovacancy . . 60

28 The positions of one, two, and three hydrogen atoms around a monovacancy

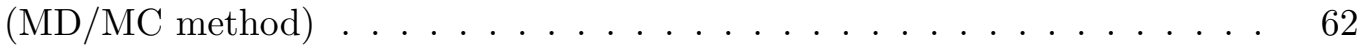


29 The positions of four hydrogen atoms around a monovacancy . . . . . . . 62

30 The positions of five hydrogen atoms around a monovacancy $\ldots \ldots$. . . . 63

31 The positions of six hydrogen atoms around a monovacancy . . . . . . . 63

32 The binding energy of the $m^{\text {th }}$ hydrogen atom to a $\mathrm{H}_{m-1} \mathrm{~V}$ cluster $\ldots \ldots 6$

33 The low energy configurations of three hydrogen-helium-vacancy clusters . 73

34 Structure of the unrelaxed configuration of a cluster of 6 vacancies . . . . . 74

35 The radii in Angstroms of a $\mathrm{H}_{m} \mathrm{He}_{j} \mathrm{~V}_{6}$ cluster as $m$ is increased . . . . . 75

36 The radii in Angstroms of a $\mathrm{H}_{m} \mathrm{He}_{j} \mathrm{~V}_{6}$ cluster as $j$ is increased $\ldots \ldots$. . . 76

37 The binding energies of hydrogen and helium to $\mathrm{H}_{m} \mathrm{He}_{j} \mathrm{~V}_{6}$ clusters $\ldots . . .79$

38 Binding energies of helium atoms, SIAs, and vacancies to $\mathrm{H}_{m} \mathrm{He}_{j} \mathrm{~V}_{6}$ clusters 81

39 Curves for Frenkel pair formation energy for pure helium bubbles . . . . . . 82

40 Curves showing the difference in energy between clusters with and without a Frenkel pair . . . . . . . . . . . . . . . . . . 84

41 The minima of the energy difference curves for clusters with and without Frenkel pairs .......................... 85 


\section{CHAPTER I}

\section{INTRODUCTION}

The interactions between defects within a material's microstructure determine the ultimate behavior of the material on a macroscopic scale. Dramatic macroscopic behavioral changes include creep, plasticity, radiation-induced segregation, embrittlement, changes in brittle to ductile transition temperature, hardening, and cracking. Atomistic studies provide a valuable tool for investigating these interactions in a detailed and fundamental way.

Ferritic steels are a popular choice for current and future reactor designs. These steels are mainly composed of body centered cubic, or $\alpha$-phase iron. Thus, this material is a relevant and important choice for the matrix in which we perform defect studies.

The goal of this work is to examine the interactions and energetics of defects on the microscale, in order to gain a more fundamental understanding of their behavior. We use atomistics as a tool for this purpose.

\subsection{Radiation Damage}

A material undergoing irradiation experiences a high rate of defect creation due to a high volume of incident particles, so the study of defect behavior is particularly important to the study of irradiated materials. The process of radiation damage proceeds in several stages, as is shown in Figure 1. First, and incident particle transfers some or all of its energy to an atom in the crystal, resulting in the atom being removed from its lattice site. This atom, known as the primary knock-on atom, or PKA, goes on to interact with other atoms in the system, creating a cascade of damage. Ultimately, some of the affected atoms will come

to rest in off lattice sites as interstitials, leaving behind an empty lattice site known as a vacancy. A vacancy and an interstitial are together known as as Frenkel pair. The majority of Frenkel pairs created during a damage cascade will recombine within several picoseconds. However, a small number of the created defects some will remain, usually creating a structure consisting of a core of vacancies surrounded by a shell of interstitials. Over time, these 


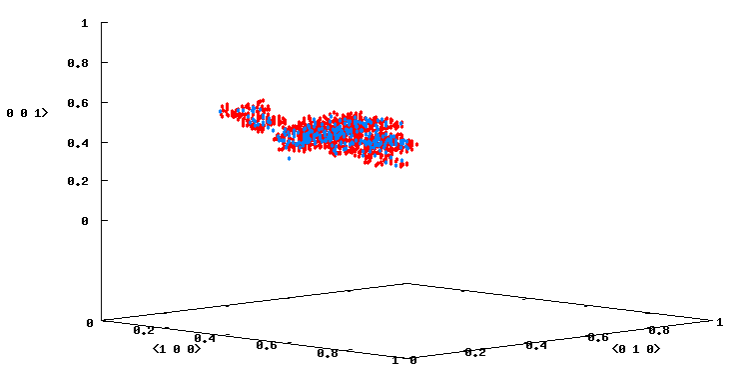

(a) $0.1 \mathrm{ps}$

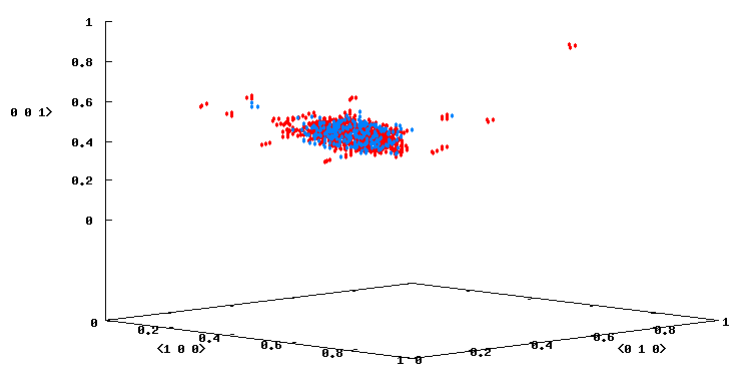

(c) 4.0 ps - partially relaxed

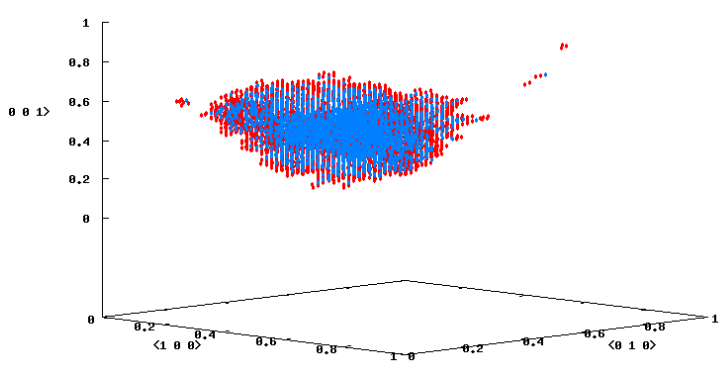

(b) 0.9 ps - near peak damage

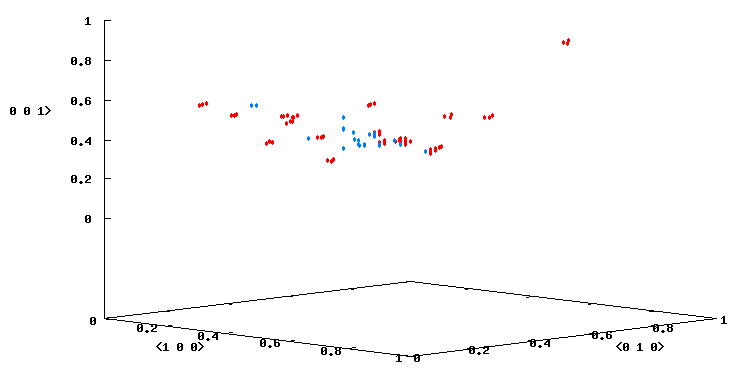

(d) 20.0 ps - fully relaxed

Figure 1: Four snapshots from a $10 \mathrm{keV}$ cascade in pure Fe. Red dots show interstitials, blue dots show vacancies. Note the vacancy rich core surrounded by interstitials.

point defects migrate through the lattice, cluster together, and form extended defects, such as dislocation lines and loops.

\subsubsection{The Crystal Lattice}

The Miller indices [86] are the most commonly used notation system for describing planes and directions in crystal lattices. A unit cell coordinate system is defined by three lattice vectors (for a cubic lattice, these will be orthogonal). The inverse intercepts along these

lattice vectors expressed in integer form define a plane $(h k l)$, a family of planes $\{h k l\}$, a direction $[h k l]$, or a family of directions $\langle h k l\rangle$, of the unit cell. Thus, if an index is zero, the plane does not intersect the corresponding axis. By convention, a negative integer is written with a bar over its value (e.g. $\overline{1})$.

This notation is particularly useful for for describing orientations of point defects in materials. Generally, an interstitial will share a lattice site with another matrix atom; this is known as an interstitial dumbbell. The orientation of the dumbbell can be described using the Miller indices; several low-index orientations are shown in Figure 2. 


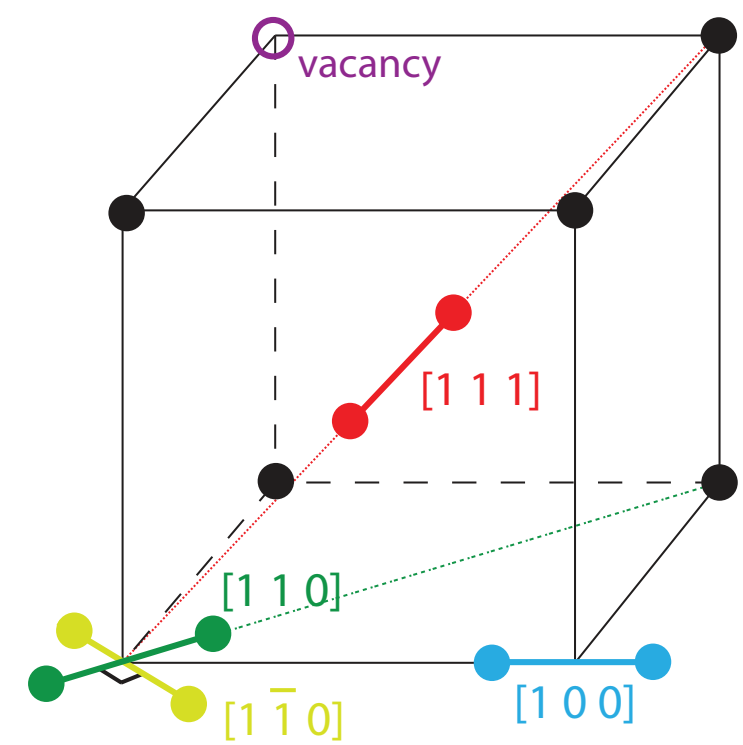

Figure 2: A body-centered cubic (bcc) cell with various orientations and types of point defects.

In the body-centered cubic (bcc) cell, there a matrix atom on every corner of a cube, plus one atom in the center of the cell. In iron, this structure is referred to as $\alpha$-iron or ferritic iron. This is the structure that is stable below $1670{ }^{\circ} \mathrm{F}$. In $\alpha$-iron, the $<110>$ dumbbell is the most stable (lowest energy) interstitial orientation; this is in contrast to other bcc transition metals, in which the $<111>$ orientation is preferred.

\subsubsection{Dislocations}

Dislocations are topological irregularities in the crystal structure. There are two main types, edge and screw; additionally, a dislocation can be of mixed character. An edge dislocation can be pictured as a block into which an extra half-plane of atoms has been inserted. A screw dislocation can be generated by making a cut into a block of material, and slipping the atoms on either side relative to each other. The result is a helical structure about the dislocation line (the terminus of the cut in the bulk material). These two structures are illustrated schematically in Figure 3. Dislocations may be of line form, with the ends terminating at grain boundaries or surfaces, or they may be loops, in which they are entirely enclosed within the crystal.

If in a perfect crystal, a closed rectangle in a plane can be drawn about the site of 

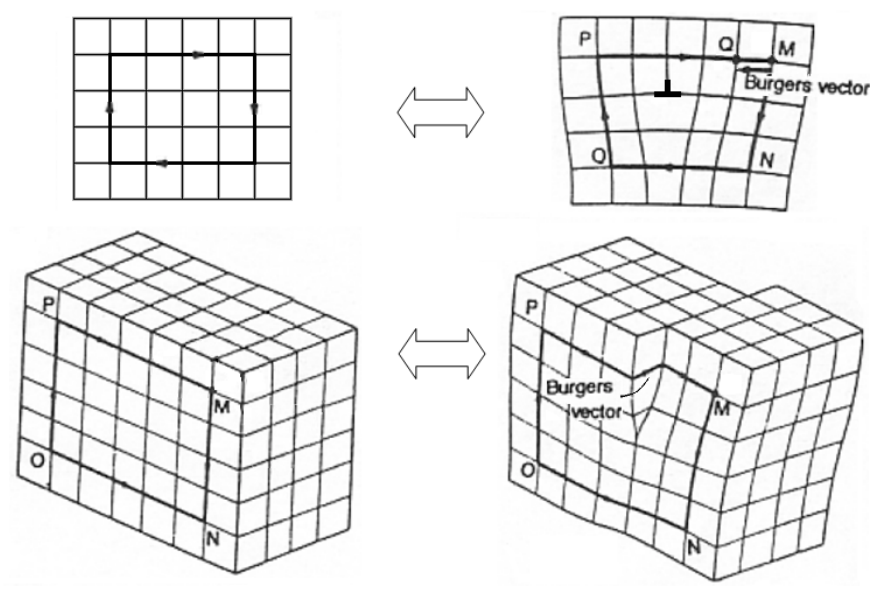

Figure 3: Schematics of the two types of dislocations; edge is on top, screw is on the bottom. Image from Wikimedia Commons via the Creative Commons Attribution-Share Alike 3.0 Unported license.

the dislocation, when the dislocation is present, an attempt to draw the same rectangle will result in a gap in the circuit. The vector required to close this gap is known as the Burgers vector $\mathbf{b}$. The relationship between the Burgers vector $\mathbf{b}$ and the dislocation line $\xi$ characterize the type of dislocation - in an edge dislocation, $\mathbf{b}$ is perpendicular to $\xi$, while in a screw dislocation, the two are parallel.

Dislocations strongly affect macroscopic properties. The presence of dislocations lowers the shear stress needed to deform a material, since dislocations may move incrementally through climb and glide. The interactions with other defects affect these processes; for example, some defects may act as pinning sites, while others may be absorbed to aid movement.

\subsection{Simulation Methods}

The modeling of materials is an inherently multi-scale problem, both in time and length scales (see Figure 4). Smaller scale methods are more accurate because they can capture more of the relevant interactions between atoms. However, they are limited in applicability because of this same feature - the number of atoms goes up, the calculation requires significantly more computational resources to detail all of the interactions. Generally, highly accurate small scale calculations can be used to generate input parameters for larger scale simulations. These larger scale simulations are able to more directly connect with observed 


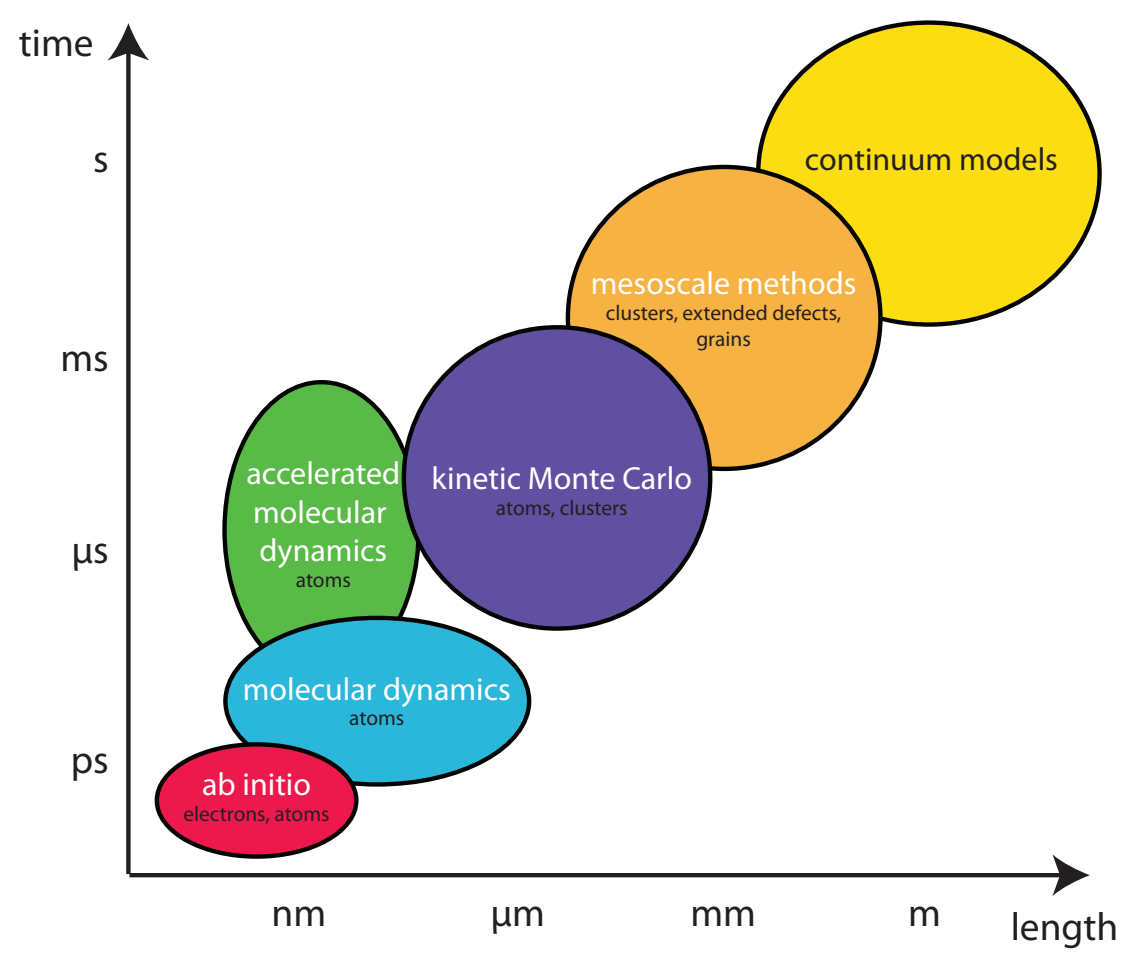

Figure 4: Modeling of materials is an inherently multiscale task. This graph shows the approximate time and length scales over which a particular method is applicable.

phenomena and can (hopefully) be used in predictive capacity. Simulation methods can be categorized by what type of entity is taken as the basic unit for interactions. That is, electronic structure calculations consider individual electrons and atoms, while large scale models may consider entire grains as their basic unit.

First principles, or $a b$ initio, methods seek to find a solution to the Schrödinger equation for a group of atoms and to determine the electronic structure. There are a wide variety of methods available to quantum chemists, they vast majority of which rely on the BornOppenheimer approximation. This approximation assumes that the motions of the electrons and the nuclei are essentially separable, since the atomic nuclei are much heavier than the electrons. Thus, the ground state of the electrons can be solved based on fixed nuclear positions. Then, the energy of the system for displaced nuclei can be determined.

Density functional theory (DFT) is one very popular ab initio method, in which the ground state energy of a group of atoms is a unique functional of the electron density. Minimizing this functional theoretically results in the ground state energy that would be found 
by solving the Schrödinger equation. However, finding the appropriate density functional is the challenge of DFT. In practice, this problem is solved for iteratively.

Although ab initio methods are extremely valuable, since they consider the most fundamental details of a problem, the most advanced methods in this category are currently limited to several hundred atoms. Molecular dynamics methods are the next step up in time and length scale and can solve for millions of atoms for times of up to microseconds, however they do not solve for electrons directly.

Classical molecular dynamics codes operate on a fairly simple principle. For all the atoms in a system, Newton's equations of motion are solved for known atomic masses and velocities to determine trajectories. The interactions between atoms are described via interatomic potentials, which will be described in more detail in Section 1.2.1. Molecular dynamics is the chosen method for the majority of this work.

Most molecular dynamics calculations have the capacity to model millions of atoms, generally for nanosecond timescales. However, an additional class of methods, known as accelerated molecular dynamics are able to look at even longer timescales. This is particularly useful when the processes one wishes to model are uncommon or infrequent events (e.g. vacancy diffusion). An example of this class of codes is temperature accelerated dynamics $[132]$.

Kinetic Monte Carlo methods may use individual atoms or clusters of atoms as their basic units. These methods rely on probabilities of events occurring to determine the longtime behavior of a system. These probabilities are often based on binding and diffusion energies discovered by the smaller scale methods. At even longer time and length scales, mesoscopic and continuum models follow the behavior of extended defects and grains to develop constitutive models, based on data found in smaller scale simulations.

\subsubsection{Interatomic Potentials}

Interactions between particles in atomistic simulations are described by functions and parameters known as interatomic potentials. Choosing an appropriate potential is essential for obtaining quality results in any atomistic calculation. 
The simplest types of interatomic potentials are pair potentials. Pair potentials are computationally very inexpensive and are often relatively easy to compute. This class of potentials assumes that by summing up all the individual pair bonds the total energy of the solid $E_{c o h}$ can be recovered. However, this assumption turns out to be impossible to prove theoretically.

An often used form is the Lennard-Jones (L-J) potential [74], where the potential energy $V$ between particles $i$ and $j$ at a distance $r$ is given by

$$
V\left(r_{i j}\right)=4 \varepsilon\left[\left(\frac{\sigma}{r}\right)^{12}-\left(\frac{\sigma}{r}\right)^{6}\right]
$$

Here, $\varepsilon$ is the depth of the potential well and $\sigma$ is the distance at which the potential becomes zero. The second term is responsible for the attractive part of the potential, representing the weak van der Waals bond. The first term models strong repulsion at short distances due to electronic overlap; the exponent is given a value of 12 purely for ease of computation, but has no theoretical justification. Similar to other pair potential forms, the L-J potential is inadequate for modeling strongly bonded systems or metals.

At close distances, the repulsion between two nuclei is essentially Coulombic, while at larger distances, the electron clouds of the atoms screen the nuclei. This may be written as

$$
V\left(r_{i j}\right)=\frac{Z_{1} Z_{2} e^{2}}{r} \varphi\left(\frac{r}{a}\right)
$$

where $Z_{1}$ and $Z_{2}$ are the atomic numbers of the two interacting nuclei, $e$ is the charge on an electron, and $\varphi$ is the screening potential. A commonly used version of this form is the Ziegler-Biersack-Littmark (ZBL) repulsive potential, in which the universal screening function is given by

$$
\varphi(x)=0.1818 e^{-3.2 x}+0.5099 e^{-0.9423 x}+0.2802 e^{-0.4029 x}+0.02817 e^{-0.2016 x},
$$

and

$$
a=\frac{0.8854 a_{B}}{Z_{1}^{0.23}+Z_{2}^{0.23}}
$$

where $a_{B}=0.529 \AA$ is the Bohr radius. This potential is purely repulsive, but theoretically correct at small distances. 
Thus, it is a common practice to create an overall interatomic pair potential for interacting atoms by connecting different types of pair potentials at different distances with polynomial splines. In this way, a potential can be generated which has both long and short range attraction and repulsion that is theoretically correct.

In reality, a many-body potential is needed to account for the influence that bonds between atoms have on each other. To deal with this, the embedded-atom method (EAM) was developed by Daw and Baskes in 1983 [25, 26, 27] for metallic solids. Essentially, each atom is treated as if it were a defect embedded in an electron gas created by the presence of all the other atoms. The cohesive energy of the system can be described in two parts by an embedding energy and by an electrostatic interaction with a sum over all atoms in the system:

$$
E_{c o h}=\sum_{i} G_{i}\left(\sum_{j \neq i} \rho_{j}^{a}\left(R_{i j}\right)\right)+\frac{1}{2} \sum_{i, j(j \neq i)} \phi_{i j}\left(R_{i j}\right)
$$

where $G$ is the embedding energy, $\rho^{a}$ is the spherically averaged atomic electron density, $R_{i j}$ is the distance between atoms $i$ and $j$, and $\phi$ is an electrostatic, two-atom interaction. With this formulation, atoms that are near defects or surfaces, for example, will feel a different density profile than atoms in the bulk, allowing the potential to treat significantly more complex systems than pair potentials alone.

While more complex systems can be treated, implementing the method is no more difficult than implementing a pair potential. The functions $G(\rho)$ and $\phi(R)$ can be found either from first principles or using semi-empirical methods. Normally, they are fit to data from a particular (pure) metal, such as lattice constants, elastic constants, or defect formation energies.

A similar method was developed simultaneously by Finnis and Sinclair (FS) [36]. For pure metals, the two methods are exactly equivalent. For alloys, the FS ansatz requires different functionals $\rho$ for interactions between different elements, while EAM uses an averaged one.

To simulate all of the interactions between the three different elements considered in this work, at least six potentials are required. In practice, even more interatomic potentials 
were used. The relative merits and properties of all potentials used or considered in this work are discussed in this section.

\subsubsection{1 $\mathrm{Fe}-\mathrm{Fe}$}

The iron-iron interactions in our simulations are described by Ackland's potential [3]. This EAM style potential is a slightly improved form of a potential originally published by Mendelev [83]; we refer to potential \#2 of that paper. The original potential is intended to provide an accurate description of both crystalline and liquid iron; thus, it is sensitive to a wide-range of separation distances between atoms. The potential is fit to perfect crystal properties, as well as to forces found from first principles calculations. The new parametrization takes advantage of further ab initio calculations performed at small interatomic distances with the goal of accurately describing point defect properties. This makes the potential ideal for describing radiation damage events and interactions between defects.

The Ackland potential for Fe-Fe is widely used in radiation damage studies, as it is provides an excellent description of defect behavior. Of course, it is not the only available potential for iron available [95, 93, 2, 31]; even Mendelev et al. provides alternate parameterizations of the above potential which may perform slightly better in particular situations [83]. The potential we choose does not take into account magnetic effects of iron, which may be important in some situations; there are potentials available with the capability [31], however they are currently less suitable for describing defect interactions appropriately than is the potential of Ackland et al.

\subsubsection{2 $\mathrm{Fe}-\mathrm{H}$ and $H-H$}

We take both the iron-hydrogen and hydrogen-hydrogen interactions from Ramasubramaniam et al. [119, 121]. Ramasubramaniam provides two parameterizations of the potential, called A and B. The first takes it's iron-iron interactions from Mendelev's potential \#4 [83], while the latter describes Fe-Fe via the improved Ackland potential [3] based on Mendelev's potential \#2 [83], the potential we use for Fe-Fe interactions. Thus, potential B is a natural choice for our calculations. Additionally, the authors provide a slightly different version 
of potential B, called $\mathrm{B}^{\prime}$, which is available for download from their website ${ }^{1}$, but is not described in detail in the literature. We test both potential B and potential $\mathrm{B}^{\prime}$, as described in Chapter 3. Potential B is fit to bulk, surface, and vacancy DFT data, while $\mathrm{B}^{\prime}$ is fit only to bulk and vacancy data, but has better performance regarding strain fields in the bulk.

\subsubsection{3 $\mathrm{Fe}-\mathrm{He}$}

There are many interatomic potentials for the iron-helium system, many of which have come out just in the past several years. These include the "classic" potential of Wilson [155], a three-body potential from Seletskaia et al. [125, 135], and a pair potential from Gao et al. $[41]$.

For this work, we choose to use the purely repulsive pair potential of Juslin and Nordlund [69]. This potential is ideally parameterized to be used along with Ackland's potential for Fe-Fe [3]. For small interatomic distances, calculations of an Fe-He dimer from the DMol97 program package are used. Farther out, the potential is fit to DFT data. The authors caution that potential is not appropriate for molecules, but only for helium within the iron matrix.

\subsubsection{4 $\mathrm{He}-\mathrm{He}$}

Helium is an extremely stable, almost completely inert element. Beck [6] developed a potential for helium-helium interactions given by

$$
V\left(r_{i j}\right)=A \exp \left(-\alpha r-\beta r^{6}\right)-\frac{0.869}{\left(r_{i j}^{2}+b^{2}\right)^{3}}\left(1+\frac{2.709+3 b^{2}}{r_{i j}^{2}+b^{2}}\right),
$$

where $b=0.675 \AA, \alpha=4.390 \AA^{-1}, \beta=3.746 \times 10^{-4} \AA^{-6}$, and $A=398.7 \mathrm{eV}$.

Beck fit this potential so that the long-range attraction is theoretically correct, and the short-range repulsion agrees with both experimental and calculated results. Additionally, the depth and shape of the well were determined by a fit to the second virial coefficient, which provides a correction to the ideal gas law for the pressure of a multi-particle system. The potential has its minimum of $V(r)=8.936 \times 10^{-4} \mathrm{eV}$ at $r=2.969 \AA$.

\footnotetext{
${ }^{1}$ http://www.princeton.edu/mae/people/faculty/carter/homepage/research/potentials/
} 
This potential has been used extensively by researchers within the field of nuclear materials; thus, comparison of our results with those of other researchers is straightforward.

\subsubsection{5 $\mathrm{H}-\mathrm{He}$}

We present a new interatomic potential which is suitable for simulating the interactions between hydrogen and helium.

Hydrogen and helium have an extremely weak van der Waals attraction, making this system a good candidate for a L-J potential. Exact quantum mechanical calculations on the system were performed by Bhattacharya and Anderson [9] using a Green's-function Monte Carlo method. They found the H-He potential energy for completely separated atoms as well as for eleven internuclear distances. They compared their results to multiple experimental and $a b$ initio studies, with good agreement.

We have fit a Lennard-Jones potential to these data points, finding values of $\varepsilon=0.0006$ $\mathrm{eV}$ and $\sigma=3.0862 \AA$. This gives a maximum well depth of $0.0006 \mathrm{eV}(6.96 \mathrm{~K})$ at an interatomic distance of $3.464 \AA$ (6.55 bohr).

In order to simulate the correct short distance repulsion between hydrogen and helium, we connect the Lennard-Jones form to a ZBL form at short distances. An inverse polynomial form is chosen for the spline, and parameters are chosen to guarantee continuity of the potential and its first derivative. The potential energy between a hydrogen atom and a helium atom at distance $r$ is given by

$$
V\left(r_{i j}\right)= \begin{cases}\frac{Z_{1} Z_{2} e^{2}}{r} \varphi\left(\frac{r}{a}\right), & \text { for } r<r_{1} \\ D_{0}+\frac{D_{1}}{r}+\frac{D_{2}}{r^{2}}+\frac{D_{3}}{r^{3}}, & \text { for } r_{1} \leq r \leq r_{2} \\ 4 \varepsilon\left[\left(\frac{\sigma}{r}\right)^{12}-\left(\frac{\sigma}{r}\right)^{6}\right], & \text { for } r>r_{2}\end{cases}
$$

with the fitting parameters found in Table 1, and the screening function $\varphi$ described in Equations 3 and 4 . 


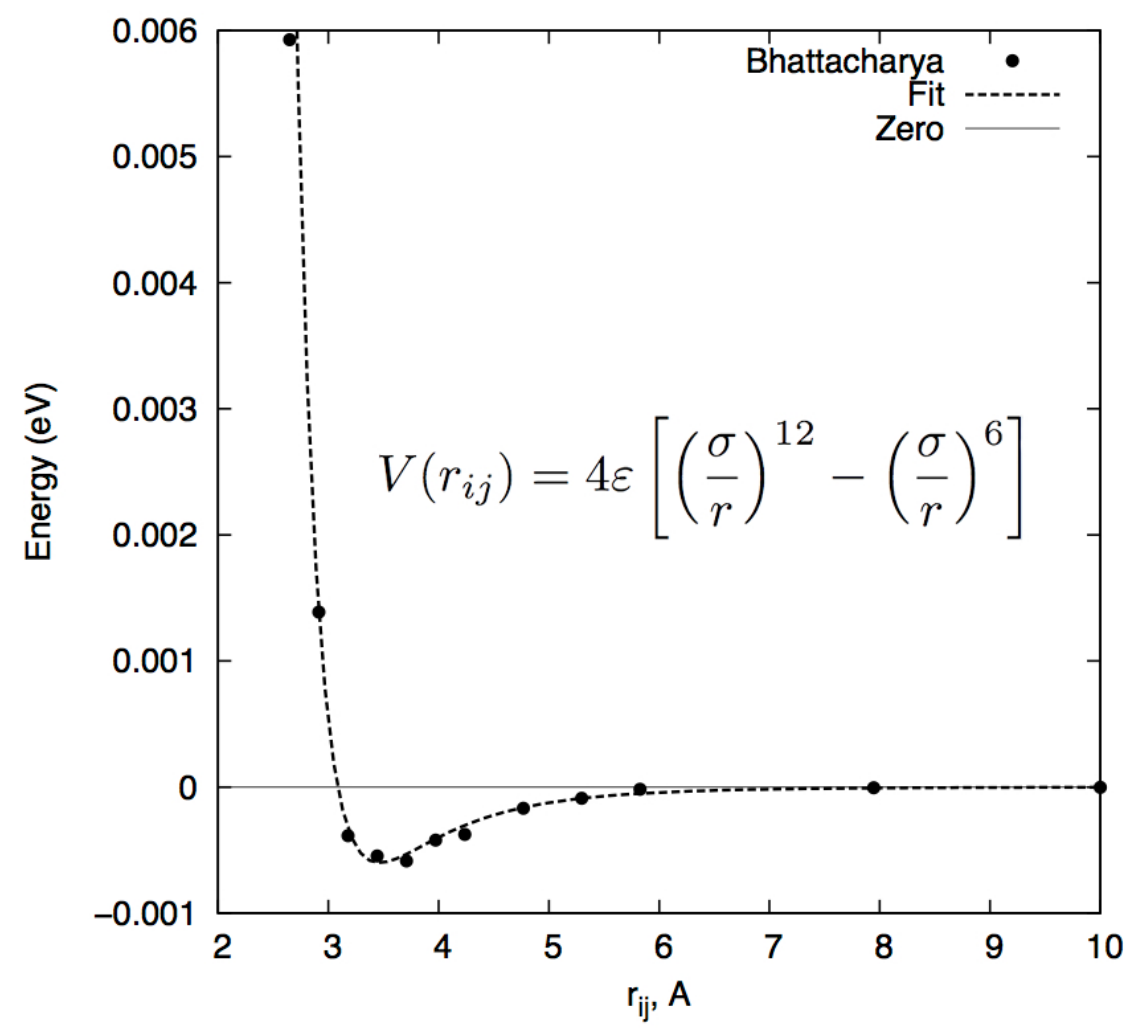

Figure 5: The data points are from Bhattacharya (1994), while the dashed line is our Lennard-Jones fit. The zero energy line is also shown for reference. Values of $\varepsilon=0.0006$ $\mathrm{eV}$ and $\sigma=3.0862 \AA$ are used.

Table 1: The parameters for the interatomic potential for hydrogen and helium referenced in Equation 6. Units are $\mathrm{eV}$ and $\AA$ where appropriate.

\begin{tabular}{cccccccc}
\hline$D_{0}$ & $D_{1}$ & $D_{2}$ & $D_{3}$ & $\sigma$ & $\varepsilon$ & $r_{1}$ & $r_{2}$ \\
\hline-0.318151 & 1.142826 & -0.530526 & 0.116766 & 3.0862 & 0.0006 & 0.5 & 2.5 \\
\hline
\end{tabular}




\section{CHAPTER II}

\section{SCREW DISLOCATION-DEFECT INTERACTIONS}

\subsection{Motivation and Literature Review}

The interactions of dislocations with intrinsic point defects are of importance in understanding the processes of plasticity, hardening [4], and irradiation creep. Plasticity is enabled through the presence of dislocations, which lower the stress needed to deform a material. Additionally, dislocations act as sinks for point defects, affecting dislocation growth and the subsequent swelling of irradiated materials.

The problem of modeling the effects of dislocations is inherently multiscale, and the motion of dislocations through climb, glide, slip, and cross-slip must be well understood on a microscopic scale in order for larger scale simulations to be properly parameterized $[112,60,100,89,46]$. There is a multi-decade history of computer simulation of dislocations; an excellent review of methods is found in Bulatov and Cai [13]. From ab initio and molecular dynamics simulations of single dislocation cores and individual point defects, parameters may be found which enable simulations of the interactions between dislocations themselves. Finally, properties found through discrete dislocation simulations, such as hardening parameters, may be used in large scale crystal plasticity models. There are several issues that must be considered when linking simulations of different scales, such as size effects $[158,94]$. McDowell [80] and Groh and Zbib [52] provide excellent reviews of the way in which multiscale simulations of dislocations and crystal plasticity may be structured, with the larger scale simulations relying on results from atomistics. Elasticity theory is one of a variety of inputs that are commonly used with the dislocation dynamics methodology. Groh and Zbib point out that a large number of discrete dislocation codes may only be properly applied to isotropic materials $[76,153]$. Isotropic elasticity is quite general and easy to implement, thus it is commonly used, if not entirely accurate. Exceptions to this include the anisotropic discrete dislocation code of Rhee et al. [122], which uses anisotropic 
expressions at least for short-range interactions, and that of Capolungo et al. for hcp metals [16]. The equations of anisotropic elasticity are much more complex than their isotropic counterparts and, aside from special cases [34], are often applicable to a particular orientation of dislocation and must be solved numerically. These factors necessitate an understanding of where and when theory and approximation may be used successfully, and where detailed effects of atomic structure must be known.

In both isotropic and anistropic continuum dislocation formulations, the strain field of the dislocation becomes singular at the core and must be treated carefully. There are clever solutions in the literature to get around this shortcoming, for example, Cai et al.'s nonsingular formulation [15], but they are not in widespread use. Clearly there is a need to characterize the deficiencies of isotropic theory as applied to highly anisotropic materials such as bcc iron, as well as to determine the limitations of the more powerful anisotropic formulation of continuum dislocation elasticity theory.

\subsubsection{Core Structure}

The determination of the proper structure of the core of a screw dislocation in bcc transition metals is a long standing problem [151]. In recent years, many multibody interatomic potentials have been developed for atomistic simulation of iron, but not all predict the same core structure for a $\frac{1}{2}\left[\begin{array}{lll}1 & 1 & 1\end{array}\right]$ screw dislocation. Some predict a degenerate structure, where the relaxed core spreads along three $\left\{\begin{array}{lll}1 & 1 & 0\end{array}\right\}$ planes of the $<\begin{array}{lllll}1 & 1>\text { zone. Two }\end{array}$ possible configurations exist, related by the symmetry operation of the $\left[\begin{array}{lll}1 & 0 & \overline{1}\end{array}\right]$ diad, and are equivalent in energy. Other potentials predict a compact structure, referred to as the non-degenerate core.

Until recently, the degenerate structure was accepted as accurate $[150,33]$. However, recent ab initio calculations [37] clearly show the non-degenerate structure to be preferred, and this structure is now believed to be the lowest energy configuration and a general feature of many transition metals [157]. One of the very few available embedded-atom method (EAM) potentials to predict this latter structure, that of Ackland et al. [3], is employed in this work. In fact, non-central forces are the physical cause for this configuration 


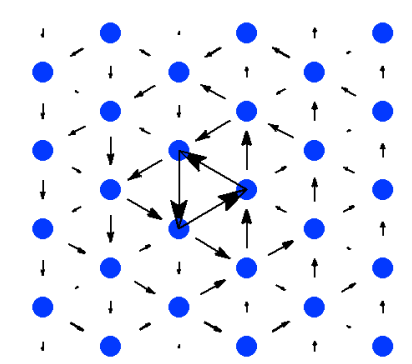

Figure 6: Differential displacement map of the screw core in iron; the non-degenerate structure is recovered.

[148, 149, 93, 51]; the Ackland potential does not explicitly account for this, while other types, including bond-order potentials, do [51]. However, this potential is widely used in the literature and does provide the appropriate core structure; the criteria under which a potential of this type may be guaranteed to generate this core type are described in [22]. Thus, it is useful for comparing the effects of atomic structure vs. those of elastic theory on dislocation-defect interactions. The differential displacement diagram [33] is used to verify that our computational setup results in the non-degenerate structure, as shown in Figure 6 . The point of this work is not to definitively determine the energetics of defect-dislocation interactions, but to understand at what point continuum elasticity theory reproduces atomistic results.

\subsubsection{Dislocation-Defect Interaction}

In this work we seek to intimately examine the differences in interactions between screw dislocations and point defects from linear elastic theory and atomistic simulation, in particular close to the core of a dislocation. The interactions between dislocations and point defects have been elucidated in a number of studies for both screw and edge dislocations using a variety of methods $[39,5,127,57]$, including tight-binding approximations $[78,79]$ and hybrid methods [129, 128], as well as studies of interactions with impurities [23] and clusters of self-interstitials $[75,111,110,154]$. Additionally, there have been a number of studies on dislocation glide via the double kink mechanism; different aspects of the nucleation and energy barriers have been simulated in a number of works $[150,30,20,48,19]$. We note that core spreading and kink nucleation will be assisted by the absorption of defects within 
the core. We endeavour to build upon this knowledge by conducting a detailed investigation of the direct interaction of a variety of orientations and types of point defects with a screw dislocation, including calculating interaction energies and performing stability analysis.

We calculate the interaction energy $E$ of intrinsic point defects (vacancies and interstitial dumbbells) with screw dislocations in alpha-iron. First, using the dipole force tensor and the dislocation strain field, we calculate the interaction energy using continuum methods

as discussed below. Second we perform atomistic calculations of the defect-dislocation interaction which incorporates the dislocation core explicitly. A defect is introduced near a dislocation, the energy of this ensemble of atoms minimized and the interaction energy is directly determined by comparing this energy to that of a defect far away from a dislocation. Of these two methods, the first does not contain any description of the dislocation core, while the other explicitly accounts for the atomic structure of the core. Thus, in order to determine the effects of the dislocation core structure, we compare these two methods of calculating the interaction energy.

\subsection{Linear Elasticity Theory}

Meissner et al. [82] derived the dislocation defect interaction energy from the dipole tensor and the dislocation strain field in a general anisotropic medium. This model was the basis of the work by Tomé et al. [144] in which the interaction energy in hexagonal close packed materials was studied near edge and screw dislocations. Both of these papers used lattice Green's functions to calculate the dipole tensors. Additional works invoking this theoretical framework and the model of point defects diffusion of Dederichs and Schroeder [28] include those of Tomé et al. [143], Monti et al. [88], and Smetniansky-de Grande et al. [130].

In this formulation, the interaction energy between the point defect and a dislocation separated by a distance $\mathbf{r}$ is given by

$$
E(\mathbf{r})=-\varepsilon_{i j}(\mathbf{r}) P_{i j}
$$

where $\varepsilon$ is the strain field of the dislocation and $P_{i j}$ are components of the dipole force tensor $\mathbf{P}$, which will be discussed in the following section. ${ }^{1}$ The strain field of the dislocation 
can be calculated from the displacement field as:

$$
\varepsilon_{i j}=\frac{1}{2}\left(\frac{\partial u_{i}}{\partial x_{j}}+\frac{\partial u_{j}}{\partial x_{i}}\right)
$$

At large separation distances, where the defect is far enough away from the dislocation to feel no influence, the interaction energy $E=0$.

When we wish to compare to atomistics, it is convenient to add another term to Equation 7 , so that

$$
E(\mathbf{r})=-\varepsilon_{i j}(\mathbf{r}) P_{i j}+E_{\text {defect }} .
$$

$E_{\text {defect }}$ is an additional energy accounting for different types of configurations of the same type of defect. This term is especially relevant for interstitial dumbbells. In bcc iron, the $<110>$ dumbbell configuration is the lowest energy interstitial defect. Other orientations are higher energy structures, so the addition of $E_{\text {defect }}$ for the higher energy structures, relative to the lowest energy structure, allows all interaction energies for a given type of defect to be compared on equal footing. This term is equal to the additional formation energy required to form the defect of interest, above what is required for a $<110>$ dumbbell. With the interatomic potential we are using, the formation energy for a $<110>$ dumbbell is $3.53 \mathrm{eV}, 4.34 \mathrm{eV}$ for a $<100>$ dumbbell, and $4.02 \mathrm{eV}$ for a $<111>$ dumbbell [83]. Thus, $E_{\text {defect }}^{<110>}=0.0 \mathrm{eV}, E_{\text {defect }}^{<111>}=0.49 \mathrm{eV}$ and $E_{\text {defect }}^{<100}=0.81 \mathrm{eV}$. In this way, all interaction energies for interstitials can be compared directly.

\subsubsection{Dipole Tensor}

The dipole force tensor describes the influence that a point defect, either a vacancy or interstitial, has on its neighbors in otherwise perfect bulk material. It may be calculated using different methods [47]; in this case it is calculated by

$$
P_{i j}=\sum_{k=1}^{N}\left[S_{i}^{(k)}+d_{i}^{(k)}\right] F_{j}^{(k)},
$$

\footnotetext{
${ }^{1}$ It is important to make sure all calculations and comparisons are done in the same coordinate system. For example, dipole tensors are given in the crystal coordinate system, while strain fields are given, and atomistics are performed, in the dislocation coordinate system. First and second order tensors can be transformed between the crystal and dislocation systems by $\mathbf{v}^{C}=\mathbf{T} \mathbf{v}^{D}$, and $\mathbf{P}^{C}=\mathbf{T} \mathbf{P}^{D} \mathbf{T}^{\mathbf{T}}$ where $\mathbf{T}$ is the transformation matrix, which contains the normalized vectors of the dislocation coordinate system as its columns.
} 
Table 2: The components of the dipole tensor $\mathbf{P}$ for a variety of point defects, expressed in crystal axes.

\begin{tabular}{|c|c|c|c|c|c|c|}
\hline defect type & $P_{11}$ & $P_{22}$ & $P_{33}$ & $P_{12}$ & $P_{13}$ & $P_{23}$ \\
\hline vacancy & -2.7119 & -2.7119 & -2.7119 & 0.00 & 0.00 & 0.00 \\
\hline$\left[\begin{array}{lll}1 & 1 & 0\end{array}\right]$ dumbbell & 10.2056 & 10.2056 & 10.9080 & 4.7428 & 0.00 & 0.00 \\
\hline$\left[\begin{array}{lll}1 & 0 & 0\end{array}\right]$ dumbbell & 8.7977 & 8.1689 & 8.1689 & -1.0332 & -1.0332 & 2.9766 \\
\hline$\left[\begin{array}{llll}1 & 1 & 1\end{array}\right]$ dumbbell & 8.5493 & 8.5493 & 8.5493 & 4.6977 & 4.6977 & 4.6977 \\
\hline
\end{tabular}

where the summation is over $N$ neighbors of the defect, $\mathbf{S}$ denotes the perfect lattice position of a neighbor with respect to the defect, $\mathbf{d}$ is the displacement from the perfect lattice position caused by the defect's presence, and the Kanzaki force, F, is defined as the force that must be applied to maintain the displaced structure when the defect is removed. The Kanzaki force is equivalently the component of the total force that the defect exerts on a neighbor when in the relaxed configuration. For a specific potential these quantities can be calculated with Green function methods or directly with molecular statics.

In this work, the latter method is used. Atomistic simulations are performed in which a defect is introduced into a perfect lattice. Conjugate gradient minimization is used to relax the lattice. Care must be taken that the lattice is fully relaxed; this is ensured by requiring that no component of force on any atom is larger than $1 \times 10^{-9} \mathrm{eV} / \AA$. An interstitial dumbbell with the neighboring atoms after relaxation is depicted in Figure 7(a). Then, the defect is removed and the lattice restored to its original state, but with the neighbors maintaining their displacements, as shown in Figure 7(b). The force produced by the defect site on the neighbors can then be calculated; it is equal in magnitude but opposite in sign to the force produced by the defect itself. In the calculations which follow, we use the LAMMPS molecular dynamics code [113] and the clsman atomistic code developed at Los Alamos National Laboratory. The six independent components of the symmetric tensors $\mathbf{P}$ are given in Table 2 for four types of defects: the vacancy and three configurations of the

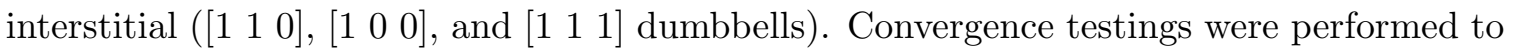
ensure that a sufficient number of neighbor shells were included in the calculations; at least nine neighbor shells (approx. $2.5 \AA$ ) beyond the defect were included. 


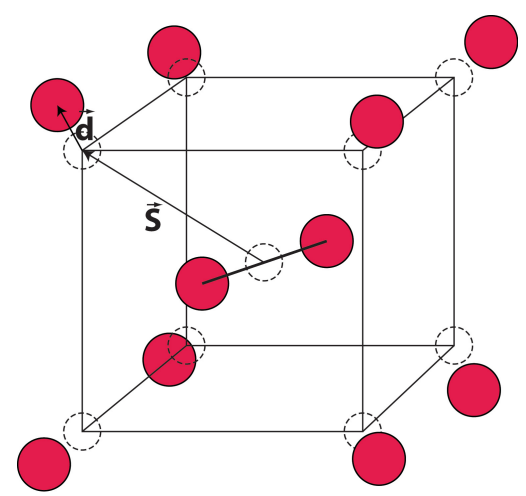

(a) A defect displaces its neighbors by a distance $\mathbf{d}$ when the system is relaxed.

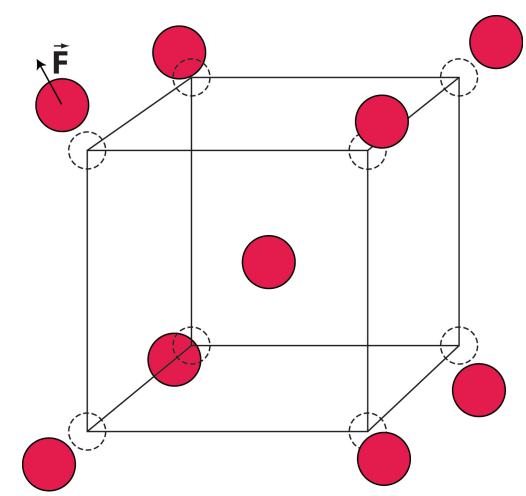

(b) When the defect is removed, a force $\mathbf{F}$ is required to hold the neighbors in their displaced positions.

Figure 7: Schematic of the quantities used when calculating the dipole tensor for a generic defect in a bcc lattice. Dotted circles denote perfect lattice positions. Red circles denote displaced atom positions caused by the presence of the defect.

\subsubsection{Strain Fields}

The strain field may be formulated isotropically or anisotropically. The isotropic formulation is well-known and generally may be applied to any generic screw dislocation. On the other hand, the anisotropic formulation must be derived for a particular orientation of the dislocation.

For any isotropic medium, a screw dislocation results in displacement along the $x_{3}$ direction only, given by:

$$
u_{3}=\frac{b_{x_{3}}}{2 \pi} \tan ^{-1} \frac{x_{2}}{x_{1}}
$$

where $b_{x_{3}}$ is the magnitude of the Burgers vector and $\xi$ lies along the $x_{3}$ axis [60]. There are no displacements in the directions perpendicular to the dislocation line. Thus, $\varepsilon_{11}=$ $\varepsilon_{22}=\varepsilon_{33}=\varepsilon_{12}=0$, and the non-zero symmetric strain field components are

$$
\begin{aligned}
& \varepsilon_{13}=-\frac{b_{x_{3}}}{4 \pi r^{2}} x_{2} \\
& \varepsilon_{23}=\frac{b_{x_{3}}}{4 \pi r^{2}} x_{1} .
\end{aligned}
$$

For the anisotropic formulation, the equations needed to generate a $<1 \quad 1 \quad 1>$ screw dislocation are given by Hirth [59], based on the solutions of Eshelby, Read, and Shockley [34], Stroh [136], and Head [56]. 
Note that the equations in this section apply to the coordinate system where $x_{1}=\left[\begin{array}{ll}\overline{1} & 2 \\ 1\end{array}\right]$,

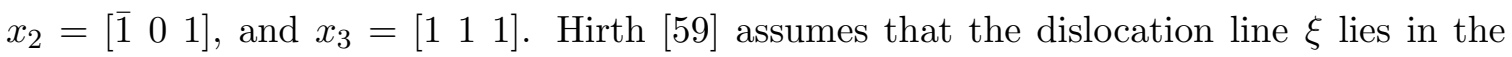
negative $x_{3}$ direction, that is, the $\left[\begin{array}{lll}\overline{1} & \overline{1} & \overline{1}\end{array}\right]$ direction. The equations can be used to describe right or left handed dislocations, with a negative or positive Burgers vector, respectively.

The elastic constants in the dislocation coordinate system $C_{i j}$ are defined in terms of the standard elastic constants $C_{i j}^{0}$ from the crystal coordinate system, and in terms of the anisotropy factor $H=2 C_{44}^{0}-C_{12}^{0}-C_{11}^{0}$. Their values are given by

$$
\begin{aligned}
& C_{11}=C_{11}^{0}+H / 2 \quad C_{44}=C_{44}^{0}-H / 3 \\
& C_{12}=C_{12}^{0}-H / 6 \quad C_{66}=C_{44}^{0}-H / 6 \\
& C_{13}=C_{12}^{0}-H / 3 \quad C_{15}=-\sqrt{2} H / 6 \\
& C_{33}=C_{11}^{0}+2 H / 3 \quad 2 C_{66}=C_{11}-C_{12} .
\end{aligned}
$$

For a right handed dislocation in the $x_{3}$ direction Hirth gives the displacements as

$$
\begin{aligned}
u_{1}= & -\frac{b_{x_{3}}}{6 \pi}\left\{\tan ^{-1}\left(\frac{F x_{2}}{x_{1}}\right)-\frac{1}{2} \tan ^{-1}\left(\frac{4 F x_{2}}{J_{1} x_{1}-\sqrt{3} J_{3} x_{2}}\right)-\frac{1}{2} \tan ^{-1}\left(\frac{4 F x_{2}}{J_{1} x_{1}+\sqrt{3} J_{3} x_{2}}\right)\right\} \\
& \times(A+B)-\frac{\sqrt{3} b_{x_{3}}}{12 \pi}\left\{\ln \left(\frac{J_{1} x_{1}^{2}+J_{2} x_{2}^{2}-2 \sqrt{3} J_{3} x_{1} x_{2}}{J_{1}}\right)^{1 / 2}\right. \\
& \left.-\ln \left(\frac{J_{1} x_{1}^{2}+J_{2} x_{2}^{2}+2 \sqrt{3} J_{3} x_{1} x_{2}}{J_{1}}\right)^{1 / 2}\right\} \times B F \\
u_{2}= & -\frac{\sqrt{3} b_{x_{3}}}{12 \pi}\left\{\tan ^{-1}\left(\frac{4 F x_{2}}{J_{1} x_{1}-\sqrt{3} J_{3} x_{2}}\right)-\tan ^{-1}\left(\frac{4 F x_{2}}{J_{1} x_{1}+\sqrt{3} J_{3} x_{2}}\right)\right\} \times(A+B) \\
& -\frac{b_{x_{3}}}{6 \pi}\left\{\ln \left(x_{1}^{2}+F^{2} x_{2}^{2}\right)^{1 / 2}-\frac{1}{2} \ln \left(\frac{J_{1} x_{1}^{2}+J_{2} x_{2}^{2}-2 \sqrt{3} J_{3} x_{1} x_{2}}{J_{1}}\right)^{1 / 2}\right. \\
& \left.-\frac{1}{2} \ln \left(\frac{J_{1} x_{1}^{2}+J_{2} x_{2}^{2}+2 \sqrt{3} J_{3} x_{1} x_{2}}{J_{1}}\right)^{1 / 2}\right\} \times B F \\
u_{3}= & -\frac{b_{x_{3}}}{6 \pi}\left\{\tan ^{-1}\left(\frac{F x_{2}}{x_{1}}\right)+\tan ^{-1}\left(\frac{4 F x_{2}}{J_{1} x_{1}-\sqrt{3} J_{3} x_{2}}\right)+\tan ^{-1}\left(\frac{4 F x_{2}}{J_{1} x_{1}+\sqrt{3} J_{3} x_{2}}\right)\right\}
\end{aligned}
$$


where

$$
\begin{array}{rlrl}
F & =\frac{(R+1)^{1 / 3}+(R-1)^{1 / 3}}{(R+1)^{1 / 3}-(R-1)^{1 / 3}} & R^{2}=\frac{C_{66}\left(C_{11} C_{44}-C_{15}^{2}\right)}{C_{11}\left(C_{44} C_{66}-C_{15}^{2}\right)} \\
J_{1}=1+3 F^{2} & J_{6}=C_{44} / C_{15} \\
J_{2}=3+F^{2} & J_{7}=\left(C_{15} S_{44}\right)^{-1} \\
J_{3}=1-F^{2} & J_{8}=\left(F^{2} C_{11}+C_{12}\right)^{-1} \\
J_{4}=\left(C_{12}+C_{66}\right) /\left(C_{15} S_{44}\right) & A=J_{6}-\left(J_{2} J_{7} / J_{1}\right) \\
J_{5}=\left[C_{44}\left(C_{12}+C_{66}\right)-2 C_{15}^{2}\right] / C_{15} & B=J_{8}\left[\left(J_{2} J_{4} / J_{1}\right)-J_{5}\right] \\
S_{44}=C_{66} /\left(C_{44} C_{66}-C_{15}^{2}\right) . &
\end{array}
$$

Using Equation 8, we can calculate the components of the strain field:

$$
\begin{aligned}
& \frac{\partial u_{1}}{\partial x_{1}}=-\frac{b_{x_{3}}}{6 \pi}\left\{\frac{2 F J_{1} x_{2}}{D_{+}}+\frac{2 F J_{1} x_{2}}{D_{-}}-\frac{F x_{2}}{G}\right\} \times(A+B)-\frac{\sqrt{3} b_{x_{3}}}{12 \pi}\left\{\frac{K_{-}}{M_{-}}-\frac{K_{+}}{M_{+}}\right\} \times B F \\
& \frac{\partial u_{1}}{\partial x_{2}}=-\frac{b_{x_{3}}}{6 \pi}\left\{-\frac{2 F J_{1} x_{1}}{D_{+}}-\frac{2 F J_{1} x_{1}}{D_{-}}+\frac{F x_{1}}{G}\right\} \times(A+B)-\frac{\sqrt{3} b_{x_{3}}}{12 \pi}\left\{\frac{L_{-}}{M_{-}}-\frac{L_{+}}{M_{+}}\right\} \times B F \\
& \frac{\partial u_{2}}{\partial x_{1}}=-\frac{b_{x_{3}}}{6 \pi}\left\{\frac{K_{+}}{2 M_{+}}+\frac{K_{-}}{2 M_{-}}-\frac{x_{1}}{G}\right\} \times(B F)-\frac{\sqrt{3} b_{x_{1}}}{12 \pi}\left\{\frac{4 F J_{1} x_{2}}{D_{+}}-\frac{4 F J_{1} x_{2}}{D_{-}}\right\} \times(A+B) \\
& \frac{\partial u_{2}}{\partial x_{2}}=-\frac{b_{x_{3}}}{6 \pi}\left\{\frac{L_{+}}{2 M_{+}}+\frac{L_{-}}{2 M_{-}}-\frac{F^{2} x_{2}}{G}\right\} \times(B F)-\frac{\sqrt{3} b_{x_{3}}}{12 \pi}\left\{\frac{4 F J_{1} x_{1}}{D_{-}}-\frac{4 F J_{1} x_{1}}{D_{+}}\right\} \times(A+B) \\
& \frac{\partial u_{3}}{\partial x_{1}}=-\frac{b_{x_{3}}}{6 \pi}\left\{-\frac{4 F J_{1} x_{2}}{D_{+}}-\frac{4 F J_{1} x_{2}}{D_{-}}-\frac{F x_{2}}{G}\right\} \\
& \frac{\partial u_{3}}{\partial x_{2}}=-\frac{b_{x_{3}}}{6 \pi}\left\{\frac{4 F J_{1} x_{1}}{D_{-}}+\frac{4 F J_{1} x_{1}}{D_{+}}+\frac{F x_{1}}{G}\right\}
\end{aligned}
$$

where

$$
\begin{aligned}
L_{ \pm} & =J_{2} x_{2} \pm \sqrt{3} J_{3} x_{1} \\
K_{ \pm} & =J_{1} x_{1} \pm \sqrt{3} J_{3} x_{2} \\
M_{ \pm} & =J_{1} x_{1}^{2}+J_{2} x_{2}^{2} \pm 2 \sqrt{3} J_{3} x_{1} x_{2} \\
D_{ \pm} & =16 F^{2} x_{2}^{2}+K_{ \pm} \\
G & =\left(x_{1}^{2}+F^{2} x_{2}^{2}\right)^{-1}
\end{aligned}
$$

and $\partial u_{1} / \partial x_{3}=\partial u_{2} / \partial x_{3}=\partial u_{3} / \partial x_{3}=0$.

The elastic constants we use in our calculations have the values $C_{11}^{0}=243.4 \mathrm{GPa}$, $C_{12}^{0}=145.0 \mathrm{GPa}$, and $C_{44}^{0}=116.0 \mathrm{GPa}$. These numbers come from the interatomic 


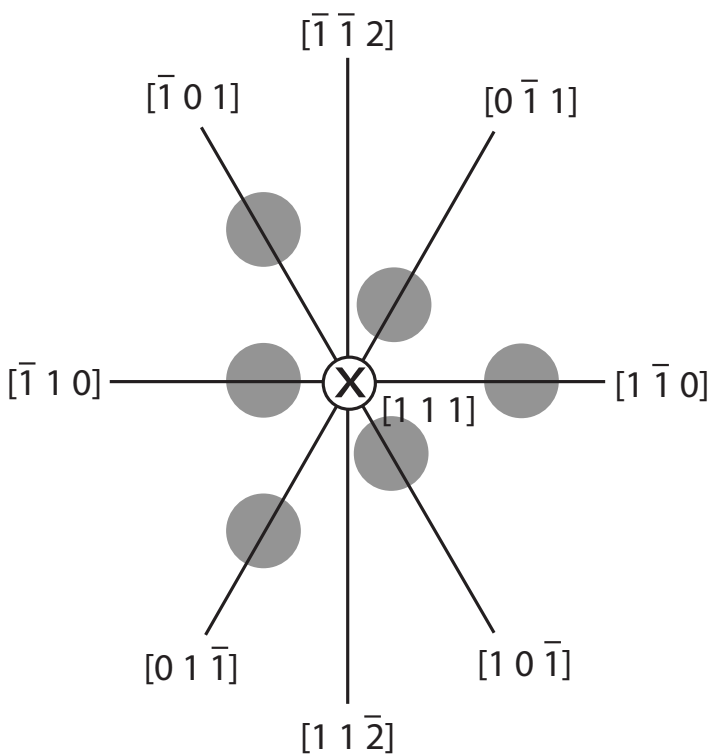

Figure 8: The coordinate system for the screw dislocation, with the $\left[\begin{array}{lll}1 & 1 & 1\end{array}\right]$ direction pointing into the page. The orientation of all figures in this paper correspond to this system. The dislocation line $\xi$ and the Burgers vector $\mathbf{b}$ point along the $\left[\begin{array}{lll}1 & 1 & 1\end{array}\right]$ direction, resulting in a right-handed dislocation. The atoms that comprise the core are shown as grey circles.

potential we use in this work, where they were parameters used in the fitting procedure $[83]$.

\subsection{Atomistic Simulation}

The coordinate system used in describing our results is shown in Fig. 8. The [1 11 1] direction is into the page. Both the dislocation line vector $\xi$ and the Burgers vector $\mathbf{b}$ point in the same direction along the $x_{3}$ axis, resulting in a right-handed dislocation.

Our system is cylindrical, with periodic boundary conditions in the direction of the dislocation line. The box size is approximately $37.1 \AA$ in the periodic direction, and the cylinder has a radius of approximately $100 \AA$. Initial displacement of all atoms is performed according to linear elastic theory. An outer shell of atoms (approximately $20 \AA$ ) is then frozen, while the core is allowed to relax. The resulting core structure was identical when either isotropic or anisotropic displacement expressions were used for the initial displacements.

We introduce defects into the simulation box in a systematic way. First, an individual iron atom is replaced by a defect, either by removing it for a vacancy, or shifting it and 
introducing an additional atom for an interstitial dumbbell. The energy of the system is minimized, while keeping the outer shell frozen, to obtain the relaxed structure containing the defect. The energy of the system is then measured. This process is repeated, starting from the initial dislocation structure without defects, for each unique atomic site within the simulation box. In this way, the interaction energy can be calculated between a defect and the dislocation as a function of their relative positions.

\subsection{Results}

Interaction energy from atomistics is calculated by the following formula:

$$
E_{\text {int }}=\left[E_{D}^{d}+E_{0}\right]-\left[E_{D}+E_{0}^{d}\right]
$$

All energies are total energies of the ensemble system, where the $D$ subscript indicates a system containing a dislocation, the 0 subscript indicates a single crystal system, and a $d$ superscript indicates that the defect of interest is contained in the system. The energy of the system containing both the dislocation and the defect given by atomistic calculation $\left(E_{D}^{d}\right)$ includes the energy of formation for both the dislocation and the defect. Thus, in order to extract out just the interaction energy, the last two terms must be subtracted. Additionally, $E_{0}$ is added back to the equation for balance of atoms. With this definition, a negative interaction indicates that the defect is attracted to the dislocation.

Our system contains 100095 atoms, of which 36090 are frozen, before defects are introduced. Thus, $E_{0}=-399082.256 \mathrm{eV}$ and $E_{D}=-399043.396 \mathrm{eV}$. $E_{0}^{d}$ is dependent on the type of defect. For a vacancy, $E_{0}^{d}(V)=-399076.522 \mathrm{eV}$. For all types of interstitials considered, the interaction energies are relative to the lowest energy interstitial in the bulk crystal, the $<110>$ dumbbell, which gives $E_{0}^{d}(I)=-399082.740 \mathrm{eV}$. This also allows for

direct comparison of the energies between all atomistic results. $E_{D}^{d}$ is determined by the atomistic results for each position and defect.

Note that in all figures showing basic interaction energies for atomistic data, the interaction energy scale bar runs from $-0.5 \mathrm{eV}$ to $0.5 \mathrm{eV}$, even when most of the data for a particular figure does not approach these limits. This was done so that interaction energies from atomistics could be easily intuitively compared across all defect configurations. In the 
remainder of this section, we will describe the results from atomistic and continuum theory for $<110>,<111>$, and $<100>$ dumbbell configurations, and for vacancies.

\subsection{1 $<110>$ Dumbbell}

The $<110>$ type dumbbell is known to be the most stable interstitial configuration in the bulk in bcc iron, in contrast to many other bcc metals in which crowdions are the most stable configuration. In our dislocation coordinate system, not all members of the $<110>$ family are created equal with respect to their orientation to the dislocation. A $\left[\begin{array}{lll}1 & \overline{1} & 0\end{array}\right]$ dumbbell lies perpendicular to the dislocation line, along the $x$-axis of figure 8 , while a $\left[\begin{array}{lll}1 & 1 & 0\end{array}\right]$ dumbbell makes an angle of $\sim 35^{\circ}$ with the core. We define

$$
<\begin{array}{lll}
\overline{1} & 0
\end{array}>\left\{\left[\begin{array}{lll}
1 & \overline{1} & 0
\end{array}\right],\left[\begin{array}{lll}
1 & 0 & \overline{1}
\end{array}\right],\left[\begin{array}{lll}
0 & 1 & \overline{1}
\end{array}\right],\left[\begin{array}{lll}
\overline{1} & 1 & 0
\end{array}\right],\left[\begin{array}{lll}
\overline{1} & 0 & 1
\end{array}\right],\left[\begin{array}{lll}
0 & \overline{1} & 1
\end{array}\right]\right\}
$$

to differentiate those specific dumbbells which are perpendicular to the dislocation line from those of the general $<110>$ family, and use

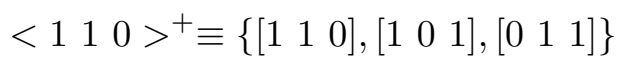

to indicate only those members which have all positive components, and thus have some extent along the core.

We begin by calculating energetics for $\left[\begin{array}{lll}1 & \overline{1} & 0\end{array}\right]$ dumbbells; the results are shown in Figure 9. The dumbbell lies on the horizontal axis, with no extent into the page. A clear pattern of positive and negative interaction energies emerges from the atomistic simulations. The most negative interaction energies are seen to the left of the core, while to the right, the most positive energies are seen. Bands of slightly negative interaction energy are seen in the $\left[\begin{array}{lll}0 & \overline{1} & 1\end{array}\right]$ and $\left[\begin{array}{lll}1 & 0 & \overline{1}\end{array}\right]$ directions, and slightly positive bands are seen in the $\left[\begin{array}{lll}\overline{1} & 0 & 1\end{array}\right]$ and $\left[\begin{array}{lll}0 & 1 & \overline{1}\end{array}\right]$ directions. This pattern reflects the non-degenerate core structure of the screw dislocation in bcc iron. Even at the angles at which the long-range interaction energy is positive (repulsive), within about 5-8 $\AA$ of the core, all interaction energies are negative. This indicates that if a defect travels close enough to the core from any direction, it will ultimately be attracted instead of repelled. As can be seen in Figure 9, there are five lattice sites in the core (white area) at which the dumbbell is unstable and is spontaneously 
absorbed into the core under minimization. This results in a much lower energy structure, off the scale in Figure 9, which will be discussed later.

When we compare these atomistic results to those from elasticity theory, we find several discrepancies. The anisotropic strain field is able to replicate the pattern of negative and positive interaction energies (given by Eq. 9) around the core, although the magnitude of the interactions is underestimated. Also, anisotropic elasticity theory does not predict the negative energies very near the core in all directions that we see from atomistics. The absolute error and absolute percentage error (referenced to the atomistic solution) between atomistics and anisotropic elasticity theory can be seen in Figure 10. To the right and left of the core, where the interaction energies have the largest magnitude, the absolute error is greatest. However, these areas have some of the lowest relative errors. In areas of very small magnitude interaction energy, the percentage error is very significant, but errors in these regions would be of little practical significance. In the core, as we would expect, the error approaches $1 \mathrm{eV}$, but outside of the core the error rarely exceeds $0.1 \mathrm{eV}$. Even so, the percentage errors are quite large (between about 10 and $50 \%$ in significant regions of strong interaction energy). Overall, anisotropic theory agrees qualitatively with atomistics outside of the core region, but has large errors quantitatively.

The results from anisotropic theory are significantly better than those from isotropic theory (Figure 9). Isotropic theory simply predicts a clear divide between positive and negative interaction energies at the $y$-axis and does not account for the non-degenerate core structure at all.

These results can be generalized for any $<1 \overline{1} 0>$ dumbbell. The pattern of interaction energy seen in Figure 9 will appear rotated by $120^{\circ}$ for other dumbbells of this type, with the strongest positive and negative interaction energies appearing when the dumbbell is pointed directly towards the core.

A $\left[\begin{array}{lll}1 & 1 & 0\end{array}\right]$ dumbbell was also examined; results are shown in Figure 11. This dumbbell displays three regions of negative interaction energy, and three regions of positive/repulsive interaction. In contrast to the $\left[\begin{array}{lll}1 & \overline{1} & 0\end{array}\right]$, however, all three regions are the same size, displaying a symmetry when looking down the dislocation line. Additionally, the magnitudes of the 


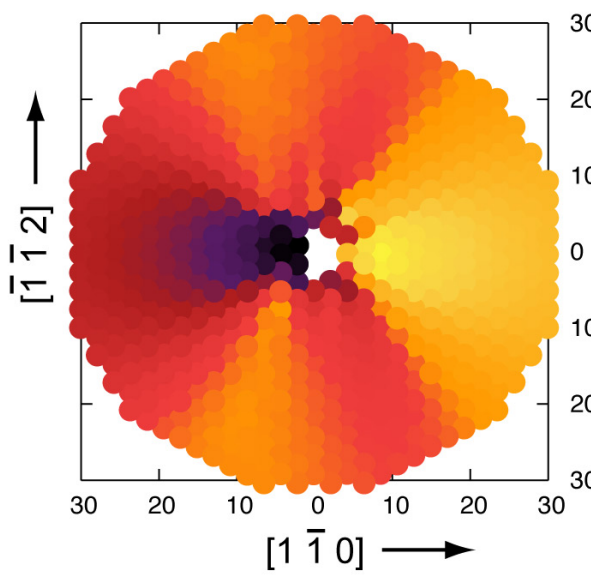

(a)

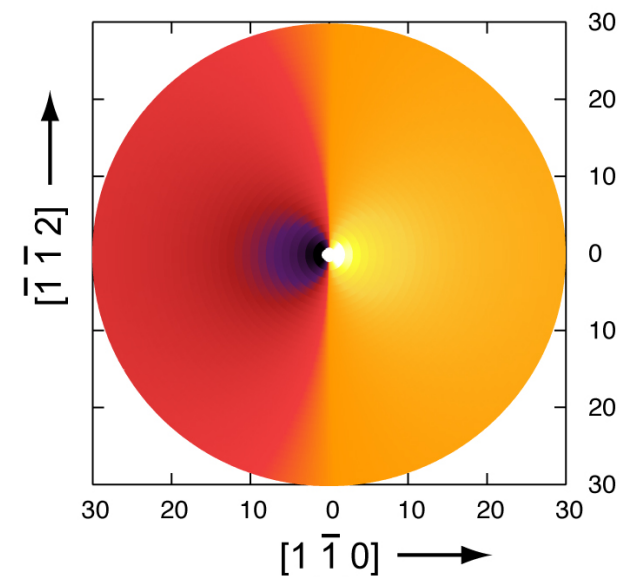

(c)

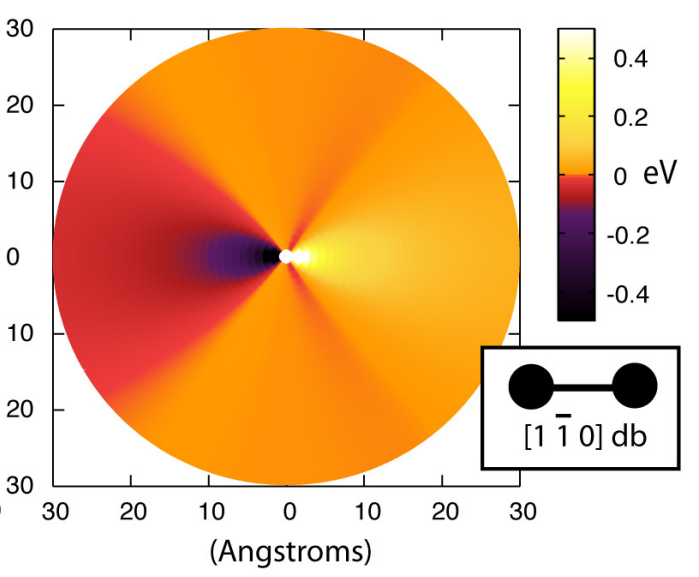

(b)

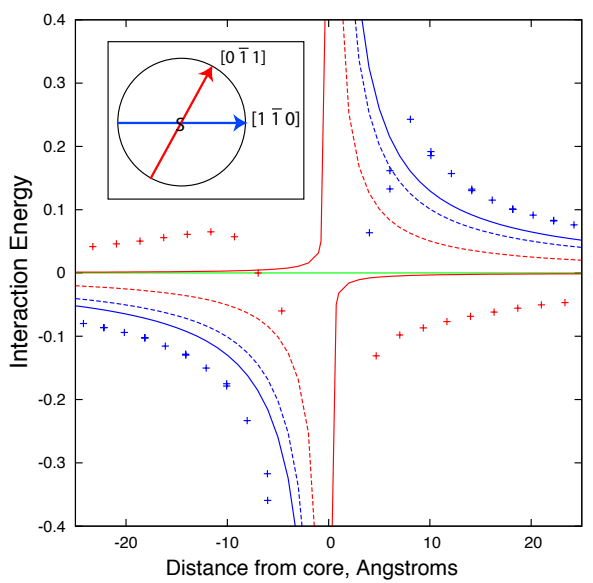

(d)

Figure 9: Plots of the interaction energy of a $\left[\begin{array}{lll}1 & \overline{1} & 0\end{array}\right]$ dumbbell with the dislocation core as a function of distance and angle from the dislocation. In all figures, the core is centered at $(0,0)$, and the numbers on the axes show distance from the core in Angstroms. Interaction energies are plotted by color, in $\mathrm{eV}$. (a) shows the interaction energies calculated directly from atomistics. (b) shows $E$ calculated with elasticity theory, using Equation 9 with the anisotropic strain field, with the dipole tensor $\mathbf{P}$ calculated using atomistic methods. (c) shows the interaction energy calculated with elasticity theory using the isotropic strain field. (d) shows profiles of the interaction energy along the directions indicated in the inset circle. As the interstitial position varies along the $\left[\begin{array}{lll}1 & \overline{1} & 0\end{array}\right]$ direction (blue) and the $\left[\begin{array}{lll}0 & \overline{1} & 1\end{array}\right]$ direction (red) the interaction energy is plotted. Dotted lines indicate isotropic results, solid lines indicate anisotropic results, and points show atomistic results. The anisotropic strain field results in interactions of the same sign as those shown by atomistics, even when the magnitude is in disagreement; however the results when using the isotropic strain field does not even have this property. 


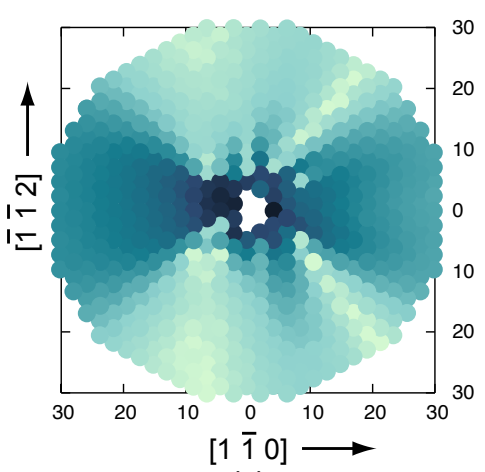

(a)

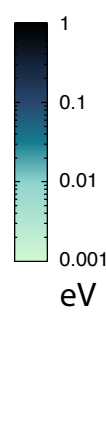

$\mathrm{eV}$

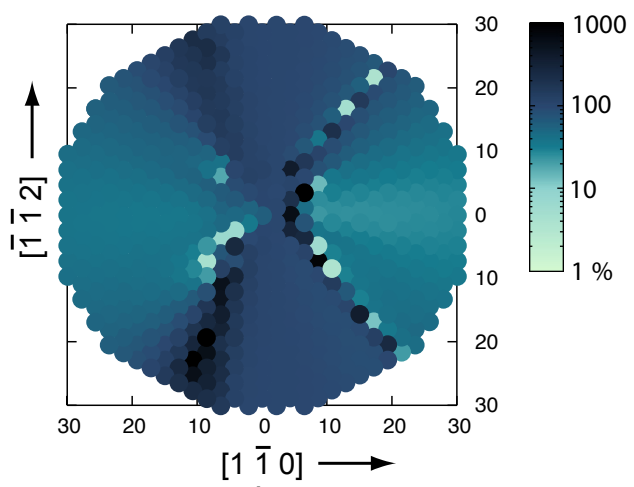

(b)

Figure 10: (a) shows the absolute error in $\mathrm{eV}$ between the atomistic and anisotropic elasticity theory results for the $\left[\begin{array}{lll}1 & \overline{1} & 0\end{array}\right]$ dumbbell. (b) shows the absolute value of the percent error between the atomistic and anisotropic elasticity theory results with respect to the atomistic calculations for the same dumbbell. The same coordinate system that is used in Figure 9 is used here.

interactions are weaker than for the $\left[\begin{array}{lll}1 & \overline{1} & 0\end{array}\right]$ dumbbell. Essentially, this indicates that a member of the $<1 \overline{1} 0>$ family is energetically preferred over a $<1 \quad 10>^{+}$in the regions where negative interaction energy is seen (the $\left[\begin{array}{lll}1 & 1 & 0\end{array}\right],\left[\begin{array}{lll}1 & 0 & \overline{1}\end{array}\right]$, and $\left[\begin{array}{lll}0 & \overline{1} & 1\end{array}\right]$ directions). However, in the other regions where a positive interaction is observed, a $<110>^{+}$may be preferred since its interaction is less repulsive than that of a $<1 \overline{1} 0>$.

\section{$2.4 .2<111>$ Dumbbell}

The $<111>$ family of dumbbells is not as energetically favorable in the bulk as the $<110>$, but more so than the $<100>$ family. However, the pattern of energetic favorability may change near a strong strain field such as that of a dislocation. [ [ $\left.\begin{array}{lll}1 & 1 & 1\end{array}\right]$ dumbbells, which lie parallel to the dislocation line, are introduced at each lattice site about our screw dislocation core (one at a time) and subsequently minimized.

After an analysis of the minimized structures, it is clear that the $\left[\begin{array}{lll}1 & 1 & 1\end{array}\right]$ dumbbell is not a stable configuration everywhere, as illustrated in Figure 12. We map the final dumbbell type by color, using an algorithm that can distinguish between families of directions, but does not account for permutations within a family (for example, a $\left[\begin{array}{lll}1 & 1 & 0\end{array}\right]$ and a $\left[\begin{array}{lll}1 & \overline{1} & 0\end{array}\right]$ will both appear green). We find that half of the dumbbells convert to $<110>$-type structures, while the other half lie somewhere between $<\begin{array}{llllll}1 & 1>\text { and }<2 & 1 & 1\end{array}$; they have a $<111>$ 


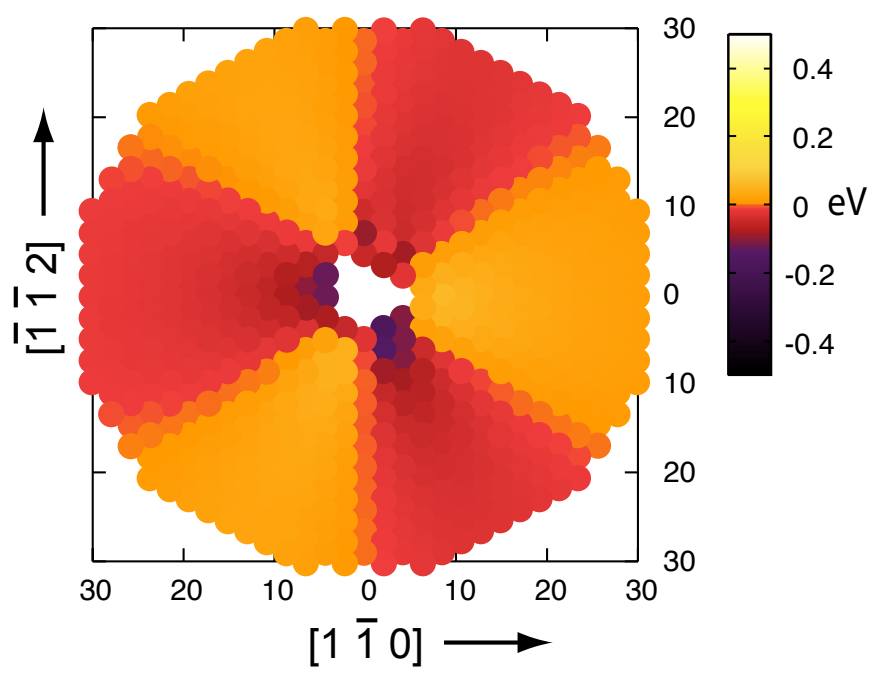

Figure 11: The interaction energies between a [ $\left.\begin{array}{lll}1 & 1 & 0\end{array}\right]$ dumbbell and a screw dislocation core are shown as a function of of distance and angle from the dislocation core. The same conventions as in Figure 9 are used. Note that the magnitude of interactions is not as strong as for the $\left[\begin{array}{lll}1 & \overline{1} & 0\end{array}\right]$ orientation.

that is slightly twisted to conform with the helical structure of the screw dislocation. This can be explained by comparing to our above results for $\left[\begin{array}{lll}1 & \overline{1} & 0\end{array}\right]$ dumbbells.

In the regions where it is most energetically favorable to have a dumbbell from the

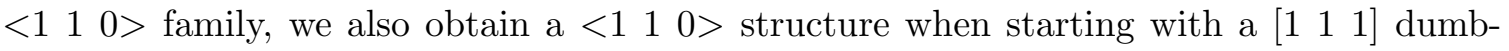
bell structure. Looking at individual dumbbells from the $<110>$ regions, we find that dumbbells convert to $\left\langle 110>^{+}\right.$structures instead of $\langle 1 \overline{1} 0>$, due to the smaller degree of rotation needed, or the relative closeness of the two structures. In the regions where a $<110>$ dumbbell was shown to have a positive interaction energy with respect to the dislocation, [ [ $\left.\begin{array}{lll}1 & 1 & 1\end{array}\right]$ dumbbells rotate away from being perfectly aligned with the core, but do not convert to $<1 \quad 1 \quad 0>$-type. Dumbbells very near the core appear to stay in $\left[\begin{array}{lll}1 & 1 & 1\end{array}\right]$ configurations, but this is really an artifact of their being absorbed into the core, creating a crowdion-like structure along the dislocation line.

Interaction energies from atomistics for the $\left[\begin{array}{lll}1 & 1 & 1\end{array}\right]$ dumbbell calculations can be seen in Figure 13. As described above, all interaction energies are referred to the $<110>$ structure, so results from all atomistics calculations of interstitials can be compared directly. In the regions where dumbbells rotated to the $\left[\begin{array}{lll}1 & 1 & 0\end{array}\right]$ shape, interaction energies are negative. 


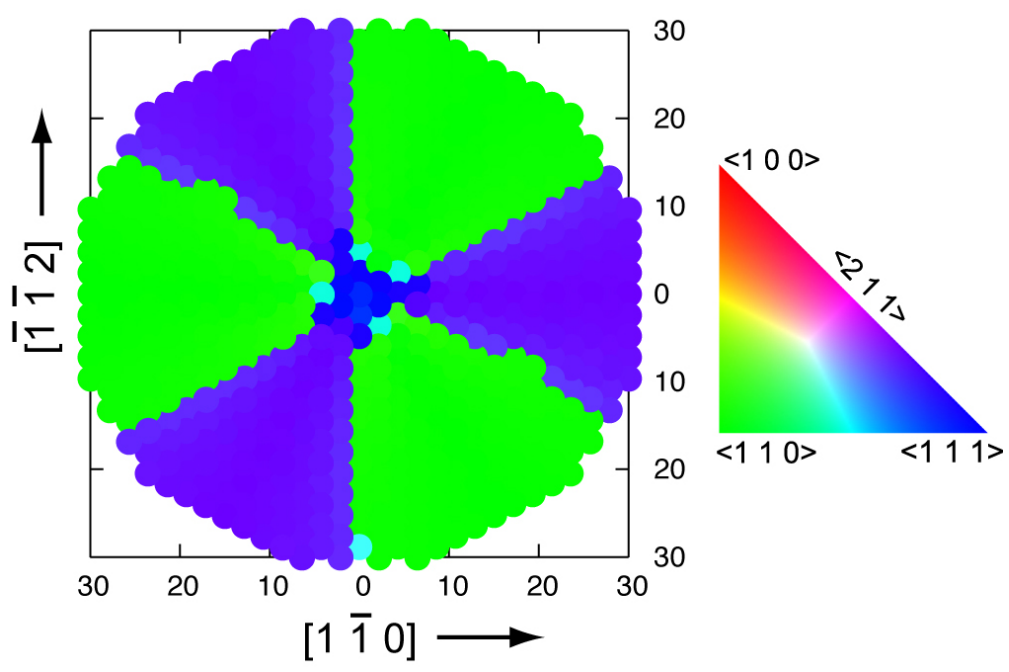

Figure 12: $\left[\begin{array}{lll}1 & 1 & 1\end{array}\right]$ dumbbells are placed at various angles and positions about the dislocation core and minimized; their final orientations are shown by color. Approximately one half end up as $<1 \quad 1 \quad 0>$-type; the other half remain strongly $<1 \quad 1 \quad 1>$ with some reorientation to align with the helical structure of the screw dislocation.

Where the dumbbells rotated only slightly, interaction energies remain strongly positive, while negative energies result when the dumbbell achieves the more stable configuration. The missing lattice sites near the core indicate where a dumbbell was absorbed in the dislocation, resulting in the low energy core structure.

Results from elasticity theory (Equation 9) are shown in Figure 14. Again, anisotropic theory reveals the non-degenerate core structure while isotropic theory neglects it. In fact, isotropic theory predicts almost no interaction at all. Because of the addition of the $E_{\text {defect }}^{<111>}=.48$ to the interaction energies, results from theory are referenced identically to those from atomistics, and the results can easily be compared. Where our atomistics show rotation to $<110>$ structures, the theory indicates interaction energies slightly below 0.48 for the $\left[\begin{array}{lll}1 & 1 & 1\end{array}\right]$. Dumbbells in these regions feel an attraction to the core which results in their reorientation to a more energetically favorable configuration. Thus, while anisotropic elasticity does not explicitly predict the spontaneous rotation of $<111>$ dumbbells, the preference for that rotation is present in the interaction energies of the $<111>$ dumbbell with the dislocation. Higher interaction energies are seen in the regions where no reorientation is observed atomistically. 


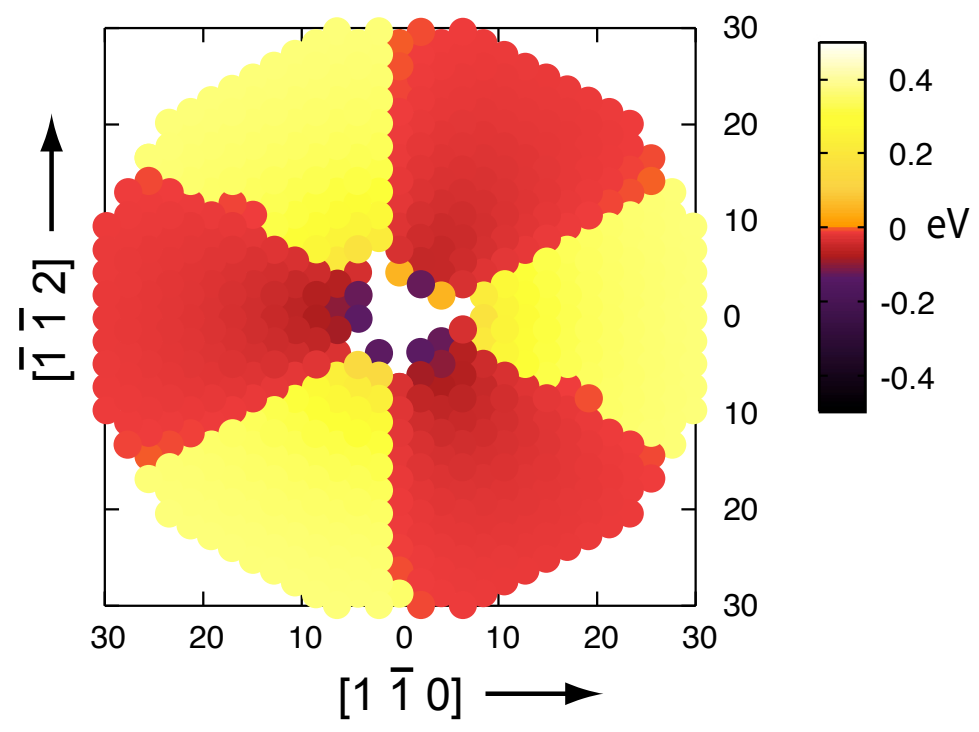

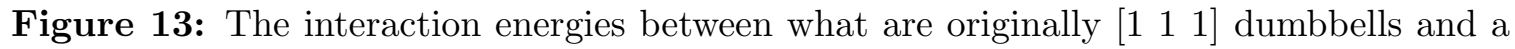
screw dislocation core are shown as a function of of distance and angle from the dislocation core. The same conventions as in Figure 9 are used. In the red regions, dumbbells convert to the more energetically favorable $<110>$-type.

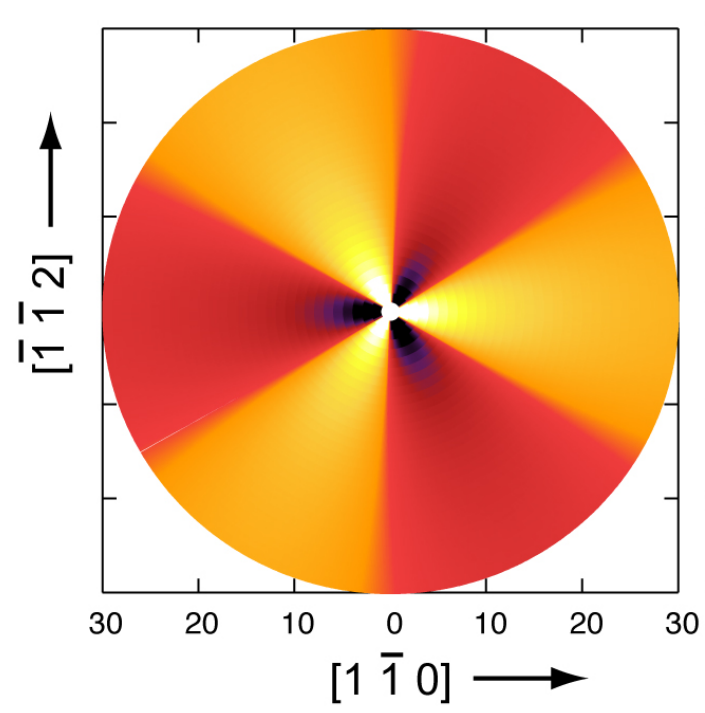

(a)

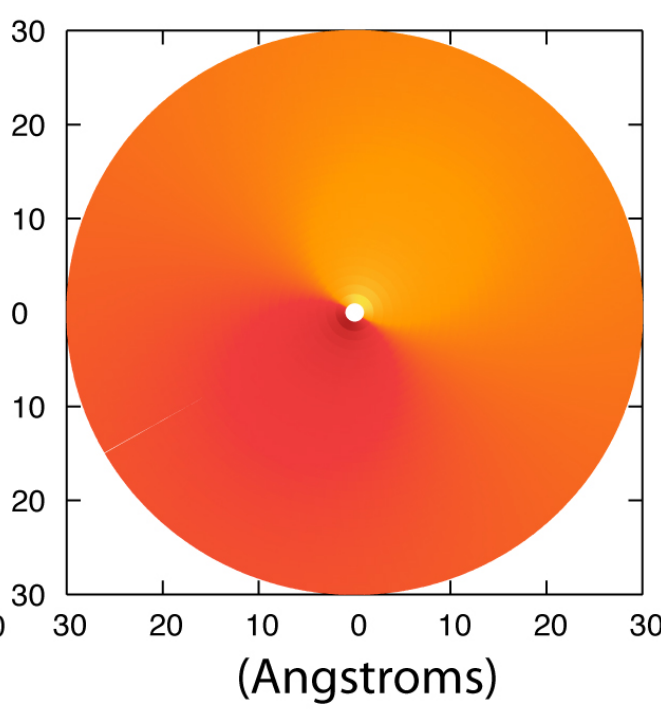

(b)

Figure 14: Interaction energies from elasticity theory (including the $E_{\text {defect }}$ term of Eq. 9) for a $\left[\begin{array}{lll}1 & 1 & 1\end{array}\right]$ dumbbell. Results using the anisotropic strain field are shown in (a); isotropic is shown in (b). Note that the energy is scaled differently than in previous plots, but quantitatively may be compared directly. 
Although anisotropic elasticity theory provides a glimpse into the energetics near the core, it is unable to predict where dumbbells may reorient and what their final state will be. That is, elasticity theory cannot determine when a given structure is stable, only what its energy is if one assumes that it is stable. Atomistics is needed to obtain this crucial information.

\subsection{3 $<100>$ Dumbbell}

The $\left[\begin{array}{lll}1 & 0 & 0\end{array}\right]$ dumbbell has the highest formation energy of the three primary dumbbells in the bulk and is generally not stable for any significant time. So, perhaps it is not surprising that this dumbbell is also unstable near a dislocation core. A complex landscape of energetics is revealed in Figure 15. As can be seen in Figure 16, this is due to the fact that all of the dumbbells originally introduced in the $\left[\begin{array}{lll}1 & 0 & 0\end{array}\right]$ orientation transform under minimization. Most become some type of $<110>$ or $<1 \overline{1} 0>$ structure. Which variant an individual dumbbell transitions to depends on its initial location about the core. Vectors are shown for each lattice site; these run along the resulting dumbbell, and many have partial extent into the page. $\left[\begin{array}{lll}1 & 0 & 0\end{array}\right]$ dumbbells lying near the $\left[\begin{array}{lll}1 & 0 & 1\end{array}\right]$ or $\left[\begin{array}{lll}1 & 1 & 0\end{array}\right]$ directions transition to be one of the indicated types, respectively; these are the lowest energy structures attained. In other areas, dumbbells become variants of $<\begin{array}{lll}1 & 0 & 0\end{array}$. In the $\left[\begin{array}{lll}0 & \overline{1} & 1\end{array}\right]$ direction, this configuration is not as low energy as a $\left[\begin{array}{lll}1 & 0 & \overline{1}\end{array}\right]$ shape would be. Presumably, the lowest energy $<\begin{array}{lll}\overline{1} & 0>\end{array}$ configurations are unattainable under a simple minimization, while a $<110>$ orientation provides an acceptable local minimum.

Due to symmetry considerations, the patterns of behavior described above will occur for the $\left[\begin{array}{lll}0 & 1 & 0\end{array}\right]$ and $\left[\begin{array}{lll}0 & 0 & 1\end{array}\right]$ dumbbell orientations, though rotated by $120^{\circ}$. Just as for the case of the $\left[\begin{array}{lll}1 & 1 & 1\end{array}\right]$ dumbbell, continuum elasticity would not be able to predict the unstable nature of the $\left[\begin{array}{lll}1 & 0 & 0\end{array}\right]$ dumbbell.

\subsubsection{Vacancy}

The results for vacant lattice sites are presented in Figure 17. Perhaps unsurprisingly, negative interaction energies are seen for vacancies in the regions where positive interaction energies are observed for interstitials, and vice versa. That is, where an interstitial is 


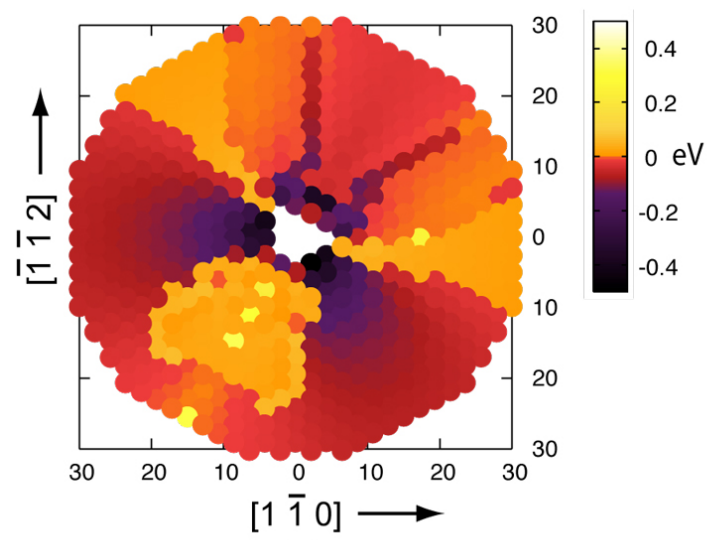

(a)

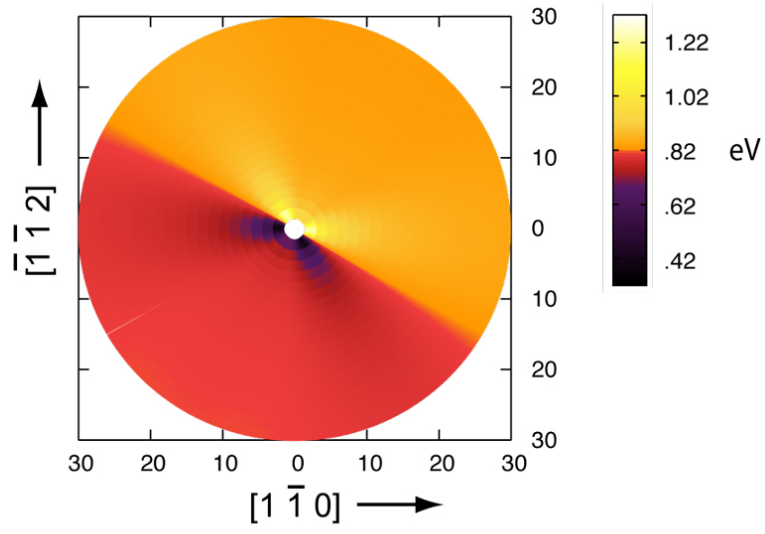

(b)

Figure 15: The interaction energies between [1 00 d dumbbells and a screw dislocation core are shown as a function of of distance and angle from the dislocation core. The same conventions as in Figure 9 are used. (a) shows the interaction energy calculated by atomistics. It is important to note that the majority of the dumbbells reorient to more energetically favorable configurations during minimization, so these energies do not truly represent the interaction between $\left[\begin{array}{lll}1 & 0 & 0\end{array}\right]$ dumbbells and the core. (b) shows $E$ calculated by anisotropic continuum theory; in this case the interaction energies are representative of $\left[\begin{array}{lll}1 & 0 & 0\end{array}\right]$ dumbbells. Note the different scales for the two graphs. Both are referenced for a value of 0 equal to a $<110>$ dumbbell in the bulk.

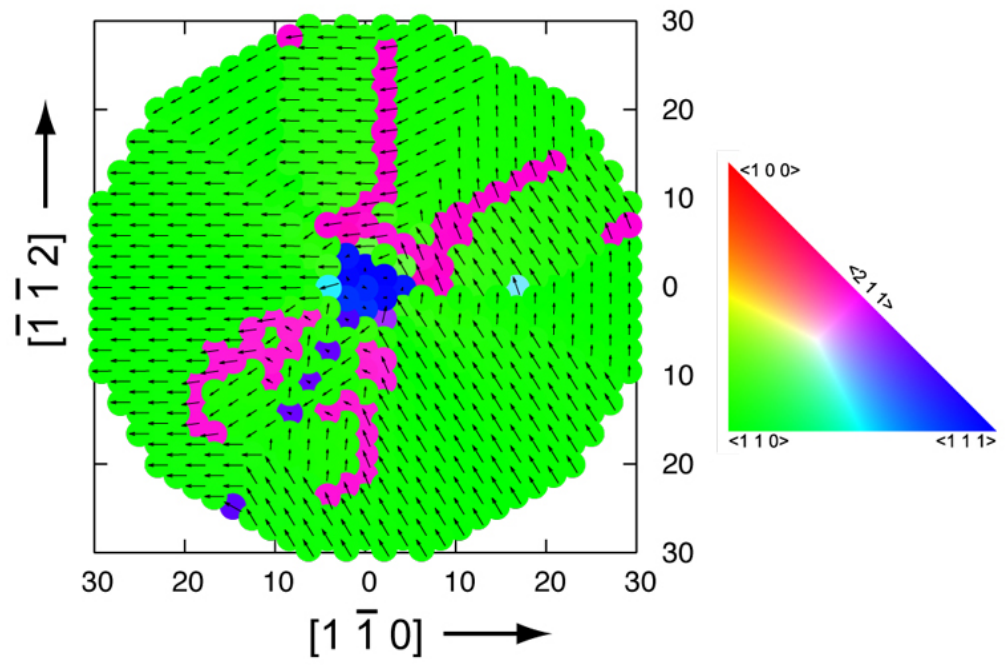

Figure 16: $\left[\begin{array}{lll}1 & 0 & 0\end{array}\right]$ dumbbells are placed at various angles and positions about the dislocation core and minimized; their final orientations are shown by color. The majority of dumbbells reorient to some variant of a $<110>$ dumbbell. Vectors on top of each point indicate the direction of the dumbbell (some are partially pointed into the page). 


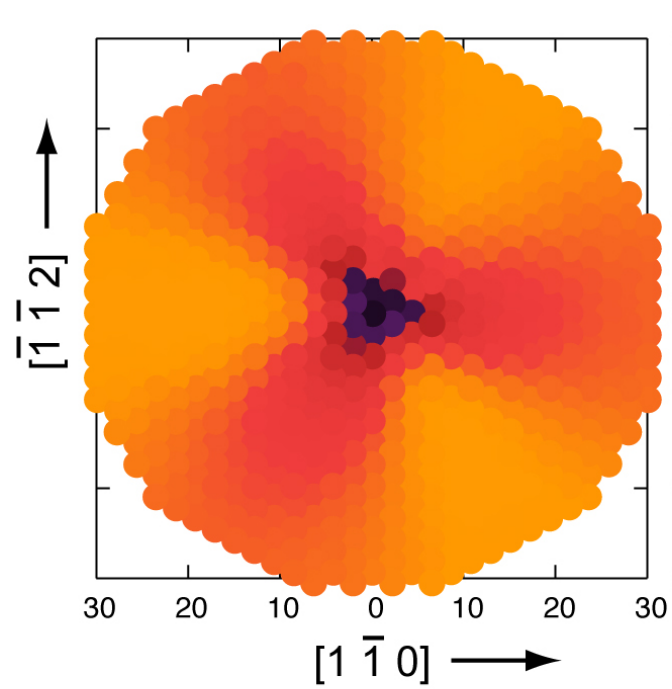

(a)

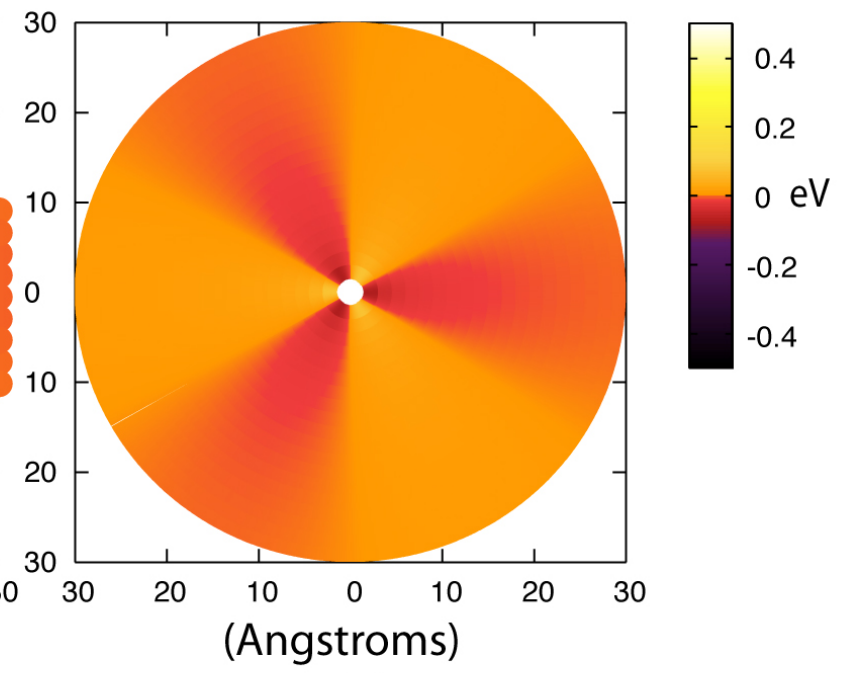

(b)

Figure 17: The interaction energies between vacancies and a screw dislocation core are shown as a function of of distance and angle from the dislocation core. The same conventions as in Figure 9 are used. Results from atomistic calculations are shown in (a); anisotropic continuum results are shown in (b). No results are displayed for isotropic elasticity theory; due to the nature of the matrices, this theory predicts no interaction whatsoever.

attracted to the dislocation, the vacancy is repelled, and vice versa. The magnitudes of the interactions for vacancies are significantly less than those for interstitials, however. Positive interaction energies do not exceed $0.003 \mathrm{eV}$, which indicates that the dislocation core never has a strong repulsive effect on vacancies. Negative interaction energies just outside the core dip to around $-0.07 \mathrm{eV}$; when a vacancy is absorbed by the core the system energy falls by about $0.35 \mathrm{eV}$. A vacancy has a symmetric dipole tensor which, unlike interstitial dumbbells, results in an isotropic distortion of the lattice around it.

When we compare results from atomistics to those of elasticity theory, agreement is quite good. The sign and magnitude of the interaction energy is similar everywhere except within about $10 \AA$ of the core. Here, atomistics shows a negative interaction energy in all directions, with absorption of the vacancy within about $5 \AA$ of the core. The behavior is qualitatively similar to that observed for the interstitial.

Comparison with isotropic elasticity theory is not shown because the vacancy dipole tensor has only diagonal components and the strain field has only off-diagonal components. As a consequence, no interaction is predicted by Equation 7 at all. 


\subsubsection{Absorption by the Core}

Spontaneous absorption of an interstitial defect by the core is observed, regardless of the initial orientation, when the defect is placed within a few angstroms of the core. The radius within which an interstitial will be absorbed is somewhat larger for the less stable defect configurations than for the $<110>$ types. Absorption of an interstitial results in a crowdion structure along the dislocation line. This crowdion may form along any of several of the columns of atoms nearest the core. These structures are essentially degenerate in energy when compared to the energy of the system before absorption. An example of this is shown in Figure 18. In Figures 12 and 16, the lattice sites which convert to this structure are shown in blue, with $<111>$ orientation. This absorption by the core generally occurs when the dumbbell is placed within an approximately triangular region about $6 \AA$ from the center of the dislocation; this volume encompasses all the lattice sites in any of 10 [ $\left.\begin{array}{lll}1 & 1 & 1\end{array}\right]$ columns parallel to the dislocation. In the case of $<1 \overline{1} 0>$ dumbbells, which are the most stable, absorption occurs only in the 5 columns closest to the core, as shown in Figure 9. This absorbed state is $\sim 2.5 \mathrm{eV}$ below the $0.0 \mathrm{eV}$ reference energy of the interstitial infinitely far from the dislocation; in other words, the system reduces its energy by about $2.5 \mathrm{eV}$ by absorbing an interstitial, or the binding energy of the interstitial to the core is about 2.5 $\mathrm{eV}$.

Similar to the interstitials, a triangular region of low energy configurations exists for the vacancy (see the purple region in Figure 17). Having a vacancy within this region lowers the system energy by about $0.35 \mathrm{eV}$ (the binding energy of a vacancy to the core is about $0.35 \mathrm{eV})$. However, unlike with absorption of an interstitial, the vacancy keeps its character within the core region. As is shown in Figure 19, the atoms near the vacancy relax around it, but the vacancy essentially remains localized to a lattice site.

\subsection{Discussion}

Absorption of interstitials in the dislocation core enables kink nucleation and glide, as well as core spreading $[33,117,118]$. These effects are modeled at longer length and time scales with large scale constitutive models, such as in [112], which rely on input from 

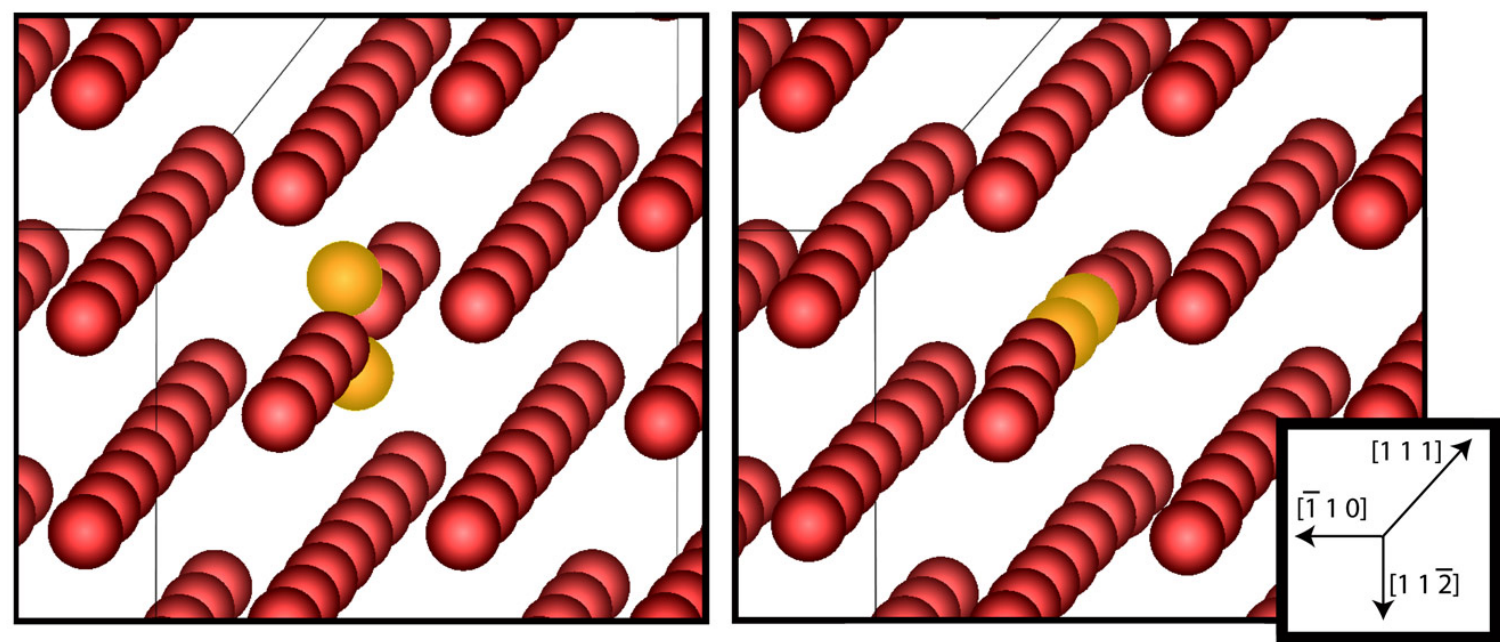

Figure 18: Core structure before (left) and after (right) minimization of a $\left[\begin{array}{lll}1 & 1 & 0\end{array}\right]$ dumbbell. The dumbbell reorients to become a crowdion along the dislocation line. The two atoms of the dumbbell are highlighted as a visual aid.
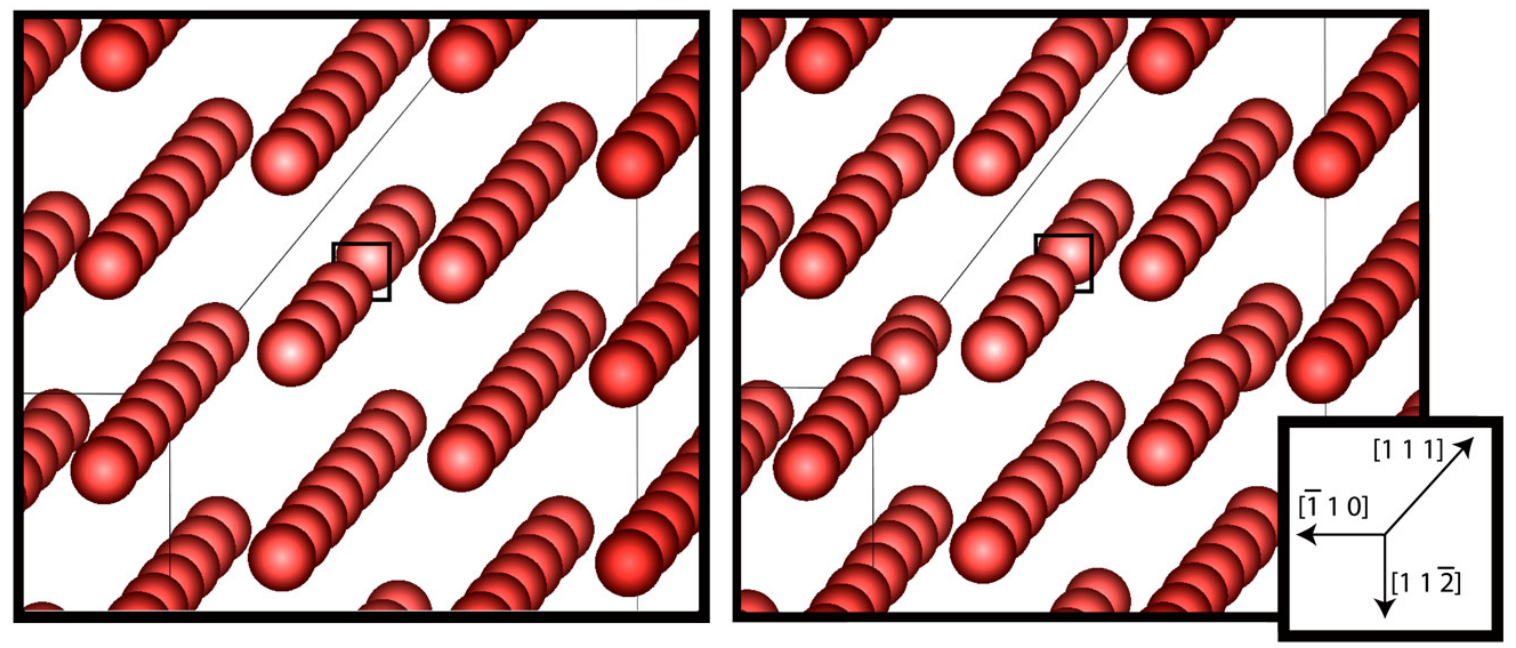

Figure 19: Core structure before (left) and after (right) minimization of a vacancy. The vacancy, marked by an empty box, maintains its character within the core; neighboring atoms relax around it. 


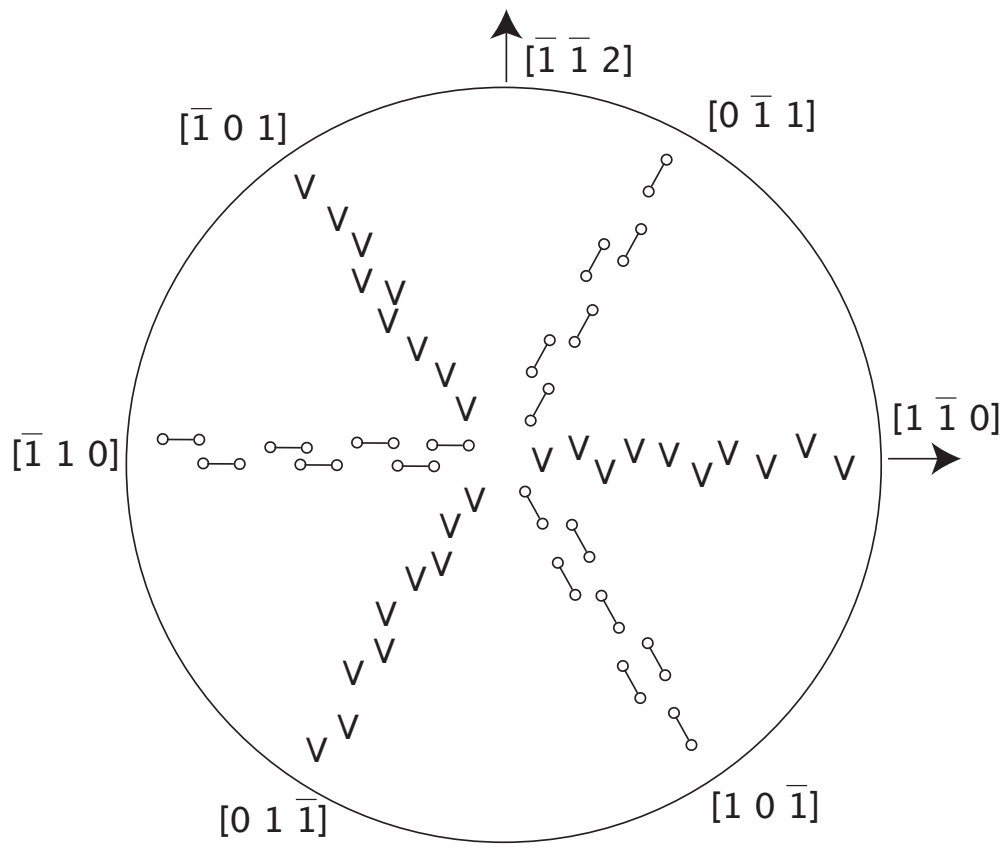

Figure 20: For a right-handed $1 / 2\left[\begin{array}{lll}1 & 1 & 1\end{array}\right]$ screw dislocation vacancies have the lowest interaction energies along the $\left[\begin{array}{lll}1 & \overline{1} & 0\end{array}\right],\left[\begin{array}{lll}1 & 0 & 1\end{array}\right]$, and $\left[\begin{array}{lll}0 & 1 & \overline{1}\end{array}\right]$ directions, while interstitials prefer to lie perpendicular to the dislocation line along the $\left[\begin{array}{lll}1 & 1 & 0\end{array}\right],\left[\begin{array}{lll}1 & 0 & \overline{1}\end{array}\right]$, and $\left[\begin{array}{lll}0 & \overline{1} & 1\end{array}\right]$ directions.

atomistic simulations to govern their dynamics. However, as was previously discussed, many of these codes still rely on isotropic formulations, because of the ease of implementation and generality.

Isotropic continuum theory is shown to be inadequate for describing the interactions between defects and the screw core in Fe based systems, both quantitatively and qualitatively. Anisotropic theory is certainly more promising, with overall good qualitative and improved quantitative agreement with atomistics; however, our results indicate that anisotropic elasticity calculations can not be used solely to describe the interactions between defects and dislocation cores. First, anisotropic calculations alone do not predict which dumbbell structures will be stable near the core. Second, quantitative agreement is not good in this region. These limitations do not preclude the use of anisotropic theory, but indicate that it must be carefully and thoughtfully applied.

In addition to being affected by displacements along the dislocation line, slip may be significantly affected by the atomic displacements perpendicular to the Burgers vector; these may be considered edge components of the displacement $[50,20,32,51]$. The stresses 
on these non-glide components affect the plastic behavior, modifying the critical resolved shear stress needed to move the dislocation. In the isotropic case, there is no displacement perpendicular to the dislocation line, only along it. In the anisotropic case, there are edge components of the displacement, however they are not as prominent as they are in the atomic model. This discrepancy is likely to be very important in determining the behavior of the interaction of the dislocation with defects very near the core.

Generically, the regions about the core can be split into those with a preference for vacancies (along the $\left[\begin{array}{lll}1 & \overline{1} & 0\end{array}\right],\left[\begin{array}{lll}\overline{1} & 0 & 1\end{array}\right]$, and $\left[\begin{array}{lll}0 & 1 & \overline{1}\end{array}\right]$ directions) and those with a preference for interstitials (along the $\left[\begin{array}{lll}\overline{1} & 1 & 0\end{array}\right],\left[\begin{array}{lll}1 & 0 & \overline{1}\end{array}\right]$, and $\left[\begin{array}{lll}0 & \overline{1} & 1\end{array}\right]$ directions), as shown in Figure 20. This segregation may reduce the overall recombination of defects in the vicinity of the dislocation, until absorption within the core itself. The determination of what orientation of interstitial dumbbell is preferred for a given lattice site is a problem for which atomistics

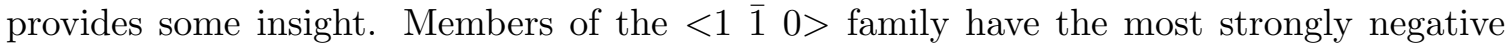
interaction energies, but only in the regions where having an interstitial is preferred over a vacancy. Here, a dumbbell pointed towards the core with no extent along the dislocation line is the lowest energy configuration. In the regions where $<1 \quad 1 \quad 0>$ dumbbells show positive interactions energies with the core, members of the $<1 \quad 10>^{+}$family are more likely than those from $<1 \overline{1} 0>$; both have positive interaction energies, but the former is less strongly repelled. [ [ $\left.\begin{array}{lll}1 & 1 & 1\end{array}\right]$ dumbbells may also survive in these regions, although they are slightly higher in energy. The $\left[\begin{array}{lll}1 & 0 & 0\end{array}\right]$ configuration is too unstable to exist near the core; it will typically reorient to a $<1 \quad 10>$ structure. Continuum theory does reveal the proper hierarchy of energetics, but does not show conclusively which orientations will be stable near the core, nor what shape an unstable dumbbell will reorient to under the core's influence. Knowing these details is critical for determining, for example, defect absorption rates into the dislocation core.

Near the core, continuum theory is completely unable to describe interactions, due to the $1 / r$ terms in the strain fields, consistent with previous work. Additionally, atomistics shows a negative interaction energy close to the core (within approximately $10 \AA$ ) for all defects in all direction, indicating the eventual absorption of the defect into the core. The core shows 
itself in atomistic simulations as a triangular region about the center of the dislocation with a radius of $\sim 6 \AA$. Continuum theory often shows positive interaction energies along particular directions, neglecting this important core effect. Far from the core, qualitative agreement is good for the $<110>$ dumbbells and the vacancies, where stability is not an issue (that is, there are minima in the potential energy landscape near all the initial positions of the defects). However, quantitative agreement is lacking. This issue could be improved with more advanced techniques for calculating the dipole tensor, such as including higher order terms beyond the first order. In any case, while anisotropic elastic theory does predict qualitative behavior far reasonably well, and could be used to form the basis of a higher level model of defect-dislocation interaction, the differences between elasticity and explicit atomistics are great enough that results should always be validated against atomistic calculations. 


\section{CHAPTER III}

\section{HYDROGEN BUBBLES}

\subsection{Motivation and Literature Review}

Understanding the mechanisms behind phenomena such as embrittlement, hardening, creep, and swelling requires a fundamental understanding of how these gases interact with defects in the microstructure. While it seems clear that hydrogen and helium do play an important role, there are many open questions on how exactly the gases assist in these processes $[107,24,96]$.

There is a large body of work on the role of hydrogen with steels. Many have documented the deleterious effects of hydrogen [107, 58, 18], but the processes responsible for the effects remain open for debate. In irradiated environments such as fission and fusion reactors and accelerator-driven systems, hydrogen may be present due to adsorption, implantation, or transmutation [45]. Hydrogen has a fairly low solubility in alpha-iron and a rapid diffusion rate; thus it can be difficult to study experimentally, especially at atomistic scales. Once in the bulk, hydrogen diffuses quickly between tetrahedral interstitial sites until reaching a surface or a trap, such as a vacancy or grain boundary [106]. Computational atomistic studies provide a chance to study the details of this process on a scale not possible with experiment $[87,72]$. There have been a large number of ab initio and atomistic scale studies performed on the iron-hydrogen system. These include studies on the diffusion of hydrogen through bulk iron $[120,66,139]$, the trapping of hydrogen at monovacancies $[62,115]$, and the accumulation of hydrogen at crack tips [131] and dislocations $[68,114,137]$.

Hydrogen embrittlement (HE) is a particularly important issue with many different theories about the cause behind it. Deformed, strained, and irradiated systems are particularly susceptible. There have been multiple theories proposed on what causes HE in metals [108, 109, 10, 147]. Hydrogen-enhanced localized plasticity (HELP) [10] theory predicts that hydrogen makes dislocations more mobile, leading to increased deformation in specific 
areas, and ultimately plastic failure. Hydrogen may also cluster at crack tips, eventually leading to decohesion [108].

However, there is recent evidence that vacancy assisted mechanisms may play the most important role in HE $[140,101,63]$. The excessive numbers of vacancies generated by irradiation may be stabilized internally by hydrogen, allowing for the growth of small individual vacancies into large voids. The resulting increase in plasticity leads to failure of the steel $[101,102]$. A thorough understanding of the thermodynamics of vacancy-hydrogen clusters is essential to determining the role that hydrogen bubbles may play in the extremely complex picture of hydrogen embrittlement.

Experimental results from ion beam implantation and detrapping studies provide a basis for comparison and verification of atomistic studies. Ion-beam experiments by Myers et al. [98] investigated defect trapping of deuterium in iron. Using ion-channeling analysis, the locations of hydrogen atoms near defects can be deduced when measurements are taken along at least two axes. Deuterium atoms were implanted in an bcc iron single crystal and the crystal was annealed to allow the D to move to traps. Analysis showed that a D trapped at a monovacancy resides in a positions offset by $\delta=0.4 \AA$ from an octahedral interstitial site (O-site) in the direction of the vacancy. The authors reported that their results allow for alternate interpretations involving multiple lattice positions for $\mathrm{D}$, but did not feel that this more complex explanation was necessary. Two detrapping stages were identified at $260 \mathrm{~K}$ and 350-450 $\mathrm{K}$ when the temperature of the samples was ramped up, with binding energies of 0.48 and $0.81 \mathrm{eV}$, respectively. The first release was associated with $\mathrm{D}$ trapped at monovacancies, while the higher temperature release was speculated to correspond to vacancy clusters or dislocation trapping. Effective medium theory (EMT) was proposed to explain these experimental results [104, 103].

Later work by Myers et al. [97] identified three types of defect traps, with energies of 0.53, 0.71, and $0.78 \mathrm{eV}$, when iron was implanted with both $\mathrm{D}$ and He. The first type was assumed to be due to monovacancies, the second type due to vacancy clusters, and the third type is due to trapping at He bubbles. Further work was performed by Besenbacher et al. [8]. When the fluence was increased compared to the above mentioned experiment, an 
additional release stage developed at $220 \mathrm{~K}$. The authors deduced that this stage was due to multiple occupancy of a vacancy by up to six D atoms. When accounting for vacancy mobility, it was found that 1-2 D are trapped by a vacancy with strength of $0.63 \mathrm{eV}$, while 3-6 D are trapped by $0.43 \mathrm{eV}$. A review of many of these results, as well as additional results for surface interactions and a transport formalism for hydrogen behavior, are described in a review paper by Myers et al. [99]. Results from effective-medium theory (EMT) [104, 103] reasonably well to these experimental results, with 1-2 D trapping of about $0.8 \mathrm{eV}, 3-4 \mathrm{D}$ trapping around $0.52 \mathrm{eV}$, and 5-6 D trapping around $0.41 \mathrm{eV}$. These numbers were generated by assuming that all D occupied an offset O-site, with symmetric minimization allowed in the direction of the nearest $\left(\begin{array}{lll}1 & 0 & 0\end{array}\right)$ planes.

One of the most crucial pieces of information that must be known about hydrogenvacancy systems if complex studies are to be performed is the basic energetic information. Additional experimental results and most recent density functional theory calculations seek to answer this question by determining how many hydrogen atoms may be bound to a monovacancy. A hydrogen atom can be considered to be exothermically bound to a vacancy its binding energy exceeds the heat of solution. The heat of solution of hydrogen in iron is quoted as slightly different values from different sources, including $0.25 \mathrm{eV} /$ atom [40], 0.29 $\mathrm{eV}[81]$ and $0.32 \mathrm{eV} /$ atom [141] from experiment and calculation. Since experiment showed that up to six hydrogen atoms are trapped by $0.43 \mathrm{eV}$, larger than any of the found heats of solution, it was generally agreed upon in the literature that all six offset O-sites around a monovacancy are exothermic $[63,40]$, and the $\mathrm{H}_{6} \mathrm{~V}$ complex will be dominant over the $\mathrm{H}_{2} \mathrm{~V}$ complex in the bulk.

Tateyama and Ohno [141] performed density functional theory (DFT) calculations that disagreed with this view. In a 54 atom supercell, up to six hydrogen atoms were placed in offset O-sites. The first two hydrogens were bound by $\sim 0.60 \mathrm{eV}$, in good agreement with the value of $0.63 \mathrm{eV}$ from experiment. For 3-5 hydrogen atoms, trapping energies ranged between about 0.4 and $0.3 \mathrm{eV}$, also showing agreement with the experimental $0.43 \mathrm{eV}$. The sixth hydrogen atom showed a slightly negative binding energy, indicating that it would not be trapped. Compared to the hydrogen heat of solution, which the authors calculated 
to be $0.32 \mathrm{eV}$, only 1-3 hydrogen atoms were exothermic, with the third just barely so. The authors concluded that the $\mathrm{H}_{2} \mathrm{~V}$ state is actually dominant, not the conventionally accepted $\mathrm{H}_{6} \mathrm{~V}$ state. Based on these results, the authors investigate the shapes of $\mathrm{H}_{2} \mathrm{~V}$ clusters, finding that anisotropic clusters with linear and tabular shapes will be generated.

While there exist many studies on hydrogen bubbles, a fundamental understanding of themechanisms behind their effects is still largely lacking. Using empirical potentials with molecular dynamics techniques, we are able to model much larger systems than are currently possible with first principles techniques, while retaining the detail of modelling individual atoms. In this chapter, we simulate clusters of hydrogen and vacancies within alpha-iron to determine their energetic properties.

\subsection{Methods}

Our goal is to simulate hydrogen-vacancy clusters and calculate their minimum energy configurations. Clusters consisting of $m$ hydrogen atoms and $n$ vacancies $\left(\mathrm{H}_{m} \mathrm{~V}_{n}\right)$ are created in bcc iron. Unless otherwise mentioned, a simulation cell of size $15 a_{0} \times 15 a_{0} \times 15 a_{0}$ is employed, where $a_{0}=2.8553$ is the lattice constant of iron. In a perfect cell, there are 6750 Fe atoms. Periodic boundary conditions are used in all dimensions. The LAMMPS Molecular Dynamics Simulator [113] is used for all relaxation steps.

For the interatomic potentials, two variants of an embedded atom method (EAM) potential developed by Ramasubramaniam et al. [119, 121] for iron and hydrogen are used for all calculations. Referred to by the developers as potentials B and $\mathrm{B}^{\prime}$, these potentials both take their Fe-Fe interactions from Ackland et al. [3], while the $\mathrm{H}-\mathrm{H}$ and $\mathrm{Fe}-\mathrm{H}$ interactions are fit from density functional theory (DFT) data. Potential $\mathrm{B}^{\prime}$ is fit to bulk and vacancy DFT data, while potential B is additionally fit to surface DFT data. Potential B is recommended over $\mathrm{B}^{\prime}$ by the potentials' authors, however they note that $\mathrm{B}^{\prime}$ performs slightly better under a strain field in a bulk environment. Since our system is essentially a bulk environment lacking surfaces, it was unclear which variant would be more well-suited to the problem of hydrogen-vacancy clusters. We test both potentials and characterize the thermodynamic properties of the resulting cluster configurations by calculating formation energies 
and binding energies of vacancies, hydrogen atoms, and self-interstitial atoms (SIAs).

A formation energy is defined as the difference between the total energy of the system of interest and the sum of the energies of the constituent parts when separated. Thus, the formation energy of each $\mathrm{H}_{m} \mathrm{~V}_{n}$ bubble is calculated by

$$
E_{f}\left(\mathrm{H}_{m} \mathrm{~V}_{n}\right)=E_{t o t}\left(\mathrm{H}_{m} \mathrm{~V}_{n}\right)-\left\{(N-n) E_{c o h}^{F e}+m E_{c o h}^{H}\right\}
$$

where $N$ is the total number of atoms in the perfect system, the tot subscript indicates total system energy and the coh subscript indicates cohesive energy. Cohesive energy is calculated for bcc iron at $E_{c o h}^{F e}=-4.013 \mathrm{eV} /$ atom, and the cohesive energy for hydrogen is taken to be $E_{c o h}^{H}=-2.37 \mathrm{eV} /$ atom [119]. Additionally, formation energies are calculated for $<110>$ SIAs, giving a value of $E_{f}^{S I A(110)}=3.529 \mathrm{eV}$, and for hydrogen interstitials occupying the tetrahedral interstitial site, with $E_{f}^{H}=0.292 \mathrm{eV}$ or $0.296 \mathrm{eV}$ for potentials B and $\mathrm{B}^{\prime}$, respectively. $E_{f}^{H}$ is equivalently termed the heat of solution of hydrogen in iron. In order for the formation energies calculated to be meaningful, it is imperative to use minimum system energies. However, it is non-trivial to find the minimum energy configurations of $(m+n)$-body systems; we use a combination of molecular dynamics and Monte Carlo (MC) algorithms to search for these configurations.

First, the energetics of voids without hydrogen are investigated. A vacancy is introduced into the simulation cell, and the system is minimized using a conjugate gradient algorithm, yielding a single vacancy formation energy $E_{f}^{V}$ of $1.721 \mathrm{eV}$. Next, the atom with the highest potential energy is removed from the system, and again the system is minimized. This scheme is iteratively conducted to create voids up to 10 vacancies, and the formation energy of each is calculated. These systems with $n$ vacancies form the initial configurations into which hydrogen atoms are later introduced. In practice, the voids are roughly spherical.

Next, hydrogen atoms are introduced to the systems of $n$ vacancies. For this initial study, each combination of $m$ and $n$ that fit the following conditions is considered: $0<m<50$, $1<n<10$, and the ratio $(m / n) \leq 10$.

A region of radius $1.3 \AA$ is defined around each of the $n$ vacant lattice sites. $m$ hydrogen atoms are randomly inserted into the volume formed by the union of these regions. The 
system is minimized to a local minimum using conjugate gradient in the LAMMPS MD Simulator. Then, the system is quenched to $0 \mathrm{~K}$ by scaling down the velocity components of each atom. The total system energy is measured and recorded. At this point, a Metropolis Monte Carlo scheme [85] is used, in combination with minimizations through molecular dynamics, to aid in finding lower energy configurations. Every hydrogen in the system is randomly displaced from its site by a maximum of $r_{\max } \AA$ in each of the $x, y$, and $z$ directions. The system, including both the iron and hydrogen atoms, is again minimized at $300 \mathrm{~K}$ with the LAMMPS MD Simulator to come to a new local minimum, then quenched to $0 \mathrm{~K}$. If the total system energy is lower than in the previous state $(\Delta E<0)$, the transition is accepted, and the newly found configuration is used as the basis for the next iteration. If $\Delta E>0$, the transition is accepted with probability $p=\exp (-\Delta E / k T)$, where $k$ is Boltzmann's constant and $T$ is absolute temperature. Accepting some transitions that raise the system energy allows the cluster to avoid getting stuck in local minima. Iterations are repeated until the stopping criterion, discussed below, is met. An iteration consists of three steps: moving the hydrogen atoms within the cluster, minimizing the entire system using MD methods, and comparing the energy to previously seen states and accepting or rejecting the new state with MC criterion. A schematic of this iterative process is shown in Figure 21.

The systems tend to find lower energy states infrequently, but with relatively large decreases in energy, instead of finding slightly lower energy states on nearly every step. Thus, we use a stopping criterion based on number of iterations, instead of stopping when the change in energy is below some set value. Each bubble simulation is continued for a minimum of 1000 steps, and the total number of steps is adaptively increased for each case so that the simulation runs for at least $q$ steps without finding a new lowest energy state.

Additionally, a subset of clusters $\left(\mathrm{H}_{m} \mathrm{~V}_{1}, 1 \leq n \leq 6\right)$ are replicated in a smaller $3 a_{0} \times 3 a_{0} \times 3 a_{0}$ simulation cell, using only potential B. Density functional theory (DFT) is also used to study the energetics of this subset of configurations. In these calculations, performed by Benjamin Beeler, the lowest energy configuration for each $\mathrm{H}_{m} \mathrm{~V}$ system is 


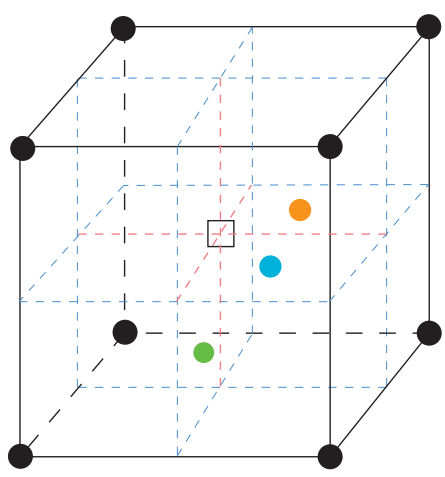

(a)

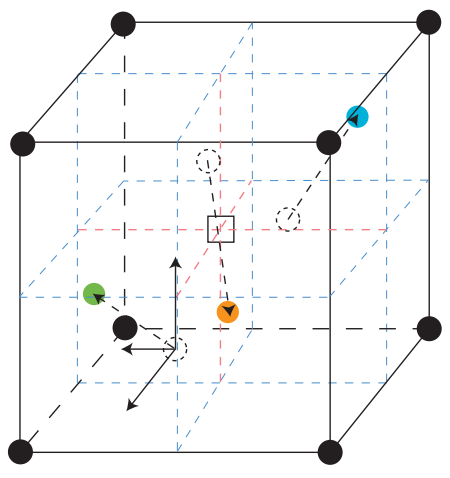

(c)

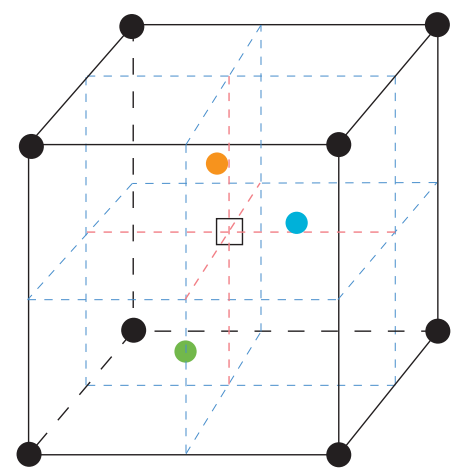

(b)

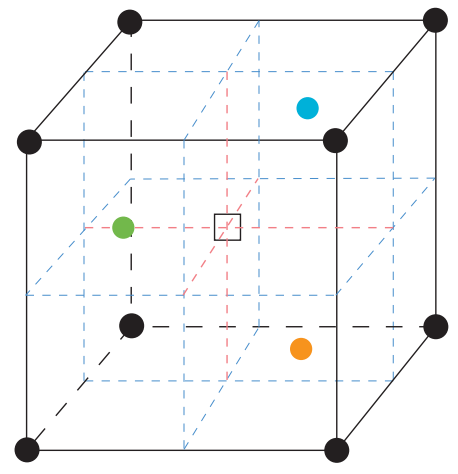

(d)

Figure 21: The Monte Carlo process is depicted schematically for the $\mathrm{H}_{3} \mathrm{~V}_{1}$ cluster. First, atoms are randomly inserted (a), then minimized (b). The atoms are displaced randomly in the $\mathrm{x}, \mathrm{y}$, and $\mathrm{z}$ directions (c) and minimized multiple times. Finally, the low energy configuration is found (d). Hydrogen atoms are shown colored, the vacancy is shown as a square in the center of a bcc iron cell. 
placed into a 54 atom supercell and analyzed with the Vienna ab initio Simulation Package (VASP) [71, 67, 11]. The Projector Augmented Wave (PAW) method [65] is utilized within the density functional theory $[61,70]$ framework. Calculations are performed using the Perdew-Burke-Ernzerhof (PBE) [14] Generalized Gradient Approximation density functional implementation for the description of the exchange-correlation. Methfessel and Paxton's smearing method [84] of the first order is used with a width of $0.2 \mathrm{eV}$ to determine the partial occupancies for each wave function. Relaxations are performed using the conjugate gradient method with a convergence criterion on the forces of $0.02 \mathrm{eV} / \AA$. An iron PAW pseudopotential with the $3 \mathrm{~d}^{7} 4 \mathrm{~s}^{1}$ valence electronic configuration and a core represented by $[\mathrm{Ar}]$ along with a hydrogen PAW pseudopotential with a $1 \mathrm{~s}^{1}$ valence electronic configuration is utilized. A cell was analyzed removing symmetry restrictions with a $4 \mathrm{x} 4 \mathrm{x} 4$ gamma-centered k-point mesh, resulting in 36 irreducible k-points in the Brillouin zone. To make the calculations more accurate, the energy cutoff was increased to $500 \mathrm{eV}$.

\subsection{Results}

\subsubsection{Performance of Interatomic Potentials}

It is desirable to find the ground state, or lowest energy, configuration of hydrogen atoms with a void of a given size in order to accurately calculate its energetics. Since the formation and binding energies are on the order of 1-10 eV, configurations with higher energies will cause scatter in the data. Optimizing the iterative scheme to search out very low energy configurations with a reasonable amount of computational resources is needed.

The three parameters that control the speed and accuracy of the scheme are $r_{\max }, q$, and T. $r_{\max }$ controls the maximum distance in each of the $x, y$, and $z$ directions that hydrogen atoms are displaced each iteration, $q$ determines the number of iterations that will be run, and $T$ gives the temperature that is considered when accepting or rejecting transitions.

To find the optimal values for each of these parameters for each potential, a test system with $m=18$ and $n=3$ is considered for its relatively small size but high cluster density [54]. The two potentials vary considerably in their performance. For each parameter variant, two trials were run, resulting in a total of 34 trials for each potential. When the same energy 
is recovered multiple times, we may be fairly certain that this is the lowest energy state of the system. This is the case for potential $\mathrm{B}^{\prime}$, where $29 / 34$ of the trials found the same lowest energy configuration. However, energies varied tremendously for potential B. The lowest energy that was recovered was seen in only one case, with the average system energy over all trials being $0.25 \mathrm{eV}$ higher than the minimum. This difference is significant in the calculation of bubble formation and binding energies, which are on the order of $1 \mathrm{eV}$.

Values of $r_{\text {max }}$ between 0.4 and $1.2 \AA$ in increments of $0.1 \AA$ were tested. Values between 0.7 and 1.0 were found to be suitable. Smaller values do not allow for a thorough search of the potential energy surface (PES), and the hydrogen atoms fall back into their previous positions under minimization. Larger values may cause hydrogen to be artificially removed from the bubble or may cause too much randomness, cancelling out the benefits of using MC. For both potentials, increasing $r_{\max }$ results in increased time per iteration. One value of $r_{\max }$ must be used for final calculations over all possible combinations of $m$ and $n$, since the calculated binding energies for a $\mathrm{H}_{m} \mathrm{~V}_{n}$ bubble depend on the formation and system energies of bubbles possessing $m \pm 1$ and $n \pm 1$ atoms. A value of $r_{\max }=1.0 \AA$, nearly equal to twice the Bohr radius, was selected such that approximately $1 / 10$ of the proposed transitions that raise the energy will be accepted. This accounts for thermal motion over a wide variety of combinations of $m$ and $n$.

Changing the value of $T$ used for the Monte Carlo step affects the number of energy raising configurations that will be accepted. For the same reasons as were described for $r_{\max }$, very low or high values are inappropriate. We tested $T=200,250,300,350,400$, 450, and $1000 \mathrm{~K}$, and found that values between 300 and 450 gave good results. $T=300$ was used for the results presented in this paper.

The value of $q$ determines how many iterations will be run before the system settles on a final energy; values of 500, 1000, 1500, and 2000 were tested. Increasing $q$ significantly increases computational time, with each iteration taking approximately 1 minute on one processor. For potential B, trials with values of $q$ greater than 500 were more likely to result in lower final energies. However, in all cases the number of iterations between lowest energy states was less than 1000 , so $q=1000$ was chosen for all runs using potential B. $q=500$ 


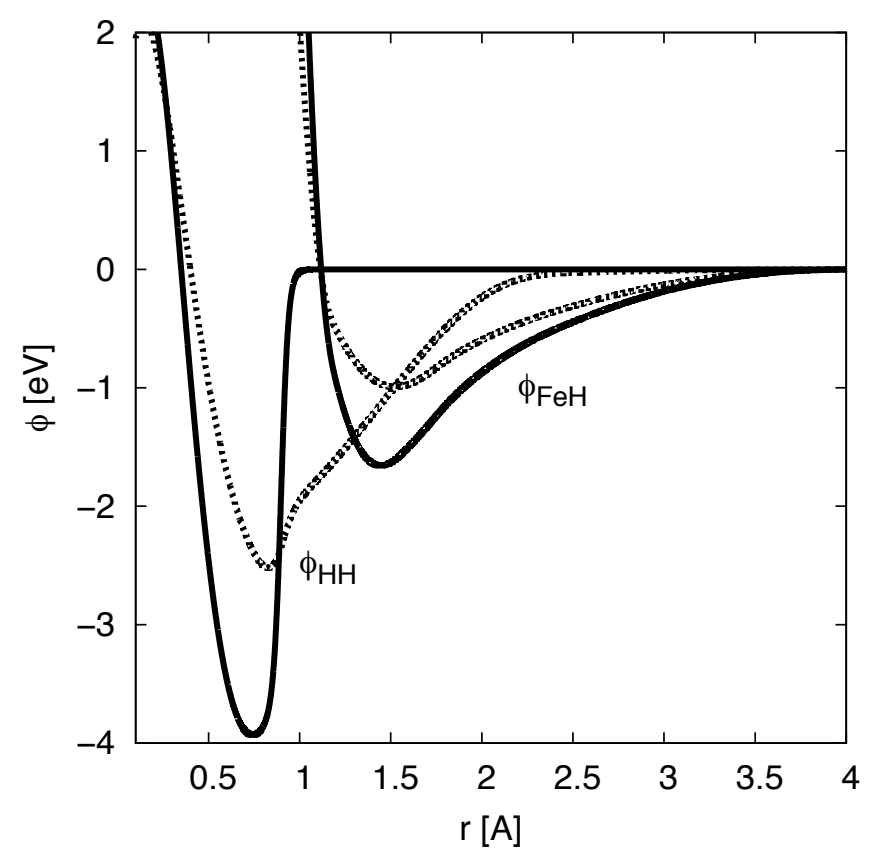

Figure 22: The pair potential part of the EAM potential is shown for $\mathrm{Fe}-\mathrm{H}$ and $\mathrm{H}-\mathrm{H}$ interactions. Solid lines indicate potential B, dotted lines indicate potential $\mathrm{B}^{\prime} . \phi_{F e F e}$ is identical for both potentials.

was found to be sufficient to minimize systems using potential $\mathrm{B}^{\prime}$.

A smaller battery of tests were also run on the lower ratio $\mathrm{H}_{24} \mathrm{~V}_{8}$ system to confirm the results. Results were consistent with those described above.

The potential energy surfaces generated by potentials $\mathrm{B}$ and $\mathrm{B}^{\prime}$ are very different and so result in different minimization characteristics. Both potentials are of the EAM type, with contributions to the total energy coming from pair potential and electron embedding functions. The pair potential functions for the $\mathrm{H}-\mathrm{H}$ and $\mathrm{Fe}-\mathrm{H}$ interactions for both potentials can be seen in Figure 22. Due to the deep, short-ranged H-H interaction of potential B, the PES is complex, pitted, and difficult to fully explore in a reasonable amount of time. The gradual cutoff of the $\mathrm{H}-\mathrm{H}$ interaction with potential $\mathrm{B}^{\prime}$ results in a much smoother PES. Overall, potential $\mathrm{B}^{\prime}$ is much quicker and easier to minimize with confidence than potential B. 

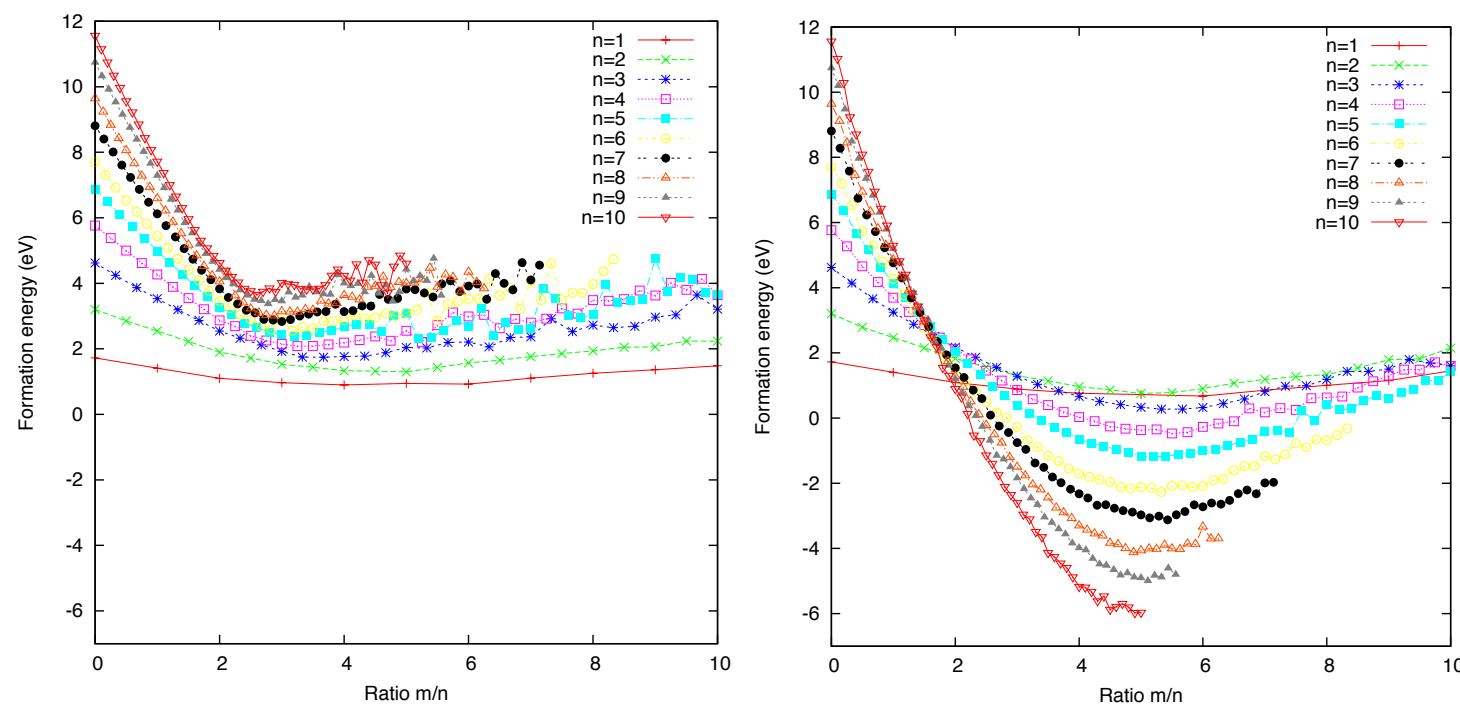

Figure 23: Formation energy curves for clusters of $m$ hydrogen atoms and $n$ vacancies. Results for potential $\mathrm{B}$ are on the left and potential $\mathrm{B}^{\prime}$ on the right. Ratio $m / n$ is shown on the abscissa, formation energy on the ordinate, while curves indicate different values of $n$.

\subsubsection{Energetics}

The formation energy, calculated using Equation 19, for each configuration can be seen in

Figure 23. Important theoretical differences between the two potential variants become apparent when comparing these two graphs. For potential B, the formation energy of a bubble increases as $n$ increases and is never negative. The minimum formation energy for a particular value of $n$ shifts from occurring at $m / n=5$ for $n=1$ to $m / n=2.6$ for $n=10$. In contrast, the formation energy of a bubble calculated with $\mathrm{B}^{\prime}$ decreases as $n$ increases, becoming more strongly negative as the void grows in size. The minimum formation energy for a given $n$ always occurs at $m / n=5$. The trend seen with potential $\mathrm{B}^{\prime}$ suggests that it is energetically favorable for hydrogen to be inside a bubble within bulk iron instead of taking molecular form outside of the bulk. However, iron is not observed to absorb large quantities of hydrogen; in fact, the opposite is true - hydrogen has a very low solubility in iron. The trend shown by potential B is more physically believable. It is energetically preferable for hydrogen to be outside of iron than trapped in a void, but the formation energy of the cluster is low enough that a hydrogen-vacancy cluster may form and survive in the right conditions. 
The jaggedness seen in the formation energy curves is a product of two effects. First, our method is non-exhaustive in searching for low energy configurations unless an infinite amount of time is allowed for the search, and the global minimum may not be found for every cluster. This is occasionally the case for high $n$ or $m / n$ clusters. Second, when the ratio is too high to support further hydrogen binding, a cluster may eject some hydrogen atoms out of the bubble proper, effectively resulting in a bubble with a lower ratio. This appears as scatter in the data, but is actually an important indicator of how much hydrogen a bubble may support. Potential B suffers compared to potential $\mathrm{B}^{\prime}$ in both these regards, due to the potential shape and characteristics.

The energetics of a cluster of given composition were characterized by the binding energies of a vacancy, a hydrogen atom, and an SIA to that cluster. The following equations were used for the calculations:

$$
\begin{gathered}
E_{B}(V)=E_{f}\left(\mathrm{H}_{m} \mathrm{~V}_{n-1}\right)+E_{f}^{V}-E_{f}\left(\mathrm{H}_{m} \mathrm{~V}_{n}\right) \\
E_{B}(H)=E_{f}\left(\mathrm{H}_{m-1} \mathrm{~V}_{n}\right)+E_{f}^{H}-E_{f}\left(\mathrm{H}_{m} \mathrm{~V}_{n}\right) \\
E_{B}(S I A)=E_{f}\left(\mathrm{H}_{m} \mathrm{~V}_{n+1}\right)+E_{f}^{S I A}-E_{f}\left(\mathrm{H}_{m} \mathrm{~V}_{n}\right) .
\end{gathered}
$$

These equations are similar to those used by Morishita et al. [92] in their work on the thermal stability of helium-vacancy clusters in iron. Like for their bubbles, we find that the trends in binding energies can be most easily described as functions of the ratio of $m$ to $n$.

As can be seen in Figure 24, the presence of hydrogen strongly affects the binding of vacancies to voids. Both potentials show that the binding energy of a vacancy to a cluster increases as the hydrogen inventory increases, indicating that hydrogen has a stabilizing effect on a bubble. With potential $\mathrm{B}$, the data become scattered as the ratio approaches 4, while the data for potential $\mathrm{B}^{\prime}$ does not show scatter until a ratio of near 7 . This is an indication of the highest ratio that is supported by each potential. Bubbles with ratios higher than 4 and 7 for potentials B and $\mathrm{B}^{\prime}$, respectively, have emitted hydrogen atoms from the cluster core, giving them effectively lower ratios and throwing off the binding energy calculations. For both potentials, the 'even' clusters with $n=6,8$, and 10 vacancies have the higher values of $E_{B}(V)$ than do the 'odd' clusters of $n=5,7$, and 9 vacancies. The 

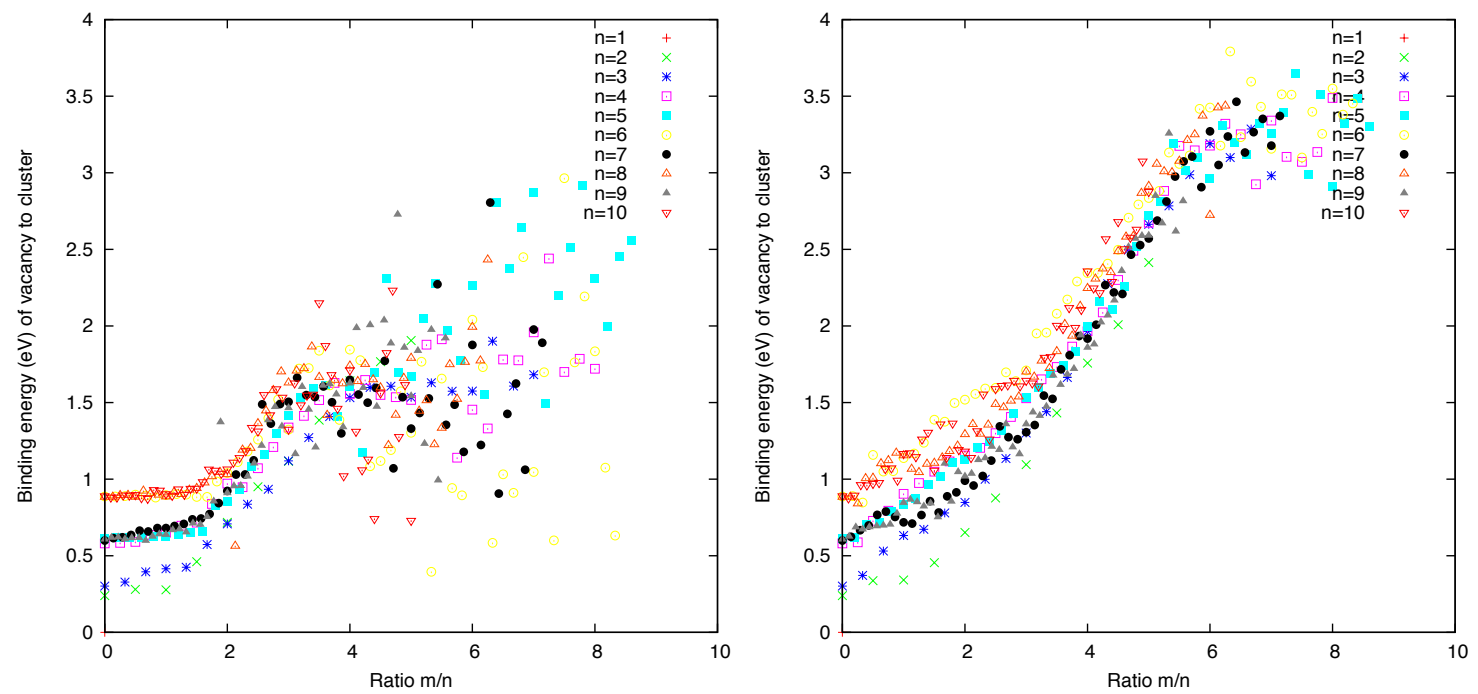

Figure 24: Binding energy of a vacancy to the cluster for potentials B (left) and B' (right). Ratio $m / n$ is shown on the abscissa, binding energy on the ordinate, while curves indicate different values of $n$. As hydrogen inventory increases, so does the binding of vacancies.

odd voids lack the symmetry of the even voids, making them less tightly bound, whether or not hydrogen is present.

The opposite trend is observed in the binding of iron atoms neighboring the cluster (Figure 25). These neighbors may be emitted as self-interstitials into the bulk if not tightly bound, effectively increasing the size of the bubble (i.e. loop punching or Frenkel pair production). Fe atoms are more easily removed from their lattice sites as the density of hydrogen is increased, leading to an effective increase in bubble size. This is another indication of hydrogen's stabilizing effect on vacancy clusters. With potential B, we observe a slow decrease in binding energy from ratio 0 to 2 , then a more dramatic increase until scattering begins at around $m / n=4$. Potential $\mathrm{B}^{\prime}$ displays a gradual decrease in SIA binding energies until leveling off and beginning to scatter as the ratio approaches 7 and bubbles eject hydrogen atoms to maintain low ratios. Again, we observe some splitting in the curves due to evenness or oddness of the void.

Finally, Figure 26 shows the binding energy of a hydrogen atom to the cluster vs the ratio of hydrogen atoms to vacancies. Like with SIAs, this number decreases as ratio increases. A hydrogen atom can be considered to be unbound from the bubble if its binding energy is less than the heat of solution $(0.29 \mathrm{eV})$. Each curve crosses this line at approximately 

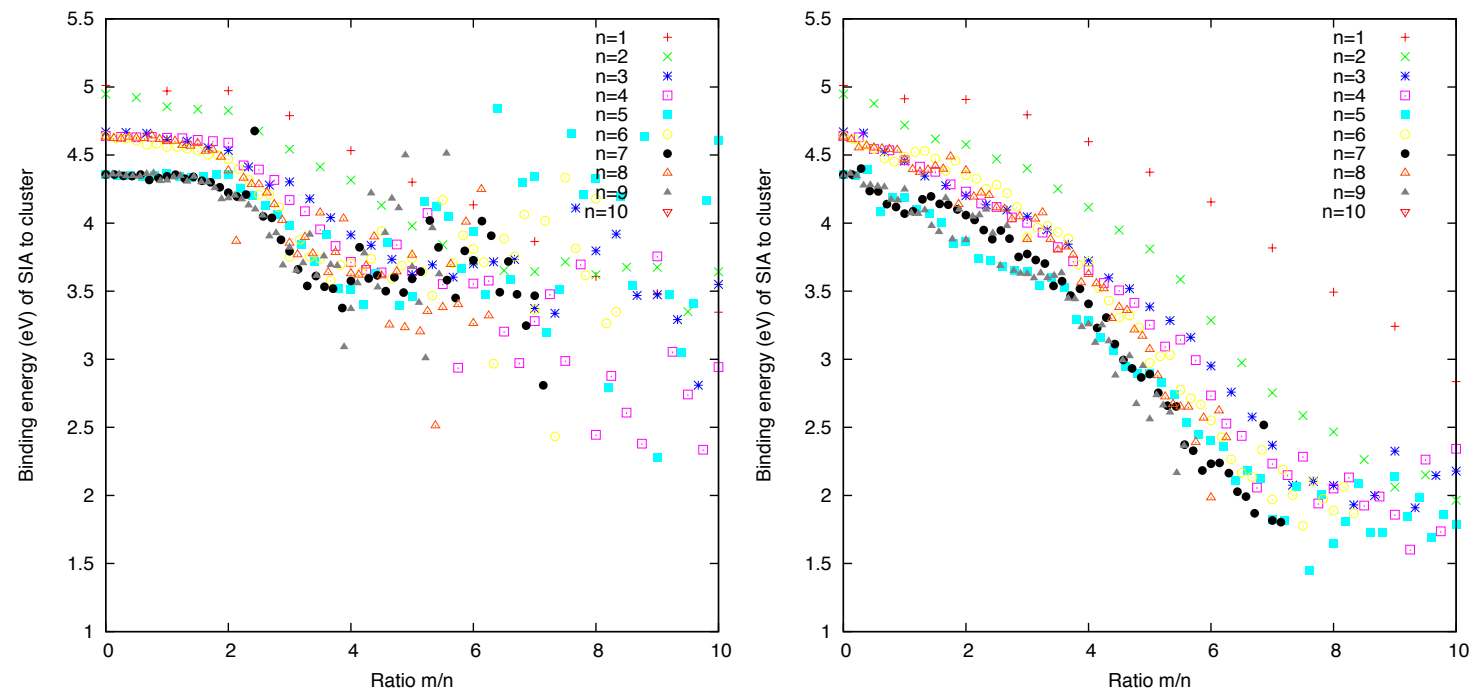

Figure 25: Binding energy of a neighboring iron atom to the cluster for potentials B (left) and $\mathrm{B}^{\prime}$ (right). Ratio $m / n$ is shown on the abscissa, binding energy on the ordinate, while curves indicate different values of $n$. As hydrogen inventory increases, binding of neighboring iron decreases.

where we would expect it to from the discussions of the binding energies of vacancies and SIAs - at around $m / n=4$ for potential B and $m / n=7$ for potential $\mathrm{B}^{\prime}$. Even at lower densities, hydrogen atoms are not strongly bound to the cluster and may easily be removed at working temperatures.

Ion implantation and ion-beam analysis studies on hydrogen trapping in iron $[97,8,99]$ provide a basis for comparison with our results. Vacancy traps and a large hydrogen inventory are created by bombarding bulk iron with deuterium and helium at low temperature, then the temperature is ramped up while simultaneously measuring hydrogen inventory. The experiments find trap strengths of $0.63 \mathrm{eV}$ and $0.43 \mathrm{eV}$ for 1-2 and 3-6 hydrogen atoms, respectively, about what are assumed to be monovacancies (plus an additional higher strength trap within helium bubbles). This is consistent with our findings for the binding of hydrogen.

Bubble growth may occur by absorption of vacancies or by the emission of neighboring iron atoms into the bulk. In order for the latter phenomenon of loop punching to occur, the emission of iron atoms from around the bubble must compete effectively with emission of a hydrogen atom. However, at any given ratio, hydrogen atoms are significantly less 

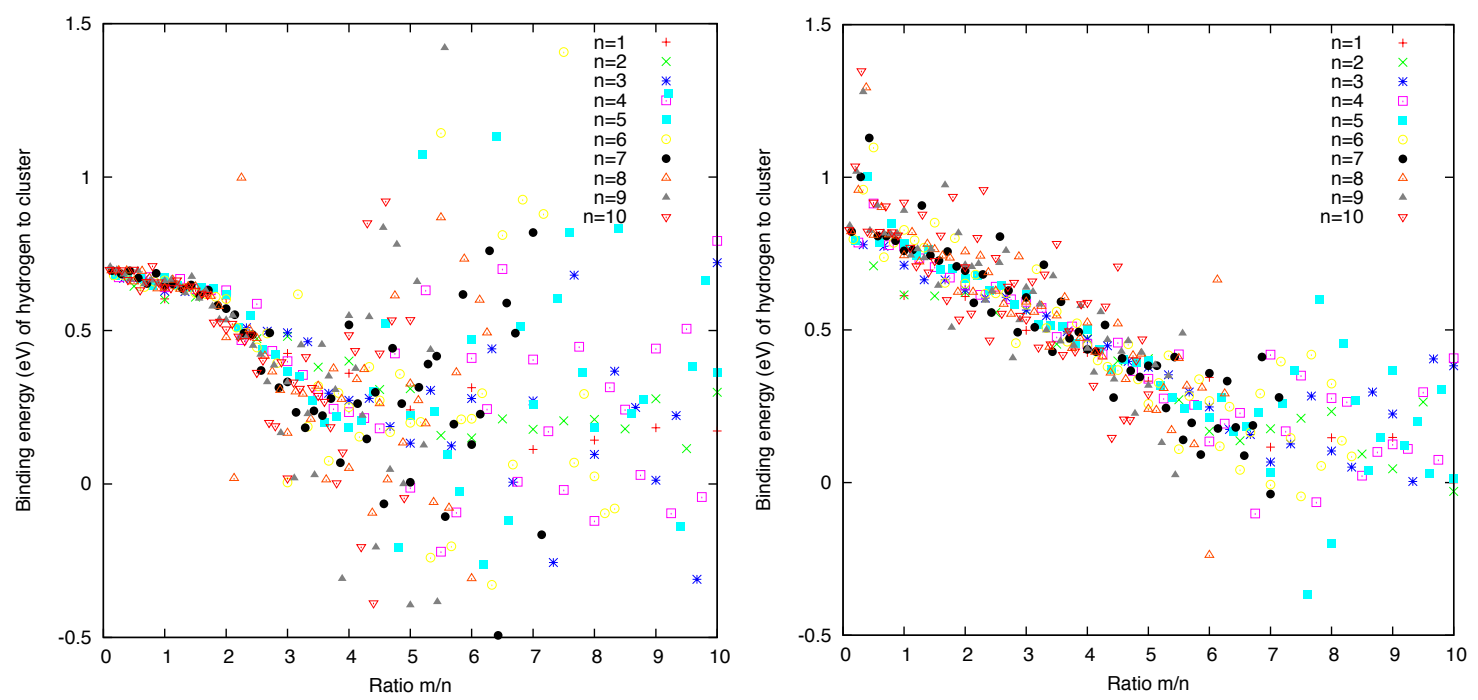

Figure 26: Binding energy of a hydrogen atom to the cluster for potentials B (left) and B' (right). Ratio $m / n$ is shown on the abscissa, binding energy on the ordinate, while curves indicate different values of $n$. As hydrogen inventory increases, the binding of hydrogen atoms decreases.

well bound to the cluster than are SIAs, so loop punching would not be expected to occur. Vacancies, which may be present in abundance in an irradiated environment, are a more likely contributor to bubble size.

\subsubsection{Multiple Hydrogen Occupancy of Monovacancies}

After analysis of the full suite of simulations above, interesting result is discovered for the case of multiple hydrogen atoms surrounding a monovacancy. The trapping of multiple hydrogen atoms at a vacancy in bcc iron is an issue that has been considered by many authors. Experiments [98, 97, 99] have shown that a single hydrogen is trapped by a vacancy in a position slightly offset by $\delta=0.4 \AA$ from an octahedral interstitial site (Osite) towards the vacant site. Six of these sites exist in the bcc structure for each vacancy, and it has been assumed that these sites will be the sites of choice when multiple hydrogen atoms are trapped by a vacancy, with sites being filled in the order shown in Figure $27^{1}$. The number of these sites that are thermodynamically favorable has been much debated, with experiment and effective-medium theory (EMT) $[63,40]$ favoring $\mathrm{H}_{6} \mathrm{~V}$ complexes, and

\footnotetext{
${ }^{1}$ The configurations pictured in Figure 27 depict what will be referred to as the $m$ Oct configurations (before relaxation), with $m$ denoting the number of hydrogen atoms in the system.
} 


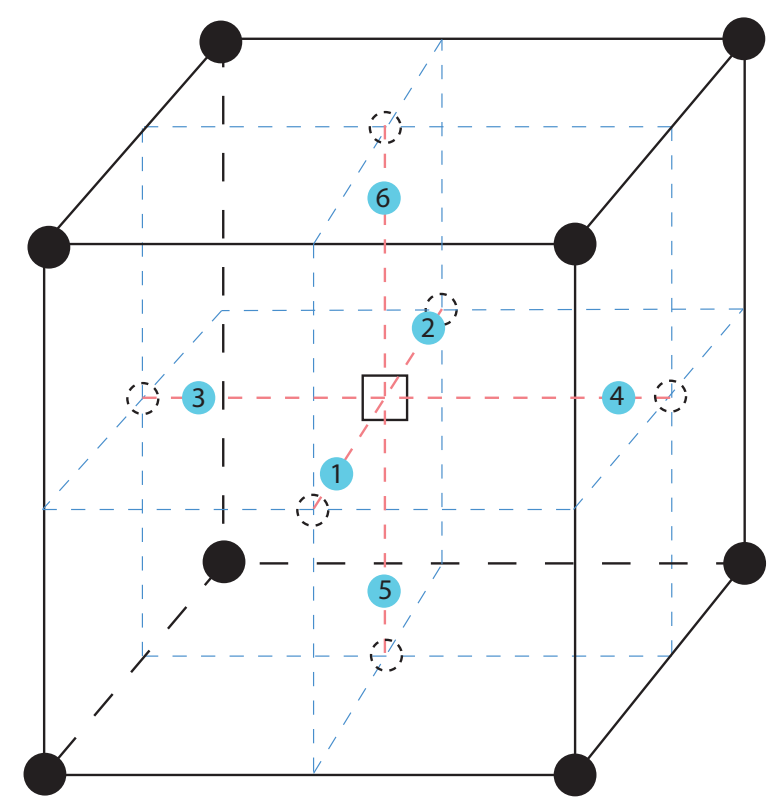

Figure 27: Generally accepted sites for six hydrogen atoms around a monovacancy (the 6 Oct configuration) in bcc iron. Iron atoms are shown in black, octahedral sites are shown with dotted circles, vacancy is shown as an empty square. The six hydrogen atoms are blue and offset from octahedral sites by $\delta$; numbers indicate the order in which atoms are added to the system to generate $m$ Oct configurations.

recent ab initio results showing $\mathrm{H}_{2} \mathrm{~V}$ complexes to be preferred [141].

However, our research indicates that there are more energetically favorable $\mathrm{H}_{m} \mathrm{~V}$ configurations than have been studied in the past, and we find new low energy structures for four, five, and six hydrogen atoms about a monovacancy. Although the method described above for locating low energy states of a cluster allows us to reach this conclusion because it makes no initial assumption about the configuration of the hydrogen atoms within a monovacancy, due to the importance of the electrons in calculations involving hydrogen, it cannot be used alone to describe energies accurately. Thus, density functional theory (DFT) calculations are subsequently performed on the low energy configurations obtained by $\mathrm{MD} / \mathrm{MC}$ methods.

The space of possible configurations for $m$ hydrogen atoms about a monovacancy $\left(\mathrm{H}_{m} \mathrm{~V}\right)$ is first searched using molecular dynamics (MD) and Monte Carlo (MC) methods, as described above. This method allows for a thorough exploration of the potential energy surface 
(PES) in a rather short time; however, due to the importance of the electrons in calculations involving hydrogen, it cannot be used alone to describe energies accurately. Thus, density functional theory (DFT) calculations are subsequently performed on the low energy configurations obtained by MD/MC methods.

The six $m$ Oct configurations are generated and minimized using the LAMMPS molecular dynamics simulator, using a $3 a_{0} \times 3 a_{0} \times 3 a_{0}$ cell with periodic boundary conditions, and Ramasubramaniam et al.'s potential B. As should be expected, we find results equivalent to those originally published by Ramasubramaniam et al. [119]. For one, two, or three hydrogen atoms, the $\mathrm{MD} / \mathrm{MC}$ method recovers the same configurations that are found when a system starting from the $m$ Oct state is minimized. The minimized position for a single hydrogen is offset from the O-site by $\delta=0.26 \AA$. When a second hydrogen is added, it occupies the offset O-site opposite from the first hydrogen, as is generally accepted. In this case, $\delta$ is lowered to $0.23 \AA$. When three hydrogen atoms are placed in the positions shown in Figure 27, two of the atoms relax off of the lines between the O-site and the vacancy while continuing to lie on a $\left(\begin{array}{lll}1 & 0 & 0\end{array}\right)$ plane, forming the configuration shown in Figure 28.

However, the MD/MC method finds new low energy configurations when considering complexes with four, five, or six hydrogen atoms. When the 4Oct configuration is created, all four hydrogen atoms lie on one plane. This is the most symmetric, and perhaps the most intuitive, configuration. After a single 0K minimization, all four hydrogen atoms will remain near plane, close to their initial positions. Two opposing atoms shift to positions slightly above the plane; the other two move slightly below it. However, when the MD/MC method is applied to the $\mathrm{H}_{4} \mathrm{~V}$ system, the hydrogen atoms are found to form a tetrahedron. This is still closely related to the offset O-sites model; if one of the in-plane hydrogen atoms is moved to either vacant offset $\mathrm{O}$-site, and the system is relaxed, our configuration is recovered. The resulting structure is seen in Figure 29.

With 5 hydrogen atoms, the two configurations are similar. However, the one found with our $\mathrm{MD} / \mathrm{MC}$ method is a slightly tilted version of the 5Oct configuration, as can be seen in Figure 30. The third, fourth, and fifth hydrogen atoms are shifted away from their offset O-sites towards tetrahedral site (T-sites). The two configurations are nearly equivalent in 

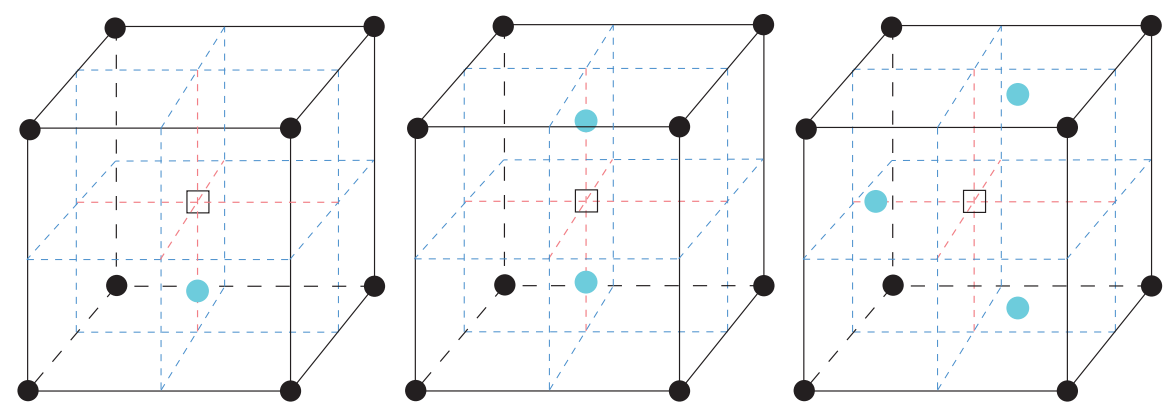

Figure 28: The positions of one, two, and three hydrogen atoms around a monovacancy, found by the MD/MC method. These are the same positions that are found when the corresponding $m$ Oct configurations are relaxed. As in Figure 27, iron atoms are shown as black circles, hydrogen atoms are blue, and the vacancy is depicted with an empty square.

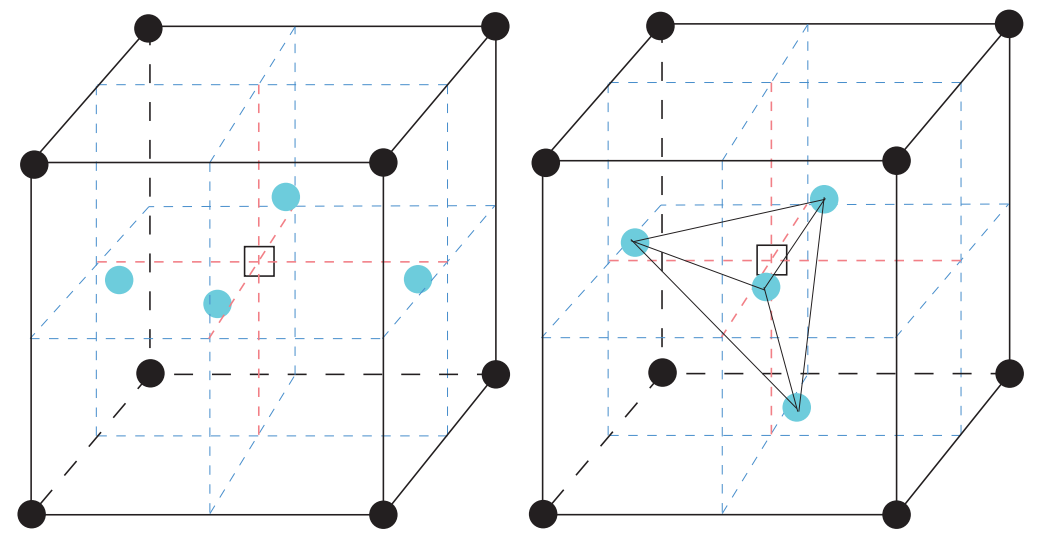

Figure 29: The positions of four hydrogen atoms around a monovacancy. 4Oct positions are shown on the left; the lower energy positions from $\mathrm{MD} / \mathrm{MC}$ are shown on the right. The conventions described in Figure 28 are followed.

energy, but ours is slightly lower.

Minimizing a 6Oct configuration will result in no structural change, other than a slightly varying $\delta$. The $\mathrm{MD} / \mathrm{MC}$ method gives a different answer. Two opposing $\mathrm{H}$ atoms are offset from octahedral positions by only $\delta=0.052 \AA$. The other four atoms lie in a $\left(\begin{array}{lll}1 & 0 & 0\end{array}\right)$ plane, slightly offset from tetrahedral positions by $\beta \sim 0.12 \AA$ and equidistant from each other. This is shown in Figure 31, the 2Oct4Tet configuration.

It is important to remember that our minimization and positioning algorithms make no initial guesses about the ideal positions for the hydrogen atoms; instead, hydrogen atoms are randomly inserted into the space around the vacancies and allowed to find the lowest energy positions through Monte Carlo methods. To quantify these results, we compared 

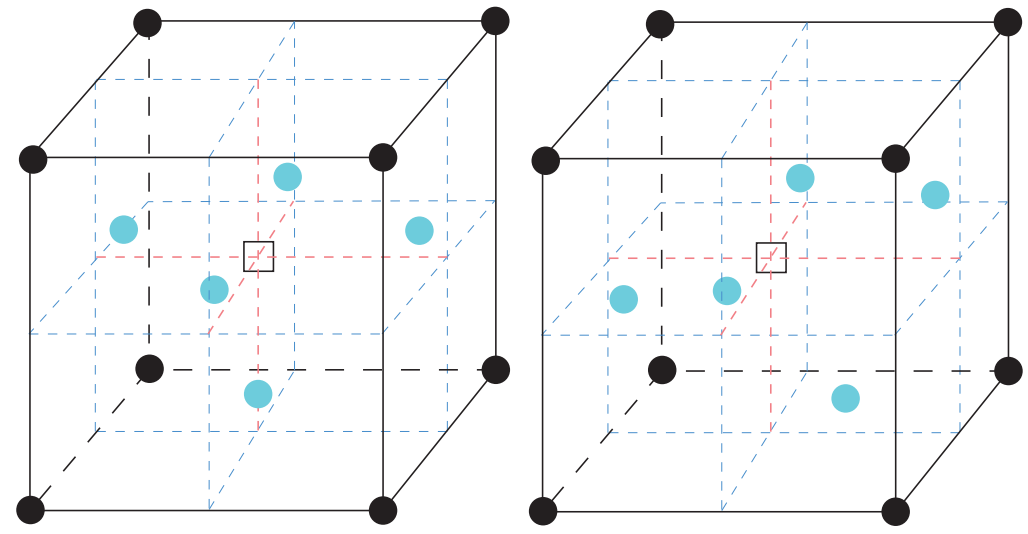

Figure 30: The positions of five hydrogen atoms around a monovacancy. 5Oct positions are shown on the left; the lower energy positions from $\mathrm{MD} / \mathrm{MC}$ are shown on the right. The conventions described in Figure 28 are followed.
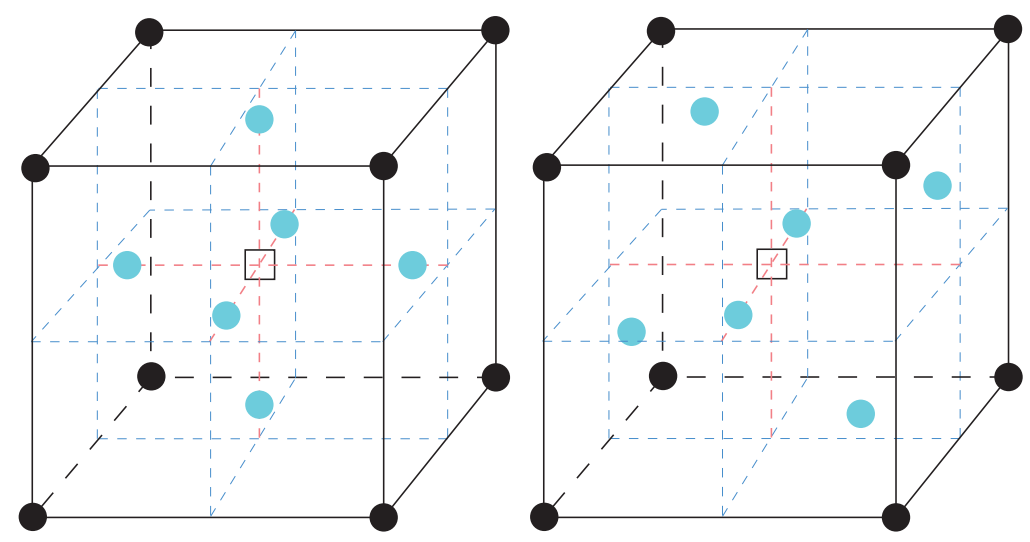

Figure 31: The positions of six hydrogen atoms around a monovacancy. 6Oct positions are shown on the left; the lower energy 2Oct4Tet configuration is shown on the right. The conventions described in Figure 28 are followed. 
Table 3: Energies (eV) found using molecular dynamics. Note that total energies $E_{T}$ and formation energies $E_{f}$ (Equation 19) are lower when $m \geq 4$ for the MD/MC states than for the corresponding $m$ Oct configurations.

\begin{tabular}{|c||c|c|c||c|c|c|}
\hline \multicolumn{1}{|c||}{} & \multicolumn{3}{c||}{$m$ Oct Positions } & \multicolumn{3}{c|}{ MD/MC Positions } \\
\hline$m$ & $E_{T}$ & $E_{f}$ & $E_{B}(\mathrm{H})$ & $E_{T}$ & $E_{f}$ & $E_{B}(H)$ \\
\hline 1 & -213.641 & 1.417 & 0.611 & -213.641 & 1.417 & 0.611 \\
2 & -216.323 & 1.105 & 0.611 & -216.323 & 1.105 & 0.611 \\
3 & -218.827 & 0.971 & 0.432 & -218.827 & 0.971 & 0.432 \\
4 & -221.197 & 0.971 & 0.299 & -221.255 & 0.913 & 0.357 \\
5 & -223.511 & 1.023 & 0.242 & -223.543 & 0.995 & 0.217 \\
6 & -225.666 & 1.242 & 0.084 & -225.891 & 1.018 & 0.277 \\
\hline
\end{tabular}

the total system, formation, and hydrogen binding energies from our simulations and those from calculations assuming offset O-sites and experiment.

The formation energy of a $\mathrm{H}_{m} \mathrm{~V}$ complex is calculated as in Equation 19 We calculate the binding energy of the $m^{\text {th }}$ hydrogen to the $\mathrm{H}_{m-1} \mathrm{~V}$ cluster with

$$
E_{B}(\mathrm{H})=\left[E_{T}\left(\mathrm{H}_{1} \mathrm{~V}_{0}\right)-E_{T}\left(\mathrm{H}_{0} \mathrm{~V}_{0}\right)\right]-\left[E_{T}\left(\mathrm{H}_{m} \mathrm{~V}\right)-E_{T}\left(\mathrm{H}_{m-1} \mathrm{~V}\right)\right]
$$

Here, $E_{T}\left(\mathrm{H}_{1} \mathrm{~V}_{0}\right)$ is the energy of a system with a tetrahedral hydrogen interstitial, and $E_{T}\left(\mathrm{H}_{0} \mathrm{~V}_{0}\right)$ is the total system energy of a perfect block of iron. This formula is mathematically equivalent to Equation 21, however we use this form in order to directly compare with results in the literature without worrying about numerical roundoff errors. Results for $E_{T}, E_{f}$, and $E_{B}(\mathrm{H})$ for both $m$ Oct and $\mathrm{MD} / \mathrm{MC}$ configurations can be found in Table 3. Energies are equivalent for $m=1,2,3$ since the configurations are identical. Total system energies are lower for our configurations than for $m$ Oct configurations when $m \geq 4$; formation energies are also lower.

To confirm our results, we performed $a b$ initio calculations as well. We took both the configurations found by $\mathrm{MD} / \mathrm{MC}$ and the $m$ Oct configurations and analyzed their energetics with DFT. We find that our calculations on the $m$ Oct states are consistent with what has previously been published [141]. Again, we find that our MD/MC configurations have slightly lower system energies than the $m$ Oct configurations in the literature. We compare our results to those available in the literature; namely, Tateyama and Ohno's DFT calculations on $m$ Oct configurations [141]. Hydrogen binding energy results can be seen in Table 
Table 4: Hydrogen binding energies $E_{B}(\mathrm{H})$ in $\mathrm{eV}$ from previously published DFT calculations, the DFT calculations done for this work, EMT, and experiment.

\begin{tabular}{|c||c|c|c|c|}
\hline$m$ & DFT - $m$ Oct [141] & DFT - MD/MC & EMT [103] & Experiment [8] \\
\hline 1 & 0.559 & 0.584 & 0.83 & 0.63 \\
2 & 0.612 & 0.607 & 0.79 & 0.63 \\
3 & 0.399 & 0.384 & 0.54 & 0.43 \\
4 & 0.276 & 0.343 & 0.51 & 0.43 \\
5 & 0.335 & 0.297 & 0.41 & 0.43 \\
6 & -0.019 & 0.002 & 0.42 & 0.43 \\
\hline
\end{tabular}

4.

The $m^{\text {th }}$ hydrogen can be considered to be exothermically bound to a monovacancy if its binding energy is greater than the heat of solution of $\mathrm{H}_{2}$ in iron. The heat of solution of hydrogen in iron from experiment is $0.29 \mathrm{eV} /$ atom [81]. DFT calculations find the heat of solution to be $0.32 \mathrm{eV} /$ atom. Using this value, Tateyama shows that up to three hydrogen atoms are exothermically bound, while the fourth has a binding energy less than the heat of solution. An addition of the fifth hydrogen atom again results in a slight bonding.

In contrast, we show that each additional hydrogen is less well bound than the one before it, instead of predicting a jump in binding energy when adding a hydrogen atom to the $\mathrm{H}_{4} \mathrm{~V}$ cluster. Binding energies are exothermic for up to four hydrogen sites, when arranged in a tetrahedron shape instead of a plane. For both the 6Oct configuration and 2Oct4Tet, the sixth atom has a binding energy close to zero.

Earlier experimental results from ion beam implantation and hydrogen detrapping studies, demonstrated that there are two release stages of hydrogen from single vacancies, at 0.63 and $0.43 \mathrm{eV}[97,8]$. The former is postulated by these practitioners to be related to 1 or $2 \mathrm{H}$ atoms being trapped at a monovacancy, while the latter corresponds to the presence of 3-6 $\mathrm{H}$ atoms. Effective medium theory (EMT) was proposed to explain these experimental results, which suggests that all six sites are exothermic for hydrogen occupancy [104, 103].

The binding energies from EMT, however, are significantly higher than the experimental results. Our values, on the other hand, agree rather well with experiment. For one or two hydrogen atoms, we find hydrogen binding energies of 0.584 and $0.607 \mathrm{eV}$, which match quite well to the second stage of release seen in experiment at $0.63 \mathrm{eV}$. Our values of 0.384 


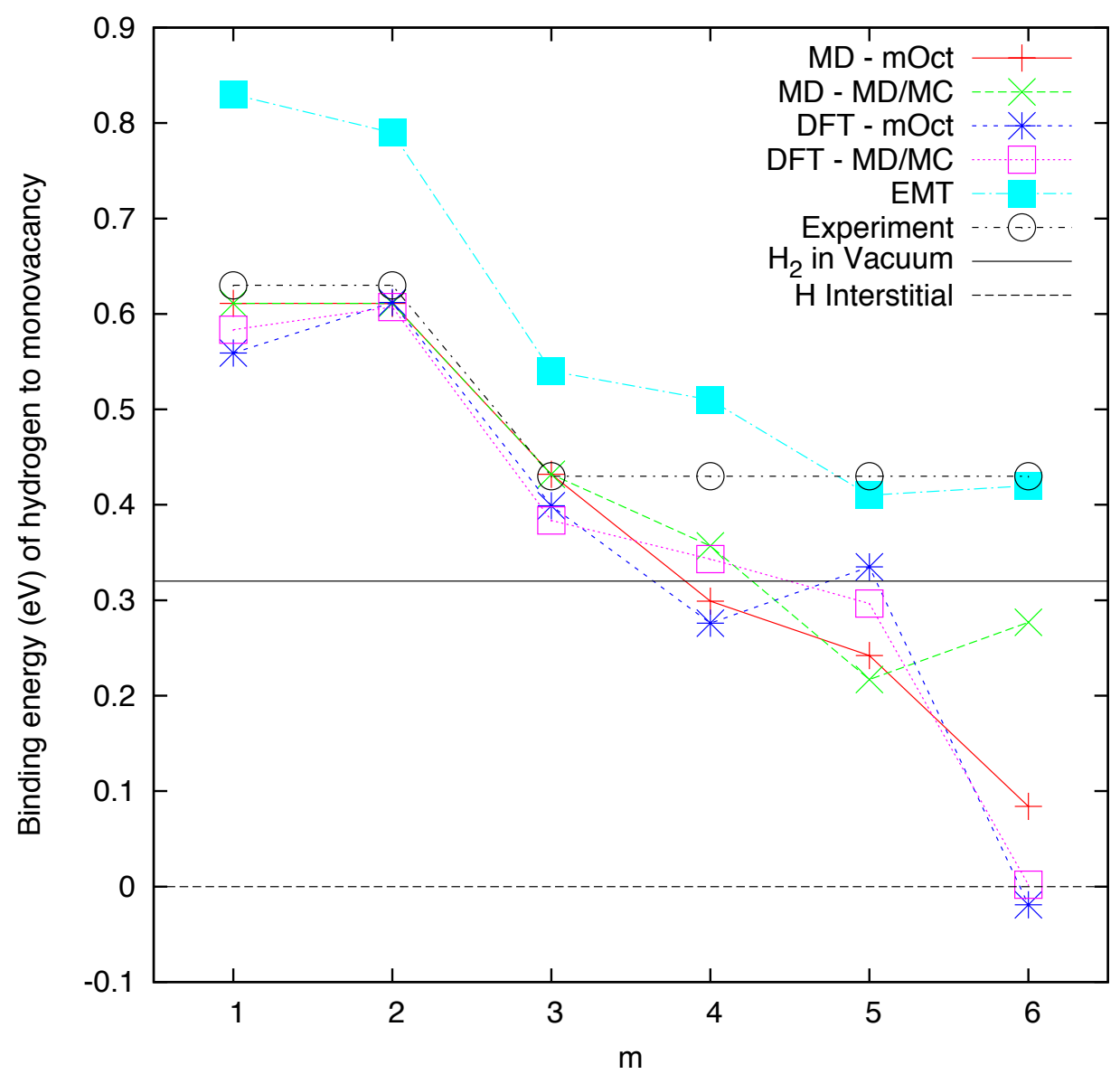

Figure 32: The binding energy of the $m^{\text {th }}$ hydrogen atom to a $\mathrm{H}_{m-1} \mathrm{~V}$ cluster. The data points marked with "MD" show data for $E_{B}(\mathrm{H})$ from Table 3, while those marked "DFT", "EMT", and "Experiment" show the data from Table 4. The energies of a hydrogen atom at an interstitial site and in an $\mathrm{H}_{2}$ molecule in vacuum (the heat of solution, as calculated by DFT) are pictured as horizontal lines.

and $0.343 \mathrm{eV}$ for the binding of the third and fourth hydrogen atoms are close to the experimentally observed release stage at $0.43 \mathrm{eV}$. Unlike the EMT results, we do not see binding of the fifth and sixth hydrogen atoms.

\subsection{Discussion}

The question of whether or not hydrogen gas in bubbles can be adequately modelled with an embedded-atom method potential is a valid one. Hydrogen, as the lightest of elements, has important quantum mechanical properties that are not directly simulated in this study. There will surely be some nuances of interaction that cannot be simulated perfectly without accounting for individual electrons. However, the size limitations of $a b$ initio methods make 
studying this problem prohibitively computationally expensive at this point in time. The present authors believe that the interatomic potentials we are using adequately reproduce the important interactions that will take place within a hydrogen bubble. The nature of $\mathrm{H}-\mathrm{H}$ interactions within metals have been studied by various authors. Effective medium theory [105] predicts that hydrogen within a monovacancy will not occupy the center, because of the below optimum value of electron density. Hybridization with the iron occurs, leaving room for multiple hydrogen atoms to occupy the vacancy; however, they will repel each other. This was recently confirmed in ab initio calculations $[141,140]$ which show Fe $3 d$ $\mathrm{H} 1 s$ hybridization, in which electron transfer occurs from an iron atom to a hydrogen. Similarly, an $\mathrm{H}_{2}$ molecule inserted into a vacancy will dissociate, suggesting that hydrogen molecules will not survive in small voids; this effect grows stronger with additional hydrogen occupancy. The interatomic potentials we have used in this study replicate this behavior, having been fit to additional density functional theory calculations. If hydrogen sometimes does exist in a molecular state inside a void, it will dissociate when approaching the iron on the surface. Thus, we can assume that we will capture the most important energetics of a cluster as long as the interactions between hydrogen and iron are properly described.

Although no studies on the specific energetics of hydrogen-vacancy clusters in bcc iron exist in the literature, there are some on helium bubbles $[92,91,77,53]$. These studies show good agreement with experimental results, which gives us additional confidence that our similar system can be modelled well with these methods. The helium bubbles in iron show the same general trends in binding energy as the hydrogen bubbles we have modelled, with helium tending to stabilize voids. However, the binding of helium to clusters is significantly stronger than the binding of hydrogen; it is comparable to the binding of SIAs. Morishita et al. [91] find that bubbles can support ratios of up to 6 helium atoms per vacancy, at which point SIA-vacancy pairs are created on the edges of bubbles.

Clusters simulated with potential $\mathrm{B}^{\prime}$ are much more easily minimized to the lowest energy state than those created with potential B. This is due to the more complex nature of the PES generated by potential B. Although the time per iteration is shorter with potential B than potential $\mathrm{B}^{\prime}$, the increased value of $q$ that is needed cancels this benefit. However, 
the interactions described by potential B seem to be more physically relevant than those of $\mathrm{B}^{\prime}$, based on the formation energy curves seen in Figure 23. In the original paper describing these potentials $[119,121]$, only data and fitting for potential $\mathrm{B}$ is given, so potential $\mathrm{B}^{\prime}$ needs to be characterized. Our calculations provide data on both potential B and B's performance with strongly interacting $\mathrm{H}$ clusters which is lacking in the original parameterization.

Binding energy trends are generally consistent between both potential variants. Vacancies become more tightly bound to clusters as the ratio $m / n$ is increased, while both SIAs and hydrogen atoms become more loosely bound. Results from potential B indicate that clusters can support a maximum ratio of $\sim 4$; potential $\mathrm{B}^{\prime}$ allows for ratios up to $\sim 7$. However, the formation energy curves generated by the two potentials differ, with potential B providing a trend that more closely matches the observed physics. Thus, the former should be taken as a limiting factor for hydrogen ratio in bcc iron.

Based on these basic energetics, bubble growth by vacancy absorption would be a more likely mechanism than loop punching or similar modes. Hydrogen seems to be loosely bound to clusters even at low densities, and the question remains whether it will be bound long enough for stabilizing effects to be realized at temperatures seen in reactor structural materials. However, hydrogen's effect on the binding of self-interstitials may be relevant to the HELP theory of hydrogen embrittlement as well. If interstitials are less well-bound in the presence of hydrogen, dislocation mobility may be increased. Our results for hydrogen binding agree well with experimental ion-beam studies of hydrogen trapping energies, as discussed in the previous section. However, our simulations allow for more precision in calculating energies and allow us to exactly determine the void sizes and bubble ratios involved in creating the traps.

Finally, we have discovered new low energy configurations for four, five, and six hydrogen atoms surrounding a monovacancy in bcc iron and confirmed these results with ab initio calculations. All previous calculations and theories assumed that up to 6 hydrogen atoms surrounding a monovacancy would reside in offset O-sites. However, we made no initial assumptions about the positions of the hydrogen atoms, allowing for an unbiased search for the lowest energy configurations. 
The question of how many hydrogen atoms can exothermically exist about a monovacancy has been addressed by calculating binding energies of the $m^{\text {th }}$ hydrogen to a $\mathrm{H}_{m} \mathrm{~V}$ cluster. Our simulations agree with previous results [141] that show that the $\mathrm{H}_{6} \mathrm{~V}$ complex is not the dominant complex in bcc iron, in contrast with earlier experimental and effective medium theory results. However, unlike those previous results, our DFT results show that up to four hydrogen atoms may be exothermically bound to a monovacancy. As more hydrogen atoms are added to a vacancy, each is less well bound than the ones before. This trend is different than when mOct configurations are assumed; in this case, the fourth hydrogen atom is not bound, while the fifth is slightly bound when referenced to the heat of solution. Our low energy configurations provide new insight into the role of vacancy-hydrogen complexes in materials degradation.

The energetics of $\mathrm{H}_{m} \mathrm{~V}_{n}$ clusters in bcc iron have been investigated using a combination of molecular dynamics and Monte Carlo techniques, with two different interatomic potentials. The use of empirical potentials allows for larger scale simulations than are currently possible with ab initio methods. However, the simulations can still model individual atoms, which gives much more detail than experiment can provide. This detail is essential for the fundamental understanding of how interactions between defects can affect macroscopic properties. Significant differences in the potentials can be seen, but overall trends in binding energies are consistent between both. Hydrogen is seen to have a stabilizing effects on voids, which supports the idea that increased vacancy concentration and clustering may be responsible for hydrogen embrittlement. Although hydrogen is relatively weakly bound to voids, its presence may play a significant role in determining macroscopic properties. 


\section{CHAPTER IV}

\section{HYDROGEN - HELIUM BUBBLES}

\subsection{Motivation and Literature Review}

Hydrogen and helium are known to have detrimental effects on structural materials, which can be aided by irradiation. This is expected to be a significant problem for future generations of nuclear reactors. This is especially true of fusion reactors $[12,123]$, in which isotopes of hydrogen provide fuel for the fusion reaction, and helium is created as a transmutation byproduct. There are many theories on what mechanisms are responsible for the deleterious effects of $\mathrm{H}$ and $\mathrm{He}$ on structural materials $[10,63,64,18,24,35,73,101,107,116]$, ranging from concentration at crack tips to movement along grain boundaries. Whatever the underlying cause, it is well known that either element can contribute to embrittlement and swelling of irradiated materials, with undesirable consequences [102].

In an irradiated system, an above-average concentration of vacancies may assist these processes. Hydrogen and helium diffusing through the material can become trapped at small clusters of vacancies, providing internal stabilization [72]. This results in a decreased likelihood of recombination of self-interstitials with these vacancies, and increased plasticity of the material as a whole. There is a long history of computational study of both hydrogen and helium [145], and in particular, there have been several atomistic scale studies of the energetics of bubbles containing either pure hydrogen $[62,55,141,140]$ or pure helium $[92,38,77,53,133,43]$ in $\alpha$-iron. These studies confirm the idea that the gas stabilizes vacancy clusters, as can be seen in Figures 24 and 25. Vacancies are more tightly bound to the clusters as the inventory of gas increases, while the iron atoms that neighbor the cluster are less tightly bound to their lattice sites and are more likely to be emitted into the bulk as self-interstitials.

While either hydrogen or helium alone may be detrimental, there is evidence to suggest that when both elements are present, synergistic effects occur. It has been shown that 
when steel alloys are irradiated with both hydrogen and helium ion beams simultaneously (along with an iron ion beam to induce damage), the swelling of the material and the size of cavities increased significantly compared to samples irradiated with only one of the two elements $[152,138]$. Synergistic effects have also been observed in similar studies on other bcc transition metals, such as vanadium [124]. In all these cases, an increased dislocation loop density was also observed under simultaneous irradiation.

Additionally, although the solubility of hydrogen in iron is expected to be quite low by Sievert's law, it has been shown that when helium is also present within the crystal, hydrogen trapping is significantly enhanced even for periods of years after irradiation [142, 45, 44]. It has been theorized that this is due to increased trapping of hydrogen at helium bubbles. This trapping has been observed in metals [7, 97], and attributed to the hydrogen being attracted to the stress field of the pressurized helium bubble [1]. However, there have been no atomistic studies focusing on bubbles containing both hydrogen and helium in iron.

In this work, we present an atomistic study of vacancy clusters containing both pure hydrogen and hydrogen plus helium in bcc iron. We first introduce an interatomic potential suitable for describing the interactions between hydrogen and helium. This potential is used to perform a detailed analysis of the configurations and energetics of a variety of bubbles. We find that the synergistic effects on bubble properties can be explained not through a direct interaction between hydrogen and helium, but through the phenomenon of loop punching. We show that the presence of hydrogen makes loop punching a more energetically favorable event for a bubble with the required amount of helium. In turn, the growth of the bubble results in a larger free surface onto which hydrogen may be bound.

\subsection{Methods}

We simulate clusters of $m$ hydrogen, $j$ helium, and $n$ vacancies $\left(\mathrm{H}_{m} \mathrm{He}_{j} \mathrm{~V}_{n}\right)$ in bcc iron. In order to extract the energetic properties of these clusters, it is desirable to find the lowest energy configuration of the gas atoms with the voids. We achieve this through iterations of conjugate gradient relaxation and Monte Carlo criteria, using the methods described in Section 3.2 and simply also including $j$ helium atoms in the initial random distribution of 
gas atoms.

A suite of interatomic potentials is required to describe all of the interactions between hydrogen, helium, and iron. For the Fe-Fe and Fe-H interactions, we use potential B from Ramasubramaniam et al. [119, 121], which takes the Fe-Fe interactions from the potential of Ackland et al. [3, 83]. For the Fe-He interations, we use the repulsive pair potential of Juslin and Nordlund [69], and for the He-He interactions, Beck's potential is used [6]. For the H-He interactions, we use our own interatomic potential, described in Section 1.2.1.5.

\subsection{Results}

The idea that bubbles may build up sufficient internal pressure to cause neighboring matrix atoms to be ejected from their lattice sites was proposed many years ago [49], and described in further detail later [35, 146]. This athermal process, known as loop punching, allows for bubble growth even when vacancy absorption is unlikely, perhaps at low temperatures when vacancies are nearly immobile. When neighboring atoms are ejected from their lattice site, Frenkel pairs are created; the vacancy becomes a part of the cluster, while the interstitial may move through the lattice. It is known that helium can build up to high enough pressures within a bubble to cause this occurrence; hydrogen on the other hand, will become unbound from a bubble long before it can induce loop punching. Additionally, experimental studies show an increase in interstitial loops and bubble-loop complexes after irradiation with helium [21].

The process by which bubbles are created progresses in two stages: nucleation and growth. Helium interstitials may cluster together, acting as self-traps [156], or they may become trapped at vacancies. Studies on bubble nucleation show that an initial vacancy is not required for nucleation [90]; instead, the binding between several helium atoms is strong enough to punch out the first interstitial. Additional helium atoms then become trapped at the embryonic bubble, eventually resulting in increased pressure and growth [29].

Although the name "loop punching" is suggestive of the thought that an entire interstitial dislocation loop with a radius equal to that of the bubble would be emitted at once 


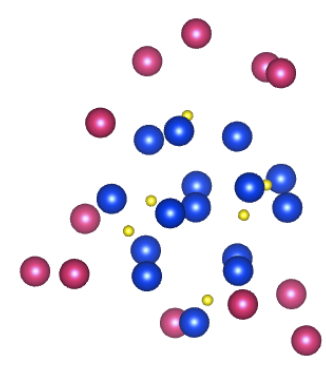

(a) $\mathrm{H}_{12} \mathrm{He}_{15} \mathrm{~V}_{6}$ cluster

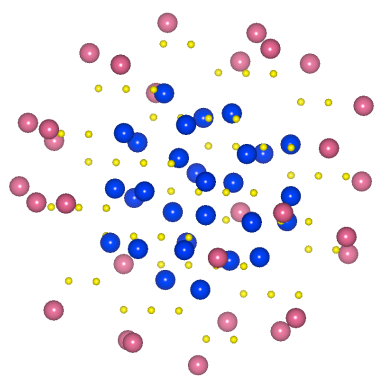

(b) $\mathrm{H}_{30} \mathrm{He}_{30} \mathrm{~V}_{59}$ cluster

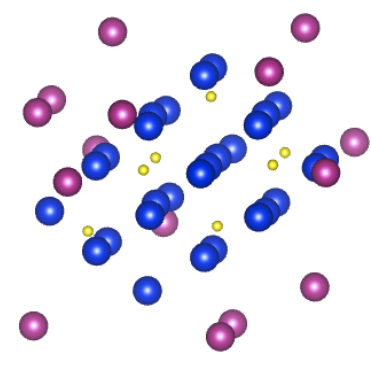

(c) $\mathrm{H}_{15} \mathrm{He}_{28} \mathrm{~V}_{7}$ cluster

Figure 33: The low energy configurations of hydrogen-helium-vacancy clusters are shown. Vacant lattice sites are pictured as small yellow circles, hydrogen atoms are pink, and helium atoms are blue. Surrounding iron atoms are not pictured. The helium atoms form the core of the bubble, with the hydrogen atoms making up the shell. In general, the helium was not well-ordered, but occasionally highly ordered states such as in 33(c) were seen.

$[35,17]$, some recent simulations show that the loop may be created through a series of emissions of single interstitials over time [42]. These individual interstitials are initially bound to the cluster. Whether full loops or single interstitials are emitted may be a function of the bubble size.

\subsubsection{Bubble Structure}

In all the simulations performed, the lowest energy configuration of the gas atoms within the bubble took approximately the same form, regardless of the bubble size. The core of the bubble was comprised of helium, surrounded by a shell of hydrogen atoms.

This structure does not seem to result from any particular interaction between hydrogen and helium. If either element is removed, the other maintains essentially the same structure. That is, in a bubble containing only hydrogen, the hydrogen will attach to the free surface, leaving the core empty. Once the surface of the bubble is effectively covered, the remaining hydrogen is emitted into the bulk. In a pure helium bubble, the gas maintains a distance from the iron atoms comprising the surface of the bubble, as found in the simulations of Stewart et al. [133]. Examples of these structures are shown in Figure 33. Additionally, although the helium in a cluster was not generally well ordered, occasionally highly-ordered states were observed as in Figure 33(c).

For the majority of this work, we consider bubbles made up of six vacancies. Since 


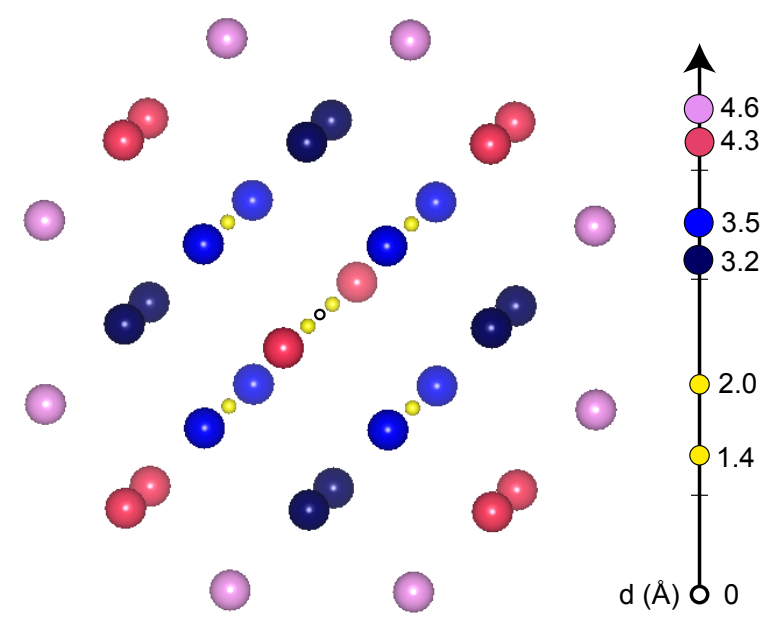

Figure 34: The structure of the unrelaxed configuration of a cluster of 6 vacancies. The vacant lattice sites are shown in yellow, and the first four shells of neighboring iron atoms are also shown, sorted by color. The geometric center of the bubble is shown as a small white circle, and the distance $d$ (Angstroms) at which members of each group reside from the center is shown on the right. We use the dark blue atoms, which reside at a distance or $\sim 3.2 \AA$ from the geometric center, to define the inner and outer radii of the cluster as described in the text.

our bubble are rather small and may not be considered fully spherical, defining a radius is not straightforward. However, the iron atoms that surround the six vacancies form shells, as can be seen in Figure 34. Before gas atoms are added to the bubble, eight iron atoms reside closest to, and at the same distance from, the geometric center of the bubble in a symmetric configuration. As we add gas atoms to the bubble, the symmetry is broken. Thus, we refer to the inner and outer radii as the distances between the geometrical center of the bubble and the iron atoms of this first shell that are closest to and farthest from that point, respectively.

Figures 35 and 36 show profiles of where different elements are located within the clusters. Both the inner and outer radii are shown, as well as a profile of where the hydrogen is located. As can be seen from the red curves in Figure 35, hydrogen has little ability to enlarge the radius of a bubble. As hydrogen inventory is increased, only a very slight increase in the radius is observed. At $m=20$, a sharp decrease in inner radius is seen, while the outer radius stays roughly constant. The hydrogen profile explains this behavior. When $m<19$, all of the hydrogen well is within the inner radius. At $m>19$, at least one 


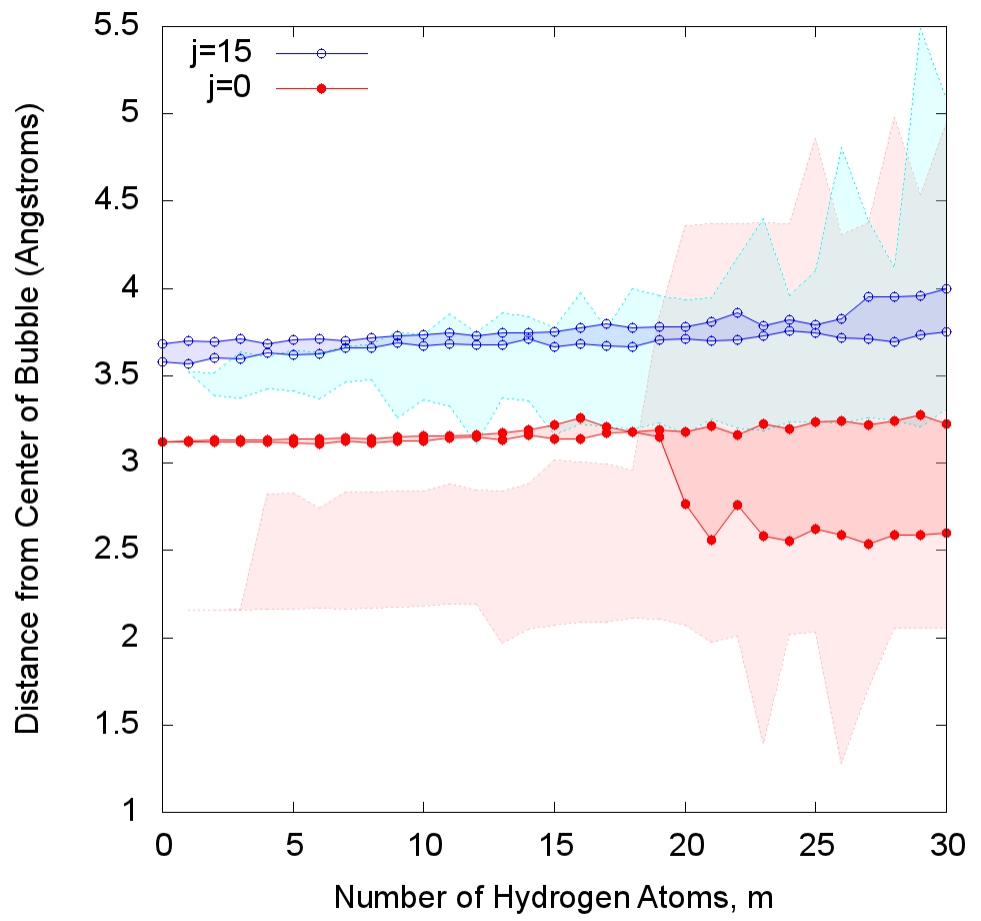

Figure 35: The radii in Angstroms of a $\mathrm{H}_{m} \mathrm{He}_{j} \mathrm{~V}_{6}$ cluster as $m$ is increased; $j$ is constant with a value of 0 (red, filled points) or 15 (blue/cyan, hollow points). The two lines for each curve show inner and outer radii, as described in the text. Lightly colored shaded areas show the geometric profile of the hydrogen atoms within the clusters and follow the curves that are similarly colored. Helium causes an increase in radius of the bubble, while hydrogen alone does not. The addition of helium to the bubble changes the hydrogen's positioning, but allows more hydrogen to remain close to the interior.

hydrogen atom is residing outside the outer radius. This hydrogen atom sits behind an iron atom and pushes it towards the center of the bubble. In contrast (as seen in the blue curves of Figure 35), when 15 helium atoms are also located within the cluster, internal pressure causes an overall increase in radius. The bubble is fairly symmetric with inner and outer radii having nearly the same value, and the radius does not increase further as hydrogen is added. However, the hydrogen profile is affected - hydrogen tends to reside closer to the iron atoms than when helium is absent, but remains largely within the inner radius. More hydrogen remains close to the bubble surface when helium is present than when it is absent. As will be discussed below, this is because the hydrogen has a greater binding energy with helium present than without it, when $j \geq 19$. 


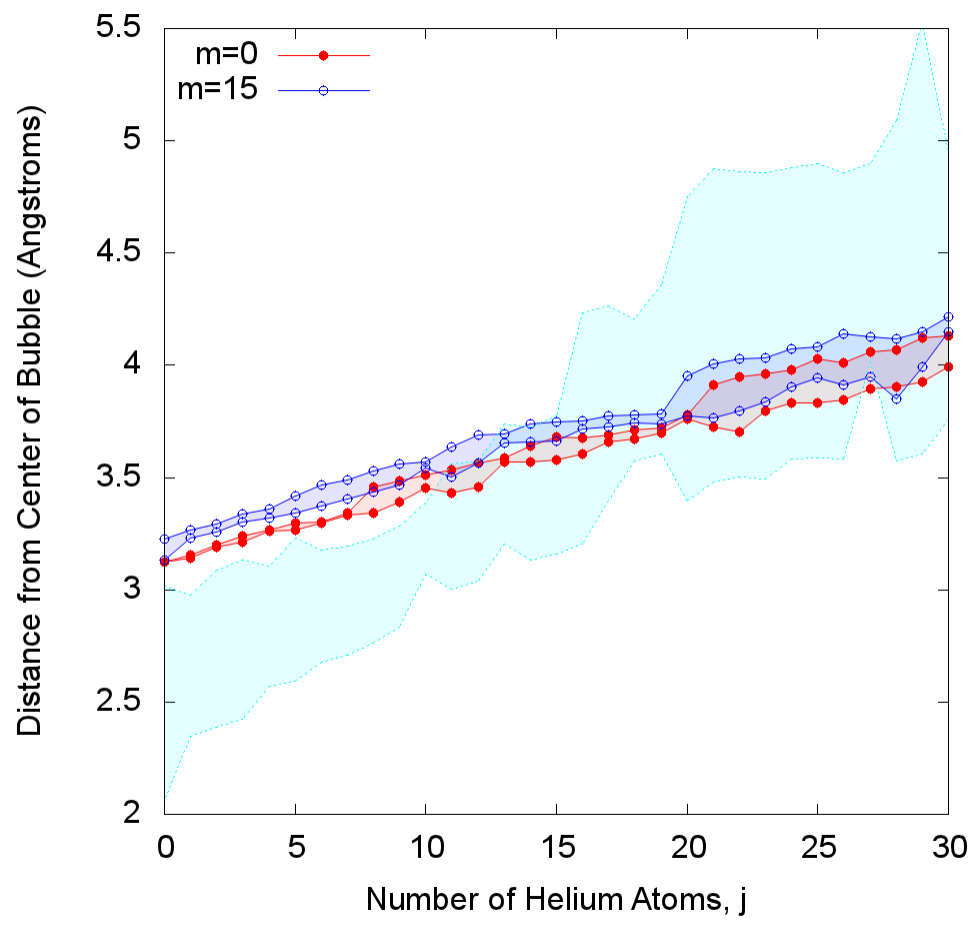

Figure 36: The radii in Angstroms of a $\mathrm{H}_{m} \mathrm{He}_{j} \mathrm{~V}_{6}$ cluster as $j$ is increased; $m$ is constant with a value of 0 (red, filled points) or 15 (blue, hollow points). The two lines for each curve show inner and outer radii, as described in the text. The light cyan area shows the geometric profile of hydrogen atoms within the latter cluster. The radius of the bubble is an increasing function of the helium inventory. As the helium inventory is increased, some of the hydrogen is pushed outside the first neighbor shell of iron. 
Figure 36 shows similar profiles, but for bubbles with increasing $j$ and constant $m$. Unlike for hydrogen, increasing helium results in an increasing radius. Having 15 hydrogen atoms in the bubble seems to further increase the radius, but only very slightly. As helium is increased, the hydrogen in the cluster moves from being primarily inside the inner radius to primarily outside the outer radius. We see that when the cluster gains 21 helium atoms, it becomes slightly less symmetric; additionally, the hydrogen moves a bit farther into the bulk, but it still attracted to the cluster.

\subsubsection{Interaction between $\mathrm{H}$ and $\mathrm{He}$}

The direct interactions between hydrogen and helium can be characterized through their binding energies to $\mathrm{H}_{m} \mathrm{He}_{j} \mathrm{~V}_{6}$ clusters, as seen in Figure 37. For these simulations clusters with $0 \leq m \leq 30$ and $0 \leq j \leq 30$ were considered. It should be noted that the Monte Carlo process described in Section 3.2 is essential to obtaining the following results. Since binding energies are on the order of $1 \mathrm{eV}$, small deviations from the global minima can result in large errors.

The formation energy for a particular cluster can be calculated by taking the difference between a cluster's energy and the sum of the energies of the constituent parts:

$$
E_{f}\left(\mathrm{H}_{m} \mathrm{He}_{j} \mathrm{~V}_{n}\right)=E_{t o t}\left(\mathrm{H}_{m} \mathrm{He}_{j} \mathrm{~V}_{n}\right)-\left\{(N-n) E_{c o h}^{F e}+m E_{c o h}^{H}+j E_{c o h}^{H e}\right\}
$$

Here, the "tot" subscript refers to total energy, while the "coh" subscript refers to cohesive energy. There are $N$ iron atoms in the system lacking defects or impurities. We use the values $E_{c o h}^{F e}=-4.013 \mathrm{eV} /$ atom, $E_{c o h}^{H}=-2.37 \mathrm{eV} /$ atom, and $E_{c o h}^{H e}=-.00714 \mathrm{eV} /$ atom. In general, adding a hydrogen atom to the cluster lowers the total system energy, while adding a helium atom raises it.

The formation energies can be used to calculate the binding of defects and atoms to the clusters. The binding energy of the $m^{\text {th }}$ hydrogen atom to a $\mathrm{H}_{m} \mathrm{He}_{j} \mathrm{~V}_{n}$ cluster is given by

$$
E_{B}(\mathrm{H})=E_{f}\left(\mathrm{H}_{m-1} \mathrm{He}_{j} \mathrm{~V}_{n}\right)+E_{f}^{H}-E_{f}\left(\mathrm{H}_{m} \mathrm{He}_{j} \mathrm{~V}_{n}\right)
$$

while the binding energy of the $j^{\text {th }}$ helium atom is given by

$$
E_{B}(\mathrm{He})=E_{f}\left(\mathrm{H}_{m} \mathrm{He}_{j-1} \mathrm{~V}_{n}\right)+E_{f}^{H e}-E_{f}\left(\mathrm{H}_{m} \mathrm{He}_{j} \mathrm{~V}_{n}\right) .
$$


The formation energy of a tetrahedral hydrogen interstitial $E_{f}^{H}$ is $0.29 \mathrm{eV}$, while the formation energy of a tetrahedral helium interstitial $E_{f}^{H e}$ is much larger at $4.39 \mathrm{eV}$.

Helium appears to have a significant effect on the binding of hydrogen to clusters, as can be see in Figure 37(a). For low concentrations of hydrogen (less than about 18 atoms in the void), adding helium to the bubble decreases the hydrogen's binding energy. This is in contrast to the idea that helium directly causes hydrogen to be more tightly bound to bubbles, as has been previously suggested [97]. However, at high concentrations of hydrogen, we see the opposite effect. While hydrogen is essentially unbound from a bubble containing only hydrogen, the addition of enough helium allows the hydrogen to remain more strongly bound. This can be attributed to the fact that a large amount of helium atoms will cause an increase in radius of the bubble, resulting in a larger free surface for a given amount of hydrogen to spread out in, as was discussed in the previous section. However, we can generally say that for a bubble with a given radius and a given number of hydrogen atoms, the addition of further gas atoms of either species results in a decreased hydrogen binding to the cluster.

On the other hand, the binding of helium to the clusters is only weakly affected by the presence of hydrogen, as shown in Figure 37(b). For a given amount of helium, introducing hydrogen to the bubble barely affects the helium. Its binding is a much stronger function of the concentration of helium atoms in the bubble - as the concentration increases, helium is less well-bound.

It should be noted that the binding of helium is always much stronger than the binding of hydrogen in these simulations. While the most tightly bound hydrogen atoms are bound by less than $1 \mathrm{eV}$, the most weakly bound helium atoms are bound by more than $1.5 \mathrm{eV}$. In summary, the binding energy of hydrogen to the cluster is more strongly affected by helium's presence than vice versa. 


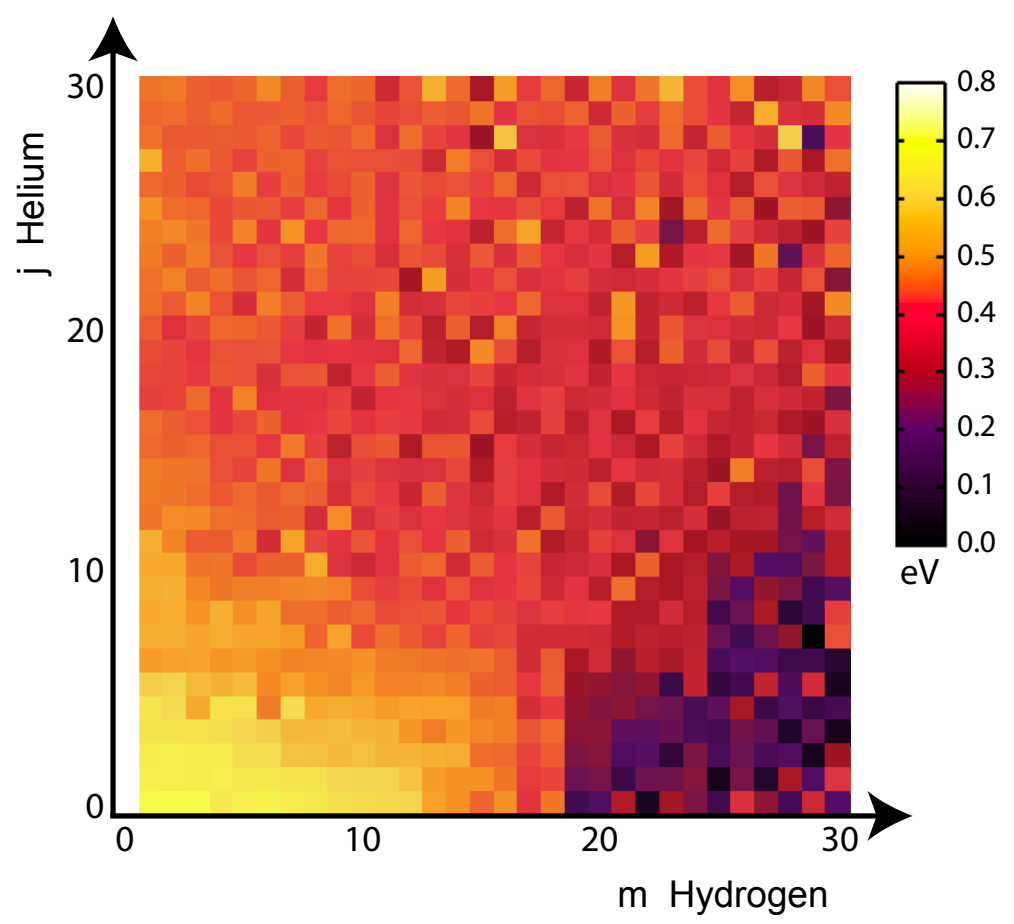

(a) Hydrogen binding energies

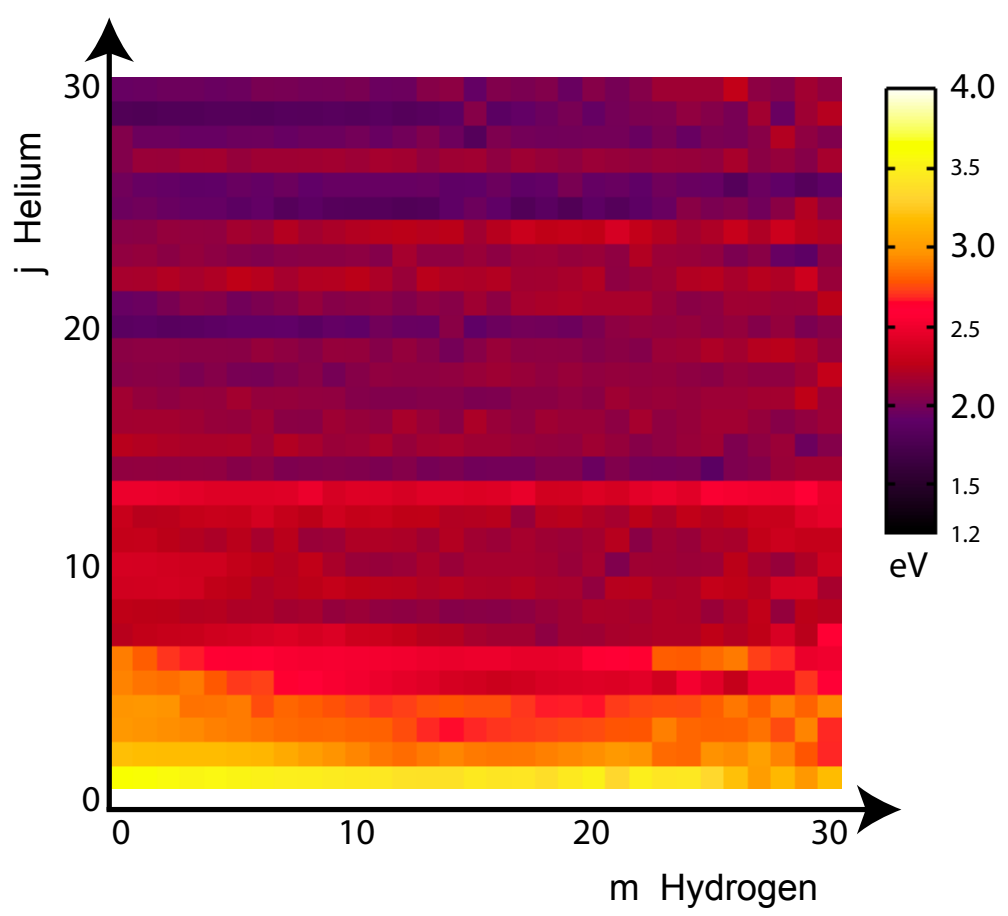

(b) Helium binding energies

Figure 37: The binding energies of hydrogen and helium to $\mathrm{H}_{m} \mathrm{He}_{j} \mathrm{~V}_{6}$ clusters. Binding energies are depicted by the respective color bars; data are in $\mathrm{eV}$. 


\subsubsection{Binding of Vacancies and Interstitials}

We can also characterize the clusters through the binding of point defects. The binding energies of vacancies and self-interstitial iron atoms are given by

$$
\begin{gathered}
E_{B}(\mathrm{~V})=E_{f}\left(\mathrm{H}_{m} \mathrm{He}_{j} \mathrm{~V}_{n-1}\right)+E_{f}^{V}-E_{f}\left(\mathrm{H}_{m} \mathrm{He}_{j} \mathrm{~V}_{n}\right) . \\
E_{B}(\mathrm{SIA})=E_{f}\left(\mathrm{H}_{m} \mathrm{He}_{j} \mathrm{~V}_{n+1}\right)+E_{f}^{S I A}-E_{f}\left(\mathrm{H}_{m} \mathrm{He}_{j} \mathrm{~V}_{n}\right),
\end{gathered}
$$

where $E_{f}^{V}=1.721 \mathrm{eV}$ and $E_{f}^{S I A}=3.529 \mathrm{eV}$.

As can been seen in Figure 38, the binding energy of a self-interstitial to a cluster of $j$ helium atoms and 6 vacancies decreases at a faster rate than does the binding of helium itself, as the helium inventory is increased. This trend has previously been observed in several studies $[92,126,77]$. Eventually, at a ratio of about three helium atoms per vacancy, selfinterstitials are less well bound than are helium atoms. Once there are several helium atoms in the cluster, vacancies are more tightly bound than either helium or self-interstitials.

Introducing hydrogen to the clusters affects these binding energies. The binding of self-interstitials is consistently lower when 15 hydrogen atoms are included in the clusters than when they are absent, across the entire range of helium inventories. The binding of helium itself is also slightly affected, primarily at low concentrations of helium. Thus the SIA binding energy curve intersects with the helium atom binding energy curve at a lower ratio than when hydrogen is not present, close to $j / n=2$. Additionally, including hydrogen increases the binding of vacancies to clusters.

\subsubsection{Loop Punching}

A necessary criterion for loop punching to occur is that the binding of the species inside the bubble to the bubble be stronger than the binding of neighboring matrix atoms to the bubble. For hydrogen in bubbles, binding is rather weak compared to the binding of selfinterstitials; hydrogen will always be emitted from the bubble before pressures can build up high enough to generate loop punching. However, for helium, loop punching is a recognized phenomenon; a cluster of helium atoms in the bulk may even punch out an initial vacancy. 


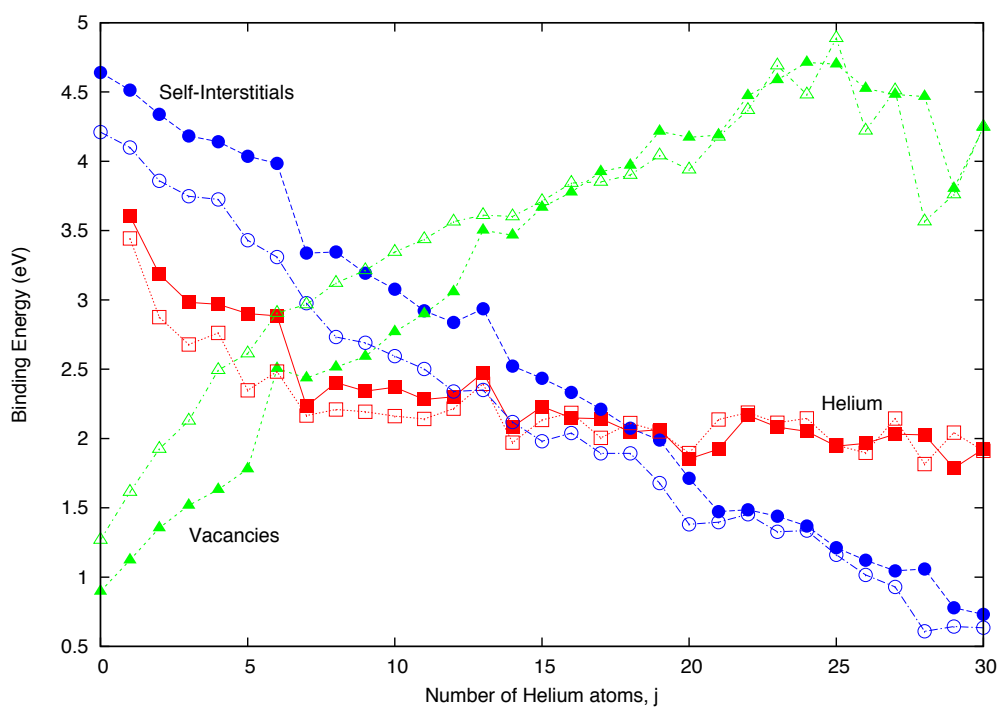

Figure 38: Binding energies (eV) of helium atoms (red squares), SIAs (blue circles), and vacancies (green triangles) to $\mathrm{H}_{m} \mathrm{He}_{j} \mathrm{~V}_{6}$ clusters. Filled data points represent pure helium bubbles $(m=0)$; hollow data points are from bubbles with $m=15$. As the helium inventory is increased, both the binding of self-interstitials and helium atoms decreases, while the binding of vacancies increases. Hydrogen exacerbates these effects, especially for low helium concentrations.

As shown in Figure 38, in this study of clusters with $n=6$, this condition occurs for the pure helium bubbles at a ratio of approximately $j / n=3$.

However, this criterion in and of itself is not sufficient to guarantee loop punching; it must also be energetically favorable for a Frenkel pair to be created. The minimum energy configurations of $\mathrm{H}_{m} \mathrm{He}_{j} \mathrm{~V}_{n}$ and $\mathrm{H}_{m} \mathrm{He}_{j} \mathrm{~V}_{n+1}$ bubbles are generated with the method discussed in Section 3.2 . To simulate loop punching, an iron atom is introduced to the latter configuration at a particular lattice site, and the new configuration is minimized. These lattice sites are chosen both near the $(n+1)^{\text {th }}$ vacancy and at large distances to determine how an interstitial created at the surface of the bubble would be bound. At each lattice site where a dumbbell was placed, the six possible orientations of a $<110>$ dumbbell are individually tested; the orientation which results in the lowest energy structure is taken as our data point. The $<110>$ family of dumbbells is the most stable in bulk bcc iron, but no restriction is made to guarantee that a $<1 \quad 1 \quad 0>$ configuration is maintained under minimization. However, each minimized state is tested to guarantee that a recombination event has not occurred and the cluster still contains $(n+1)$ vacancies. 


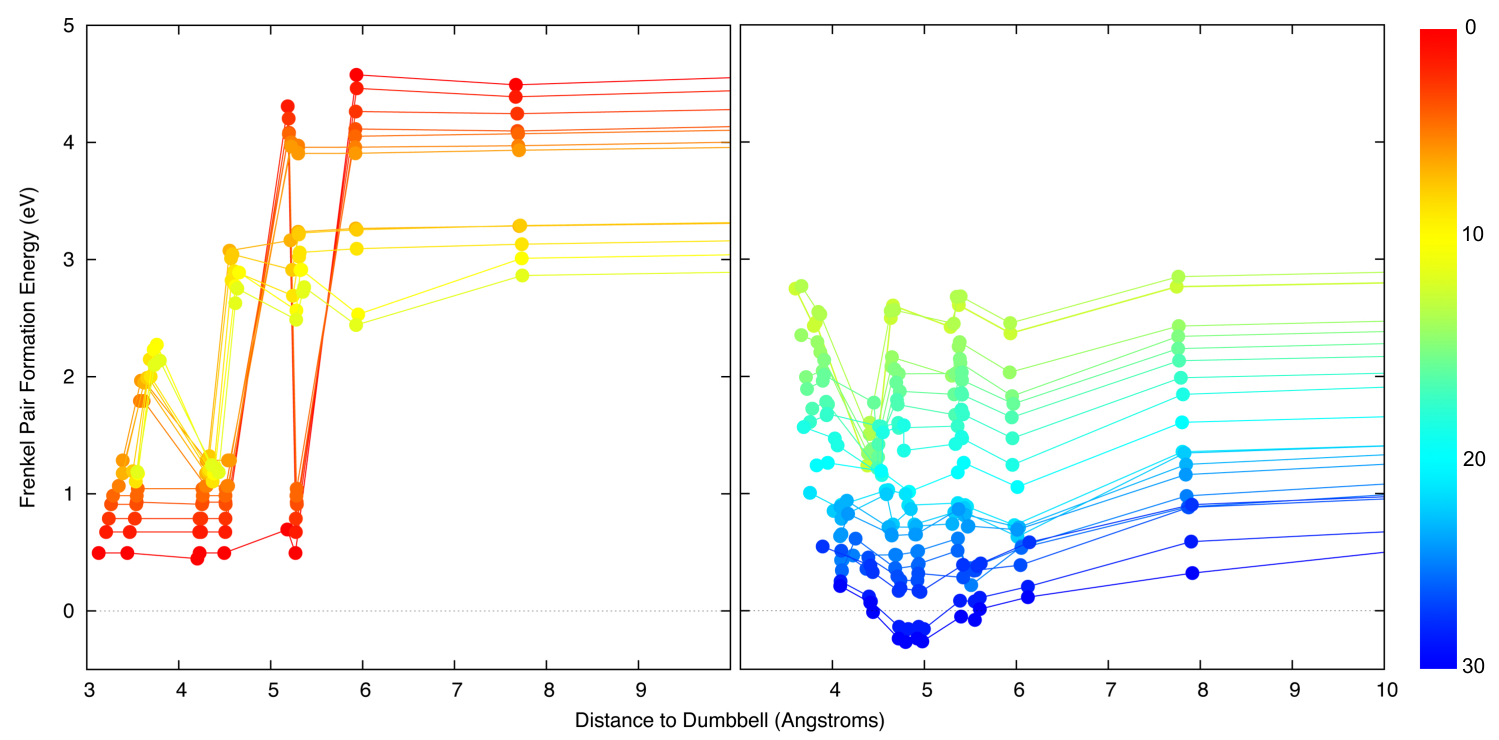

Figure 39: Curves for Frenkel pair formation energy for pure helium bubbles. The $x$-axis shows the distance of the interstitial from the center of the bubble; $y$-axis shows the energy of the system containing the Frenkel pair referenced to the system without it. The curves are broken into two plots for ease of understanding. The red curve shows data for $j=0$; as the curves progress to blue $(j=30)$, the helium concentration increases.

The difference in the energy $\Delta E$ of the $\left(\mathrm{H}_{m} \mathrm{He}_{j} \mathrm{~V}_{n+1}+\mathrm{SIA}\right)$ configuration and the energy of the $\mathrm{H}_{m} \mathrm{He}_{j} \mathrm{~V}_{n}$ configuration is due to the change in "defect status" of the atoms in and around the bubble. This include mainly the Frenkel pair formation energy, but also may be affected by the hydrogen atoms on the edge of the cluster:

$$
\Delta E=E_{t o t}\left(\mathrm{H}_{m} \mathrm{He}_{j} \mathrm{~V}_{n+1}+\mathrm{SIA}\right)-E_{t o t}\left(\mathrm{H}_{m} \mathrm{He}_{j} \mathrm{~V}_{n}\right)
$$

If the value of $\Delta E$ is below zero, it is more energetically favorable for a Frenkel pair to exist than not; therefore, it is more favorable for loop punching to occur. This procedure allows us to examine the energetics of loop punching without the computational expense of performing dynamics and simply waiting to observe the phenomenon.

In Figure 39, several curves for Frenkel pair formation energies in pure helium bubbles $(m=0)$ are shown. The distance shown on the $x$-axis is the distance from the center of the void to the dumbbell; the vacancy component of the Frenkel pair is stationary. For the $n=6$ case, loop punching become energetically favorable when $j \geq 29$; that is, when the ratio of helium to vacancies is $\sim 4.8$. There is a small barrier for this event to occur. 
We perform these same calculations on bubbles containing both $m=15$, with varying amounts of helium, where $n=6$. Representative curves for $j=28,29$ may be seen in Figure 40. As with the pure helium bubbles, as the interstitial dumbbell of the Frenkel pair is moved farther away from the surface of the bubble, the energy of a given system initially drops, but quickly rises again. This indicates that an emitted self-interstitial will be bound to the cluster at a distance of 1-2 $\AA$ from the surface. As the interstitial is moved into the bulk, each curve levels off to some asymptotic value.

Thus we can conclude that loop punching becomes energetically favorable in pure helium bubbles when $j=29$. However, when 15 hydrogen atoms are also included in the cluster, loop punching is favorable with only 27 helium atoms. This is shown in Figure 41, where the minimum value of the $\Delta E$ curve is given for each cluster. In almost all cases, when the concentration of helium is high, the presence of 15 hydrogen atoms in the clusters makes loop punching more favorable than it would otherwise be. This may be explained through the binding of hydrogen atoms. When the concentration of helium is high, some hydrogen is forced outside of the inner radius and may be considered to be occupying an interstitial position in the bulk, as shown in Figure 36. For hydrogen, occupying an interstitial position is less energetically favorable than being within a vacancy. Thus, the system is able to lower its energy by creating a Frenkel pair, removing the hydrogen interstitial by reabsorption into the cluster, and increasing the free surface of the bubble.

\subsection{Discussion}

In our simulations of $\mathrm{H}_{m} \mathrm{He}_{j} \mathrm{~V}_{n}$ clusters, we see that the low energy configuration is a core of helium surrounded by a shell of hydrogen. At high concentrations of helium, the presence of hydrogen makes loop punching more energetically favorable than it would otherwise be. The mechanisms behind the synergistic effects of hydrogen and helium are complicated and not obvious, and there are many variables that may affect the outcome of these calculations, not limited to bubble size, interatomic potentials, and temperature effects. However, our work provides a basis for further study. 


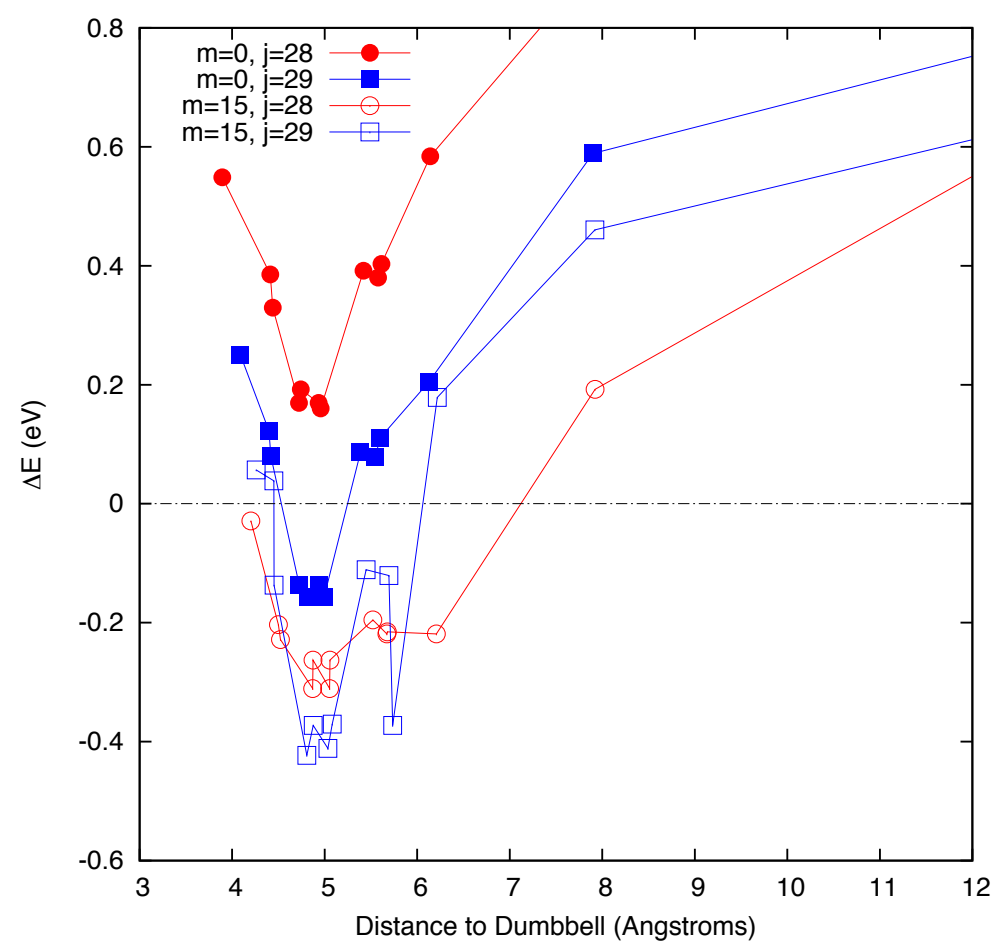

Figure 40: Curves showing the difference in energy between clusters $\left(\mathrm{H}_{m} \mathrm{He}_{j} \mathrm{~V}_{6}\right)$ with and without a Frenkel pair. The $x$-axis shows the distance of the interstitial from the center of the bubble; $y$-axis shows the energy of the system containing the Frenkel pair referenced to the system without it. The red curves with cirucular data points show data for $j=28$; the blue curves with square points show data for $j=29$. Filled data points represent pure helium bubbles; empty data points represent bubbles with 15 hydrogen atoms. It is more energetically favorable for a Frenkel pair to exist when the bubbles contain hydrogen. The SIA is bound to the bubble a short distance from the surface. 


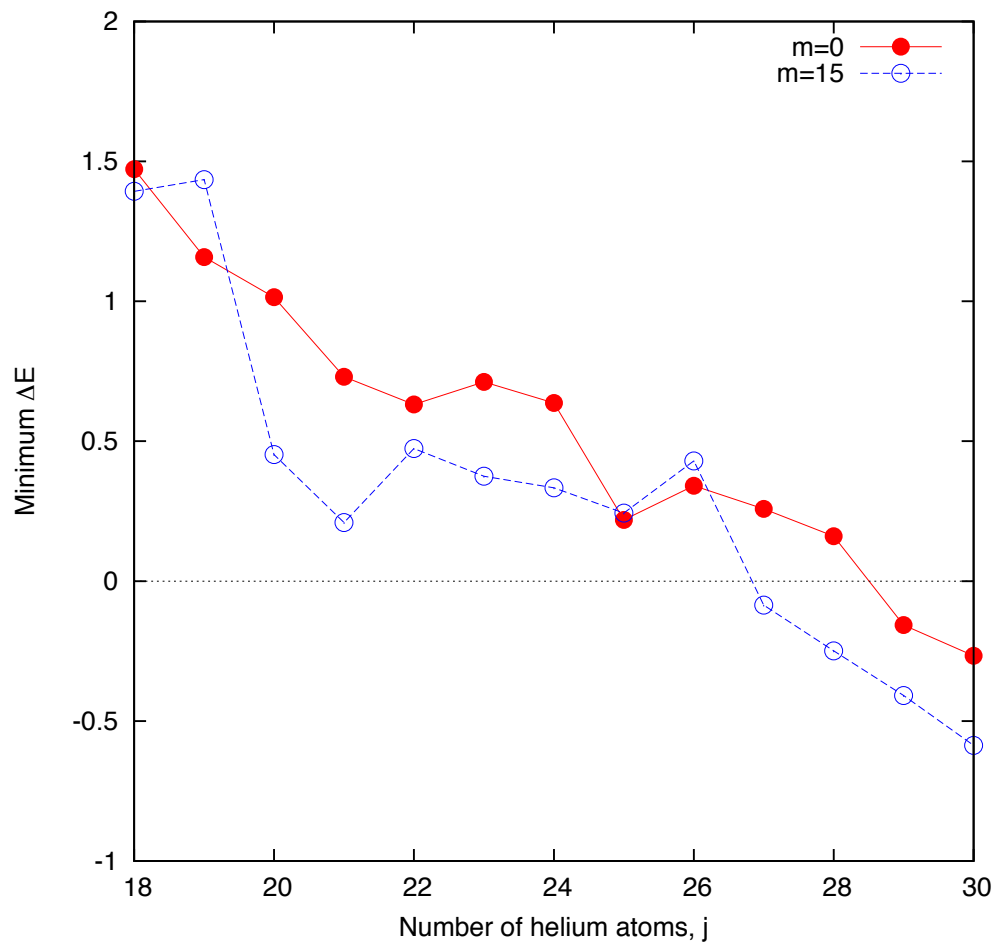

Figure 41: For each $\mathrm{H}_{m} \mathrm{He}_{j} \mathrm{~V}_{6}$ cluster that is tested for energetic the favorability of loop punching, a curve is generated as the interstitial of a Frenkel pair is moved away from the cluster (as shown in Figure 40; this graph shows the minima of those curves. Without hydrogen, loop punching is energetically favorable when $j \geq 29$. Including hydrogen reduces the amount of helium needed to $j=27$. In almost all cases, loop punching is more energetically favorable when hydrogen is included in a cluster. 
It can be argued that the interatomic potentials used in this work do not accurately represent the true interactions, however, we do not feel that any deficiencies in the potentials would substantially change the nature of our conclusions. First, while the H-H interaction potential does provide an attractive well, $\mathrm{H}_{2}$ molecules are not represented entirely realistically by the EAM formalism. It may be argued that hydrogen could be retained in the center of bubbles lacking helium, contrary to our simulations. While this is entirely plausible for large enough bubbles, we capture the most relevant interactions for the analysis of synergistic and radiation effects. It has been shown with ab initio methods [140] that an $\mathrm{H}_{2}$ molecule placed in a vacancy will dissociate and hybridize with iron; this is seen in the covering of the free surfaces of the bubbles with hydrogen [62]. Indeed, if hydrogen were to be contained in molecular form within the bubbles, the pressures inside the bubbles should only increase, strengthening our conclusions.

Second, the interaction potential between hydrogen and helium may change in the presence of iron. However, there is no obvious physical mechanism that would result in a significantly stronger bonding of hydrogen to helium due to the proximity of iron; hydrogen's predilection to hybridize with iron would still presumably be the dominant interaction. Thus, we believe that the character of our main conclusions would not change even if an interatomic potential incorporating all the nuances of the interactions between iron, hydrogen, and helium were used.

However, the area of interatomic potential creation and validation is a very active one, in particular for the iron-helium system $[41,125]$. There are studies which show a dependence on interatomic potentials for clustering behavior [134], so it would be beneficial to explore how our results are affected by the use of different interatomic potentials. 


\section{CHAPTER V}

\section{CONCLUSIONS}

Understanding the interactions between defects in structural materials in a fundamental way is necessary to develop predictive models for irradiated systems. Atomistic simulation is an extremely valuable tool in this pursuit, which allows for the exploration of interactions at a scale and level of detail that is not possible using experiment alone. In this work, we present computational studies of the interactions between point defects and screw line dislocations and the energetics of hydrogen-helium-vacancy clusters in body-centered cubic iron.

In our investigation of dislocation-point defect interactions, we analyze the interaction energy and stability of a variety of interstitial dumbbell configurations and vacancies at a level of detail that has not been investigated before. Comparing our atomistic results to continuum elasticity theory, we provide a guide to where theory is valid and may be safely used in larger scale models, and where more detail that can only be provided by atomistics is required. We observe that a screw dislocation core will spontaneously absorb defects of all types within a few Angstroms, resulting in characteristic core structures.

Next, in our simulations of small gas bubbles in the bulk, we provide a method for searching for minimum energy configurations of hydrogen and helium within vacancy clusters. This method is shown to be of value in our study of multiple hydrogen atoms trapped at monovacancies, in which we find new low energy configurations that have not been studied in the past, and we verify these structures with the help of ab initio methods.

We show that hydrogen has a stabilizing effect on vacancy clusters, causing vacancies to be more tightly bound to clusters, while neighboring iron atoms are less tightly bound. These studies of the detailed energetics of clusters are conducted on bubbles that are larger

than have been studied in the past, while still retaining the detail of individual interatomic interactions.

Finally, we present the first atomistic computational studies of hydrogen-helium-vacancy 
clusters in bcc iron. To accomplish this, we introduce a new interatomic potential for hydrogen and helium. We show that the low energy configuration of a cluster is a core of helium surrounded by a shell of hydrogen. The binding energy of hydrogen, helium, and point defects to the clusters are determined, and we show that the binding of hydrogen is strongly affected by the presence of helium. Finally, we show that the synergistic effects of hydrogen and helium may be explained through the loop punching phenomenon. In this process, the presence of hydrogen makes it more energetically favorable for bubbles to grow through creation of Frenkel pairs at a smaller helium concentration than when hydrogen is absent. The hydrogen atoms are able to be retained more readily by helium bubbles with large free surfaces.

Although we did not perform simulations directly involving both dislocations and hydrogen, further studies linking these phenomena would be beneficial. In the HELP theory of hydrogen embrittlement [10], hydrogen causes dislocations to be mobile, resulting in increased plasticity. We show that the presence of hydrogen does cause self-interstitials to become less well bound to their lattice sites; we also show that the absorption of interstitials leads to rearrangement of the core of a dislocation, resulting in motion of the dislocation. Thus, the methods described in this work could easily be extended to further study these effects.

The interactions of defects within a microstructure are extremely complex and no one atomistic study can fully explain macroscopic behavior. However, our work provides a unique insight into fundamental behavior, as well as providing valuable data that may be used to parameterize further work. 


\section{REFERENCES}

[1] Abramov, E. and Eliezer, D., "Trapping of hydrogen in helium-implanted metals," Journal of Materials Science Letters, vol. 7, no. 2, pp. 108-110, 1988.

[2] Ackland, G., Bacon, D., Calder, A., and Harry, T., "Computer simulation of point defect properties in dilute $\mathrm{Fe}-\mathrm{Cu}$ alloy using a many-body interatomic potential," Philosophical Magazine A, vol. 75, pp. 713-732, Jan 1997.

[3] Ackland, G., Mendelev, M., Srolovitz, D., Han, S., and Barashev, A., "Development of an interatomic potential for phosphorus impurities in alpha-iron," Journal of Physics: Condensed Matter, vol. 16, pp. 2629-2642, 2004.

[4] Azevedo, C., "A review on neutron-irradiation-induced hardening of metallic components," Engineering Failure Analysis, vol. 18, pp. 1921-42, 2011.

[5] Bacon, D., Osetsky, Y., and Rong, Z., "Computer simulation of reactions between an edge dislocation and glissile self-interstitial clusters in iron," Philosophical Magazine, vol. 86, pp. 3921-3936, Jan 2006.

[6] Beck, D., "A new interatomic potential function for helium," Molecular Physics, vol. 14, no. 4, pp. 311-315, 1968.

[7] Besenbacher, F., Bøttiger, J., and Myers, S., "Deuterium trapping in heliumimplanted nickel," Journal of Applied Physics, vol. 53, no. 5, pp. 3547-3551, 1982.

[8] Besenbacher, F., Myers, S., Nordlander, P., and Nørskov, J., "Multiple hydrogen occupancy of vacancies in Fe," Journal of Applied Physics, vol. 61, p. 1788, 1987.

[9] Bhattacharya, A. and Anderson, J., "Exact quantum Monte Carlo calculation of the H-He interaction potential," Physical Review A, vol. 49, no. 4, pp. 2441-2445, 1994.

[10] Birnbaum, H. and Sofronis, P., "Hydrogen-enhanced localized plasticity-a mechanism for hydrogen-related fracture," Materials Science and Engineering: A, vol. 176, no. 1-2, pp. 191-202, 1994.

[11] BlÖchl, P., "Projector augmented-wave method," Physical Review B, vol. 50, pp. 17953-17979, Jan 1994.

[12] Bloom, E., Zinkle, S., and Wiffen, F., "Materials to deliver the promise of fusion power - progress and challenges," Journal of Nuclear Materials, vol. 329, pp. 12-19, 2004 .

[13] Bulatov, V. and Cai, W., Computer simulations of dislocations. Oxford University Press, 2006. 
[14] Burke, K. and Ernzerhof, M., "Generalized gradient approximation made simple," Physical Review Letters, vol. 77, pp. 3865-3868, Jan 1996.

[15] Cai, W., Arsenlis, A., Weinberger, C., and Bulatov, V., "A non-singular continuum theory of dislocations," Journal of the Mechanics and Physics of Solids, vol. 54, pp. 561-587, Jan 2006.

[16] Capolungo, L., Beyerlein, I. J., and Wang, Z. Q., "The role of elastic anisotropy on plasticity in hcp metals: a three-dimensional dislocation dynamics study," Modelling and Simulation in Materials Science and Engineering, vol. 18, no. 085002, 2010.

[17] Caro, A., Hetherly, J., Stukowski, A., Caro, M., Martinez, E., SrivilLiputhur, S., Zepeda-Ruiz, L., and Nastasi, M., "Properties of helium bubbles in Fe and FeCr alloys," Journal of Nuclear Materials, vol. 418, pp. 261-268, 2011.

[18] Castellote, M., Fullea, J., de Viedma, P., Andrade, C., Alonso, C., Llorente, I., Turrillas, X., Campo, J., Schweitzer, J., and Spillane, T., "Hydrogen embrittlement of high-strength steel submitted to slow strain rate testing studied by nuclear resonance reaction analysis and neutron diffraction," Nuclear Instruments and Methods in Physics Research Section B: Beam Interactions with Materials and Atoms, vol. 259, no. 2, pp. 975-983, 2007.

[19] Chang, J., Cai, W., Bulatov, V., and Yip, S., "Molecular dynamics simulations of motion of edge and screw dislocations in a metal," Computational Materials Science, vol. 23, pp. 111-115, Jan 2002.

[20] Chaussidon, J., Fivel, M., and Rodney, D., "The glide of screw dislocations in bcc Fe: atomistic static and dynamic simulations," Acta Materialia, vol. 54, no. 13, pp. 3407-3416, 2006.

[21] Chen, J., Hoffelner, W., Ullmaier, H., and Jung, P., "Dislocation loops and bubbles in oxide dispersion strengthened ferritic steel after helium implantation under stress," Acta Materialia, vol. 56, pp. 250-258, 2008.

[22] Chiesa, S., Gilbert, M., Dudarev, S., Derlet, P., and Swygenhoven, H. V., "The non-degenerate core structure of a $1 / 2<111>$ screw dislocation in bcc transition metals modelled using Finnis-Sinclair potentials: The necessary and sufficient conditions," Philosophical Magazine, vol. 89, no. 34, pp. 3235-3243, 2009.

[23] Clouet, E., Garruchet, S., Nguyen, H., Perez, M., and Becquart, C., "Dislocation interaction with $\mathrm{C}$ in $\alpha$-Fe: A comparison between atomic simulations and elasticity theory," Acta Materialia, vol. 56, no. 14, pp. 3450-3460, 2008.

[24] Condon, J. and Schober, T., "Hydrogen bubbles in metals," Journal of Nuclear Materials, vol. 207, pp. 1-24, 1993.

[25] Daw, M. and Baskes, M., "Semiempirical, quantum-mechanical calculation of hydrogren embrittlement in metals," Physical Review Letters, vol. 50, pp. 1285-1288, Jan 1983.

[26] Daw, M. and Baskes, M., "Embedded-atom method: Derivation and application to impurities, surfaces, and other defects in metals," Physical Review B, vol. 29, pp. 6443-6453, Jan 1984. 
[27] Daw, M., Folles, S., and Baskes, M., "The embedded-atom method - a review of theory and applications," Materials Science Reports, vol. 9, pp. 251-310, Jan 1993.

[28] Dederichs, P. and Schroeder, K., "Anisotropic diffusion in stress fields," Physical Review B, vol. 17, no. 6, p. 2524, 1978.

[29] Deo, C., Okuniewski, M., Srivilliputhur, S., Maloy, S., Baskes, M., James, M., and Stubbins, J., "Helium bubble nucleation in bcc iron studied by kinetic Monte Carlo simulations," Journal of Nuclear Materials, vol. 361, no. 2-3, pp. 141$148,2007$.

[30] Domain, C. and Monnet, G., "Simulation of screw dislocation motion in iron by molecular dynamics simulations," Physical Review Letters, vol. 95, p. 215506, Nov 2005 .

[31] Dudarev, S. and Derlet, P., "A 'magnetic' interatomic potential for molecular dynamics simulations," Journal of Physics: Condensed Matter, vol. 17, pp. 70977118 , Oct 2005.

[32] Duesbery, M. and Vitek, V., "Plastic anisotropy in bcc transition metals," Acta Materialia, vol. 46, no. 5, pp. 1481-1492, 1998.

[33] Duesbery, M., Vitek, V., and Bowen, D., "Effect of shear-stress on screw dislocation core structure in body-centered cubic lattices," Proceedings of the Royal Society of London A, vol. 332, pp. 85-111, Jan 1973.

[34] Eshelby, J., Read, W., and Shockley, W., "Anisotropic elasticity with applications to dislocation theory," Acta Metallurgica, vol. 1, no. 3, pp. 251-259, 1953.

[35] Evans, J., "The role of implanted gas and lateral stress in blister formation mechanisms," Journal of Nuclear Materials, vol. 76 \& 77, pp. 228-234, Jan 1978.

[36] Finnis, M. and Sinclair, J., "A simple empirical n-body potential for transition metals," Philosophical Magazine A, vol. 50, no. 1, pp. 45-55, 1984.

[37] Frederiksen, S. and Jacobsen, K., "Density functional theory studies of screw dislocation core structures in bcc metals," Philosophical Magazine, vol. 83, pp. 365375, Jan 2003.

[38] Fu, C.-C. and Willaime, F., "Ab initio study of helium in alpha-Fe: Dissolution, migration, and clustering with vacancies," Physical Review B, vol. 72, no. 6, p. 064117, 2005.

[39] Fujita, S., Okita, T., Kuramoto, E., and Sekimura, N., "A study of the interaction between irradiation induced-defect and a line dislocation in bcc-iron," Journal of Nuclear Materials, vol. 386-388, pp. 93-96, Feb 2009.

[40] FukAI, Y., The Metal-Hydrogen System. Springer, 2005.

[41] Gao, N., Samaras, M., and Swygenhoven, H. V., "A new Fe-He pair potential," Journal of Nuclear Materials, vol. 400, pp. 240-244, 2010. 
[42] Gao, N., Swygenhoven, H. V., Victoria, M., and Chen, J., "Formation of dislocation loops during He clustering in bcc Fe," Journal of Physics: Condensed Matter, vol. 23, p. 442201, 2011.

[43] Gao, N., Victoria, M., Chen, J., and Swygenhoven, H. V., "Helium-vacancy cluster in a single bcc iron crystal lattice," Journal of Physics: Condensed Matter, vol. 23, p. 245403, 2011.

[44] Garner, F. A., Oliver, B., Greenwood, L., James, M., Ferguson, P., Maloy, S., and Sommer, W., "Determination of helium and hydrogen yield from measurements on pure metals and alloys irradiated by mixed high energy proton and spallation neutron spectra in lansce," Journal of Nuclear Materials, vol. 296, no. 1-3, pp. $66-82,2001$.

[45] Garner, F. A., Simonen, E., Oliver, B., Greenwood, L., Grossbeck, M., Wolfer, W., and Scott, P., "Retention of hydrogen in fcc metals irradiated at temperatures leading to high densities of bubbles or voids," Journal of Nuclear Materials, vol. 356, no. 1-3, pp. 122-135, 2006.

[46] Ghoniem, N., Tong, S., Huang, J., Singh, B., and Wen, M., "Mechanisms of dislocation-defect interactions in irradiated metals investigated by computer simulations," Journal of Nuclear Materials, vol. 307, pp. 843-851, 2002.

[47] Gillan, M., "The elastic dipole tensor for point defects in ionic crystals," Journal of Physics C: Solid State Physics, vol. 17, no. 9, pp. 1473-1488, 1984.

[48] Gordon, P., Neeraj, T., Li, Y., and Li, J., "Screw dislocation mobility in bcc metals: the role of the compact core on double-kink nucleation," Modelling and Simulation in Materials Science and Engineering, vol. 18, p. 085008, 2010.

[49] Greenwood, G., Foreman, A., and Rimmer, D., "The role of vacancies and dislocations in the nucleation and growth of gas bubbles in irradiated fissile material," Journal of Nuclear Materials, vol. 4, pp. 305-324, 1959.

[50] Gröger, R., Racherla, V., Bassani, J., and Vitek, V., "Multiscale modeling of plastic deformation of molybdenum and tungsten: II. yield criterion for single crystals based on atomistic studies of glide of $1 / 2<111>$ screw dislocations," Acta Materialia, vol. 56, no. 19, pp. 5412-5425, 2008.

[51] Gröger, R. and Vitek, V., "Directional versus central-force bonding in studies of the structure and glide of $1 / 2<111>$ screw dislocations in bcc transition metals," Philosophical Magazine, vol. 89, no. 34-36, pp. 3163-3178, 2009.

[52] Groh, S. and ZBIB, H., "Advances in discrete dislocations dynamics and multiscale modeling," Journal of Engineering Materials and Technology, vol. 131, p. 041209, 2009.

[53] Haghighat, S., Lucas, G., and Schaublin, R., "State of a pressurized helium bubble in iron," Europhysics Letters, vol. 85, p. 60008, 2009.

[54] Hayward, E. and Deo, C., "Finding minimum energy states of hydrogen-vacancy clusters in iron," Transactions of the American Nuclear Society, vol. 104, pp. 63-64, Apr 2011. 
[55] Hayward, E. and Deo, C., "Energetics of small hydrogen-vacancy clusters in bcc iron," Journal of Physics: Condensed Matter, vol. 23, p. 425402, Oct 2011.

[56] Head, A., "The [111] dislocation in a cubic crystal," physica status solidi (b), vol. 6, no. 2, pp. 461-465, 1964.

[57] Heinisch, H., Gao, F., and Kurtz, R., "Atomic-scale modeling of interactions of helium, vacancies and helium-vacancy clusters with screw dislocations in alpha-iron," Philosophical Magazine, vol. 90, no. 7, pp. 885-895, 2010.

[58] Hirth, J., "Effects of hydrogen on the properties of iron and steel," Metallurgical and Materials Transactions A, vol. 11, no. 6, pp. 861-890, 1980.

[59] Hirth, J. and Gehlen, P., "Dislocation displacement fields in anisotropic media," Journal of Applied Physics, vol. 40, no. 5, pp. 2177-2181, 1969.

[60] Hirth, J. and Lothe, J., Theory of Dislocations. McGraw-Hill, Inc., 1982.

[61] Hohenberg, P. and Kohn, W., "Inhomogeneous electron gas," Physical Review, vol. 136, pp. B864-B871, Jan 1964.

[62] Irigoyen, B., Ferullo, R., Castellani, N., and Juan, A., "The interaction of hydrogen with an Fe vacancy: a molecular orbital simulation," Journal of Physics D: Applied Physics, vol. 29, pp. 1306-1309, 1996.

[63] Iwamoto, M. and Fukai, Y., "Superabundant vacancy formation in iron under high hydrogen pressures: Thermal desorption spectroscopy," Materials Transactions-JIM, vol. 40, no. 7, pp. 606-611, 1999.

[64] Iyer, R. and Pickering, H., "Mechanism and kinetics of electrochemical hydrogen entry and degradation of metallic systems," Annual Review of Materials Science, vol. 20, no. 1, pp. 299-338, 1990.

[65] Jepsen, O. and Andersen, O., "Improved tetrahedron method for brillouin-zone integrations," Physical Review B, vol. 49, pp. 16223-16233, Jan 1994.

[66] Jiang, D. and Carter, E. A., "Diffusion of interstitial hydrogen into and through bcc Fe from first principles," Physical Review B, vol. 70, no. 6, p. 64102, 2004.

[67] Joubert, D., "From ultrasoft pseudopotentials to the projector augmented-wave method," Physical Review B, vol. 59, pp. 1758-1775, Jan 1999.

[68] Juan, A., Irigoyen, B., and Gesari, S., "Electronic structure and bonding of hydrogen in a screw dislocated bcc Fe," Applied Surface Science, vol. 172, pp. 8-17, Jan 2001.

[69] Juslin, N. and Nordlund, K., "Pair potential for Fe-He," Journal of Nuclear Materials, vol. 382, no. 2-3, pp. 143-146, 2008.

[70] Kohn, W. and Sham, L., "Self-consistent equations including exchange and correlation effects," Physical Review, vol. 140, pp. A1133-A1138, Jan 1965.

[71] Kresse, G., "Efficient iterative schemes for ab initio total-energy calculations using a plane-wave basis set," Physical Review B, vol. 54, pp. 11169-11186, Jan 1996. 
[72] Krom, A. and BAKker, A., "Hydrogen trapping models in steel," Metallurgical and Materials Transactions B, vol. 31, no. 6, pp. 1475-1482, 2000.

[73] Kurtz, R., Heinisch, H., and GaO, F., "Modeling of He-defect interactions in ferritic alloys for fusion," Journal of Nuclear Materials, vol. 382, no. 2-3, pp. 134-142, 2008.

[74] Lennard-Jones, J., "Cohesion," Proceedings of the Physical Society, vol. 43, p. 461, 1931.

[75] LiU, X. and Biner, S., "Molecular dynamics simulations of the interactions between screw dislocations and self-interstitial clusters in body-centered cubic Fe," Scripta Materialia, vol. 59, no. 1, pp. 51-54, 2008.

[76] LiU, Z. L., Liu, X. M., Zhuang, Z., and You, X. C., "A multi-scale computational model of crystal plasticity at submicron-to-nanometer scales," International Journal of Plasticity, vol. 25, pp. 1436-1455, Aug 2009.

[77] Lucas, G. and Schäublin, R., "Stability of helium bubbles in alpha-iron: A molecular dynamics study," Journal of Nuclear Materials, vol. 386, pp. 360-362, 2009.

[78] Masuda, K., Sugano, K., and Sato, A., "Interaction energy between a selfinterstitial and a $(1 / 2)<111>$ screw dislocation in b. c. c. transition metals," Journal of the Physical Society of Japan, vol. 48, no. 4, pp. 1233-1236, 1980.

[79] Masuda-Jindo, K., "On the interaction between a screw dislocation and point defects in bcc transition metals," physica status solidi (b), vol. 129, no. 2, pp. 595-599, 1985.

[80] McDowell, D., "Viscoplasticity of heterogeneous metallic materials," Materials Science and Engineering: R: Reports, vol. 62, no. 3, pp. 67-123, 2008.

[81] Mclellan, R. and Harkins, C., "Hydrogen interactions with metals," Mater Sci Eng, vol. 18, pp. 5-35, 1975.

[82] Meissner, N., Savino, E., Willis, J., and Bullough, R., "The dislocation loop in an anisotropic medium and its interaction with an interstitial atom," physica status solidi (b), vol. 63, no. 1, 1974.

[83] Mendelev, M., Han, S., Srolovitz, D., Ackland, G., Sun, D., and Asta, M., "Development of new interatomic potentials appropriate for crystalline and liquid iron," Philosophical Magazine, vol. 83, no. 35, pp. 3977-3994, 2003.

[84] Methfessel, M., "High-precision sampling for brillouin-zone integration in metals," Physical Review B, vol. 40, pp. 3616-3621, Jan 1989.

[85] Metropolis, N., Rosenbluth, A., Rosenbluth, M., Teller, A., and Teller, E., "Equation of state calculations by fast computing machines," The Journal of Chemical Physics, vol. 21, no. 6, p. 1087, 1953.

[86] MilleR, W., Treatise on Crystallography. Cambridge, 1839.

[87] Monasterio, P., Lau, T., Yip, S., and Vliet, K. V., "Hydrogen-vacancy interactions in Fe-C alloys," Physical Review Letters, vol. 103, no. 8, p. 85501, 2009. 
[88] Monti, A., Sarce, A., Smetniansky-DeGrande, N., Savino, E., and Tomé, C., "Point defects and sink strength in h.c.p. metals," Philosophical Magazine A, vol. 63, pp. 925-936, Jan 1991.

[89] Moriarty, J., Vitek, V., Bulatov, V., and Yip, S., "Atomistic simulations of dislocations and defects," Journal of Computer-Aided Materials Design, vol. 9, pp. 99132, Jan 2002.

[90] Morishita, K., "Nucleation path of helium bubbles in metals during irradiation," Philosophical Magazine, vol. 87, no. 7, pp. 1139-1158, 2007.

[91] Morishita, K., Sugano, R., and Wirth, B. D., "MD and KMC modeling of the growth and shrinkage mechanisms of helium-vacancy clusters in Fe," Journal of Nuclear Materials, vol. 323, no. 2-3, pp. 243-250, 2003.

[92] Morishita, K., Sugano, R., Wirth, B. D., and de La Rubia, T. D., "Thermal stability of helium-vacancy clusters in iron," Nuclear Instruments and Methods in Physics Research, B, vol. 202, pp. 76-81, 2003.

[93] Mrovec, M., Nguyen-Manh, D., Elsässer, C., and Gumbsch, P., "Magnetic bond-order potential for iron," Physical Review Letters, vol. 106, no. 24, p. 246402, 2011.

[94] Mughrabi, H., "On the current understanding of strain gradient plasticity," Materials Science and Engineering: A, vol. 387, pp. 209-213, 2004.

[95] Muller, M., Erhart, P., and Albe, K., "Analytic bond-order potential for bcc and fcc iron-comparison with established embedded-atom method potentials," Journal of Physics: Condensed Matter, vol. 19, no. 32, p. 326220, 2007.

[96] Myers, S., Baskes, M., Birnbaum, H., Corbett, J., Deleo, G., Estreicher, S., Haller, E., Jena, P., Johnson, N., and Kirchheim, R., "Hydrogen interactions with defects in crystalline solids," Reviews of Modern Physics, vol. 64, no. 2, pp. 559-617, 1992.

[97] Myers, S., Follstaedt, D., Besenbacher, F., and Bøttiger, J., "Trapping and surface permeation of deuterium in He-implanted Fe," Journal of Applied Physics, vol. 53, no. 12, pp. 8734-8744, 1982.

[98] Myers, S., Picraux, S., and Stoltz, R., "Defect trapping of ion-implanted deuterium in Fe," Journal of Applied Physics, vol. 50, p. 5710, 1979.

[99] Myers, S., Richards, P., Wampler, W., and Besenbacher, F., "Ion-beam studies of hydrogen-metal interactions," Journal of Nuclear Materials, vol. 165, no. 1, pp. 9-64, 1989.

[100] Nabarro, F., "Theory of crystal dislocations," Oxford University Press, 1967.

[101] Nagumo, M., "Function of hydrogen in embrittlement of high-strength steels," ISIJ International, vol. 41, pp. 590-598, Jan 2001.

[102] Nagumo, M., Nakamura, M., and Takai, K., "Hydrogen thermal desorption relevant to delayed-fracture susceptibility of high-strength steels," Metallurgical and Materials Transactions A, vol. 32, no. 2, pp. 339-347, 2001. 
[103] Nordlander, P., Nørskov, J., Besenbacher, F., and Myers, S., "Multiple deuterium occupancy of vacancies in Pd and related metals," Physical Review B, vol. 40, no. 3, pp. 1990-1992, 1989.

[104] NøRskov, J., "Covalent effects in the effective-medium theory of chemical binding: Hydrogen heats of solution in the 3d metals," Physical Review B, vol. 26, no. 6, pp. 2875-2885, 1982.

[105] Nørskov, J. and Besenbacher, F., "Theory of hydrogen interaction with metals," Journal of the Less Common Metals, vol. 130, pp. 475-490, 1987.

[106] Oriani, R., "The diffusion and trapping of hydrogen in steel," Acta Metallurgica, vol. 18, no. 1, pp. 147-157, 1970.

[107] Oriani, R., "Hydrogen embrittlement of steels," Annual Review of Materials Science, vol. 8, no. 1, pp. 327-357, 1978.

[108] Oriani, R. and Josephic, P., "Equilibrium aspects of hydrogen-induced cracking of steels," Acta Metallurgica, vol. 22, no. 9, pp. 1065-1074, 1974.

[109] Oriani, R. and Josephic, P., "Equilibrium and kinetic studies of the hydrogenassisted cracking of steel," Acta Metallurgica, vol. 25, no. 9, pp. 979-988, 1977.

[110] Osetsky, Y., Bacon, D., Serra, A., Singh, B., and Golubov, S., "Stability and mobility of defect clusters and dislocation loops in metals," Journal of Nuclear Materials, vol. 276, no. 1, pp. 65-77, 2000.

[111] Osetsky, Y., Serra, A., and Priego, V., "Interactions between mobile dislocation loops in Cu and $\alpha$-fe," Journal of Nuclear Materials, vol. 276, no. 1, pp. 202-212, 2000.

[112] Patra, A. and McDowell, D., "Crystal plasticity-based constitutive modelling of irradiated bcc structures," Philosophical Magazine, pp. 1-27, Jan 2011.

[113] Plimpton, S., "Fast parallel algorithms for short-range molecular dynamics," Journal of Computational Physics, vol. 117, no. 1, pp. 1-19, 1995.

[114] Pronsato, M., Brizuela, G., and Juan, A., "The electronic structure and location of H pairs in bcc Fe edge dislocation," Applied Surface Science, vol. 173, pp. 368-379, Jan 2001.

[115] Pronsato, M., Pistonesi, C., and Juan, A., "Density functional study of H-Fe vacancy interaction in bcc iron," Journal of Physics: Condensed Matter, vol. 16, p. $6907,2004$.

[116] Puska, M. and Nieminen, R., "Theory of hydrogen and helium impurities in metals," Physical Review B, vol. 29, no. 10, pp. 5382-5397, 1984.

[117] QIn, Q. and Bassani, J., "Non-associated plastic flow in single crystals," Journal of the Mechanics and Physics of Solids, vol. 40, no. 4, pp. 835-862, 1992.

[118] Racherla, V. and Bassani, J., "Strain burst phenomena in the necking of a sheet that deforms by non-associated plastic flow," Modelling and Simulation in Materials Science and Engineering, vol. 15, p. S297, 2007. 
[119] Ramasubramaniam, A., Itakura, M., and Carter, E. A., "Interatomic potentials for hydrogen in alpha-iron based on density functional theory," Physical Review $B$, vol. 79 , no. 17 , p. $174101,2009$.

[120] Ramasubramaniam, A., Itakura, M., Ortiz, M., and Carter, E. A., "Effect of atomic scale plasticity on hydrogen diffusion in iron: Quantum mechanically informed and on-the-fly kinetic monte carlo simulations," Journal of Materials Research, vol. 23, pp. 2757-2773, Jan 2008.

[121] Ramasubramaniam, A., Itakura, M., and Carter, E. A., "Erratum: Interatomic potentials for hydrogen in alpha-iron based on density functional theory [Phys. Rev. B 79, 174101 (2009)]," Physical Review B, vol. 81, p. 099902, Mar 2010.

[122] Rhee, M., Stolken, J., Bulatov, V., de la Rubia, T., Zbib, H., and Hirth, J., "Dislocation stress fields for dynamic codes using anisotropic elasticity: methodology and analysis," Materials Science and Engineering: A, vol. 309, pp. 288-293, 2001.

[123] Schaublin, R. and Baluc, N., "Radiation damage in ferritic/martensitic steels for fusion reactors: a simulation," Nuclear Fusion, vol. 47, pp. 1690-1695, 2007.

[124] Sekimura, N., Iwai, T., Arai, Y., Yonamine, S., Naito, A., Miwa, Y., and HAMADA, S., "Synergistic effects of hydrogen and helium on microstructural evolution in vanadium alloys by triple ion beam irradiation," Journal of Nuclear Materials, vol. 283, pp. 224-228, 2000.

[125] Seletskaia, T., Osetsky, Y., Stoller, R., and Stocks, G., "Development of a Fe-He interatomic potential based on electronic structure calculations," Journal of Nuclear Materials, vol. 367, pp. 355-360, 2007.

[126] Seletskaia, T., Osetsky, Y., Stoller, R., and Stocks, G., "Calculation of helium defect clustering properties in iron using a multi-scale approach," Journal of Nuclear Materials, vol. 351, no. 1-3, pp. 109-118, 2006.

[127] Shastry, V. and De La Rubia, T. D., "The interaction between point defects and edge dislocation in bcc iron," Journal of Engineering Materials and Technology, vol. 121, p. 126, 1999.

[128] Sivak, A., Chernov, V., Dubasova, N., and Romanov, V., "Anisotropy migration of self-point defects in dislocation stress fields in bcc Fe and fcc Cu," Journal of Nuclear Materials, vol. 367, pp. 316-321, Jan 2007.

[129] Sivak, A., Romanov, V., and Chernov, V., "Diffusion of self-point defects in body-centered cubic iron crystal containing dislocations," Crystallography Reports, vol. 55, pp. 97-108, Jan 2010.

[130] Smetniansky-DeGrande, N., Savino, E., and C.N.Tome, "Stress induced anisotropic diffusion of intrinsic point defects towards dislocations in h.c.p. crystals," physica status solidi (b), vol. 144, p. 271, 1987.

[131] Song, J. and Curtin, W. A., "A nanoscale mechanism of hydrogen embrittlement in metals," Acta Materialia, vol. 59, pp. 1557-1569, Feb 2011. 
[132] Sørensen, M. and Voter, A., "Temperature-accelerated dynamics for simulation of infrequent events," The Journal of Chemical Physics, vol. 112, p. 9599, 2000.

[133] Stewart, D. and Osetsky, Y., "Atomistic studies of formation and diffusion of helium clusters and bubbles in bcc iron," Journal of Nuclear Materials, Jan 2010.

[134] Stewart, D., Osetsky, Y., Stoller, R., Golubov, S., Seletskaia, T., and Kamenski, P., "Atomistic studies of helium defect properties in bcc iron: comparison of He-Fe potentials," Philosophical Magazine, vol. 90, no. 7-8, pp. 935-944, 2010.

[135] Stoller, R., Golubov, S., Kamenski, P., Seletskaia, T., and Osetsky, Y., "Implementation of a Fe-He 3-body interatomic potential," Oak Ridge National Lab Report (unpublished).

[136] Stroh, A. N., "Dislocations and cracks in anisotropic elasticity," Philosophical Magazine, vol. 3, no. 625, 1958.

[137] Taketomi, S., Matsumoto, R., and Miyazaki, N., "Atomistic study of hydrogen distribution and diffusion around a $\left\{\begin{array}{lll}1 & 1 & 2\end{array}\right\}<\begin{array}{lll}1 & 1 & 1>\text { edge dislocation in alpha iron," }\end{array}$ Acta Materialia, vol. 56, no. 15, pp. 3761-3769, 2008.

[138] Tanaka, T., Oka, K., Ohnuki, S., Yamashita, S., Suda, T., Watanabe, S., and WAKAI, E., "Synergistic effect of helium and hydrogen for defect evolution under multi-ion irradiation of Fe-Cr ferritic alloys," Journal of Nuclear Materials, vol. 329, pp. 294-298, 2004.

[139] Tanguy, D. and Mareschal, M., "Superabundant vacancies in a metal-hydrogen system: Monte carlo simulations," Physical Review B, vol. 72, no. 17, p. 174116, 2005.

[140] Tateyama, Y. and Ohno, T., "Atomic-scale effects of hydrogen in iron toward hydrogen embrittlement: Ab-initio study," ISIJ international, vol. 43, no. 4, pp. 573$578,2003$.

[141] Tateyama, Y. and Ohno, T., "Stability and clusterization of hydrogen-vacancy complexes in alpha-fe: An ab initio study," Physical Review B, vol. 67, p. 174105, May 2003.

[142] Tolstolutskaya, G., Ruzhytskiy, V., Kopanets, I., Karpov, S., Bryk, V., Voyevodin, V., and Garner, F. A., "Displacement and helium-induced enhancement of hydrogen and deuterium retention in ion-irradiated $18 \mathrm{Cr} 10 \mathrm{NiTi}$ stainless steel," Journal of Nuclear Materials, vol. 356, no. 1-3, pp. 136-147, 2006.

[143] Tomé, C., Cecatto, H., and Savino, E., "Point-defect diffusion in a strained crystal," Physical Review B, vol. 25, no. 12, pp. 7428-7440, 1982.

[144] Tomé, C. and Savino, E., "Interaction between point defects and straight dislocations in hexagonal crystals," Materials Science and Engineering, vol. 24, pp. 109-122, Jan 1976.

[145] Trinkaus, H. and Singh, B., "Helium accumulation in metals during irradiationwhere do we stand?," Journal of Nuclear Materials, vol. 323, pp. 229-242, 2003. 
[146] Trinkaus, H. and Wolfer, W., "Conditions for dislocation loop punching by helium bubbles," Journal of Nuclear Materials, vol. 122 \& 123, pp. 552-557, Jan 1984.

[147] Troiano, A. R., "The role of hydrogen and other interstitials in the mechanical behavior of metals," Trans. ASM, vol. 52, p. 54, 1960.

[148] Ventelon, L. and Willaime, F., "Core structure and Peierls potential of screw dislocations in $\alpha$-Fe from first principles: cluster versus dipole approaches," Journal of Computer-Aided Materials Design, vol. 14, pp. 85-94, 2007.

[149] Ventelon, L. and Willaime, F., "Generalized stacking-faults and screw-dislocation core-structure in bcc iron: A comparison between ab initio calculations and empirical potentials," Philosophical Magazine, vol. 90, pp. 1063-1074, Mar 2010.

[150] Vitek, V., "Computer simulation of the screw dislocation motion in b.c.c. metals under the effect of the external shear and uniaxial stresses," Proceedings of the Royal Society of London A, vol. 352, pp. 109-124, Jan 1976.

[151] Vitek, V., "Structure of dislocation cores in metallic materials and its impact on their plastic behavior," Progress in Materials Science, vol. 36, pp. 1-27, Jan 1992.

[152] Wakai, E., Sawai, T., Furuya, K., Naito, A., Aruga, T., Kikuchi, K., Yamashita, S., Ohnuki, S., Yamamoto, S., and Naramoto, H., "Effect of triple ion beams in ferritic/martensitic steel on swelling behavior," Journal of Nuclear Materials, vol. 307, pp. 278-282, 2002.

[153] Wallin, M., Curtin, W. A., RistinmaA, M., and Needleman, A., "Multi-scale plasticity modeling: Coupled discrete dislocation and continuum crystal plasticity," Journal of the Mechanics and Physics of Solids, vol. 56, no. 11, pp. 3167-3180, 2008.

[154] Wen, M., Takahashi, A., and Ghoniem, N. M., "Kinetics of self-interstitial cluster aggregation near dislocations and their influence on hardening," Journal of Nuclear Materials, vol. 392, pp. 386-395, Aug 2009.

[155] Wilson, W. in: Conference on Fundamental Aspects of Radiation Damage in Metals, vol. USERDA-CONF-751006-P2, p. 1025, 1975.

[156] Wilson, W., Bisson, C., and Baskes, M., "Self-trapping of helium in metals," Physical Review B, vol. 24, no. 10, pp. 5616-5624, 1981.

[157] Woodward, C. and RAO, S., "Ab-initio simulation of isolated screw dislocations in bcc Mo and Ta," Philosophical Magazine A, vol. 81, no. 5, pp. 1305-1316, 2001.

[158] Zвib, H. and Aifantis, E., "Size effects and length scales in gradient plasticity and dislocation dynamics," Scripta Materialia, vol. 48, no. 2, pp. 155-160, 2003. 
APPENDIX 2

MODELING THE MECHANICAL BEHAVIOR AND DEFORMED MICROSTRUCTURE OF IRRADIATED BCC MATERIALS USING CONTINUUM CRYSTAL PLASTICITY 


\section{SUMMARY}

Appendix 2: Modeling the mechanical behavior and deformed microstructure of irradiated bcc materials using continuum crystal plasticity contains content from 4 refereed publications

The mechanical behavior of structural materials used in nuclear applications is significantly degraded as a result of irradiation, typically characterized by an increase in yield stress, localization of inelastic deformation along narrow dislocation channels, and considerably reduced strains to failure. Further, creep rates are accelerated under irradiation. These changes in mechanical properties can be traced back to the irradiated microstructure which shows the formation of a large number of material defects, e.g., point defect clusters, dislocation loops, and complex dislocation networks. Interaction of dislocations with the irradiation-induced defects governs the mechanical behavior of irradiated metals. However, the mechanical properties are seldom systematically correlated to the underlying irradiated microstructure. Further, the current state of modeling of deformation behavior is mostly phenomenological and typically does not incorporate the effects of microstructure or defect densities.

The present research develops a continuum constitutive crystal plasticity framework to model the mechanical behavior and deformed microstructure of bcc ferritic/martensitic steels exposed to irradiation. Physically-based constitutive models for various plasticity-induced dislocation migration processes such as climb and cross-slip are developed. We have also developed models for the interaction of dislocations with the irradiation-induced defects. A rate theory based approach is used to model the evolution of point defects generated due to irradiation, and coupled to the mechanical behavior. A void nucleation and growth based damage framework is also developed to model failure initiation in these irradiated materials. The framework is used to simulate the following major features of inelastic deformation in bcc ferritic/martensitic steels: irradiation hardening, flow localization due to dislocation channel formation, failure initiation at the interfaces of these dislocation channels and grain boundaries, irradiation creep deformation, and temperature-dependent non-Schmid yield behavior. Model predictions are compared to available experimental data.

This framework represents the state-of-the-art in constitutive modeling of the deformation behavior of irradiated materials. 


\section{CHAPTER 1}

\section{INTRODUCTION}

\subsection{Motivation}

In recent years, there has been a renewed interest in nuclear energy as a sustainable and relatively clean source for our present and future energy requirements. Design of nuclear power generation systems is contingent on the development of structural alloys that can withstand extreme stress and temperature loading in the presence of radiation fluxes and corrosive environments over the life of these power generation systems. In particular, the presence of irradiation poses a key challenge in extending the life of these structural materials, which are used in the cladding and core structures of nuclear pressure vessel reactors. Varied and complex radiation-induced phenomena occur over multiple length- and time-scales that degrade the mechanical properties of these materials, and their study is a matter of ongoing research. While significant efforts have been directed towards studying the effects of radiation on microstructure evolution, these microstructural features are seldom systematically correlated to the deformation behavior and mechanical properties at the meso- and macro-scale, especially in terms of constitutive modeling.

Understanding the effect of radiation-induced defects on the dislocation-mediated crystallographic deformation behavior holds the key for modeling and designing 'radiationresistant' structural alloys for nuclear applications. The focus of the present research is directed at understanding these dislocation-defect interaction mechanisms and developing physicallybased continuum constitutive equations at the level of crystal plasticity to model the mechanical behavior and deformed microstructure of irradiated alloys.

\subsection{Radiation damage in metallic systems}

Radiation damage in metallic crystals is initiated by the 'knock-on' event where an incident energetic particle interacts with a lattice atom and transfers a large part of its kinetic energy to the lattice atom. This transfer of energy results in the creation of the primary knock-on atom (PKA) as the 'excited' lattice atom leaves its lattice site and traverses through the lattice creating more PKAs and accompanying defects. When the PKAs have expended all their energy, they finally terminate as interstitials in the lattice. The physics of radiation damage and defect production has been explained in detail by Was [6].

Material defects created during the radiation damage process include point defect clusters, dislocation loops and networks, voids, bubbles, precipitates, solute segregation at grain boundaries, etc. Interaction of the plasticity-carrying dislocations with these defects alters their 
deformation behavior and mechanical properties. Primary observed effects include irradiation hardening, plastic flow localization, reduced ductility and significantly lower strains to failure. Further, the irradiation creep rates are accelerated as compared to thermal creep, and occur at lower homologous temperatures [7]. These effects are generally observed in most metallic systems and are a key concern for the use of structural materials in reactor vessels.

The operating conditions and mechanical properties of nuclear structural materials are ever-evolving and there are various classes of materials depending on their application. The present generation $9-12 \% \mathrm{Cr}$ ferritic/martensitic steels used as cladding material are expected to operate in the temperature range $300-600^{\circ} \mathrm{C}$ and can take radiation doses up to $70-80 \mathrm{dpa}$ (radiation damage measured in average displacements per atom) [8]. These bcc ferritic/martensitic steels have higher swelling resistance and better creep properties than the previous generation fcc austenitic steels [9], and are candidates for fusion and Generation IV fission applications [10]. A summary of the tensile strength and fracture properties of various ferritic/martensitic steels is given by Anderoglu et al. [11]. The present work focuses on modeling the mechanical properties of these bcc ferritic/martensitic steels. While the constitutive equations developed may be applied to bcc materials in general, we have used Mod 9Cr-1Mo ferritic/martensitic steel as a model material in most of this work.

\subsection{Scope of the present research}

This research develops a continuum constitutive crystal viscoplasticity framework to model radiation damage in bcc materials over a wide range of deformation and loading histories. While crystal plasticity frameworks for modeling radiation damage have been developed previously [12-15], they generally address only the initial irradiation hardening and the posthardening flow localization during tensile loading. In the present work, a unified model framework is developed to address: both irradiation hardening and flow localization during postirradiation quasi-static tensile loading; failure initiation subsequent to plastic flow localization during tensile loading; and in-situ irradiation creep deformation. While separate deformation mechanisms may be dominant in each of these deformation regimes, physically, none of them are expected be mutually exclusive. This allows us to develop a unified model framework with different deformation mechanisms dominant during different loading regimes. Example applications of the framework to model each of the above phenomena are given separately.

In this crystal plasticity framework, defect densities representative of the substructure (mobile dislocations, immobile dislocations, and irradiation-induced defects), rather than phenomenological hardening variables, are used as internal state variables. Physically-based constitutive equations are developed to model the interactions among these defect variables. Many of these constitutive equations draw on information obtained from lower length- and timescale simulations (e.g., molecular dynamics, and kinetic Monte Carlo) of the interaction of 
dislocations with irradiation-induced defects. A major emphasis of this research lies on modeling not just the mechanical properties, but the deformed microstructure as well. This allows us to correlate the observed mechanical behavior to the underlying spatial and temporal substructure evolution. Model results and predictions are compared to available experimental data.

\subsection{Organization of this report}

The modeling tasks described above are organized in this report in the following fashion.

Chapter 2 starts with a discussion of the microstructural effects of irradiation and the observed deformation mechanisms. A brief review of the prior constitutive modeling efforts is also given. We then discuss the development of a crystal plasticity framework to model irradiation damage. We start with a description of the internal state variables used in this framework, followed by the finite deformation kinematics of the crystal plasticity framework. Constitutive equations for the thermally-activated dislocation glide kinetics of double kink nucleation (in bcc metals) and point defect flux-governed dislocation climb are then discussed in the context of a conservative crystal plasticity framework. Rate equations for irradiation-induced point defects and interstitial loops are then developed and coupled with the deformation phenomena. Constitutive equations for dislocation density evolution are also developed. Climb and cross-slip of dislocations is modeled and tied with the deformation kinetics. The framework is used to model irradiation hardening in a Mod $9 \mathrm{Cr}-1 \mathrm{Mo}$ ferritic/martensitic steel during tensile loading. Model predictions are also made for irradiation creep loading at different applied stresses and radiation rates.

Chapter 3 focuses on modeling flow localization in irradiated bcc materials due to dislocation channel formation. Current understanding of the dislocation channeling phenomena in bcc metals is discussed. A phenomenological constitutive equation is developed to model flow localization due to the clearing of irradiation-induced defects along preferred crystallographic planes, allowing subsequent dislocation glide to occur. This constitutive equation is coupled with the existing framework to model the mechanical behavior and deformed microstructure due to dislocation channel formation. Effect of various mesoscopic factors such as initial crystallographic orientation distribution, grain size, radiation dose, and degree of cross-slip on the localization behavior is also studied.

Chapter 4 develops a continuum damage framework to model failure initiation at the interfaces of dislocation channels, subsequent to flow localization. Constitutive equations for the generation of vacancies during inelastic deformation are developed and coupled to the nucleation and growth of voids by vacancy-mediated processes. The damage framework is coupled with the existing crystal plasticity framework to model failure initiation ahead of a sharp notch. The numerical simulations are used to measure the crack-tip displacements and compute the crack 
growth resistance. Fracture toughness calculations are also performed for the irradiated specimens.

Chapter 5 models the mechanical behavior and microstructure evolution during irradiation creep deformation. A constitutive model for the absorption of irradiation-induced point defects at grain boundary sinks is developed and coupled with the existing crystal plasticity framework. The creep strain is correlated with the evolution of radiation damage (measured in dpa). The deformed microstructure is studied in terms of the spatial distribution of dislocations and irradiation-induced defects. Effect of varying the grain boundary density on the creep strain rates is also studied.

Chapter 6 develops constitutive equations for modeling non-Schmid yield effects due to dislocation core spreading in bcc metals. Constitutive equations to model the temperature dependence of non-Schmid coefficients are developed based on available experimental data for bcc-iron. Decay of non-Schmid effects with increasing inelastic deformation is also considered in this model. The constitutive equations are used to model the orientation-dependent mechanical behavior of single crystal iron over different temperatures. Model predictions for tensioncompression asymmetry in bcc-iron are also made. Implications for irradiation damage and effect of the presence of interstitials near the screw dislocation core on the non-Schmid yield behavior are also discussed.

Finally, Chapter 7 summarizes the novel contributions and major conclusions of this research, along with recommendations for future work.

Note that Chapters 3 through 6 develop models for addressing different aspects of the deformation behavior of irradiated bcc metals. If interested in one specific aspect of the deformation behavior, the reader may refer to that particular Chapter, after an understanding of the constitutive model framework described in Chapter 2. Further, a separate Chapter on literature review has been foregone, since each Chapter has a rather comprehensive literature review pertaining to the topics discussed in that Chapter. 


\section{CHAPTER 2}

\section{A CONSTITUTIVE CRYSTAL PLASTICITY FRAMEWORK FOR MODELING RADIATION DAMAGE IN BCC MATERIALS ${ }^{1}$}

\subsection{Introduction}

The quasi-static tensile behavior of metals subject to radiation damage is typically characterized by an increase in the yield point and decrease of uniform ductility and true strain to fracture. As for creep behavior, the 'irradiation creep' rates have been found to be much higher than the thermal creep rates in virgin materials and occur at relatively lower temperatures. Radiation damage reduces the life of these structural materials used in nuclear applications. Accordingly, research is directed at understanding the mechanisms governing inelastic deformation and improving material performance.

Inelastic deformation of irradiated materials at ambient and intermediate temperatures is essentially a multiscale phenomenon governed by unit processes spanning length scales over more than 15 orders of magnitude and time scales spanning 21 orders of magnitude [17]. Starting with localized shuffling of the lattice due to incident, high-energy, knock-on atoms and radiation cascade-induced defect nucleation and growth [18], and subsequent spatial evolution (migration and clustering) of these point defects [19] and their interaction with dislocations [20,21], this leads to irradiation-induced hardening, and eventually dislocation channeling and flow localization [22]. A multitude of physical phenomena occur in cascade from the atomic lengthand timescales to the length- and timescales of engineering structures.

For modeling the mechanical behavior of such bcc ferritic/martensitic structural materials at the scale of microstructure (e.g., individual grains), it is necessary to consider crystallographic deformation mechanisms based on dislocation-defect interactions and their evolution. Continuum constitutive models should rely on information from lower length- and time-scale models of reaction pathways for dislocation nucleation and barrier bypass, and more importantly the point defect-dislocation reaction kinetics for irradiated materials. This Chapter will discuss a mechanism-based internal state variable (ISV) approach coupled with continuum crystal plasticity to model inelastic deformation in bcc materials subjected to radiation damage at ambient and intermediate homologous temperatures under quasi-static and creep loading.

\footnotetext{
${ }^{1}$ Significant parts of this Chapter have appeared in Ref. [16].
} 


\subsection{Background}

\subsubsection{Microstructural effects of radiation}

Irradiation of metals produces a large number of defects (interstitials and vacancies) that affect their mechanical properties. Depending on the type of metal (fcc or bcc), certain distinct microstructural effects can be observed. For fcc metals such as $\mathrm{Cu}$, stacking fault tetrahedra form the majority of the irradiation-induced defects [23]. For bcc metals such as Fe, interstitial loops are the primary observed defect structures [24]. Complex dislocation networks, cavities and precipitates are also observed [25]. Another common feature of irradiated deformed microstructures is the formation of defect-free channels and corresponding plastic flow localization $[22,26]$.

Dislocation networks act as sinks for point defects. Since a high density of dislocations is generated, the effective dislocation sink strength for these defects in irradiated materials is higher than the grain boundary sink strength. Swelling is another phenomenon observed due to the formation of voids in the lattice [27]. Voids provide a neutral sink facilitating diffusion of excess interstitials to the dislocations. Also observed is the biased absorption of interstitials at dislocations, promoting dislocation climb at barriers $[28,29]$. The focus of the present work is modeling the effect of these defects on the mechanical behavior of the material during and postirradiation.

At intermediate to elevated homologous temperatures (>0.3), inelastic deformation becomes strain-rate and temperature sensitive (thermally activated dislocation migration). In addition to glide, dislocation climb also contributes to inelastic deformation at these temperatures [30], particularly in the presence of irradiation-induced defects. This is affected by the nonequilibrium concentration of point defects, allowing dislocations to climb by absorption or emission of point defects. This is commonly referred to as "climb-controlled" creep [30,31].

For irradiated materials, the creep rate is considerably higher than that in thermal creep (of virgin materials) and occurs at much lower temperatures [6]. Deformation mechanism maps show that irradiation creep occurs at homologous temperatures of the order 0.3-0.5 as compared to thermal creep which occurs at homologous temperatures greater than 0.5 [7]. As opposed to thermal creep, which is governed by diffusion-controlled processes, accelerated irradiation creep rate is attributed to the creation of a large number of point defects that promote the growth and migration of defect loops and voids by stress-assisted mechanisms.

\subsubsection{Irradiation creep mechanisms}

A number of irradiation creep mechanisms have been conjectured, none of which by themselves have been able to explain the deformation behavior of materials over the entire 
domain of radiation damage. A comprehensive review of the various irradiation creep mechanisms is given by Bullough and Wood [32] and Matthews and Finnis [33].

Brailsford and Bullough [34] suggested that upon the application of external uniaxial stress, interstitial loops are more likely to nucleate on crystallographic planes with preferred orientation, specifically on planes perpendicular to the applied stress; while vacancy loops nucleate parallel to the direction of applied stress. This mechanism (stress induced preferential nucleation, SIPN) ultimately results in elongation of the material along the direction of applied stress. It can be shown that the creep rate in this case is linearly proportional to the applied stress. This model for creep, though supported by some experimental evidence, does not account for all the observed creep behaviors [6]. However, SIPN can be used to account for creep during the transient creep regime when interstitial loops start nucleating.

The stress-induced preferential absorption (SIPA) model for steady-state irradiation creep was proposed originally by Heald and Speight [35] and later quantitatively analyzed by Bullough and Willis [36]. Under the application of external stress, interstitials drift preferentially towards planes perpendicular to the direction of applied stress. The physical basis of this model is that the stress-induced interaction energy between preferentially aligned edge dislocations and interstitials is higher than that between non-aligned dislocations and interstitials for an applied tensile stress. This preferential drift of interstitials facilitates climb of edge dislocations perpendicular to the plane by absorption of interstitials. The climb contribution to creep strain has been shown to be a linear function of the applied stress and is in reasonable agreement with steady-state creep data for steels [37].

The SIPA mechanism accounts only for climb of edge dislocations by absorption of interstitials under applied external stress. Mansur and Reiley [28,38] suggested that there must be an additional component of creep strain due to the subsequent glide of dislocations on the crystallographic plane, after the edge component of dislocations pinned at interstitials have climbed by SIPA. This climb-enabled glide of dislocations, also known as preferred absorption glide (PAG) has been shown to provide a dominant contribution to the creep strain at lower stresses and low dislocation densities [38].

\subsubsection{Summary of prior constitutive modeling efforts}

A multitude of phenomenological and physics-based constitutive models have been developed in the past to model plasticity and creep in irradiated materials. A brief review of some of the models is given here.

Ghoniem et al. [39] developed a model for creep (GMA model) based on unit dislocation evolution mechanisms. This model can be integrated with rate theory-based evolution of point defects in irradiated materials. This model has evolution laws for dislocation growth and 
migration processes, based on the interaction of dislocations with other (point) defects. The GMA model laid a comprehensive framework for linking physical dislocation mechanisms of deformation to the mechanical behavior of materials at high temperatures.

The mechanical behavior of irradiated materials characterized by an initial increase in yield stress has been correlated to the observed microstructural changes and modeled using a dispersed barrier hardening $(\mathrm{DBH})$ model $[40,41]$. This model tries to establish a direct relation between the change in yield strength and the evolution of defect densities. Singh et al. [42] developed a cascade-induced source hardening (CISH) model wherein the increase in yield strength is interpreted as the added stress necessary to free the grown-in dislocations from irradiation-induced defect loops, thus enabling them to act as Frank-Read sources for dislocation multiplication and initiating plastic deformation.

Arsenlis et al. [12] discussed a continuum dislocation density-based plasticity model for irradiated fcc metals at low homologous temperatures. In this model, the material strength is derived from impediments to dislocation glide in the form of forest dislocations and irradiationinduced defects (stacking fault tetrahedra). The dislocation densities in this model evolve by dislocation multiplication and pair-wise annihilation mechanisms and the defects evolve by shearing of the initially perfect defects by passing dislocations.

Krishna et al. [13] developed a micromechanics-based rate-independent crystal plasticity model to capture the quasi-static tensile behavior of OFHC copper subject to radiation damage. The critical resolved shear stress on each slip system has a square root dependence on the dislocation and defect densities in this model. An evolution law for irradiation-induced defects based on experimental observation of annihilation of these defects, when they are within a prescribed standoff distance of the dislocations [43], was also proposed in this work.

Li et al. [14] developed a multiscale model for predicting irradiation hardening in ironbased alloys. Dislocation dynamics (DD) simulations of interactions between dislocations and irradiation-induced defects were used to compute the average critically resolved shear stress (CRSS) as a function of the defect density in irradiated iron, which was then fed into a phenomenological viscoplastic crystal plasticity framework to model the irradiation hardening and texture evolution. Simulation results were then compared with experiments to establish the hardening behavior as a function of radiation damage (in dpa).

More recently, Barton et al. [15] developed a crystal plasticity framework for modeling strain localization in irradiated iron. The slip system strength in this model evolves as a function of the network dislocation density and a tensor damage variable representative of the 'contact density' between dislocations and irradiation-induced prismatic loops. The crystal plasticity model was calibrated using stress-strain curves of irradiated iron with varying loop densities, obtained from DD simulations. The DD simulations performed by Arsenlis et al. [44] show that elastic interactions of the prismatic loops with gliding dislocations leads to their coarsening and 
coalescence, until the coarsened loops resemble network dislocations (in virgin, unirradiated materials) in their hardening behavior, thus leading to lower threshold stress for glide within the channels. To account for this flow softening mechanism, Barton et al. [15] developed a phenomenological rule assuming exponential decay of the prismatic loop density.

Most of the models developed to date do not provide a complete description of the underlying dislocation-defect interaction processes at the level of crystal plasticity in irradiated materials. Further, mechanical behavior of bcc metals is governed by more complex crystallographic deformation mechanisms such as cross-slip of screw dislocations and nonSchmid yield behavior due to dislocation core spreading. To account for all of the above mentioned physical phenomena, a mechanism-based crystal plasticity model will be developed for irradiated bcc systems in this work. In the following sections, the continuum constitutive model will be explained and then the model will be exercised to qualitatively predict the quasistatic tensile and creep behavior of a bcc ferritic/martensitic steel as a function of irradiation exposure.

\subsection{Model description}

The following sections will explain the Internal State Variables (ISVs) adopted in this model, the finite deformation kinematics of crystal plasticity, constitutive formulation of inelastic deformation and hardening laws, and the evolution laws of the ISVs.

\subsubsection{Defect densities as internal state variables}

This work focuses on bcc ferritic/martensitic steels, where interstitial loops are the primary irradiation-induced defects and vacancy loops are not observed (even with transmission electron microscopy) [24]. Interstitial loops are formed in irradiated materials when interstitialclusters (formed during the displacement cascade) condense onto close-packed planes in the lattice.

Number densities of the interstitial loops, $N_{i}^{\alpha}$, and their mean size, $d_{i}^{\alpha}$, are therefore used as ISVs in this model. They evolve with cumulative irradiation damage, measured in terms of displacements per atom (dpa). It is assumed that all the interstitial loops are sessile. Furthermore, the dislocation density, $\rho^{\alpha}$, on each slip system $\alpha$, comprised of dislocations other than interstitial loops, is also tracked. Along the lines of the work of Estrin and Kubin [45], the dislocation density is additively decomposed into mobile and immobile parts to help capture the transients in plastic flow, i.e., $\rho^{\alpha}=\rho_{M}^{\alpha}+\rho_{I}^{\alpha}$, where $\rho_{M}^{\alpha}$ is the mobile dislocation density and $\rho_{I}^{\alpha}$ is the immobile dislocation density on slip system $\alpha$. Apart from the planar and line defects, this model also tracks the concentration of irradiation-induced point defects (interstitials, $c_{i}$, and 
vacancies, $c_{v}$ ) that play an important role in dislocation migration processes. These point defects also evolve with dpa. The evolution of these ISVs is discussed in Section 2.3.3.3.

\subsubsection{Finite deformation kinematics}

The constitutive crystal plasticity formulation is based on multiplicative decomposition of the deformation gradient tensor, $\boldsymbol{F}$, into the elastic, $\boldsymbol{F}^{e}$, and inelastic, $\boldsymbol{F}^{i}$, parts [46,47], i.e.,

$$
\boldsymbol{F}=\boldsymbol{F}^{e} \cdot \boldsymbol{F}^{i}
$$

with $\boldsymbol{F}^{i}$ describing the effects of the inelastic deformation on an undeformed lattice, mapping the reference configuration to an intermediate configuration in which the lattice has the same orientation, and $\boldsymbol{F}^{e}$ describes the lattice distortion and the rigid body rotation that carries the intermediate isoclinic configuration to the current, deformed configuration. Moreover, $\dot{\boldsymbol{F}}^{i}=\boldsymbol{L}^{i} \cdot \boldsymbol{F}^{i}$, where $\boldsymbol{L}^{i}$ is the inelastic velocity gradient determined by the crystallographic shearing rates on various slip systems, i.e.,

$$
\boldsymbol{L}^{i}=\sum_{\alpha=1}^{N_{s}} \dot{\gamma}^{\alpha} \boldsymbol{m}_{0}^{\alpha} \otimes \boldsymbol{n}_{0}^{\alpha}
$$

where $\boldsymbol{m}_{0}^{\alpha}$ and $\boldsymbol{n}_{0}^{\alpha}$ are unit vectors corresponding to the slip and slip plane normal directions in the reference (or intermediate) configurations, $\dot{\gamma}^{\alpha}$ is the shearing rate on slip system $\alpha$, and $N_{s}$ is the total number of slip systems.

\subsubsection{Constitutive model}

\subsubsection{Elastic deformation}

For infinitesimal lattice deformation, the elastic Green strain tensor, $\boldsymbol{E}^{e}=(1 / 2)\left(\boldsymbol{F}^{e T} \cdot \boldsymbol{F}^{e}-\boldsymbol{I}\right)$, is related to the second Piola-Kirchhoff (PK) stress tensor in the intermediate configuration by $\boldsymbol{S}=\boldsymbol{C}: \boldsymbol{E}^{e}$, where $\boldsymbol{C}$ is the fourth rank elasticity tensor of the lattice. The PK stress tensor, $S$, is related to the Cauchy stress, $\sigma$, in the current configuration by $\boldsymbol{S}=\operatorname{det}\left(\boldsymbol{F}^{e}\right) \boldsymbol{F}^{e-1} \cdot \boldsymbol{\sigma} \cdot \boldsymbol{F}^{e-T}$. The resolved shear stress on a slip plane is given by Schmid's law, i.e.,

$$
\tau^{\alpha}=\boldsymbol{m}^{\alpha} \cdot \boldsymbol{\sigma} \cdot \boldsymbol{n}^{\alpha}=\frac{1}{\operatorname{det}\left(\boldsymbol{F}^{e}\right)} \boldsymbol{m}_{0}^{\alpha} \cdot \boldsymbol{S} \cdot \boldsymbol{n}_{0}^{\alpha}
$$


where $\boldsymbol{m}^{\alpha}$ and $\boldsymbol{n}^{\alpha}$ are the unit vectors corresponding to the slip and slip plane normal direction in the current configuration.

\subsubsection{Inelastic deformation}

Inelastic deformation in crystalline materials is governed by dislocation migration on close-packed slip planes with preferred orientations. This is represented at the continuum level in terms of the crystallographic shearing rate for each slip system. The physical picture of inelastic deformation can be visualized as follows: mobile dislocations glide on preferred slip planes and get pinned at barriers, which in this case are primarily in the form of interstitial loops and immobile dislocations. Parts of the pinned mobile dislocation segments then climb by absorbing point defect clusters, allowing the unpinned dislocation segments to glide further. There are therefore two contributions to the crystallographic shearing rate, one due to thermally-activated glide of dislocation segments and the other due to climb of the edge component of dislocations, assisted by preferred absorption of interstitials.

\subsection{Dislocation glide}

Dislocation glide in bcc metals is governed by the thermally-activated double kink nucleation mechanism. The local, short-range barriers to dislocation glide (e.g., Peierls barrier) can be overcome by thermal fluctuations, while the long-range barriers with higher activation energy for bypass, due to the presence of immobile dislocations and interstitial loops (in this case), cannot be readily overcome by thermal fluctuations. Kothari and Anand [48] formulated a law for the dislocation glide velocity using a Kocks-type activation enthalpy term [49], i.e.,

$$
v_{g}^{\alpha}=\left\{\begin{array}{l}
l_{g} v \exp \left(-\frac{\Delta F_{g}}{k T}\left(1-\left(\frac{\left|\tau^{\alpha}\right|-s_{a}^{\alpha}}{s_{t}^{\alpha}}\right)^{p}\right)^{q}\right) \operatorname{sgn}\left(\tau^{\alpha}\right) ; \text { for }\left|\tau^{\alpha}\right|>s_{a}^{\alpha} \\
0 ; \quad \text { for }\left|\tau^{\alpha}\right| \leq s_{a}^{\alpha}
\end{array}\right.
$$

where $l_{g}$ is the glide distance between barriers, $v$ is the cooperative attempt frequency of dislocation glide, $\Delta F_{g}$ is the activation energy barrier in the absence of external applied stress, $k$ is the Boltzmann constant, $T$ is the absolute temperature, $s_{a}^{\alpha}$ is the athermal barrier resistance, $s_{t}^{\alpha}$ is the thermal barrier resistance, and $p$ and $q$ are parameters used to model the shape of the activation enthalpy curve.

Based on a dispersed barrier hardening model [40,41,50], the athermal slip resistance due to long-range interactions of dislocations with interstitial loops and dislocations on other slip systems depends on the dislocation densities, defect loop sizes and corresponding number densities, is given by 


$$
s_{a}^{\alpha}=G b \sqrt{\sum_{\zeta=1}^{N_{s}} q_{\rho} A^{\alpha \zeta} \rho^{\zeta}+q_{i} N_{i}^{\alpha} d_{i}^{\alpha}}
$$

where $q_{\rho}$ is the dislocation barrier strength, $q_{i}$ is the interstitial loop strength, and $A^{\alpha \zeta}$ is the matrix of slip system dislocation interaction coefficients. We neglect here any long-range directional contribution to $s_{a}^{\alpha}$ (e.g., slip system back stress), as an approximation in view of the propensity for SIPA and screw dislocation cross-slip. It is noted that Bauschinger effects can still arise due to the spatial heterogeneity of yielding associated, for example, with the orientation distribution of grains (cf. [51]). Further, the effect of non-Schmid stresses in constricting the screw dislocation core in bcc materials [52-57] has also been neglected in the constitutive formulation and examples in this Chapter. It is expected that increase in interstitial concentration in irradiated materials will further hinder the constriction of dislocation cores, leading to a more pronounced effect of core spreading. Models to address these non-Schmid stresses are discussed in Chapter 6.

The thermal slip resistance, $s_{t}^{\alpha}$, for bcc materials is typically governed by the (high) Peierls lattice resistance, $\tau_{p}$, i.e., $s_{t}^{\alpha}=\tau_{p}$.

\subsection{Dislocation climb}

Climb in irradiated materials is driven by interaction of edge dislocation segments with the local non-equilibrium concentration of point defects (interstitials and vacancies). Climb can occur either by interstitial or vacancy trapping at the edge dislocation core. The climb rate is thus governed by the flux of interstitials and vacancies near the core of the edge dislocation segment. The dependence of dislocation climb velocity on the flux of point defects to and from the core is given as [38]

$$
v_{c}^{\alpha}=\frac{\Omega}{b}\left(z_{i}^{\alpha} D_{i} c_{i}-z_{v}^{\alpha} D_{v} c_{v}\right)
$$

where $\Omega$ is the atomic volume, $z_{i}^{\alpha}, z_{v}^{\alpha}$ are the interstitial and vacancy capture efficiencies (defined as fraction of the total available point defects absorbed) of the dislocations perpendicular to the slip plane, respectively, and $D_{i}, D_{v}$ are the effective interstitial and vacancy diffusivities, respectively. Here, $c_{i}, c_{v}$ are the previously mentioned bulk interstitial and vacancy concentrations, respectively. Physically, the capture efficiencies, $z_{i}^{\alpha}$ and $z_{v}^{\alpha}$, are affected by the atomic sites around a dislocation core available for the respective point defect absorption. The 
first term in the above expression represents the climb rate due to interstitial trapping and the second term represents the drag due to vacancy trapping at the dislocation core.

It has been further suggested that the interstitial capture efficiency, $z_{i} \propto \sigma / E$, where $\sigma$ is the uniaxial stress and $E$ is the Young's modulus [38]. Extending this to a crystal plasticity framework, we use $z_{i}^{\alpha}=z_{i}^{0}\left(\sigma_{c}^{\alpha} / E\right)$, where $\sigma_{c}^{\alpha}=\boldsymbol{n}^{\alpha} \cdot \boldsymbol{\sigma} \cdot \boldsymbol{n}^{\alpha}$ is the resolved stress normal to the slip plane; $\sigma_{c}^{\alpha}$ corresponds to the climb component of the Peach-Koehler force on a dislocation [58]. The coefficient, $z_{i}^{0}$, in the above expression is assumed to be constant over all stress and temperature ranges. This expression reflects the dependence of interstitial capture efficiency on stress, thus differentiating between dislocations that are aligned and those that are not aligned to the stress direction (SIPA). Note that this expression also gives the direction in which dislocation climb occurs, namely $\boldsymbol{n}^{\alpha}$. A similar expression follows for vacancy capture efficiency, i.e., $z_{v}^{\alpha}=z_{v}^{0}\left(\sigma_{c}^{\alpha} / E\right)$.

The bias for absorption of interstitials as compared to vacancies (due to the preferential mutual interaction fields of interstitials with dislocations) will be accounted for in the coefficient, $z_{i}^{0}$, having a higher value than $z_{v}^{0}$. For simplicity, interaction of point defects with either edge or screw type of dislocations has not been explicitly differentiated in the present formulation. Note that all of the interactions mentioned in this section are those with edge dislocations. As mentioned earlier, interactions of point defects with screw dislocations will lead to a more pronounced dislocation core spreading effect. Future work will focus on modeling these interactions based on input from atomistics.

\subsection{Crystallographic shearing rate due to climb and glide}

Since dislocation climb occurs perpendicular to the slip plane, it does not contribute to inelastic strain in the same manner as dislocation glide. Further, climb by itself is a nonconservative process; hence modeling this in a crystal plasticity framework is not straightforward. Based on the physical picture of inelastic deformation presented earlier, glide on the slip plane is assisted by climb events which enable dislocations to overcome the barriers to glide in the domain of lower (insufficient) stresses. Climb is accompanied by migration of mobile dislocations via absorption of point defects. A schematic of this mechanism is shown in Fig. 1. Hence, there is a contribution of climb to the crystallographic shearing rate, in addition to the glide contribution of mobile dislocations. The inelastic shear strain on a slip plane due to glide of mobile dislocations is given by the Orowan equation [59], i.e.,

$$
\gamma^{\alpha}=\rho_{M}^{\alpha} b l_{g}
$$


where $l_{g}$ is the average distance swept by glide dislocations on the slip plane. Taking derivative of this equation with respect to time leads to the expression for inelastic shearing rate, i.e., $\dot{\gamma}^{\alpha}=\rho_{M}^{\alpha} b v_{g}^{\alpha}+\dot{\rho}_{M}^{\alpha} b l_{g}$. The first term of this expression represents the shearing rate due glide, i.e., $\dot{\gamma}_{g}^{\alpha}=\rho_{M}^{\alpha} b v_{g}^{\alpha}$, and can be calculated from Eq. (2.4), if $\rho_{M}^{\alpha}$ is known. The second term is assumed here to represent the contribution to the crystallographic shearing rate due to climb, $\dot{\gamma}_{c}$, and is generally negligible in the quasi-static regime at lower homologous temperatures or for virgin (unirradiated) materials at moderate to high levels of mobile dislocation density. However, it is significant in the SIPA regime, when dislocation glide becomes increasingly difficult due to the presence of sessile interstitial loops which dominate the landscape of obstacles, and elevated temperatures result in higher diffusivities of point defects.

The frequency of dislocation climb events reckoned in terms of the population of mobile dislocations is given by $f_{c}^{\alpha}=v_{c}^{\alpha} / l_{c}$, where $v_{c}^{\alpha}$ is the mean climb velocity of mobile edge segments and $l_{c}$ is the average distance climbed. The corresponding rate of migration of mobile dislocations passing barriers due to climb is therefore given by $f_{c}^{\alpha} \rho_{M}^{\alpha}$. Accordingly, we can write the contribution to the crystallographic strain rate due to climb as a function of the migration rate of mobile dislocations, i.e.,

$$
\dot{\gamma}_{c}^{\alpha}=f_{c}^{\alpha} \rho_{M}^{\alpha} b l_{g}=\frac{v_{c}^{\alpha}}{l_{c}} \rho_{M}^{\alpha} b l_{g}
$$

The total crystallographic shearing rate on slip system $\alpha$ is therefore given as $\dot{\gamma}^{\alpha}=\dot{\gamma}_{g}^{\alpha}+\dot{\gamma}_{c}^{\alpha}=\rho_{M}^{\alpha} b v_{g}^{\alpha}+\dot{\gamma}_{c}^{\alpha}$.

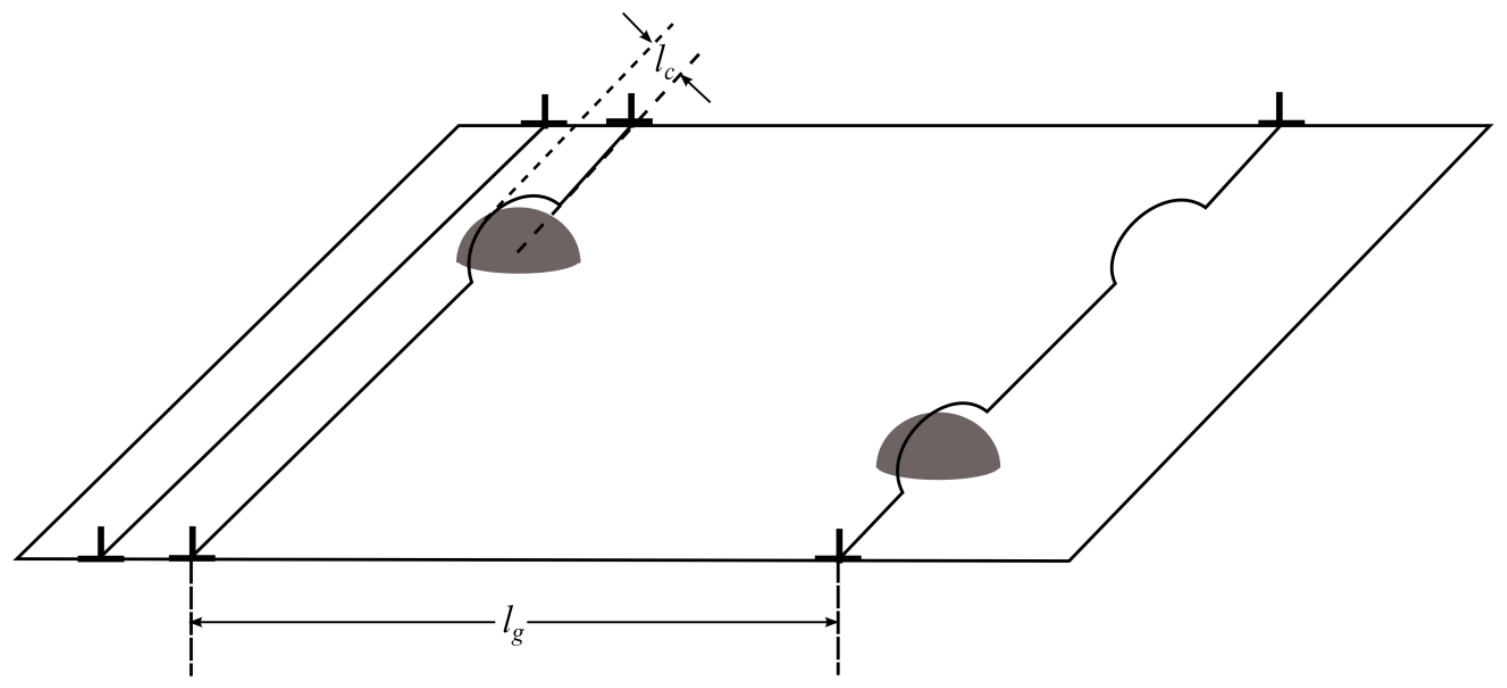

Figure 1. A dislocation on a slip plane climbs across a barrier of height, $l_{c}$, and is free to glide to the next obstacle; $l_{g}$ is the slip distance after which another such barrier is encountered. 


\subsubsection{Substructure evolution}

This section deals with the evolution of point, line and planar defects, which constitute the substructure of the material. The evolution equations for all defects are in the form of rate equations, with individual terms representing specific physical mechanisms of defect generation, depletion, and recovery. First we will consider point defects, and then move on to interstitial loops, followed by dislocations. The models presented here offer a continuum scale statistical representation of the mechanisms governing evolution of these defects as a collective ensemble, and is limited in applicability to perhaps the lesser of 10 times the mean dislocation spacing or $10 l_{g}$ in minimum scale.

\subsection{Point defects}

Point defects are generated due to continuous, sequential collisions of incident highenergy radiation-induced particles with lattice atoms during radiation, knocking atoms off their respective lattice sites and creating interstitial-vacancy pairs. A part of the interstitial and vacancy population recombines over time to regenerate ordered lattice arrangement. Some point defects are subsequently absorbed by the mobile dislocations to form interstitial loops, while others are absorbed by network (immobile) dislocations. Radiation-induced point defects thus evolve by the following competing processes: defect pair creation by collisions between incident high-energy particles and lattice atoms, defect pair recombination, and defect absorption by dislocations. It is assumed for simplicity that the point defects are homogeneously distributed within the material and there are no spatial concentration gradients. The corresponding local rate equations for interstitial and vacancy concentrations are thus given as

$$
\begin{aligned}
& \dot{c}_{i}=K_{0}-K_{i v} c_{i} c_{v}-\sum_{\alpha} K_{i s}^{\alpha}\left(\rho_{M}^{\alpha}+\rho_{I}^{\alpha}\right) c_{i} \\
& \dot{c}_{v}=K_{0}-K_{i v} c_{i} c_{v}-\sum_{\alpha} K_{v s}^{\alpha}\left(\rho_{M}^{\alpha}+\rho_{I}^{\alpha}\right) c_{v}
\end{aligned}
$$

where $K_{0}, K_{i v}$ are the defect pair creation and recombination rates, respectively, $K_{i s}^{\alpha}=\left|z_{i}^{\alpha}\right| D_{i}$ is the interstitial-sink (dislocation) reaction rate coefficient, $K_{v s}^{\alpha}=\left|z_{v}^{\alpha}\right| D_{v}$ is the vacancy-sink (dislocation) reaction rate coefficient. Note that the rate of defect absorption at mobile dislocation sinks (per slip system) also corresponds to the flux of point defects causing climb of mobile dislocations (cf. Section 2.3.3.2.2). Implications will be explained in the next section. The defect pair creation and recombination rates are assumed to be constant over the entire deformation process. This formulation is based on a standard rate theory approach used previously to model the evolution of point defects $[60,61]$. 


\subsection{Interstitial loops}

Rate equations for the evolution of interstitial loops have been developed in the past, which are based on distributing the loop population into different size classes [62,63]. A rate theory-based model for similar conditions of loop growth due to dislocation climb was developed by Semenov et al. [64], again using a distribution of the dislocation loops with different size classes. Implementation of such models in a crystal plasticity framework is deemed impractical.

Here we propose a simple model, assuming that interstitial loops are formed when the mobile edge dislocation segments climb by absorbing point defect clusters. It follows that the climb rate of mobile dislocations will yield the rate of formation of interstitial loops, while the absorption rate of point defects at dislocations will yield the growth rate of the loops. All interstitial loops on slip plane $\alpha$ are assumed to be of mean size $d_{i}^{\alpha}$. It is also assumed that all loops are planar and approximately circular in shape. Recall that in Section 2.3.3.2.3, the rate of

migration of mobile edge dislocations due to climb is given by $\dot{\rho}_{c \lim b}^{\alpha}=\left(\left|v_{c}^{\alpha}\right| / l_{c}\right) \rho_{M}^{\alpha}=\left|\dot{\gamma}_{c}^{\alpha}\right| / b l_{g}$. This population of mobile dislocation segments grows into interstitial loops by absorption of interstitials. Conserving the total line length of mobile dislocations involved in climb, we arrive at $\pi \dot{N}_{i}^{\alpha} d_{i}^{\alpha}=\dot{\rho}_{c \lim b}^{\alpha}$. The rate of creation of new interstitial loops is therefore given by

$$
\dot{N}_{i, c \lim b}^{\alpha}=\frac{1}{\pi d_{i}^{\alpha}} \frac{\left|\dot{\gamma}_{c}^{\alpha}\right|}{b l_{g}}
$$

The dependence of interstitial loop size as a function of the number of interstitials absorbed per loop is not entirely known. In this model, the areal growth rate of the planar loops is assumed to be a linear function of the number of interstitials absorbed per loop. As will be shown later, this assumption gives a reasonable estimate of the loop size evolution, at least for low radiation doses. From Section 2.3.3.3.1, the rate of absorption of interstitials per slip system is given as $K_{i s}^{\alpha} \rho_{M}^{\alpha} c_{i}$. Hence, if we know the number of interstitials, $i^{0}$, required to form a loop of area, $A_{i}^{0}$, the average areal growth rate of $N_{i}^{\alpha}$ loops can be given as

$$
\dot{A}_{i}^{\alpha}=\frac{1}{N_{i}^{\alpha}} \frac{A_{i}^{0}}{i^{0}} K_{i s}^{\alpha} \rho_{M}^{\alpha} c_{i}
$$

Furthermore, $A_{i}^{\alpha}=\pi / 4 d_{i}^{\alpha 2}$. This leads us to the growth rate for the size of interstitial loops, i.e.,

$$
\dot{d}_{i, \text { growth }}^{\alpha}=\frac{2}{\pi} \frac{A_{i}^{0}}{i^{0}} \frac{K_{i s}^{\alpha} \rho_{M}^{\alpha} c_{i}}{N_{i}^{\alpha} d_{i}^{\alpha}}
$$

The parameters $i^{0}$ and $A_{i}^{0}$ need to be estimated from experimental data. Note that vacancy loops could be formed by a similar mechanism. However, the diffusivities of vacancies 
are much lower than that of interstitials, leading to a comparatively lower absorption rate at dislocations. These loops are therefore relatively smaller than the corresponding interstitial loops and do not cause considerable impediment to glide. Hence, for simplicity, we do not track the evolution of vacancy loops in our simulations. The absorption of vacancies at dislocations is however accounted for in Eq. (2.10).

The models discussed here are essentially based on creation-exchange relations, with defects being created, recombined, or transformed from one type to another. For simplicity, we have neglected considering the full character of transport relations (defect concentration gradients and fluxes). We will comment on implications of this idealization in later discussion.

\subsection{Dislocation densities}

This section discusses the evolution of the mobile and immobile components of dislocation densities on each slip system. Various laws have been proposed to statistically represent the substructure evolution for specific deformation processes. Here we extend these laws to model inelastic deformation in irradiated bcc materials.

Dominant mechanisms governing substructure evolution in bcc irradiated materials can be listed as follows: generation of new dislocations from pre-existing dislocation segments ( $\dot{\rho}_{m u l}^{\alpha}$ ), migration of mobile dislocations by cross-slip $\left(\dot{\rho}_{c s}^{\alpha}\right)$ and climb $\left(\dot{\rho}_{c \operatorname{limb}}^{\alpha}\right)$, mutual annihilation of mobile dislocations $\left(\dot{\rho}_{\text {mutual }}^{\alpha}\right)$, trapping of mobile dislocations at barriers, rendering them immobile $\left(\dot{\rho}_{\text {trap }}^{\alpha}\right)$, and dynamic recovery of immobile dislocations by thermally activated processes $\left(\dot{\rho}_{d y n}^{\alpha}\right)$. Each of these mechanisms are explained next.

\section{Multiplication of mobile dislocations}

The rate of formation of new dislocation segments is a function of the area swept on the slip plane by gliding mobile dislocations. This relation proposed by Essmann and Mughrabi [65] is of the form

$$
\dot{\rho}_{m u l}^{\alpha}=\frac{k_{m u l}}{b l_{d}}\left|\dot{\gamma}^{\alpha}\right|
$$

where $l_{d}$ is the average line length of the dislocation segment and $k_{m u l}$ is a material constant. Further, $l_{d}$ is inversely related to the current mobile dislocation density, i.e., $l_{d} \approx 1 / \sqrt{\sum_{\varsigma} \rho_{M}^{\varsigma}}$. Thus, we can write the rate of formation of mobile dislocations as

$$
\dot{\rho}_{m u l}^{\alpha}=\frac{k_{m u l}}{b} \sqrt{\sum_{\varsigma} \rho_{M}^{\varsigma}}\left|\dot{\gamma}^{\alpha}\right|
$$




\section{Cross-slip of mobile screw dislocations}

Cross-slip of screw dislocations is a prominent process in bcc metals, because of the existence of several slip planes on to which a screw dislocation with a unique Burgers vector can migrate. Cross-slip helps bypass barriers to glide and reduce internal stresses in the material. At low temperatures, a $\langle 111>$ type screw dislocation may spread to three $\{110\}$ and three $\{112\}$ slip planes. At higher temperatures, six more $\{123\}$ planes come into the picture for a given slip direction. This kind of dislocation migration along a specific slip direction, but spread across multiple slip planes, is commonly referred to as wavy slip. A simple stochastic model for crossslip is proposed here. Cross-slip of screw dislocations in bcc materials is known to occur by the double kink mechanism [66]. Cross-slip is a thermally activated process; the probability that a screw dislocation will cross-slip to another slip plane $\zeta$ is proportional to:

$$
P^{\zeta} \propto \exp \left(-\frac{\left(\tau^{*}-\left|\tau^{\zeta}\right|\right) V_{a}}{k T}\right)
$$

where $\tau^{*}$ is the critical activation stress for double kink nucleation, $V_{a}$ is the corresponding activation volume [67], and $\tau^{\zeta}$ is the resolved shear stress on slip system $\zeta$.

Consider a subset $\Psi$ of slip systems, such that the slip direction is the same for all slip systems in subset $\Psi$. The probability of cross-slip is calculated on each of the slip planes belonging to $\Psi$. It is assumed that all screw dislocations belonging to $\Psi$ will then cross-slip to the plane $\zeta$ having the highest probability, i.e., $P^{\zeta}=\max \left(P^{\alpha}\right) \forall \alpha, \zeta \in \Psi$. Accordingly, we calculate the associated dislocation annihilation rate on slip system $\alpha$ of subset $\Psi$ as

$$
\dot{\rho}_{c s}^{\alpha}=-k_{c s} \exp \left(-\frac{\left(\tau^{*}-\left|\tau^{\zeta}\right|\right) V_{a}}{k T}\right) \rho_{M}^{\alpha}\left|\dot{\gamma}^{\alpha}\right|
$$

where $k_{c s}$ is a material constant. This would result in additional dislocations on slip system $\zeta$ accumulating from the rest of the slip systems of subset $\Psi$, i.e.,

$$
\dot{\rho}_{c s}^{\zeta}=-\sum_{\alpha \in \Psi, \alpha \neq \zeta} \dot{\rho}_{c s}^{\alpha}=\sum_{\alpha \in \Psi, \alpha \neq \zeta} k_{c s} \exp \left(-\frac{\left(\tau^{*}-\left|\tau^{\zeta}\right|\right) V_{a}}{k T}\right) \rho_{M}^{\alpha}\left|\dot{\gamma}^{\alpha}\right|
$$

Note that slip plane $\zeta$ with the highest probability for cross-slip also corresponds to the highest absolute resolved shear stress, i.e., the slip plane that is most favorably oriented with respect to the imposed stress state. Dislocation glide on slip plane $\zeta$ would thus be comparatively easier.

\section{Climb of mobile dislocations}


As mentioned earlier, climb is associated with the migration of mobile dislocations. The model for dislocation climb was given in Section 2.3.3.2.3, and the associated rate of migration of mobile dislocations is of the form

$$
\dot{\rho}_{c \lim b}^{\alpha}=\frac{\left|\dot{\gamma}_{c}^{\alpha}\right|}{b l_{g}}
$$

\section{Mutual annihilation of mobile dislocations}

Mutual annihilation of edge dipoles and mobile screw dislocation segments with opposite Burgers vectors occurs when dislocation segments lie within a critical capture radius, $R_{c}$ [68], i.e.,

$$
\dot{\rho}_{\text {mutual }}^{\alpha}=\frac{2 R_{c}}{b} \rho_{M}^{\alpha}\left|\dot{\gamma}^{\alpha}\right|
$$

The factor of two accounts for the fact that two dislocations with opposite Burgers vectors are annihilated as a result of this mechanism.

\section{Trapping of mobile dislocations}

Mobile dislocations get trapped at barriers on the slip plane and the corresponding dislocation segments are immobilized. For the case of irradiated materials, these barriers are in the form of dislocation networks (forest interactions) and interstitial loops. Based on a statistical model for trapping $[69,70]$, the dislocation trapping rate is given by

$$
\dot{\rho}_{\text {trap }}^{\alpha}=\frac{1}{b \lambda^{\alpha}}\left|\dot{\gamma}^{\alpha}\right|
$$

where $\lambda^{\alpha}$ is the effective free path of dislocation trapping on slip system $\alpha$. Since this expression gives a rate for immobilization of mobile dislocations, the immobile dislocation density would increase at the same rate. The effective free path is generally estimated as the harmonic mean of individual barrier free paths [71], i.e.,

$$
\frac{1}{\lambda^{\alpha}}=\frac{1}{\lambda_{d i s}^{\alpha}}+\frac{1}{\lambda_{\text {int }}^{\alpha}}
$$

where $\lambda_{\text {dis }}^{\alpha}$ and $\lambda_{\text {int }}^{\alpha}$ are the mean free paths of the dislocation networks and interstitial loops, respectively. For dislocations, the mean free path has an inverse dependence on the square root of the defect density [70]. The effective free path can therefore be written as

$$
\frac{1}{\lambda^{\alpha}}=\beta_{\rho} \sqrt{\rho^{\alpha}}+\beta_{i} \sqrt{N_{\mathrm{i}}^{\alpha} d_{\mathrm{i}}^{\alpha}}
$$

where $\beta_{\rho}$ and $\beta_{\mathrm{i}}$ are associated material constants.

\section{Dynamic recovery of immobile dislocations}

Dynamic recovery of immobile dislocations due to thermally activated processes is considered in a single rate expression [72], i.e., 


$$
\dot{\rho}_{d y n}^{\alpha}=k_{d y n} \rho_{I}^{\alpha}\left|\dot{\gamma}^{\alpha}\right|
$$

where $k_{d y n}$ is a material constant. Assembling terms, the defect densities and sizes evolve by the following relations:

$$
\begin{gathered}
\dot{\rho}_{M, \text { evolution }}^{\alpha}=\dot{\rho}_{\text {mul }}^{\alpha}-\dot{\rho}_{c s}^{\alpha}-\dot{\rho}_{c \lim b}^{\alpha}-\dot{\rho}_{\text {mutual }}^{\alpha}-\dot{\rho}_{\text {trap }}^{\alpha} \\
\dot{\rho}_{I, \text { evolution }}^{\alpha}=\dot{\rho}_{\text {trap }}^{\alpha}-\dot{\rho}_{d y n}^{\alpha} \\
\dot{N}_{i}^{\alpha}=\dot{N}_{i, \text { lim } b}^{\alpha} \\
\dot{d}_{i}^{\alpha}=\dot{d}_{i, \text { growth }}^{\alpha}
\end{gathered}
$$

Note that the interaction of dislocations with interstitial loops has been considered only in terms of its contribution to the hardening behavior (Eq. (2.5)), and trapping of immobile dislocations thus rendering them immobile (Eq. (2.23)). Physically, the interaction of interstitial loops with glide dislocations is expected to reduce the density of the loops and cause flow localization along dislocation channels [26,73-75]. Models to capture this effect will be discussed in Chapter 3. Some parameter estimation is required in these evolution models to fit the experimental data.

\subsection{Model implementation}

The constitutive model developed herein is applied to model quasi-static tension and creep responses of a Mod (modified) 9Cr-1Mo steel. Mod 9Cr-1Mo steel is a ferritic/martensitic steel that has potential high temperature nuclear applications, such as the cladding in fast reactors. The material is subject to considerable radiation damage in these applications.

\subsubsection{Parameter estimation}

Temperature dependence of the elastic constants is taken from Ref. [76]. Apart from the elastic constants, not much material-specific data are available for estimating the model parameters. Mod 9Cr-1Mo steel contains mostly ferritic and martensitic phases. This steel derives its strength primarily from lath martensite, which has a body centered tetragonal (bct) crystal structure. Subsequent tempering leads to a tempered martensite phase which is essentially body centered cubic [77]. It is thus reasonable to assume that the material has bcc crystal structure with 48 slip systems.

Model parameters relying on actual physical data were estimated from the corresponding

values of (bcc) alpha-Fe. The Burgers vector magnitude is that of bcc-Fe, $b=(\sqrt{3} / 2) a_{0}$, where $a_{0}=0.2866 \mathrm{~nm}$ is the lattice parameter of bcc-Fe [78]. Model parameters were calibrated to fit the experimental stress-strain response of virgin (unirradiated) material and then used to simulate 
tensile test behavior under irradiation. The following explains the rationale behind choosing specific parameter values. The values of all the parameters are listed in Table 1.

\subsubsection{Dislocation glide}

Ashby [79] suggested the activation energy for dislocation glide, $\Delta F_{g}$, to be of the order of $0.5 G b^{3}$ for irradiated materials. In our calculations, the activation energy was estimated to be $0.35 G b^{3}$ based on fit to available experimental tension test data. Further, the shape parameters $p$ and $q$ of the enthalpy curve should lie in the range $0<p<1,1<q<2$. The values, $p=0.28$, and $q=1.34$, adopted for bcc-Ta by Kothari and Anand [48] are found to fit our data reasonably. Temperature and strain rate dependence of the cooperative attempt frequency for dislocation glide, $v$, is not exactly known, but it is typically of the order $v \approx 10^{10}-10^{11} \mathrm{~s}^{-1}$. Further the mobile dislocation density $\rho_{M}^{\alpha} \approx 10^{7} \mathrm{~mm}^{-2}$, and the mean dislocation glide distance $l_{g} \approx 15-20 \mathrm{~nm}$. Accordingly, the pre-exponential factor, $\dot{\gamma}_{0}=\rho_{M}^{\alpha} b l_{g} v$, for the dislocation glide relation, $\dot{\gamma}_{g}^{\alpha}=\rho_{M}^{\alpha} b v_{g}^{\alpha}=\dot{\gamma}_{0} v_{g}^{\alpha} /\left(l_{g} v\right)$, is assumed to have a constant value of $4.0 \times 10^{5} \mathrm{~s}^{-1}$ in our simulations.

In this work, the Peierls barrier stress has been assumed to be constant over all simulation temperatures and strain rates. Although this may not hold over the entire temperature regime, predictions are limited by lack of experimental data beyond $400 \mathrm{~K}$ for bcc-iron. Beyond $400 \mathrm{~K}$, although the dependence of flow stress is not expected to be dominated by the Peierls barrier, it is also unlikely that the flow stress will be entirely independent of the Peierls barrier. As suggested in the literature [80], a value of $390 \mathrm{MPa}$ has been used for the Peierls barrier.

The barrier strengths for dislocations, $q_{\rho}$, and interstitial loops, $q_{i}$, were calibrated to the experimental data and found to have the values $q_{\rho}=0.3, q_{i}=0.4$. These values are reasonably close to the barrier strengths used by Arsenlis et al. [12] for irradiated fcc-Cu. The coefficients of dislocation interaction ( $A^{\alpha \zeta}$ ) among all the 48 slip systems in bcc are not as well characterized as for fcc crystals. We have used a value of $A^{\alpha \alpha}=1.0$ for the self-interaction coefficient and $A^{\alpha \xi}(\xi \neq \alpha)=0.2$ for the latent interaction coefficient. This is based on the assumption that the strongest resistance to glide on a slip plane will be offered by dislocations on the plane itself, and latent interaction offers a relatively weaker resistance. This value of latent interaction coefficient is of the same order as that predicted by Queyreau et al. [81] for different types of interactions and dislocation junction formations in alpha-Fe, based on DD simulations. 


\subsubsection{Dislocation climb}

Estimation of dislocation climb parameters requires knowledge of the diffusivities of self-interstitial atoms (SIAs) and vacancies in the material. Point defect diffusivities depend on the migration energies of the respective defects, i.e.,

$$
D_{p}=D_{p, 0} \exp \left(-\frac{E_{p}^{m}}{k T}\right)
$$

where $E_{p}^{m}$ is the migration energy and $D_{p, 0}$ is the diffusion constant of point defect type $p$. The corresponding values for SIAs and vacancies are based on MD simulations for bcc-Fe performed by Mendelev and Mishin [82]. The proportionality constants for interstitial and vacancy capture efficiencies, $z_{i}^{0}$ and $z_{v}^{0}$, were estimated from fit to experimental data. It has however been suggested in rate theory calculations performed by Stoller et al. [83] that the capture efficiency for self-interstitials is roughly 1.2 times that for vacancies, and this ratio is maintained in our calculations. The average dislocation glide distance between two barriers, $l_{g}$, is estimated (from the mean free path of the dislocations) to be $15 \mathrm{~nm}$. This value is assumed to be constant in our simulations, since the average glide distance remains in the same range for dislocation densities evolving over the entire deformation domain. Further, the average dislocation climb distance $l_{c}$ is assumed to be $3 b$. This is reasonable considering the fact that the barriers to glide present in the form of planar loops and dislocation networks, can be overcome by climbing a few atomic planes with respect to the slip plane. This would have been different for the case of larger obstacles, such as precipitates, where the climb distance would be much higher.

\subsubsection{Evolution of defects}

\subsection{Point defects}

The defect pair creation rate, $K_{0}$, is a function the incident radiation flux and the probability of atomic displacement due to radiation. Consequently, $K_{0}$ depends on the energy of the incident particles (neutrons, ions, etc.). The underlying physics is beyond the scope of the current work. However, based on calculations done in [6], it is estimated that $K_{0}$ is typically of the order $10^{12}-10^{13} \mathrm{~mm}^{-3} \mathrm{~s}^{-1}$ for neutron irradiation in steels. In our simulations, a value of $K_{0}=2.5 \times 10^{12} \mathrm{~mm}^{-3} \mathrm{~s}^{-1}$ is used for the creep tests, unless specified otherwise. It has been suggested that in the high temperature, high sink (dislocations) density regime, contribution of the defect pair recombination rate, $K_{i v}$, is negligible compared to the other evolution terms in the rate equations [6]. This term is therefore set to zero in our simulations. 
Table 1. Model parameters for Mod 9Cr-1Mo steel.

\begin{tabular}{lll}
\hline Parameter & Value & Meaning \\
\hline$E, v$ & $207300-64.58(\mathrm{~T}[\mathrm{~K}]-273) \mathrm{MPa}, 0.3$ & Elastic constants \\
$a_{0}$ & $0.2866 \mathrm{~nm}$ & Lattice parameter \\
$\Delta F_{g}, p, q, \dot{\gamma}_{0}$ & $0.35 G b^{3}, 0.28,1.34,4.0 \times 10^{5} \mathrm{~s}^{-1}$ & Flow rule parameters \\
$\tau_{p}, q_{\rho}, q_{i}, A^{\alpha \alpha}$, & $390 \mathrm{MPa}, 0.3,0.4,1.0,0.2$ & Hardening parameters \\
$A^{\alpha \varsigma}(\varsigma \neq \alpha)$ & & \\
$z_{i}^{0}, z_{v}^{0}, \Omega, l_{g}, l_{c}$ & $1.2 \times 10^{-4}, 1.0 \times 10^{-4}, b^{3}, 1.5 \mathrm{~nm}, 3 b$ & $\begin{array}{l}\text { Dislocation climb } \\
\text { parameters }\end{array}$ \\
$K_{i v}, A_{i}^{0}, i^{0}$ & $0,12.57 \mathrm{~nm}^{2}, 91$ & $\begin{array}{l}\text { Point defect and } \\
\text { interstitial loop }\end{array}$ \\
evolution parameters \\
$D_{i, 0}, D_{v, 0}, E_{i}^{m}, E_{v}^{m}$ & $5.34 \times 10^{-2} \mathrm{~mm}^{2} \mathrm{~s}^{-1}, 7.87 \times 10^{-1} \mathrm{~mm}^{2} \mathrm{~s}^{-1}$, & $\begin{array}{l}\text { Diffusivities and } \\
\text { migration energies }\end{array}$ \\
$k_{m u l}($ quasi-static $)$, & $0.143,1.02,329.5,1 \mathrm{~nm}, 0.36,0.3$ & $\begin{array}{l}\text { Dislocation evolution } \\
\text { parameters }\end{array}$ \\
$k_{m u l}($ creep $), k_{d y n}$, & & \\
$R_{c}, \beta_{\rho}, \beta_{i}$ & $8.0 \times 10^{3}, 0.004 G, 20 b^{3}$ & \\
$k_{c s}, \tau^{*}, V_{a}$ & & Cross-slip parameters \\
\hline
\end{tabular}

\subsection{Interstitial loops}

As mentioned earlier, the areal growth rate of interstitial loops is assumed to be a linear function of the interstitials absorbed per loop. Marian et al. [84] characterized the self-interstitial loops in bcc iron and found that a $4 \mathrm{~nm}$ loop contains 91 SIAs. Though the number of interstitials per loop has been found to be lower for larger (>10 nm) loops, it is reasonable to assume this ratio to be constant over a limited range of cumulative radiation damage. Accordingly, a value of $i^{0}=91$ and $A_{i}^{0}=12.57 \mathrm{~nm}^{2}$ is used in our simulations. These approximations can be appropriately modified for higher dpa levels. 


\subsection{Dislocation densities}

Dislocation evolution parameters in the model required were calibrated to fit the experimental stress-strain curves as the precise quantitative evolution behavior of these substructure variables is not known.

The dislocation multiplication model is essentially a phenomenological rule based on experimental observations of the growth of dislocation loops [65]. It has been found from our simulations that the dislocation multiplication constant $k_{m u l}$ should have a higher value in creep tests as compared to quasi-static tension tests. A higher multiplication rate in creep tests is needed to account for the reduction in dislocation density due to climb of mobile dislocations by absorption of point defects. As will be explained later, it is assumed that point defects due to radiation are produced dynamically in the material only during the creep tests (i.e., continuous irradiation), and accordingly the dislocation multiplication rate needs to be higher in these simulations. The dislocation multiplication constant used for tension tests has a value $k_{m u l}=0.143$ which is in the same range as the value used by Evers et al. [85], i.e., $k_{m u l}=0.1$, for similar simulations of polycrystalline $\mathrm{Cu}$. For the creep tests, however, a higher value of $k_{m u l}=1.02$ is used. Higher values of the constant are also justified by the elevated temperatures at which creep experiments are performed, leading to a higher rate of multiplication of mobile dislocations.

To the best of our knowledge there are no quantitative studies on the evolution rate of dislocations due to cross-slip, in a statistical sense. Hirthe and Lothe [66] calculated the critical stress, $\tau^{*}$, required by a dislocation for double kink nucleation and this was plotted as a function of the dislocation line length, $l_{d}$, in the work of Rhee et al. [67]. Given an average mobile dislocation density of $\approx 2 \times 10^{7}-3 \times 10^{7} \mathrm{~mm}^{-2}$ per slip system, $l_{d} \approx 100 \mathrm{~b}-150 \mathrm{~b}$. The calculations of Rhee et al. show that in this range $\tau^{*} \approx 0.004 G$. In the present model we have used the above value, assuming it to be constant over the entire range of dislocation densities. Further, the activation volume for cross-slip is assumed to be $V_{a}=20 b^{3}$. Estimation of the amount of crossslip on each slip system in a polycrystal ensemble is not exactly trivial and the value of the material constant, $k_{c s}$, has been calibrated such that the rate of cross-slip is comparable to the other dislocation evolution mechanisms at higher stresses and elevated temperatures.

For the mutual annihilation of dislocations, a capture radius, $R_{c}=1 \mathrm{~nm}$, is used. This value is of the same order as that used by Arsenlis et al. [12] for irradiated fcc copper $\left(R_{c}=8.5 b\right.$ ). The trapping coefficients for dislocations and interstitial loops and the dynamic recovery constant are calibrated based on fit to the experimental data. 


\subsubsection{Measure of radiation damage}

Radiation damage is conventionally measured in terms of cumulative effective displacements per atom (dpa) in the material based on the flux of incident particles. Calculation of the effective atomic displacements is however infeasible in the present model, in a continuum sense. Quantitative measure of radiation damage is therefore done in the present model using a correlation for interstitial loop density and size to the cumulative dpa damage based on experimental observations. Deo et al. [50] proposed a square root dependence on cumulative dpa for similar materials for both the loop density and size, i.e.,

$$
\begin{aligned}
& N_{i}=A \cdot \mathrm{dpa}^{-1 / 2} \\
& d_{i}=B \cdot \mathrm{dpa}^{-1 / 2}
\end{aligned}
$$

where $A=5 \times 10^{13} \mathrm{~mm}^{-3}$ and $B=3.7 \times 10^{-6} \mathrm{~mm}$. The same is used in our simulations. Note that this is an empirical relation fit to experimental observations of the variation of loop density and size over a limited range of dpa damage, and will not hold for high $(>10 \mathrm{dpa})$ or very low $(<1$ dpa) radiation doses. Further, the interstitial loops are assumed to be uniformly distributed in the material. Effects of irradiation temperature, material depth from irradiated surface, etc. are neglected here as beyond the scope of the present work.

\subsection{Results and discussion}

\subsubsection{Quasi-static tension tests}

Three-dimensional displacement-controlled simulations were performed using the finite element program ABAQUS [86] on 125 cubic grain ensembles, with $30 \mu \mathrm{m}$ sized grains that have random crystallographic orientation distribution. The constitutive equations were implemented using the User MATerial subroutine (UMAT) in ABAQUS. Periodic boundary conditions were enforced in all directions; implying that displacements on opposite boundaries are tied to each other [87].

It is assumed that all tensile test samples are pre-irradiated and there is no concurrent radiation damage during the tension tests. This is reasonable considering the fact that samples are generally irradiated at the rate of $\approx 10^{-6}-10^{-5} \mathrm{dpa}-\mathrm{s}^{-1}$; radiation damage over the time-scales of the tension tests $(\approx 1000 \mathrm{~s})$ will be negligible compared to the damage already accumulated. In this work, simulation results are compared to the experimental data for Mod $9 \mathrm{Cr}-1 \mathrm{Mo}$ steel from Ref. [2]. It has been confirmed from experiments that Mod 9Cr-1Mo steel has very high dislocation densities of the order $\approx 10^{7}-10^{8} \mathrm{~mm}^{-2}$ [88,89]. Further, additional dislocations are generated in the material during the course of irradiation. Accordingly, initial dislocation densities of $\rho_{M}^{0}=2 \times 10^{7} \mathrm{~mm}^{-2}$ and $\rho_{I}^{0}=3 \times 10^{7} \mathrm{~mm}^{-2}$ are assumed on all 48 slip systems. 
Tension tests were conducted at a strain rate of $10^{-4} \mathrm{~s}^{-1}$. The results were calibrated with experimental data [2]. Figure 2 compares simulation results for true stress-strain behavior with experimental data. A reasonable fit for the stress-strain curves was obtained over various temperatures and radiation dose histories with a single set of parameters, up to and just beyond the point of material softening.

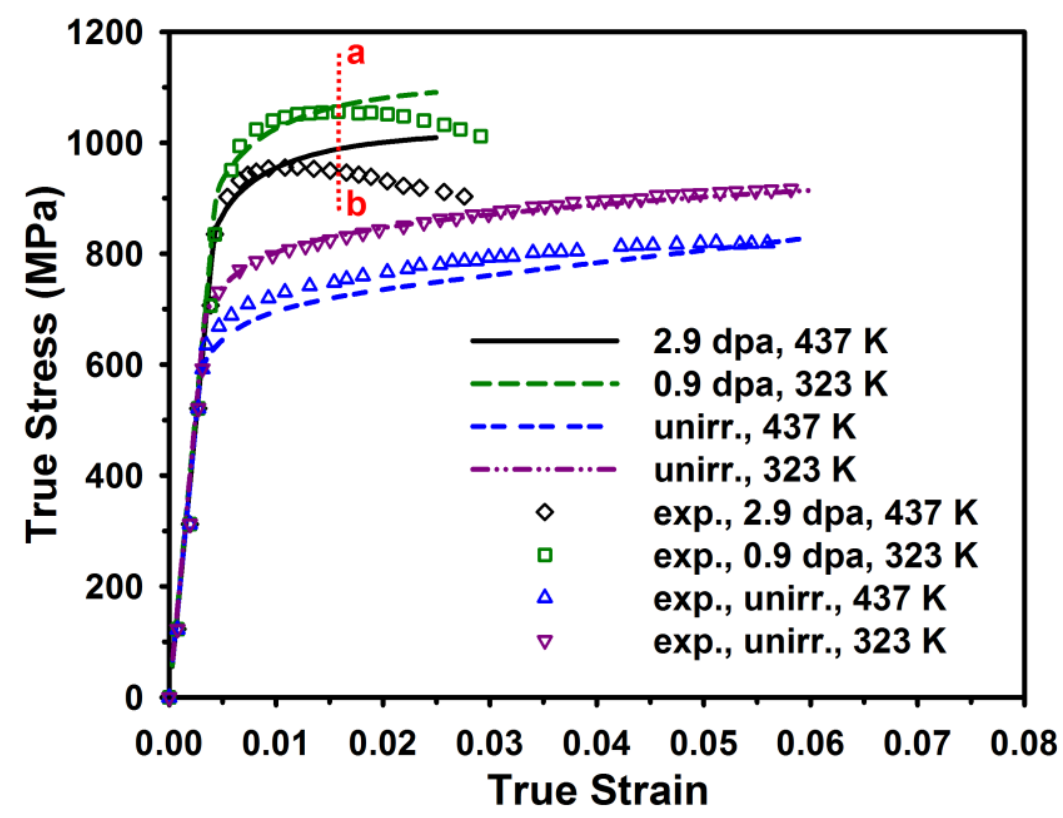

Figure 2. Comparisons of simulations calibrated to experimental stress-strain curves over a range of temperatures and dpa damage. Experimental data from [2]. Experimental data for true stress not corrected for necking. Dotted red line a-b indicates the strain up to which simulated stress-strain behavior for the irradiated samples can be reliable.

Beyond this point, however, the simulated stress-strain curves for the irradiated material have a less negative slope than the corresponding experimental results, and the simulated stressstrain curves appear to be diverging. Moreover, only the engineering stress-strain data were reported for the experiments, so conversion of the data past the point of diffuse necking instability is not reasonable for the virgin material. However, for the irradiated material, the post peak softening behavior in experiments has contributions both from the material softening associated with interplay of dislocations with irradiation-induced defects and attendant dislocation substructure evolution and the geometric softening due to the onset of diffuse necking. In the present formulation, interaction of dislocations with irradiation-induced defects has been addressed only in terms of irradiation hardening (cf. Eq. (2.5)), which contributes to the observed increase in initial yield stress. Beyond this, interaction of the irradiation-induced defects with glide dislocations is expected to cause a reduction in the density of the defects and 
create paths for easy dislocation glide [44,75]. Models to capture this material softening effect are discussed in Chapter 3.

Simulations regarding radiation dose-, temperature-, and strain rate-dependence of the tensile test behavior were also performed to parametrically explore model predictions. Results are shown in Figs. 3, 4, and 5, respectively. As expected, the initial yield stress increases with increasing radiation doses. The flow stress decreases with increasing test temperatures, and increases with increasing strain rates. Also note that at higher strain rates the increase in flow stress is not as pronounced. This is due to the fact that the strain rates used in these tests are still close to the quasi-static (strain-rate independent) regime.

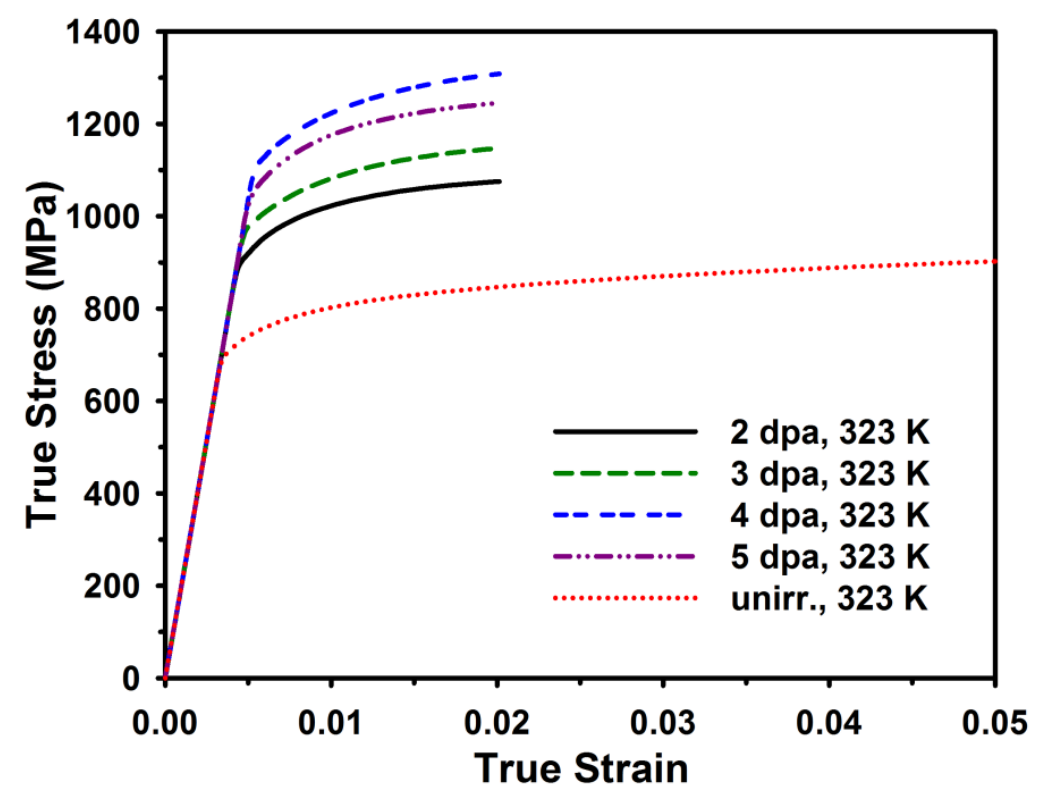

Figure 3. Effect of radiation dose on the stress-strain response. 


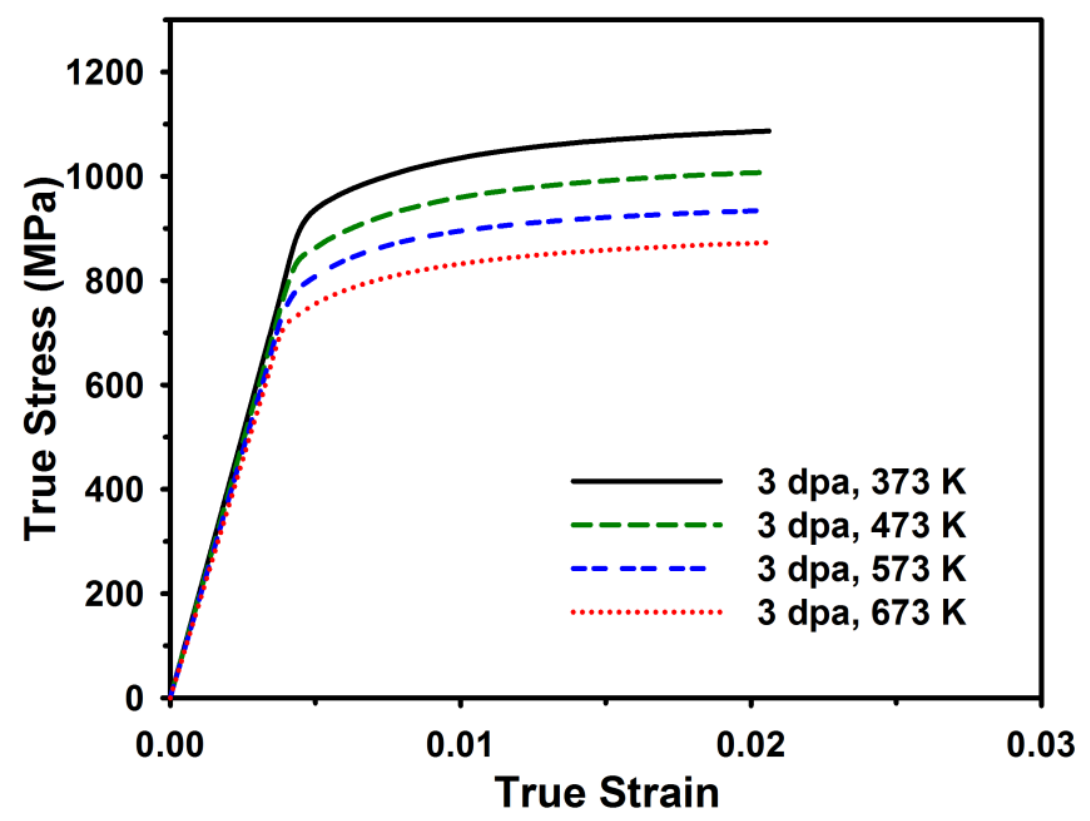

Figure 4. Effect of temperature on the stress-strain response.

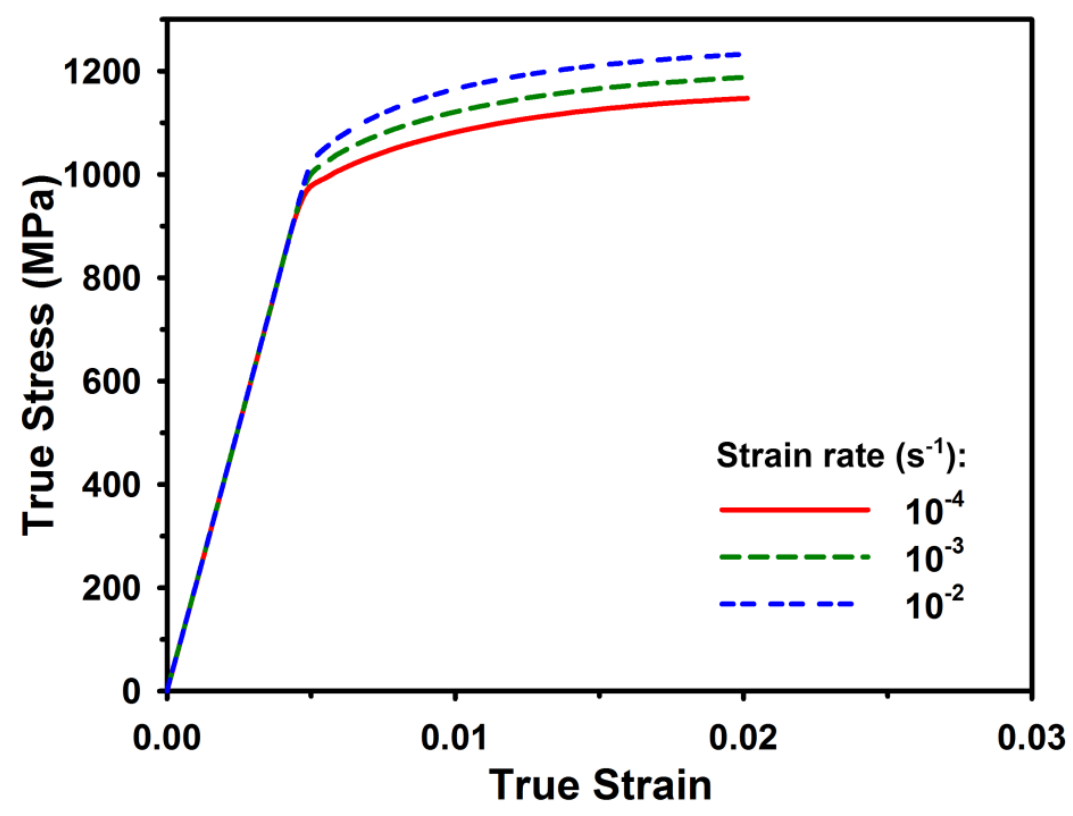

Figure 5. Effect of strain rate on the stress-strain response of the material loaded in tension at $323 \mathrm{~K}$ after being irradiated to $3 \mathrm{dpa}$. 


\subsubsection{Creep tests}

Creep simulations are computationally intensive due to the significantly longer timescales of these tests (100-1000 h). In this work, accelerated creep simulations were performed over shorter durations of time $(20-30 \mathrm{~h})$ to explore initial trends of the model under creep loading. They are accelerated in the sense that the rate of absorption of point defects, and consequently the climb rates of mobile dislocations are higher in our simulations. Threedimensional stress-controlled simulations were performed on 27 grain ensembles with $30 \mu \mathrm{m}$ cubic grains and random crystallographic orientation distribution. Periodic boundary conditions were used on the lateral (side) faces of the ensemble. All degrees of freedom were restricted on the top face. The bottom face was initially loaded in tensile stress to the requisite level, and the stress was then held constant for the desired duration of time.

It is assumed that the material has no prior deformation history, and has only mobile dislocations. Mobile dislocations will eventually be trapped and become immobile. Dislocation loops are also formed from the mobile dislocations. An initial dislocation density $\rho_{M}^{0}=1.0 \times 10^{8} \mathrm{~mm}^{-2}$ was assigned to each slip system. An initial concentration of $c_{i}^{0}=1.0 \times 10^{13} \mathrm{~mm}^{-3}$, and $c_{v}^{0}=1.0 \times 10^{13} \mathrm{~mm}^{-3}$ was used for the interstitials and vacancies, respectively. The initial point defect concentrations used are higher than the corresponding equilibrium concentrations (in the absence of irradiation). High initial concentrations have been used to avoid numerical instabilities in the simulations. Given the fact that the defect pair production rate is high during irradiation, these initial concentration levels used will be reached within seconds of the start of irradiation in actual experiments.

Simulations were performed over a range of stress, temperature and radiation damage rates. To the best of our knowledge, there is currently no representative experimental data in the literature to validate our simulation results. Predictive trends are consistent with expectations. Figure 6 shows the total strain evolution over time for different defect pair production rates. Of course, creep strain dominates after the initial loading portion. Simulations show that the creep rate depends linearly on the defect pair production rate. Based on the correlation between loop density and dpa levels in Eq. (2.30), the average radiation damage rate was found to be $1.05 \times 10^{-5} \mathrm{dpa}^{-1}$, and the corresponding average creep rate was $3.65 \times 10^{-8} \mathrm{~s}^{-1}$ at $160 \mathrm{MPa}$ true stress and $773 \mathrm{~K}$ for $K_{0}=2.5 \times 10^{12} \mathrm{~mm}^{-3} \mathrm{~s}^{-1}$. For the same stress and temperature conditions, when the defect production rate was increased to $K_{0}=5.0 \times 10^{12} \mathrm{~mm}^{-3} \mathrm{~s}^{-1}$, the radiation damage rate was found to be $2.12 \times 10^{-5} \mathrm{dpa}_{-\mathrm{s}^{-1}}$, and the corresponding creep rate was $7.31 \times 10^{-8} \mathrm{~s}^{-1}$. For $K_{0}=1.25 \times 10^{12} \mathrm{~mm}^{-3} \mathrm{~s}^{-1}$, the radiation damage rate was found to be $5.15 \times 10^{-6} \mathrm{dpa}-\mathrm{s}^{-1}$, and the corresponding creep rate was $1.57 \times 10^{-8} \mathrm{~s}^{-1}$. 


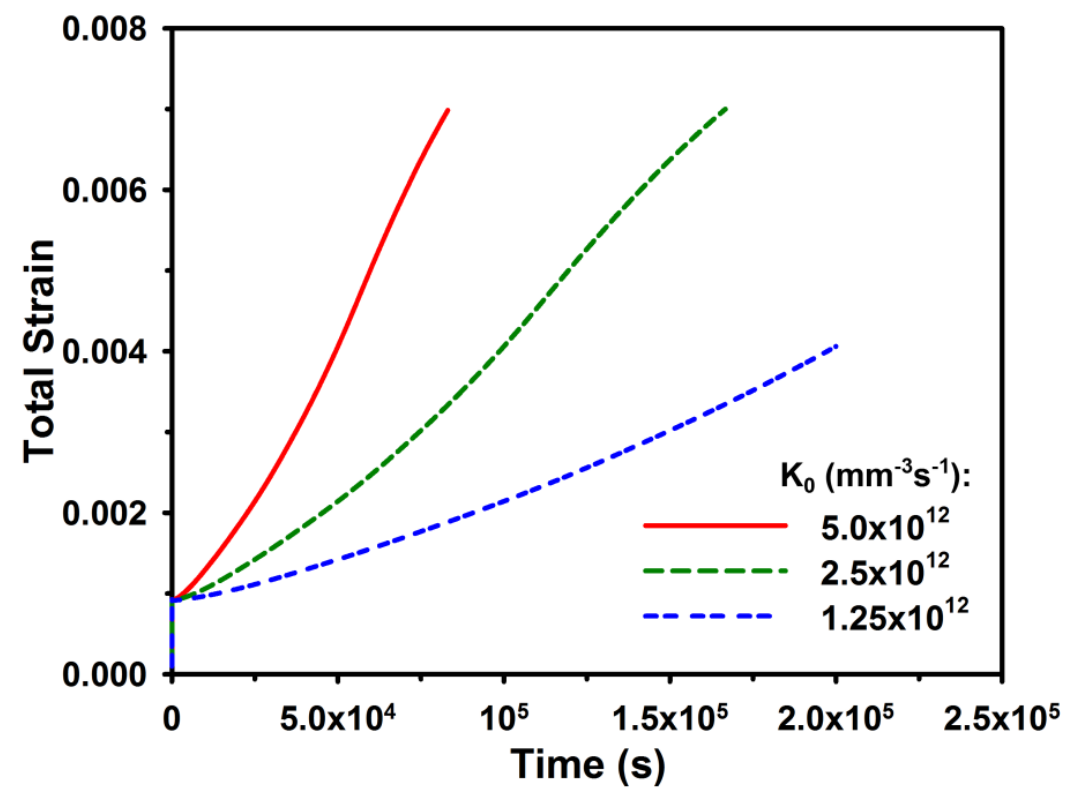

Figure 6. Total strain evolution over time for different defect pair production rates. The material was loaded to $160 \mathrm{MPa}$ in stress control at773 K.

Figure 7 shows the evolution of total strain over time for different true stress levels. The creep rate is initially dominated by SIPA. In the later stages however the creep rates start increasing. In the steady state creep regime, the mobile and the immobile dislocation evolution processes compete against each other, until the mobile dislocation density starts decreasing at a higher rate. This reduces the athermal slip resistance and facilitates dislocation glide, leading to higher creep rates during the later stages of deformation.

Figure 8 shows the evolution of defects (dislocation densities, areal interstitial loop density) during creep as a function of the total strain. The mobile and immobile dislocation evolution trends are (qualitatively) similar to those predicted by Magnusson et al. [90] for thermal creep. Note that the equilibrium vacancy concentration at $773 \mathrm{~K}$ is approximately $3.5 \times 10^{9} \mathrm{~mm}^{-3}$; as expected, the present vacancy concentration is of substantially non-equilibrium character. The mobile dislocation density decreases with increasing strain; and the immobile and areal interstitial loop densities increase with increasing creep. The areal interstitial loop density is however several times higher than the immobile dislocation density. This is due to the accumulation of defects associated with the radiation damage phenomena. Our simulations have also shown that the mobile dislocation densities decrease to very low levels on certain slip planes. The resolved normal stress, $\sigma_{c}^{\alpha}$, is higher on these planes, leading to a more rapid migration of mobile dislocations from them via absorption of point defects. 


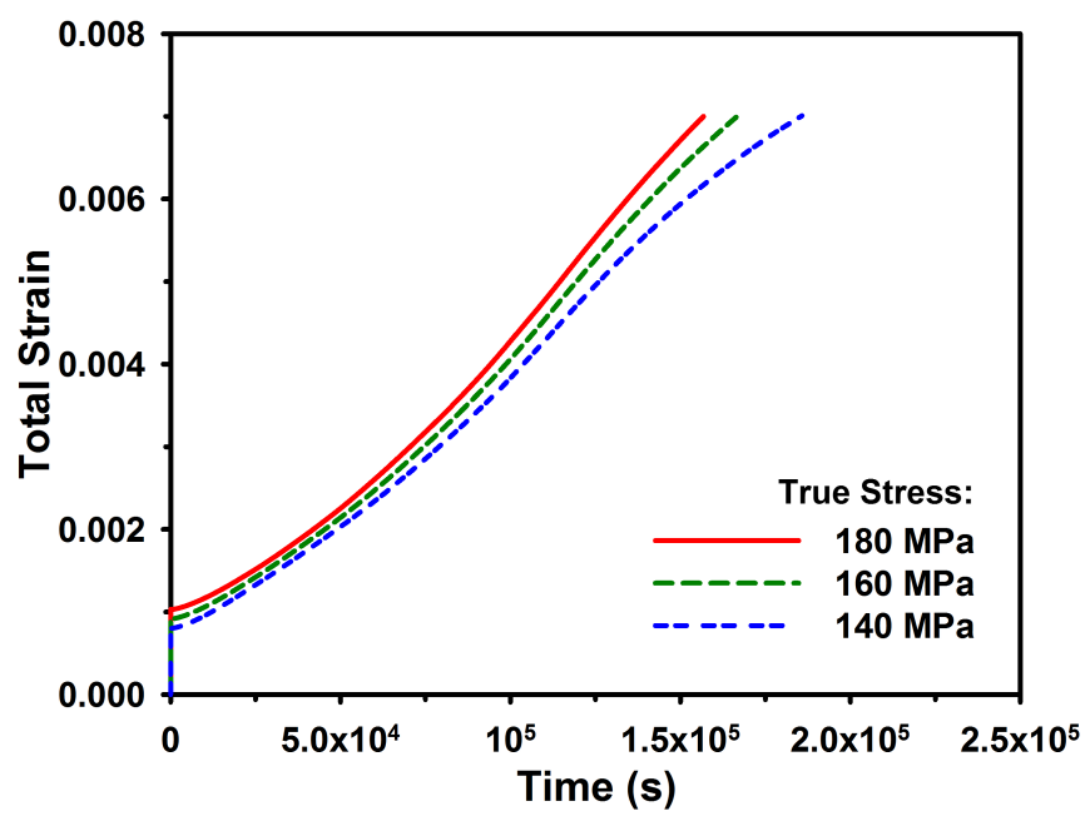

Figure 7. Total strain evolution over time for different stresses under creep loading at $773 \mathrm{~K} \cdot K_{0}=2.5 \times 10^{12} \mathrm{~mm}^{-3} \mathrm{~s}^{-1}$ in these simulations.

Figure 9 shows the evolution of point defects during creep as a function of the total strain. Qualitative trends compare well with the mean field rate theory calculations of Stoller et al. [83], at least initially. In the calculations of Stoller et al., a constant dislocation density was used. Note that they used a more complex rate theory formulation for point defect evolution, with terms accounting for additional mechanisms.

The spatial evolution of inelastic strain and defect substructure during irradiation creep deformation is discussed in detail in Chapter 5 . 


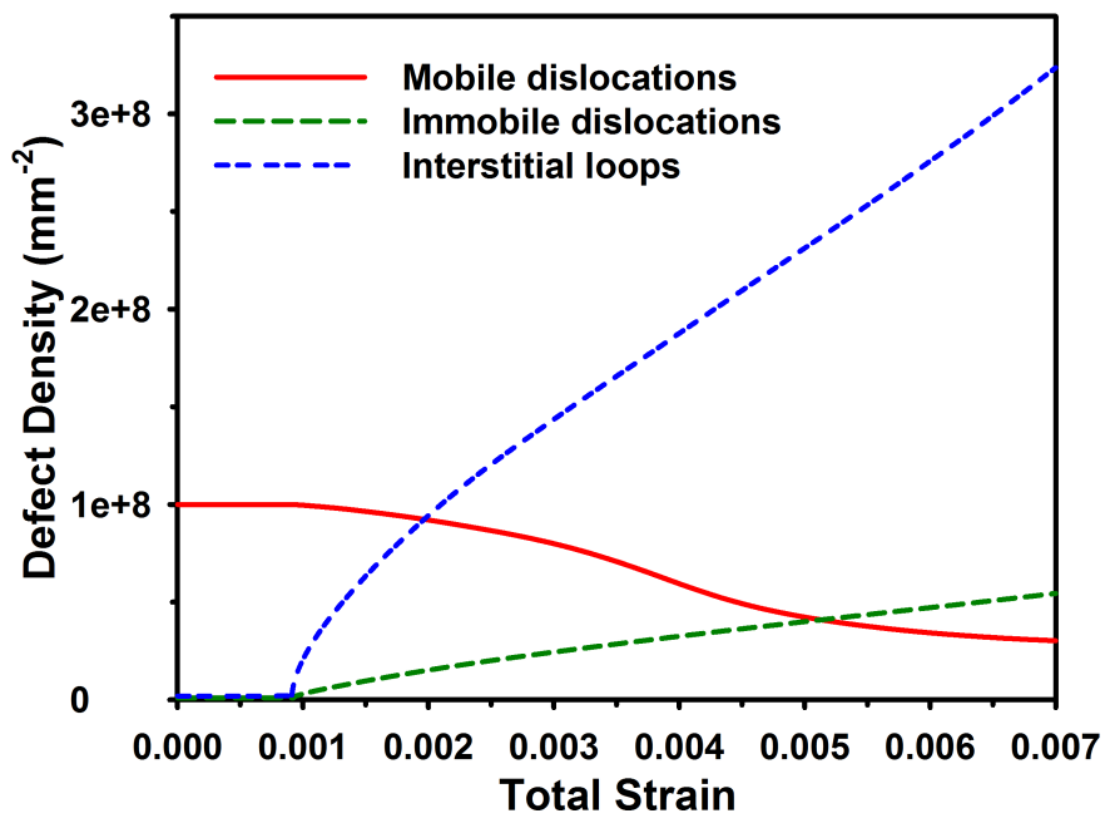

Figure 8. Average dislocation densities and areal interstitial loops density (per slip system) as a function of total strain. Initial threshold indicated loading of the sample in stress, before the constant stress creep regime has begun. The material was loaded to $160 \mathrm{MPa}$ at $773 \mathrm{~K} \cdot K_{0}=2.5 \times 10^{12} \mathrm{~mm}^{-3} \mathrm{~s}^{-1}$ in these simulations.

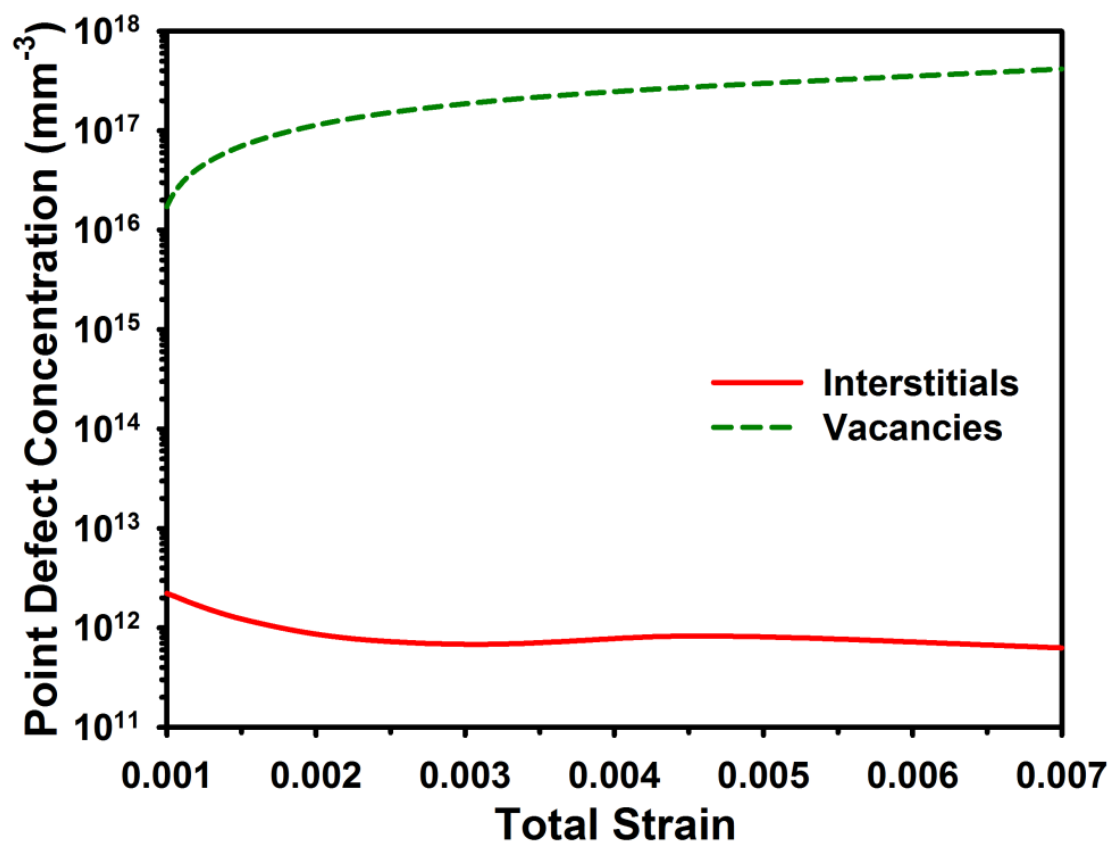

Figure 9. Average point defect concentrations as a function of total strain. Loading conditions same as in Fig. 8. 


\subsection{Summary}

A mechanism-based ISV continuum crystal plasticity model has been formulated to simulate the inelastic behavior of irradiated bcc structural materials. Statistically representative continuum models have been developed for cross-slip and climb of dislocations and growth of interstitial loops. A rate theory-based approach has been used to model the point defect evolution during radiation. The model was used to predict the quasi-static tensile behavior and creep behavior of Mod 9Cr-1Mo ferritic/martensitic steel. Reasonable calibration with available experimental quasi-static loading data for the initial hardening response was achieved and behavioral trends were predicted for creep loading. Most of the material parameters used in the model are based on physical constants and data. This mesoscale model provides a framework for bridging simulations across multiple length- and time-scales by drawing on information from various atomistic and kinetic Monte-Carlo simulations of radiation damage. The constitutive model has been proposed for bcc metals; however, it can be easily applied to other systems by making necessary modifications of the evolution mechanisms and relevant crystallography.

This Chapter lays the foundations of continuum framework to model the deformation behavior of irradiated bcc materials over a range of loading and radiation histories. The following Chapters will apply the framework to model these deformation histories, with necessary model modifications. 


\section{CHAPTER 3}

\section{MODELING LOCALIZED DEFORMATION DUE TO DISLOCATION \\ CHANNEL FORMATION ${ }^{2}$}

\subsection{Introduction}

The mechanical behavior of bcc ferritic/martensitic steels is significantly influenced by radiation damage, with effects including an increase in yield point, decrease in uniform ductility and considerably lower true strain to fracture [2,92-95]. Failure in these materials is governed by heterogeneous localized deformation due to the formation of dislocation channels, wherein majority of the inelastic strain is localized [26,73,74]. As a result, irradiated materials fail at relatively lower strains compared with virgin materials. In fact, the formation of dislocation channels and the resulting localized deformation is not just a feature of these ferritic/martensitic steels, but is observed in all irradiated metallic systems [96-99]. Research is therefore directed at understanding the physical mechanisms underlying these processes and the factors influencing them.

This Chapter focuses on modeling localized deformation in irradiated bcc materials using a mesoscale crystal plasticity model. A physics-based continuum constitutive framework was developed in Chapter 2 to model the quasi-static tensile and creep behavior of irradiated bcc metals. This framework is applied here to model the mechanical response and microstructure evolution resulting from heterogeneous localized deformation in irradiated bcc ferritic/martensitic steels. Necessary modifications are made to the existing constitutive model to capture the localization behavior.

\subsection{Background}

The microstructure of as-irradiated bcc metals is typically characterized by defect clusters and dislocation loops (primarily interstitial-type) [24,100,101]. On being subjected to tensile deformation, cellular structures of dislocations are observed at low radiation doses $(<0.2 \mathrm{dpa})$ [75]. However, at larger radiation doses, the deformed microstructure changes drastically and is dominated by the formation of dislocation channels, associated with the $\langle 111\rangle\{110\}-$ and $<111>\{112\}$-type slip systems in bcc crystals $[26,75,96,102]$. These dislocation channels are

\footnotetext{
${ }^{2}$ Significant parts of this Chapter have appeared in Ref. [91].
} 
typically depleted of irradiation-induced defects and dislocation debris [103,104], though they might not be entirely free of defects $[75,105]$. The channels are generally found to be oriented along the direction of maximum resolved shear stress in the material, e.g., at $45^{\circ}$ to the loading axis for uniaxial tension [75]. The channels are 50-200 nm wide [26,75,106,107] and the spacing between channels is $\sim 1.5-5 \mu \mathrm{m}$ [75]. It has been found that the channels are formed more densely near the fracture surfaces as compared to the uniformly strained bulk; the average spacing between channels differs by an order of magnitude in these regions [75].

The mechanical behavior of these materials is governed primarily by the dislocationdefect interactions. Irradiation leads to an increase in yield strength of the materials. This is due to the presence of irradiation-induced point defect clusters and dislocation loops, posing as added obstacles in the path of glide dislocations that carry inelastic deformation. More importantly, there is a severe decrease in ductility and engineering strain to failure in these irradiated materials. This observed behavior has been correlated to the formation of defect-depleted dislocation channels mentioned above. It has been suggested [102] that pinning of dislocation sources at (stronger) irradiation-induced defects leads to stress concentration and restricted flow in the material. When the dislocations are eventually unpinned, higher resolved shear stresses allow them to sweep through and annihilate the barriers, hence creating defect-depleted channels [108]. Subsequent glide of dislocations through these 'cleared' channels becomes easier, leading to flow softening and localization of inelastic deformation along the channels [101], finally resulting in failure at relatively lower bulk strains [109].

Physical mechanisms involved in the annihilation of defects by mobile glide dislocations are still a matter of ongoing research. In situ transmission electron microscopy (TEM) and molecular dynamics (MD) simulations are primarily used for this purpose. It has been found that screw dislocations are more effective at annihilating irradiation-induced defects and have higher mobility within the channels as compared to edge dislocations in fcc metals [104,110]. It is noted that majority of the defect annihilation studies have been performed for fcc metals and alloys. Studies of the interaction of dislocations with defect clusters in fcc metals (primarily vacancy type stacking fault tetrahedra, SFT) show that the SFTs can either be sheared by or absorbed into the gliding dislocations [111-115]. The SFTs can also be partially unfaulted on interaction with screw dislocations which spontaneous cross-slip on the face of the SFTs [116]. Interstitial type Frank loops interacting with screw dislocations have been found to be transformed into helical turns, which pin the dislocation lines [117,118]. When the dislocations are eventually unpinned at higher stresses, they carry the helical turns along with them, leading to progressive clearing of dislocation channels $[117,118]$. Similar phenomena have also been suggested for the interaction of SFTs with screw dislocations [116].

In bcc metals, plastic deformation is non-trivial due to the inherent non-planar nature of the $1 / 2\langle 111\rangle$ screw dislocation cores, manifesting in the form of non-associative plastic flow, 
tension-compression asymmetry, and temperature-dependent screw dislocation mobility [119122]. Consequently, the study of defect (primarily interstitial loop) annihilation mechanisms in bec structures is much more difficult, and is also material-dependent. Experimental observations of post-deformed samples of irradiated Fe-9Cr alloy [123] suggest that removal of irradiation induced- $\langle 100\rangle$ loops occurs by continued interaction with $1 / 2\langle 111\rangle$ screw dislocations based on the reaction: $[100]+1 / 2[\overline{1} 11]=1 / 2[111]$. Subsequent transformation of the resultant loops into screw dislocations with the same Burgers vector leads to a microstructure typical of the unirradiated deformed specimens. MD simulations of temperature-dependent reactions between $1 / 2\langle 111\rangle$ screw dislocations and $\langle 100\rangle$ interstitial loops in bcc iron [124] have suggested that the absorption of loops by screw dislocations results in the formation of glissile helical turns at elevated temperatures. This could lead to potential reactions of the gliding helical turns with other radiation induced-defects, resulting in their growth or shrinkage, ultimately contributing to dislocation channel formation. MD studies of edge dislocations interacting with self-interstitial atom (SIA) loops have also suggested other potential defect annihilation mechanisms via loop absorption into the dislocations [125,126]. A recent dislocation dynamics (DD) study of irradiated bcc-Fe [44] suggests that transition to localized deformation occurs at a critical density of the irradiation-induced defects (prismatic loops), below which deformation is mostly homogeneous. It was suggested [44] that flow localization along dislocation channels is a twostep process: initial coarsening of the prismatic loops on their interaction with glide dislocations, followed by 'transformation' of these coarsened prismatic loops into structures that are indistinguishable from network dislocations (in unirradiated materials). As a result, flow resistance along these regions is greatly reduced due to the transformation of prismatic loops to the 'apparent' network dislocations, allowing inelastic deformation to localize.

As can be seen, modeling the localization phenomena in irradiated materials is rather complicated due to the nature of dislocation-defect interactions involved; it has been mostly limited to MD (mentioned above) and DD studies [22,44,117,118,127-129]. There is also an issue associated with the inherent length scale ( hundred $\mathrm{nm}$ ) of the problem. On one hand, coarse-grained calculations need to capture the underlying physics of phenomena occurring at lower length scales; on the other hand, lower scale atomistic studies of such large polycrystalline ensembles over the time frame of a few hundred seconds are impractical. Nonetheless, MD simulations are vital in understanding the defect evolution and individual physical mechanisms at the atomic scale and extracting information for higher scale simulations. Although providing an effective method to model this phenomenon at intermediate length scales, DD simulations are limited in applicability to very small sample sizes and are therefore inadequate for predicting the overall mechanical properties of the bulk. A mechanism-based mesoscale constitutive crystal plasticity continuum framework with adequate phenomenological treatment of the underlying 
deformation processes (including a suitable description of the defect annihilation mechanism, yet to be fully resolved) at lower scales can address the aforementioned scale issues. Moreover, it can be used to study the effect of factors such as crystallographic orientation, grain size, etc. on the localization behavior.

We developed a defect density-based crystal plasticity framework and used it to simulate the mechanical behavior of irradiated bcc materials in Chapter 2. Evolution laws for dislocations and interstitial loops (produced due to radiation in bcc materials) were tied to the inelastic deformation behavior. In addition, rate theory calculations were used to model the evolution of irradiation-induced point defects. However, the constitutive framework did not attempt to model the localization behavior observed in irradiated metals loaded in tension. In this Chapter, a phenomenological model is developed to account for the observed localization due to interaction of irradiation-induced defects with glide dislocations. The framework is used to model the mechanical response and deformed microstructure of an irradiated ferritic/martensitic steel loaded in tension.

\subsection{Model description}

The continuum crystal plasticity model accounts for the climb-enhanced glide of mobile dislocations trapped in the field of immobile dislocations and irradiation-induced interstitial loops, at the level of slip systems. Dislocation densities, both mobile $\left(\rho_{M}^{\alpha}\right)$ and immobile $\left(\rho_{I}^{\alpha}\right)$, number densities of the sessile interstitial loops $\left(N_{i}^{\alpha}\right)$, and interstitial loop sizes $\left(d_{i}^{\alpha}\right)$ are used as ISVs in the model, the evolution of which affect the hardening behavior of slip system $\alpha$. It is assumed that all the interstitial loops are planar and circular in shape. In addition, point defects, i.e., interstitials $\left(c_{i}\right)$ and vacancies $\left(c_{v}\right)$ (generated during irradiation and present thereafter) are tracked using rate theory calculations. As described in Chapter 2, physically-consistent models are used for the evolution of defect densities.

In this Chapter, we develop a phenomenological model for reduction in density of the interstitial loops, to account for the observed flow softening behavior.

\subsubsection{Defect annihilation model}

Using insights from MD simulations for fcc Ni, Rodney et al. [130] developed a micromechanical model that asserts 'absorption' by glide dislocations within a critical capture radius of irradiation-induced defects. However, they assumed that the defects would be absorbed uniformly, without any consideration for preferential slip on favorably oriented planes. A critical capture radius for defect absorption has also been employed in DD simulations of fcc metals by Ghoniem et al. [43,128]. Using this information, Krishna et al. [13] recently developed a constitutive model for defect annihilation in fcc metals. Their model considered dependence of 
the defect annihilation rate on both the defect density and dislocation density, as compared to Rodney et al. [130] where absorption rate is dependent only on the defect density. Further, Krishna et al. [13] used a crystal plasticity framework and also accounted for dependence of the annihilation rate on the crystallographic shearing rate.

Before describing our defect annihilation model, it is again emphasized that the mechanisms of defect annihilation in bcc metals are less well understood and quantified than those in fcc metals (cf. Section 3.2). Assuming that a similar concept of critical capture radius would hold for bcc metals, we have formulated a phenomenological model for the annihilation rate with dependence on both mobile dislocation density, $\rho_{M}^{\alpha}$, and areal loop density, $N_{i}^{\alpha} d_{i}^{\alpha}$. Accordingly, the depletion rate of areal loop density on any slip system $\alpha$ is given as

$$
\dot{N}_{i, \text { ann }}^{\alpha} d_{i}^{\alpha}=-\frac{R_{a n n}}{b}\left(N_{i}^{\alpha} d_{i}^{\alpha}\right)^{c}\left(\rho_{M}^{\alpha}\right)^{1-c}\left|\dot{\gamma}^{\alpha}\right|
$$

where $R_{a n n}$ is the capture radius of annihilation and $c$ is the exponent of the areal loop density such that the product of areal loop density term and mobile dislocation density term on the right hand side of Eq. (3.1) has the units of dislocation/areal loop density. This leads us to the annihilation rate of interstitial loop number density as

$$
\dot{N}_{i, a n n}^{\alpha}=-\frac{1}{d_{i}^{\alpha}} \frac{R_{a n n}}{b}\left(N_{i}^{\alpha} d_{i}^{\alpha}\right)^{c}\left(\rho_{M}^{\alpha}\right)^{1-c}\left|\dot{\gamma}^{\alpha}\right|
$$

Rodney et al. [130] also suggested that the capture radius would increase with increasing loop sizes. Accordingly, the capture radius, $R_{a n n}$, is varied as a function of the current loop size, $d_{i}^{\alpha}$, in this framework, and the areal loop density exponent, $c$, is also varied to study their effect on the localization behavior. Both these parameters are also used for calibrating simulation results to experimental stress-strain data for irradiated specimens.

Recent dislocation dynamics studies of irradiated Fe [44] have suggested that reduction in flow stress may simply be due to transformation of these loops into ordinary network dislocations (without any annihilation/absorption of the loops), thus reducing their resistance to dislocation glide and promoting localization. While this observation may be true, this does not rule out the 'absorption' of irradiation-induced defects by mobile dislocations as observed by others (cf. Section 3.2). With more physical insights, appropriate modification to the phenomenological rule given in Eq. (3.2) may be made to account for this mechanism in the future. Nevertheless, our constitutive model in its present form accounts for the reduction in loop density, and consequent reduction in flow stress, enabling us to study localization as a function of other mesoscopic factors, such as crystallographic orientations, loop density, etc.

The updated rate of evolution of interstitial loop density (modified from Eq. (2.27)) is thus given as 


$$
\dot{N}_{i}^{\alpha}=\dot{N}_{i, c \lim b}^{\alpha}+\dot{N}_{i, a n n}^{\alpha}
$$

Note that mobile dislocations also get (statistically) trapped at the interstitial loop barriers and 'transform' to immobile dislocations. This is modeled using the last term in Equation (2.25).

\subsection{Model implementation}

\subsubsection{Model parameters}

The constitutive model was used to simulate the tensile behavior of Mod 9Cr-1Mo steel. This steel has good radiation resistance and high temperature properties. Ferrite and martensite constitute the main phases of Mod 9Cr-1Mo steel, which derives its strength from the lath martensite microstructure. Moreover, the crystal structure of the subsequently tempered martensite phase is closer to body-centered cubic than body-centered tetragonal [77]. Thus, we have assumed the material to have a uniform bcc structure with 48 slip systems. The majority of the parameters in this model require estimation from physical constants. For Mod 9Cr-1Mo steel, however, not much physical data are available apart from the elastic constants. Since, it has primarily ferritic and martensitic phases, most of the parameters are estimated from available data for bcc-iron. The rationale behind selecting specific values has already been explained in detail in Section 2.4.1 and the corresponding values given in Table 1 in Chapter 2.

Experimental studies of the microstructure of Mod 9Cr-1Mo steel have estimated a high density of dislocations of the order $10^{7}-10^{8} \mathrm{~mm}^{-2}$ [88,89]. Based on this, the initial density of

mobile dislocations is assumed to be $\rho_{M}^{0}=2 \times 10^{7} \mathrm{~mm}^{-2}$ and that of $\rho_{I}^{0}=3 \times 10^{7} \mathrm{~mm}^{-2}$ is assumed for immobile dislocations on all 48 slip systems in our simulations.

In this Chapter, a parametric study of the cross-slip model and its effects on localization behavior has been performed. An analytical solution for the critical activation stress for crossslip, $\tau^{*}$, is given by Hirth and Lothe [66]. The value of $\tau^{*}$ depends on the average dislocation line length. Given an average dislocation density of $1 \times 10^{7}-5 \times 10^{7} \mathrm{~mm}^{-2}$ per slip system, the average dislocation line length $\approx 100-200 \mathrm{~b}$. Corresponding to this dislocation line length, $\tau^{*}$ was estimated to be $\approx 0.004 G$ from Rhee et al. [67]. The same value was used in all our simulations. The activation volume for cross-slip was assumed to be $V_{a}=20 b^{3}$. The value of the material constant, $k_{c s}$, was varied to study its effect on the microstructure evolution.

\subsubsection{Loading and boundary conditions}

The 3D constitutive model was implemented in the finite element program ABAQUS [86] using the User MATerial (UMAT) subroutine. Simulations were performed using 
generalized plane strain elements along with the 3D crystallography, effectively treating a network of cubic grains (square cross-section in the plane) as columnar with random orientation distribution. Finite elements (FEs) with linear interpolation and reduced integration were used.

Quasi-static tension tests were simulated on the polycrystalline ensembles. A schematic of the loading and boundary conditions is shown in Fig. 10. The bottom edge of the 2D simulation box was constrained along the loading direction, and the center of the bottom edge was constrained in all degrees of freedom to prevent rigid body rotation. The side edges of the simulation box were traction-free. Note that traction-free surfaces are valid boundary conditions only for loading samples to relatively small strains ( 4-5\% strains in the present case). Displacement-controlled tensile loading was applied on the top edge at a prescribed strain-rate of $1 \times 10^{-4} \mathrm{~s}^{-1}$ in all simulations.

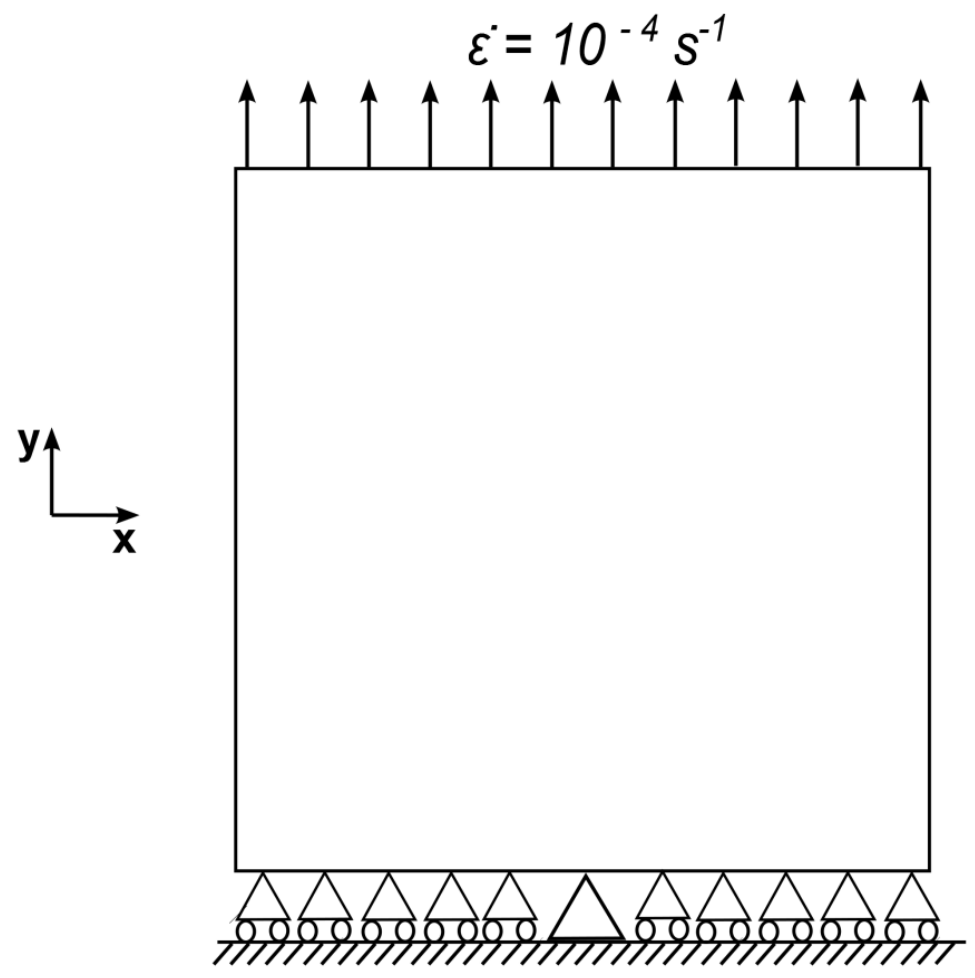

Figure 10. Schematic of loading and boundary conditions.

\subsection{Results and discussion}

Post-irradiation tension tests were performed on 25 square-shaped, randomly oriented, 5 $\mu \mathrm{m}$-sized grains, unless mentioned otherwise. Post-irradiation tension tests are those for which the material is loaded in a normal (no radiation) environment following irradiation to a certain dpa damage level. The presence of prior radiation damage leads to dislocation channel formation 
and inelastic strain localization during these tensile tests. Note that in-reactor tension test (concurrent radiation and deformation with little or no prior radiation history) studies on bcc-Fe and Fe-Cr alloys by Singh et al. [131] found that deformation is homogeneous and dislocation channels are not formed. However, in-reactor tension test studies on $\mathrm{Cu}$ and $\mathrm{CuCrZr}$ alloys [132] did find the formation of dislocation channels, though yield drop and plastic instability was not observed in the associated mechanical behavior. These experiments suggest that channel formation and plastic instability is strain rate- and radiation dose-dependent.

The following sections will first consider the simulated mechanical response, and then describe the simulated deformed microstructure in terms of the evolution of effective inelastic strain, mobile dislocation density, and interstitial loop density.

\subsubsection{Mechanical behavior}

Certain model parameters were calibrated to match the experimental engineering stressstrain data for Mod 9Cr-1Mo steel [2]. The model was first fit to the virgin specimen data using the parameter values given in Table 1 . Values of $k_{c s}=2.0 \times 10^{4}, R_{a n n} / d_{i}^{\alpha}=3.0$ and $c=0.85$ for the cross-slip and flow softening models were then used to match the model results to the irradiated specimen data. Figure 11 shows the simulated stress-strain curves and the corresponding experimental data for various loading and irradiation histories. A reasonable fit is obtained for all cases, to 3-4\% engineering strain for irradiated specimens, and 5-6\% engineering strain for virgin specimens. Note the change in the stress-strain response of the irradiated specimens as a result of the additional defect annihilation model (Eq. (3.2)) from the initial stress-strain curves shown previously in Fig. 2. For irradiated specimens, the initial localization behavior is dominated primarily by material softening instabilities due to formation of dislocation channels. At higher strains, geometric softening instabilities are expected to set in, which the current simulation meshes and boundary conditions do not address; only material instabilities are captured. For virgin specimens, no material softening instabilities are present for the simulated mechanical response. The softening observed in the experimental stress-strain curves of the virgin specimens is dominated by geometric instability due to diffuse necking.

For the post-irradiation tensile tests simulated here, diffusion gradients were neglected in the point defect rate equations (Eqs. (2.9), (2.10)). This is a valid assumption given the fact that there is no concurrent irradiation and the overall point defect concentrations are low in the material. However, for the case of in-reactor tension tests (cf. [131,132]), with concurrent irradiation, the diffusion gradients cannot be neglected, and will play a role in the evolution of the deformed microstructure. Future work will focus on modeling these experiments.

The objective of the present modeling is to determine the localization patterns and how

they relate in terms of spatial frequency and orientation to the larger scale polycrystalline grain 
structure. The simulation results presented in Fig. 11 were carried out using finite elements with a mesh size of $200 \mathrm{~nm}$. This mesh size is selected as the minimum size which serves as a compromise between a converged polycrystal stress-strain response and the lower limit of the continuum field approximation of continuously distributed dislocations (cf. [133]). The current dislocation mechanism-based constitutive framework admits interpretation of the dislocation spacing in channels and allows for estimation of continued channel refinement and enhanced strain localization without having to explicitly resolve the fields below the minimum mesh size of $200 \mathrm{~nm}$. Even if the measured channel width is below this scale, the net shear strain is comparable at the mesoscale (e.g., grain scale) in both cases. At a resolution of $200 \mathrm{~nm}$ element size, each finite element would contain $\sim 2-4$ dislocations per slip system (for a given dislocation density $\sim 5 \times 10^{7}-10^{8} \mathrm{~mm}^{-2}$ ). A finer mesh would effectively have less than one dislocation per element. This provides a lower limit on the element size for purposes of a statistical ensemble representation.

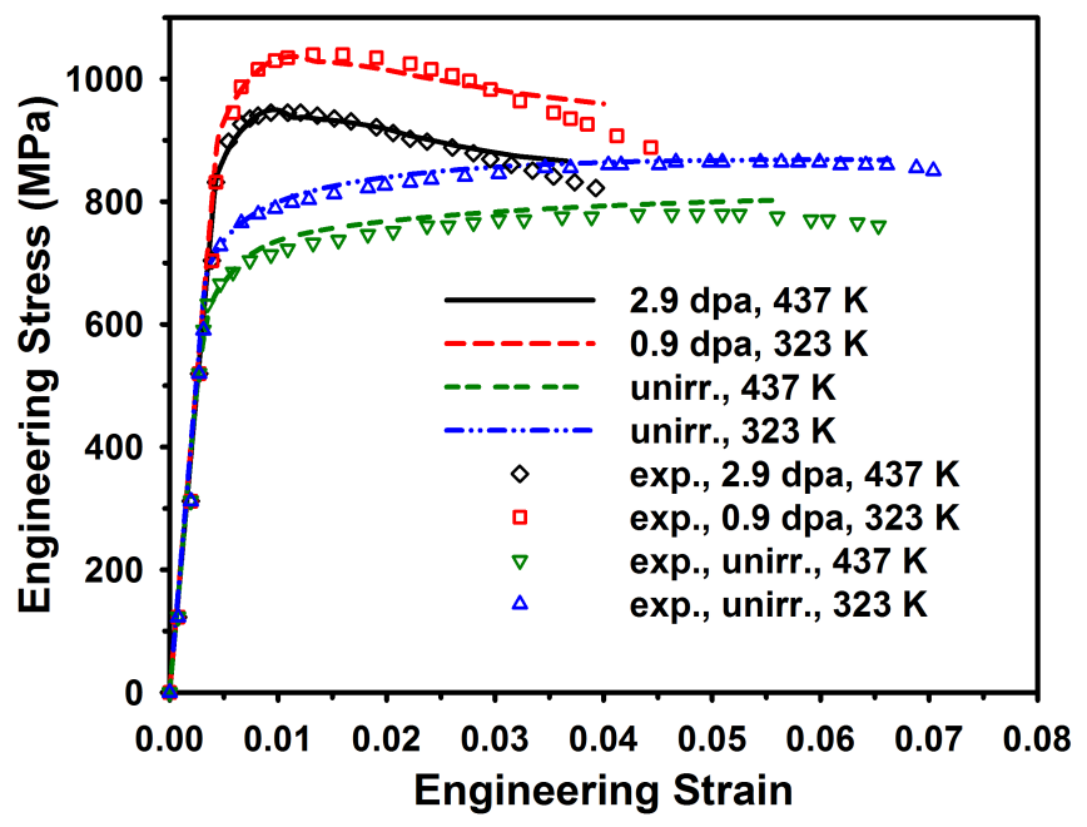

Figure 11. Comparison of simulated stress-strain curves (solid and dotted lines) calibrated to experimental data (open symbols). Experimental data from Maloy et al. [2].

Effect of element size on the inelastic part of the stress-strain response was analyzed by coarser mesh simulations for the case of 2.9 dpa radiation damage at $437 \mathrm{~K}$ (Fig. 12). It was found that the mechanical response of the material depends somewhat on the uniform element size. Using the same set of parameters, a reasonable fit to the experimental stress-strain curve was obtained for all element sizes, at least until geometric instabilities set in later on. However, 
note that the drop in stress due to localization instabilities is much larger when using a coarse mesh. This is because localized deformation takes place over a relatively larger volume of the coarse mesh, thus contributing to the (volume averaged) stress response more significantly. Convergence was achieved in the peak stress and initial localization regime for element sizes approaching $200 \mathrm{~nm}$. An element size of $200 \mathrm{~nm}$ will be used in the following simulations, unless specified otherwise.

Finally, these simulations were performed using $5 \mu \mathrm{m}$-sized grains. This was done to homogenize the effect of random initial crystallographic orientations on the deformation behavior, and still use a relatively small simulation cell size (with a very fine mesh). We have verified using larger grain simulations that grain size effects on the yield strength and hardening behavior are not noticeable. In the present form of model there are no scale effects to reflect this. However, the element size and the grain size affect localization behavior in terms of the dislocation channel width and the channel spacing, respectively. These effects will be discussed in detail in Section 3.5.6.

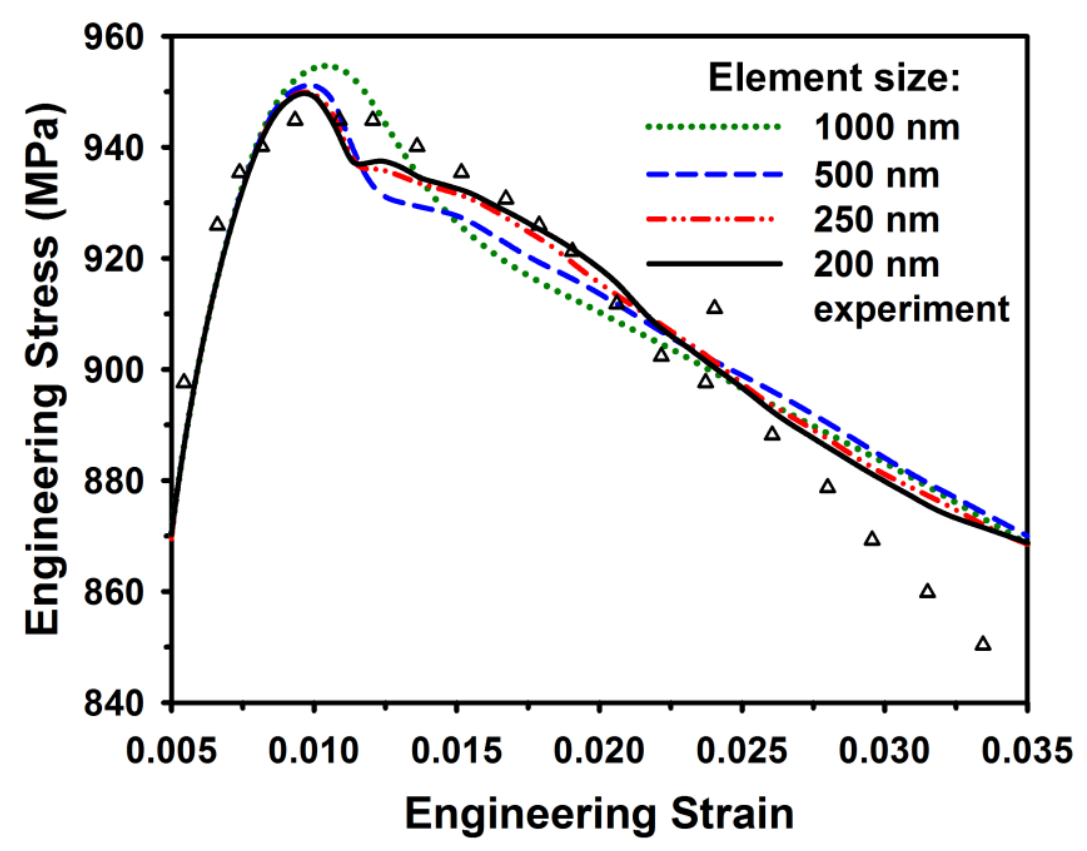

Figure 12. Effect of element size on the inelastic part of the stress-strain curves for the material loaded at $437 \mathrm{~K}$ after irradiation to $2.9 \mathrm{dpa}$. Open triangles indicate the corresponding experimental data. 


\subsubsection{Microstructure evolution}

\subsubsection{Evolution of inelastic strain}

The microstructure of irradiated materials loaded in tension is dominated by the formation of defect-depleted dislocation channels. This results in highly localized deformation along narrow shear bands. To visualize this phenomenon, the distribution of effective inelastic strain was plotted after tensile deformation to $2 \%$ engineering strain in Fig. 13 (a), and subsequently $4 \%$ engineering strain in Fig. 13 (b). The sample was tested at $437 \mathrm{~K}$, following irradiation to $2.9 \mathrm{dpa}$. Values of $k_{c s}=2.0 \times 10^{4}, R_{a n n} / d_{i}^{\alpha}=3.0$ and $c=0.8$ were used for the cross-slip and defect annihilation parameters, respectively. Regions A, B and C marked in Fig. 13 (b) indicate the microstructure evolution during the stages of dislocation channel formation. These regions will be discussed later in the context of defect evolution.

(a)

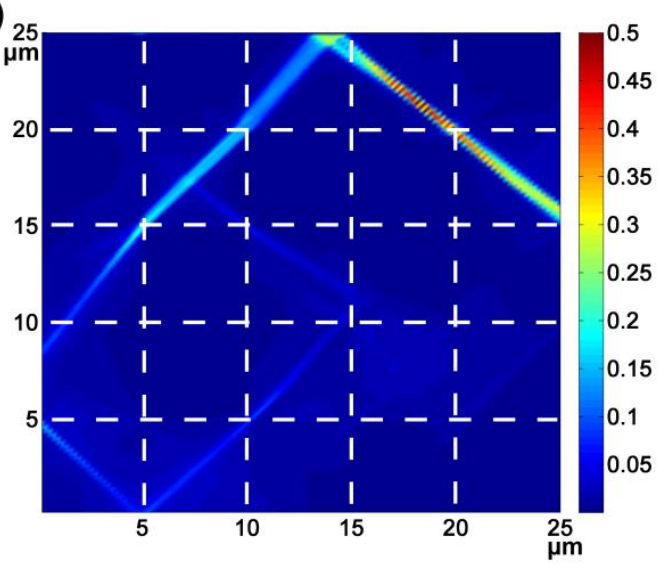

(b)

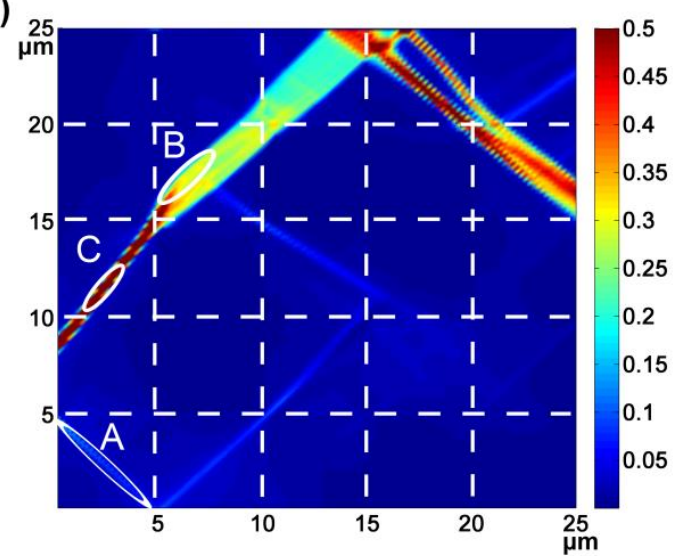

Figure 13. Distribution of effective inelastic strain for material loaded in tension at $437 \mathrm{~K}$ after (a) $2 \%$, and (b) $4 \%$ applied engineering strain. The material was irradiated to 2.9 dpa prior to tensile loading. Dotted lines overlaid indicate grain interfaces. See text for description of regions $\mathrm{A}, \mathrm{B}$, and $\mathrm{C}$.

As shown in Fig. 13, intense shear bands, with inelastic strains as high as $\approx 0.7$, are formed when the average engineering strain in the bulk is much lower $(\approx 0.02-0.03)$. The shear bands (due to channels) are about 200-500 $\mathrm{nm}$ and the average spacing between the bands is of the order of $10 \mu \mathrm{m}$. The dislocation channel width is over-predicted by a factor of $2-4$ as compared to the experimental results of Zinkle and Singh [75]. Also, the inelastic strain values in our simulations are lower than the experimentally measured shear strains ( 1.0-5.0) [96,134]. As mentioned in the last section, the $200 \mathrm{~nm}$ mesh size in these simulations facilitated applicability of the mechanism based continuum approach at or above the scale of mean dislocation spacing in 
the channels, which allows us to consider a polycrystalline ensemble with a number of grains, addressing issues such as the effects of microstructure on channel formation and spacing. Presently, discrete dislocation mechanics models, also a continuum theory that introduces dislocation lines within a lattice, are not sufficiently advanced to consider the large scale of polycrystals (such approaches are typically used to model dislocation substructure formation), to address dislocation interactions with grain boundaries, or to consider in a robust way the problem of numerous dislocation interactions with irradiation induced defects, including effects of local lattice rotations within shear bands (cf. [117,128,129,135]). Since the net shear strain at the polycrystal level due to localized plasticity is essentially the same regardless of channel width, the product of the inelastic shear strain within the channel and the channel width should be more or less invariant; in the present case of a $200 \mathrm{~nm}$ minimum channel width, the computed localized plastic shear strains should be multiplied by a factor of roughly 4 to project the inelastic shear strain levels in the actual channels with $50 \mathrm{~nm}$ width, for example. This is indeed within the window of reported values of localized inelastic strain on the order of 2-3.

Inelastic strain evolution in the microstructure (Figs. 13 (a) and 13 (b)) suggests that multiplication of channels occurs during later stages of deformation; not all channels are formed simultaneously. Further, the channels grow at different rates; those oriented along favorable slip planes grow preferentially. Also, as expected, the inelastic strain in channels increases as the applied engineering strain goes from $2 \%$ to $4 \%$. The widening of channels from Fig. 13 (a) to Fig. 13 (b) might be an artifact of FE simulations given the present assignment of uniform initial defect density. In experiments, channel widening with increasing applied strains is seldom observed in majority of the metals, and it is believed that the channels, once formed during the initial stages, allow all the applied deformation to localize within them. Introduction of a nonuniform distribution of initial defect density, in conjunction with softening, has been shown (cf. [136]) to provide enhanced sharpening of shear bands in alpha-Ti using a crystal plasticity continuum formulation, and may play a similar role in the present case (cf. Section 3.5.7 for the effect of non-uniform initial defects on channel width). However, in an experimental study by Byun et al. [137], evidence of the formation of channels at different points in time was shown for bcc vanadium. They also showed evidence of widening and bifurcation channels, attributing this to multiple cross-slip of screw dislocations within the channels in bcc vanadium. Note that the present model framework considers the (rate-dependent) cross-slip mechanism; widening and bifurcation of channels observed in our simulations are in part due to cross-slip (discussed in detail in Section 3.5.4). From a continuum perspective, it is intuitive that if a 'soft' channel cannot accommodate all the applied deformation, it has to widen. Cross-slip offers a possible mechanism to this end.

It has also been observed experimentally that the channels propagate at $\sim 45^{\circ}$ to the loading direction [75]. The same was verified in our simulations. In Figs. 13(a) and 13(b), the 
vertical direction is the loading axis. Note that the $45^{\circ}$ direction to the loading axis also corresponds to the maximum resolved shear stress plane.

The constitutive framework assumes a homogeneous local material, i.e., neglects gradients of strain or (line, point) defect densities in the material. Generally, heterogeneous localized deformation is associated with gradients (discussed in detail in Section 3.5.8), and has contributions from both material and geometric instabilities, leading to mesh dependence in FE simulations. However, as has been demonstrated, the present framework accounting only for local 'constitutive' material softening due to dislocation channel formation is able to qualitatively capture both the microstructure evolution and mechanical behavior of irradiated materials. Heterogeneous localized deformation, in the present case, is a consequence of spatial correlations of crystallographic orientations of the grains. Dislocation channels form along favorably oriented slip planes, which have achieved higher resolved shear stresses to annihilate the defects. Further, geometric instabilities are not dominant, at least during the early stages of localization $(<4 \%$ engineering strain for the present material). Experiments suggest that these irradiated specimens fail at $\approx 6-8 \%$ engineering strain [2]. At such low strain levels, geometric softening effects, though present during the later stages of localization behavior, might not be significant.

\subsubsection{Substructure evolution}

To understand the underlying physical processes governing dislocation channel formation, it is instructive to observe the defect evolution in the material. Figs. 14 (a) and (b) show the distribution of the mobile dislocation density, $\rho_{M}$, and interstitial loop density, $N_{i}$ (averaged over all slip systems), respectively, corresponding to the effective inelastic strain shown in Fig. 13 (b). Based on the regions A, B and C marked in each of the Figs. 13 (b), 14 (a) and 14 (b), three different stages of microstructure evolution can be identified during dislocation channel formation.

\section{Incipient channel formation}

Region A marks the incipient stages of channel formation, when interstitial loops have just started getting annihilated. The mobile dislocation density is slightly lower than the bulk average, as trapping of mobile dislocations by interstitial loops has rendered them immobile.

\section{Defect depletion}

Once the mobile dislocations are subject to sufficient driving force (resolved shear stress), they start annihilating the interstitial loops. As a result, the interstitial loop density is depleted to lower values than the bulk average in region B. Many of the mobile dislocations are still getting trapped at barriers due to the immobilization process, the rate of which has increased due to continual accumulation of immobile dislocations contributing to statistical trapping of the mobile dislocations. 


\section{Flow localization}

Finally, the flow resistance is lowered due to depletion of interstitial loop density to very low values in region $C$. As a result, easier dislocation glide leads to large inelastic strain in this region. The mobile dislocation density is replenished to relatively high values by dislocation multiplication processes in the defect-depleted channels as a result of enhanced inelastic deformation.

Average values of the mobile dislocation density, interstitial loop density and inelastic strain in the three regions are summarized in Table 2. The interstitial loop density shown in Fig. 14 (b) and reported in Table 2 is averaged over all 48 slip systems. Generally, localization takes place on just a few slip systems, on which majority of the interstitial loops get depleted. Hence, the plotted slip system averaged loop density, also accounting for interstitial loops on slip systems for which localized deformation has not taken place, does not diminish to very low values in the dislocation channels, and differs from the bulk average loop density by a factor of less than 2. In reality, channels are depleted of the majority of the irradiation-induced defects (interstitial loops in the present case), if not entirely cleared [75,105]. Irrespective of this, the stages of microstructure evolution during dislocation channel formation would remain the same.

(a)

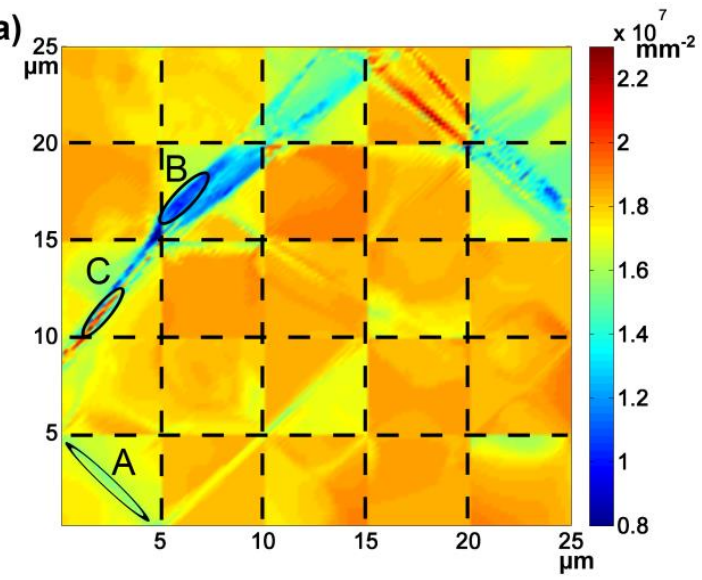

(b)

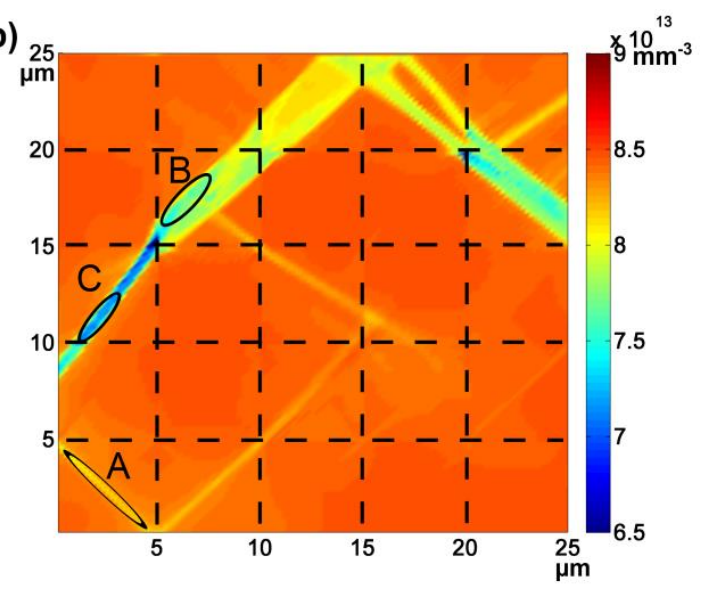

Figure 14. Distribution of (a) mobile dislocation density, and (b) interstitial loop density corresponding to the effective inelastic strain in Fig. 13(b). See text for description of regions $A, B$ and $C$.

Recent TEM studies of the migration of dislocations through a field of irradiationinduced defects by Briceño et al. [138] offer valuable insights regarding the dislocation-defect interactions just prior to localization. The modeled substructure evolution is in agreement with the TEM observations, i.e., mobile dislocations get trapped in a field of defects, thus rendering them immobile, followed by percolation of dislocations through the field as a result of 
annihilation of defects. They also suggested that pumping of mobile dislocations takes place via sources not induced by irradiation, and percolation of the dislocations by defect annihilation only enhances their activation. This is consistent with our constitutive model, i.e., dislocation multiplication takes place irrespective of the irradiation-induced defect field $\left(1^{\text {st }}\right.$ term in Eq. (2.25)), and annihilation of defects only leads to enhanced dislocation migration and larger inelastic strain.

Table 2. Average values of mobile dislocation density, interstitial loop density, and inelastic strain during the different stages of dislocation channel evolution, based on regions marked in Figs. 4(b), 5(a) and 5(b).

\begin{tabular}{lllll}
\hline Region & Stage & $\begin{array}{l}\text { Mobile dislocation } \\
\text { density }\left(\mathrm{mm}^{-2}\right)\end{array}$ & $\begin{array}{l}\text { Interstitial } \\
\text { density }\left(\mathrm{mm}^{-3}\right)\end{array}$ & loop \\
\hline $\mathrm{A}$ & $\begin{array}{l}\text { Incipient channel } \\
\text { formation }\end{array}$ & $1.6 \times 10^{7}$ & $8.0 \times 10^{13}$ & 0.10 \\
$\mathrm{~B}$ & Defect depletion & $1.1 \times 10^{7}$ & $7.7 \times 10^{13}$ & 0.30 \\
$\mathrm{C}$ & Flow localization & $2.1 \times 10^{7}$ & $6.7 \times 10^{13}$ & 0.70 \\
Bulk & --- & $1.8 \times 10^{7}$ & $8.5 \times 10^{13}$ & 0.04 \\
\hline
\end{tabular}

\subsubsection{Effect of radiation dose on localization}

Experimental studies by Sharp [134] and more recently by Jiao et al. [105] show that the inelastic strain in channels (experimentally interpreted in terms of the height of channels) increases with increasing dpa damage (increasing loop size and density). The same was observed in our simulations as the radiation dose was increased from $1 \mathrm{dpa}$ to $2.9 \mathrm{dpa}$. The measured values of maximum inelastic strain in the channels are given in Table 3. All simulations were performed with the same initial microstructures (crystallographic orientation distributions). As seen in Table 3, the maximum inelastic strain in channels increases with increasing dpa dose. In fact, the simulated maximum inelastic strain was found to scale linearly with the radiation dpa dose at both $2 \%$ and $4 \%$ engineering strains (Fig. 15). Note that we have assumed a square root dependence of the interstitial loop density and size on dpa dose (Eqs. (2.30), (2.31)). This suggests that maximum inelastic strain in the channels also has a square root dependence on the interstitial loop density and size.

Note that the linear dependence of maximum inelastic strain in channels as a function of dpa dose emerges from our simulations using the same initial microstructures and the assumption 
of an initially uniform defect distribution. In physical experiments, it is highly unlikely that different samples with different dpa dose histories would have the same initial microstructures; linear dpa dependence has not been reported experimentally. Interestingly, if one were to plot the surface slip band (correlated to dislocation channels in the interior, cf. [139]) heights as a function of the corresponding neutron dose fluxes at the same extension levels reported for irradiated single crystal copper in the table in Ref. [134], it is found that the slip band height does have a near-linear dependence on the neutron dose flux for the limited reported data. However, more systematic experiments are needed to address this issue and verify our model predictions.

Maximum inelastic strain in the channels given in Table 3 depends strongly on the initial crystallographic orientation distributions in the material. For example, using a different initial (random) crystallographic orientation distribution, maximum inelastic strain in the channels is as high as 1.198 at $4 \%$ engineering strain, 2.9 dpa radiation dose and $437 \mathrm{~K}$ (using $k_{c s}=2.0 \times 10^{4}$, $R_{\text {ann }} / d_{i}^{\alpha}=3.0$ and $c=0.8$ ).

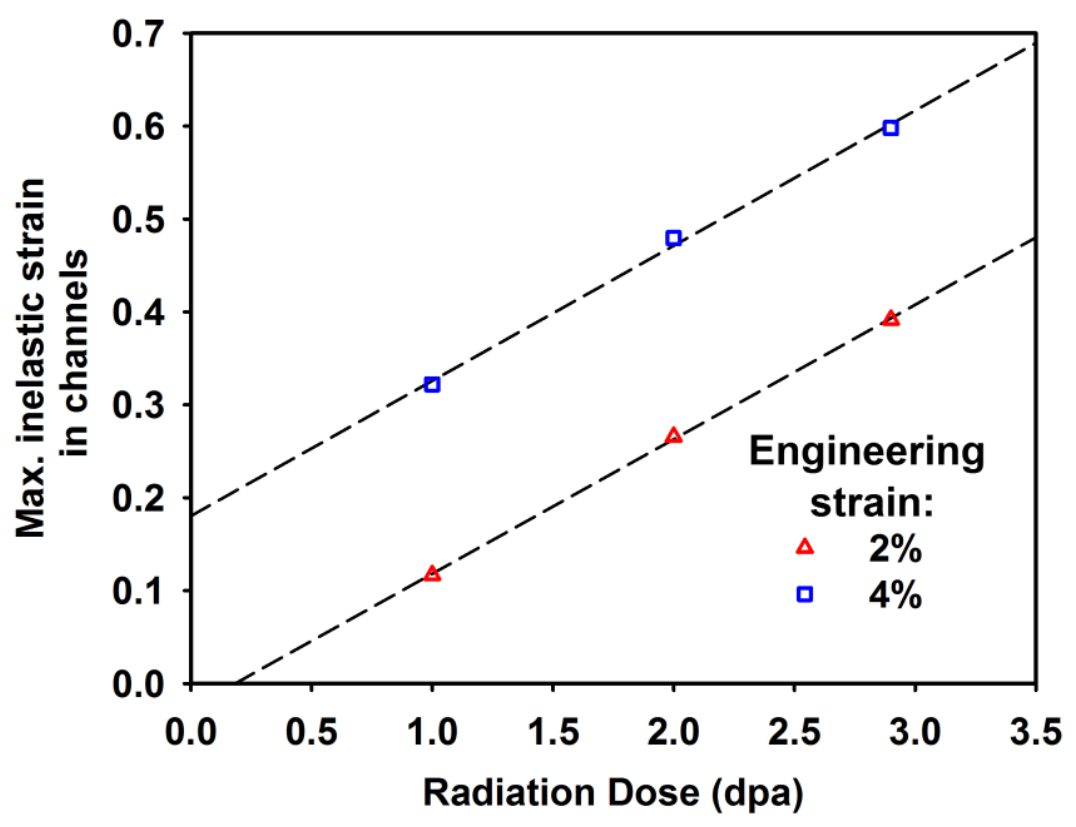

Figure 15. Maximum inelastic strain in dislocation channels as a function of dpa dose after tensile loading to $2 \%$, and $4 \%$ engineering strains at $437 \mathrm{~K}$. 
Table 3. Maximum inelastic strain in channels as a function of radiation dose, engineering strain, $k_{c s}, R_{a n n}$ and $c$. Material was loaded in tension at $437 \mathrm{~K}$. The same initial microstructure was used in all simulations.

\begin{tabular}{|c|c|c|c|c|c|}
\hline $\begin{array}{l}\text { Radiation } \\
\text { dose (dpa) }\end{array}$ & $k_{c s}$ & $R_{a n n} / d_{i}^{\alpha}$ & $c$ & $\begin{array}{l}\text { Engineering } \\
\text { strain }\end{array}$ & $\begin{array}{l}\text { Maximum inelastic } \\
\text { strain in channels }\end{array}$ \\
\hline \multirow[b]{2}{*}{1} & \multirow[b]{2}{*}{$2.0 \times 10^{4}$} & \multirow[b]{2}{*}{3.0} & \multirow[b]{2}{*}{0.8} & $2 \%$ & 0.117 \\
\hline & & & & $4 \%$ & 0.303 \\
\hline \multirow{2}{*}{2} & \multirow{2}{*}{$2.0 \times 10^{4}$} & \multirow{2}{*}{3.0} & \multirow{2}{*}{0.8} & $2 \%$ & 0.266 \\
\hline & & & & $4 \%$ & 0.479 \\
\hline \multirow{2}{*}{2.9} & \multirow{2}{*}{$2.0 \times 10^{4}$} & \multirow{2}{*}{3.0} & \multirow{2}{*}{0.8} & $2 \%$ & 0.392 \\
\hline & & & & $4 \%$ & 0.598 \\
\hline \multirow{2}{*}{2.9} & \multirow{2}{*}{$2.0 \times 10^{6}$} & \multirow[b]{2}{*}{3.0} & \multirow[b]{2}{*}{0.8} & $2 \%$ & 0.359 \\
\hline & & & & $4 \%$ & 0.446 \\
\hline \multirow{2}{*}{2.9} & \multirow{2}{*}{$2.0 \times 10^{8}$} & \multirow{2}{*}{3.0} & \multirow{2}{*}{0.8} & $2 \%$ & 0.252 \\
\hline & & & & $4 \%$ & 0.345 \\
\hline \multirow[b]{2}{*}{2.9} & \multirow{2}{*}{$2.0 \times 10^{4}$} & \multirow[b]{2}{*}{2.5} & \multirow[b]{2}{*}{0.8} & $2 \%$ & 0.360 \\
\hline & & & & $4 \%$ & 0.620 \\
\hline \multirow[b]{2}{*}{2.9} & \multirow{2}{*}{$2.0 \times 10^{4}$} & \multirow[b]{2}{*}{3.5} & \multirow[b]{2}{*}{0.6} & $2 \%$ & 0.099 \\
\hline & & & & $4 \%$ & 0.287 \\
\hline \multirow{2}{*}{2.9} & \multirow{2}{*}{$2.0 \times 10^{4}$} & \multirow{2}{*}{3.5} & \multirow{2}{*}{0.8} & $2 \%$ & 0.401 \\
\hline & & & & $4 \%$ & 0.575 \\
\hline
\end{tabular}

\subsubsection{Effect of degree of cross-slip on localization}

The role of cross-slip in the localized deformation of irradiated materials is not yet completely understood. DD simulations by Diaz de la Rubia et al. [22] showed that cross-slip during defect annihilation plays a role in dislocation channel formation. They proposed that double cross-slip of screw dislocations (during their interaction with irradiation-induced defects) 
back to the slip plane from which they initially started contributes to large inelastic strains and hence the localization process. These observations were consistent with TEM studies by Robach et al. [104], which verified the role of cross-slip in the formation and clearing of dislocation channels.

In the present work, we performed a parametric study of the cross-slip model described in Section 2.3.3.3. The estimation procedure for the cross-slip activation stress, $\tau^{*}$, was mentioned earlier. Here, we vary the cross-slip constant, $k_{c s}$, and study the resulting localization behavior. The simulated samples were irradiated to $2.9 \mathrm{dpa}$ and then loaded in tension at $437 \mathrm{~K}$. Figs. 16 (a) and 16 (b) show the effect of increasing $k_{c s}$ on the evolution of effective inelastic strain. The corresponding interstitial loop density evolution is shown in Figs. 16 (c) and 16 (d), respectively. All parameters except $k_{c s}$ remain same in both these simulations. Further, we started out with the same initial crystallographic orientation distribution in both cases. Values of maximum inelastic strains in the channels as a function of $k_{c s}$ are summarized in Table 3.

As the degree of cross-slip $\left(k_{c s}\right)$ is increased from Fig. 16 (a) to Fig. 16 (b), the channels spread over a wider region and have lower localized inelastic strains. Cross-slip offers a way for mobile dislocations within the channels to bypass slip on planes which have higher flow resistance (due to higher interstitial loop density). As a result, mobile dislocations have migrated to planes with lower interstitial loop densities; lesser interstitial loops are annihilated by mobile dislocations in the localized region during the subsequent loop annihilation process. Therefore, increasing cross-slip results in a relative higher loop density within the channels. This is reflected in the (slip system averaged) interstitial loop density plots shown in Figs. 16 (c) and 16 (d). As a result of lesser interstitial loops getting annihilated, the flow resistance is relatively higher with increasing degree of cross-slip, though it is still lower than regions in the immediate vicinity (where no defect annihilation has occurred). However, stress gradients (with the neighboring regions) are now smeared, thus resulting in the formation of wider and more diffuse shear bands.

There are no quantitative studies to compare the simulated effect of the degree of crossslip on localization behavior. However, the current parametric study suggests that cross-slip assists in the formation of wider dislocation channels. Cross-slip is a material dependent phenomenon; the tendency to cross-slip depends on the stacking fault energy, availability of slip planes, etc. In fact, it was observed in a set of experiments performed on irradiated copper and copper-aluminum alloys $[140,141]$ that the low stacking fault energy alloys do not show channel formation. It was observed that for the low stacking fault energy alloys, glide is confined to only a few slip planes [141] as cross-slip to other planes is much more difficult. This precludes any cleared channel formation. Nevertheless, more detailed studies are needed to find a physical basis for choosing the values of $k_{c s}$ and $\tau^{*}$, perhaps from MD simulations. 
(a)

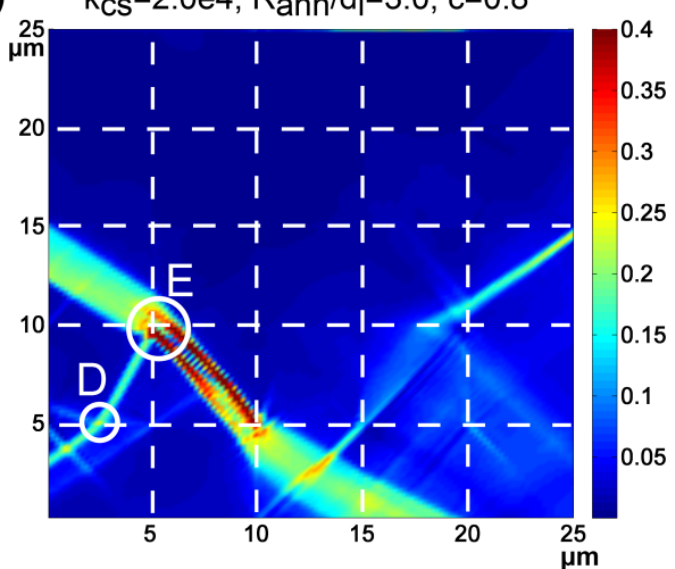

(c)

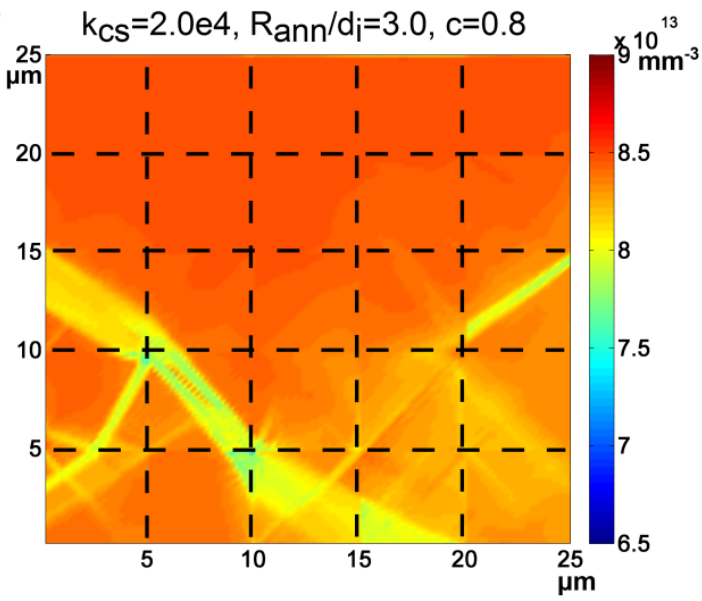

(b) $\mathrm{k}_{\mathrm{cs}}=2.0 \mathrm{e} 8, \mathrm{R}_{\mathrm{ann}} / \mathrm{d}_{\mathrm{i}}=3.0, \mathrm{c}=0.8$

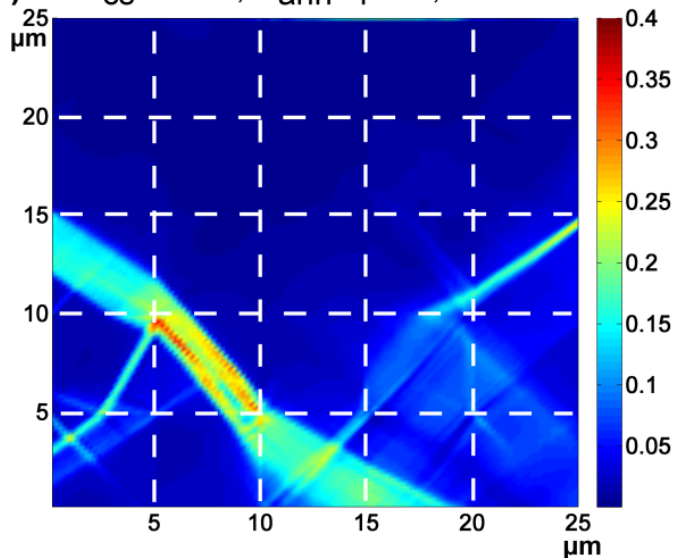

(d)

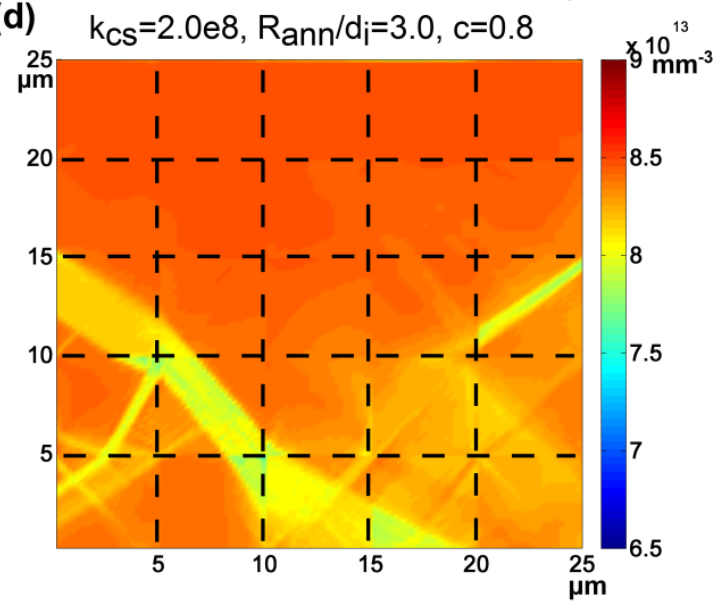

Figure 16. (a), (b): Effect of degree of cross-slip $\left(k_{c s}\right)$ on the distribution of effective inelastic strain for material loaded in tension to $4 \%$ engineering strain at $437 \mathrm{~K}$, after being irradiated to 2.9 dpa damage. (c), (d): Distribution of interstitial loop density corresponding to the effective inelastic strain shown in (a) and (b) respectively. See text for description of regions $D$ and $E$.

On a side note, the structure of grain boundaries is not explicitly accounted for in this model. However, it is known that grain boundaries can act as points of localized stress concentration. This results in a multiaxial stress state and leads to reorientation and bifurcation of dislocation channels. The same was observed in our simulations at the interface between grains where the crystallographic orientations change, for example, in the regions marked D and E in Fig. 16 (a). This is in agreement with experimental observations of reorientation and bifurcation of dislocation channels in bcc materials $[75,137]$. 


\subsubsection{Parametric study of defect annihilation model}

A parametric study of the defect annihilation model and its effect on the localization behavior is presented here. In this phenomenological model (Section 3.3.1), $R_{a n n}$ represents the critical capture radius around a mobile dislocation within which interstitial loops will be annihilated during glide. $R_{a n n}$ was varied as a function of the current interstitial loop size, $d_{i}^{\alpha}$. The exponent of interstitial loop term, $c$, in Eq. (3.2) was also varied.

Figures 17 (a)-(c) show the effective inelastic strain in the material for different combinations of $R_{a n n}$ and $c$. Comparison of corresponding engineering stress-strain curves to the experimental data is shown in Fig. 17 (d). These simulations again represent a material irradiated to 2.9 dpa damage and then loaded in tension to $4 \%$ engineering strain at $437 \mathrm{~K}$. Note that the same initial crystallographic orientation distributions were used as for the cross-slip simulations. Values of maximum inelastic strain in the channels as function of $R_{a n n}$ and $c$ are summarized in Table 3 .

It is found that neither $R_{a n n}$ nor $c$ have a major effect on the channel width. However, the localized inelastic strains and the stress-strain responses are greatly affected. It is observed from Table 3 that as $R_{a n n}$ is increased from $2.5 d_{i}^{\alpha}$ to $3.5 d_{i}^{\alpha}$ (for $c=0.8$ ), the maximum inelastic strain in channels increases monotonically at $2 \%$ engineering strain but decreases monotonically at $4 \%$ engineering strain. This suggests that higher $R_{a n n}$ promotes annihilation of defects initially, so that less number of defects are left in the localized region during subsequent deformation. Consequently, localization is accelerated initially with increasing $R_{a n n}$, but is slowed down later. The parameter $c$ has a more drastic effect on the localization behavior. It is observed that as $c$ is decreased from 0.8 to 0.6 (for $R_{a n n}=3.5 d_{i}^{\alpha}$ ), the localized strains decrease to negligible levels. This implies that the phenomenological rule given in Eq. (3.2) needs to be weighted higher in terms of the current interstitial loop density than the current dislocation density, for localization to occur. 
(a)

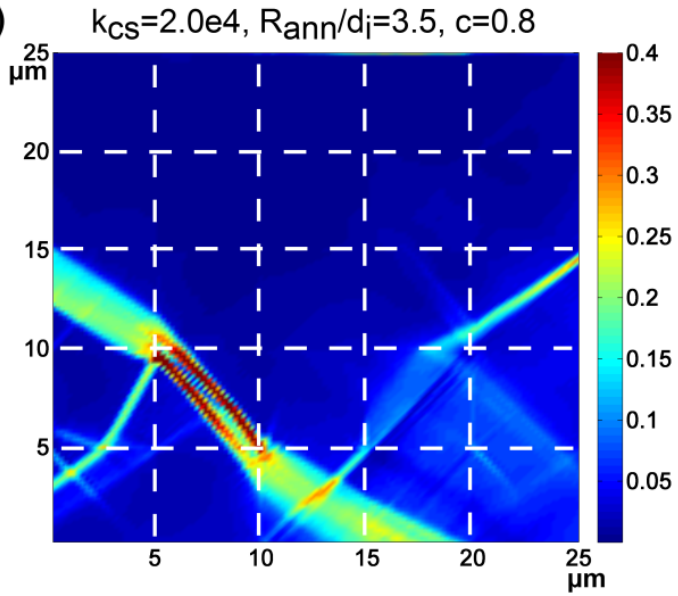

(c)

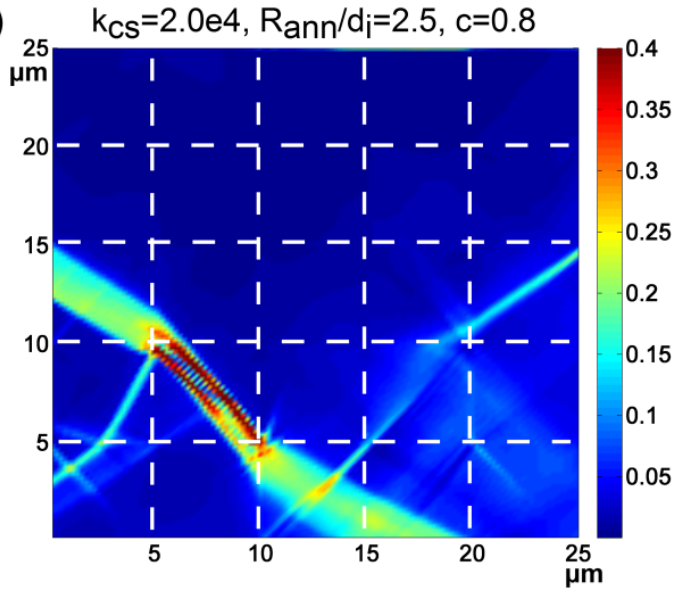

(b)

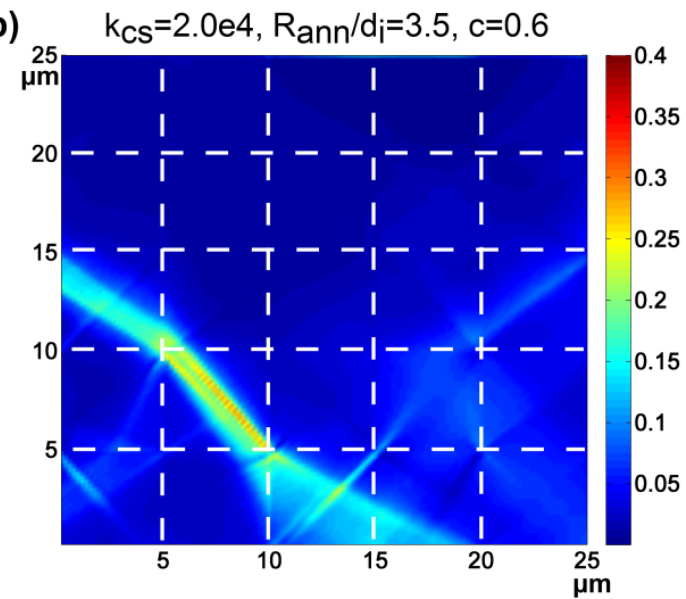

(d)

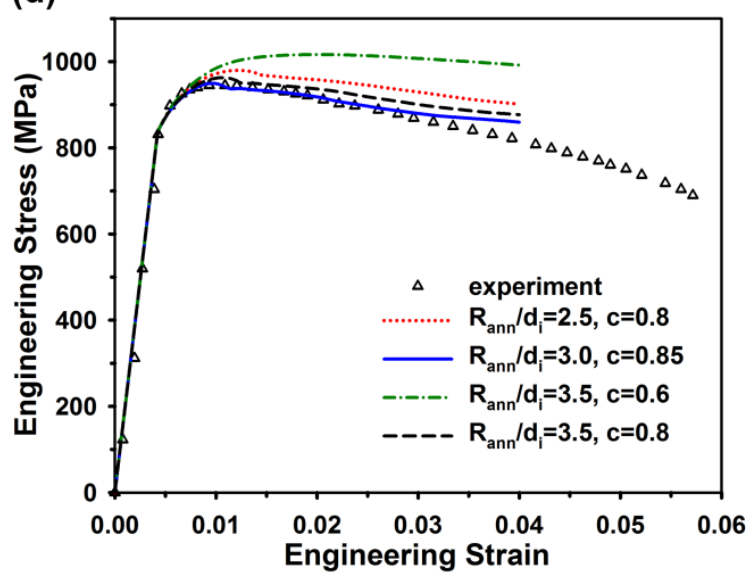

Figure 17. (a-c): Effect of $R_{a n n}$ and $c$ on the distribution of effective inelastic strain in a material loaded in tension to $4 \%$ engineering strain at $437 \mathrm{~K}$, after being irradiation to $2.9 \mathrm{dpa}$. (d): Corresponding effect on the stress-strain curves.

Comparison of the stress-strain curves for different values of the defect annihilation parameters indicates that quite evidently $c$ has a more pronounced effect on the flow stress. The engineering stress decreases by $\approx 150 \mathrm{MPa}$ at $4 \%$ engineering strain as $c$ is increased from 0.6 to 0.8 (for $R_{a n n}=3.5 d_{i}^{\alpha}$ ). However, as $R_{a n n}$ increases from 2.5 to 3.5 (for $c=0.8$ ), the flow stress decreases by $\approx 20 \mathrm{MPa}$. Note that the effect of degree of cross-slip $\left(k_{c s}\right)$ on the stress-strain curves is not as pronounced (difference in engineering stress values $<10 \mathrm{MPa}$ for any given strain, as $k_{c s}$ is increased from $2 \times 10^{4}$ to $2 \times 10^{6}$ ). This is because cross-slip only aids in migration of dislocations to slip planes with lower resolved shear stresses, and does not contribute to the defect annihilation process; mobile dislocations still need sufficient levels of resolved shear stress to annihilate the defects. 
Figure 16 (a) shows the effective inelastic strain plot for $R_{a n n} / d_{i}^{\alpha}=3.0$ and $c=0.8$, other parameters remaining same. Given other parameters, $R_{a n n}=3.0 d_{i}^{\alpha}$ and $c=0.85$ results in the best fit to the experimental stress-strain curve in the present case. Of course, this is not the only exhaustive data set for the model parameters; other combination of parameter values might also yield a good fit to the experimental data. However, the given data set serves the present objective of qualitative parametric analysis of the constitutive model.

\subsubsection{Scale and geometry effects}

Effects of length scale and geometry on the localization behavior were studied by varying the grain size, aspect ratio, and dimensions of the simulation cell. These simulations were performed on larger samples. A relatively coarser mesh (500 nm element size) was used in this case to avoid significantly higher computational times resulting from larger samples with highly resolved meshes; however, by keeping the element size fixed as grain size is varied, the physical scale associated with minimum channel width is established as an a priori assumption of the otherwise local FE analysis. Figure 18 shows the effective inelastic strain in the material for different grain sizes and geometries. All samples were irradiated to 2.9 dpa and then loaded in tension along the vertical axis to $4 \%$ engineering strain at $437 \mathrm{~K}$. Values of $k_{c s}=2.0 \times 10^{4}$, $R_{a n n}=3.0 d_{i}^{\alpha}$ and $c=0.8$ were used in these simulations.

Figures 18 (a) and (b) show the effective inelastic strain in a sample with 100 grains of size $5 \mu \mathrm{m}$, and 25 grains of size $10 \mu \mathrm{m}$, respectively. From simulations, the channel width is not affected by increasing the grain size from $5 \mu \mathrm{m}$ to $10 \mu \mathrm{m}$. However, the corresponding average channel spacing increases from $\approx 10 \mu \mathrm{m}$ to $\approx 20 \mu \mathrm{m}$, indicating that it is dominated by nearest neighbor grain orientation/misorientation effects. For the inelastic strain shown in Fig. 18 (b), the same initial crystallographic orientations were used as for Figs. 13 and 14. However, the grain size is double that of the value used earlier. It is observed that the relative spatial arrangement of dislocation channels in Fig. 18 (b) is the exact same as that observed in Fig. 13 (b). This suggests that dislocation channel orientation and distribution is dependent only on the crystallographic orientations in the material. Further, the spacing between channels is a function of the grain size.

There are two dominant material length scales governing the physics of the localization phenomena. First, the mean free path of mobile dislocations, $l_{d} \propto 1 / \sqrt{\rho_{M}}$, relates to the area swept out on the glide plane, $b l_{d}$ (cf. [65], [16]), by mobile dislocations which annihilate the interstitial loops. Subsequent to defect annihilation, the rate of formation of new mobile dislocations (and hence $l_{d}$ ) governs the width of the channels during the third stage of localization (cf. Section 3.5.2.2, region C in Fig. 14 (a)). Arsenlis et al. [44] suggested a similar 
mechanism governing the characteristic width of dislocation channels. Note that for the present case, $l_{d} \approx 200-225 \mathrm{~nm}$ (given a mobile dislocation density of $2.0-2.5 \times 10^{7} \mathrm{~mm}^{-2}$ along the channels, cf. Table 2). If the FE mesh size is larger than $l_{d}$, then channel formation is regularized by the specified mesh size. In any case, the FE mesh should not reduce below $l_{d}$ as this is considered as the limit of the continuum field approximation. Hence, the channel width is controlled by the greater of $l_{d}$ and the mesh size in these simulations. As discussed earlier, the dislocation mechanism-based constitutive framework admits interpretation of the dislocation spacing in channels and allows for estimation of continued channel refinement and enhanced strain localization without having to explicitly resolve the fields below the minimum mesh size of $200 \mathrm{~nm}$ in the present case.

A second material length scale is the characteristic grain size, which governs the spacing between dislocation channels via heterogeneous triggering of localization. Hence, the channel spacing increases with increasing grain size. This length scale is explicitly modeled in the initial microstructure of the simulated material.

We note that an additional length scale, the diffusion length for interstitials and vacancies, plays a role at very low stress levels and strain rates at elevated temperatures; this may provide additional regularization and coarsening of the channel localization.

Effect of geometry on the channel formation was studied by varying the aspect ratio of the simulation cell. The effective inelastic strain for two such cases is shown in Figs. 18 (c) and 18 (d). A grain size of $5 \mu \mathrm{m}$ was used. Formation of multiple dislocation channels is observed along the longer dimension in both cases. The average channel width and spacing are not affected by changing the aspect ratio of the simulation cell from a square sample to a rectangular (oblate/prolate) sample. This confirms that the localization behavior observed in the present simulations is not an artifact of the geometry and boundary conditions in the simulations; rather, it correlates with the intergranular heterogeneities in the material, notably triggered by grains favorably oriented for slip. 
(a)

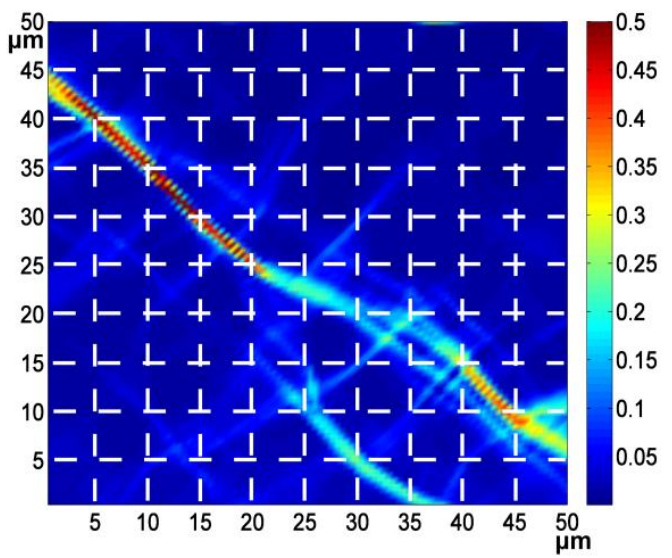

(c)

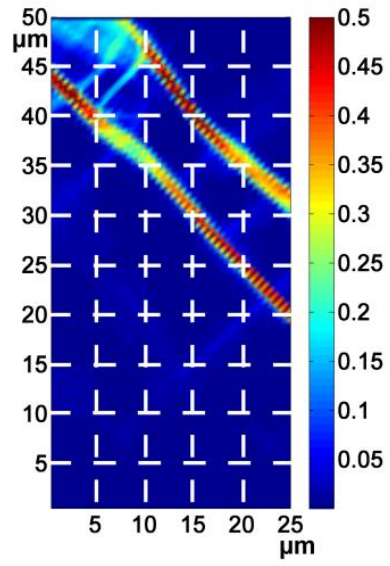

(b)

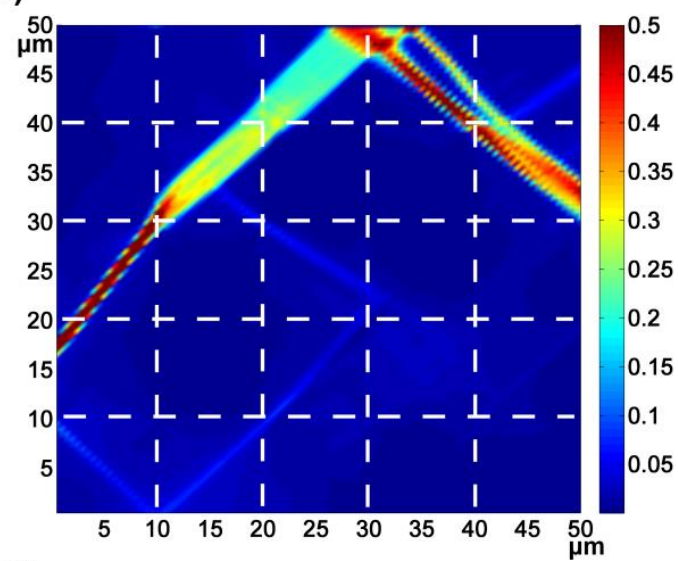

(d)

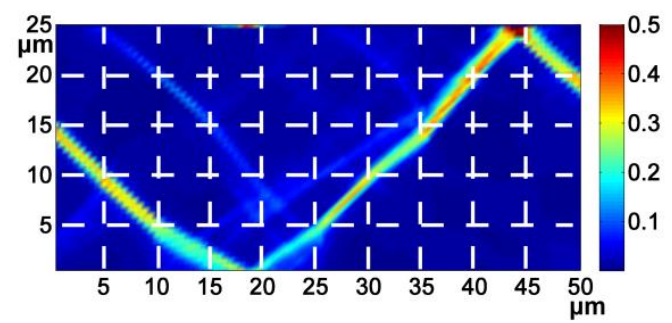

Figure 18. Effect of increasing the grain size from (a) $5 \mu \mathrm{m}$ to (b) $10 \mu \mathrm{m}$ for the same simulation cell size, and (c), (d): effect of simulation cell aspect ratio on the distribution of effective inelastic strain for samples loaded vertically in tension to $4 \%$ engineering strain at $437 \mathrm{~K}$, after being irradiated to $2.9 \mathrm{dpa}$.

\subsubsection{Effect of random defect density on channel width}

Until now, we have assumed that initially the irradiation-induced defects are distributed uniformly in the material. This is an assumption to simplify the model implementation. Physically, it may be expected that the defects are not distributed uniformly. For example, the density of defects may be higher near the irradiated surface. Further, the interstitial loop density may also vary for each slip system, depending on the crystallographic planes on which the interstitial loops are formed. These considerations are beyond the scope of the present continuum framework. However, it has been found earlier [136] that a non-uniform defect density helps to regularize (inhibit) the width of the shear bands and sharpen the degree of localization within them. 
We performed simulations in which each material point in the simulation cell was assigned a random interstitial loop density, with the interstitial loop density having a scatter based on normal (Gaussian) distribution. These simulations were performed with increasing degree of scatter, i.e., standard deviation of the normal distribution was increased from 0.05 to 0.25. The same initial crystallographic orientation was used in all cases. Figures 19 (a)-(c) show the distribution of effective inelastic strain in the material with different degrees of scatter. Figure 19 (d) shows the corresponding inelastic strain profile along a horizontal section of the material at $y=18 \mu \mathrm{m}$, where $y$ represents the vertical direction.

(a)

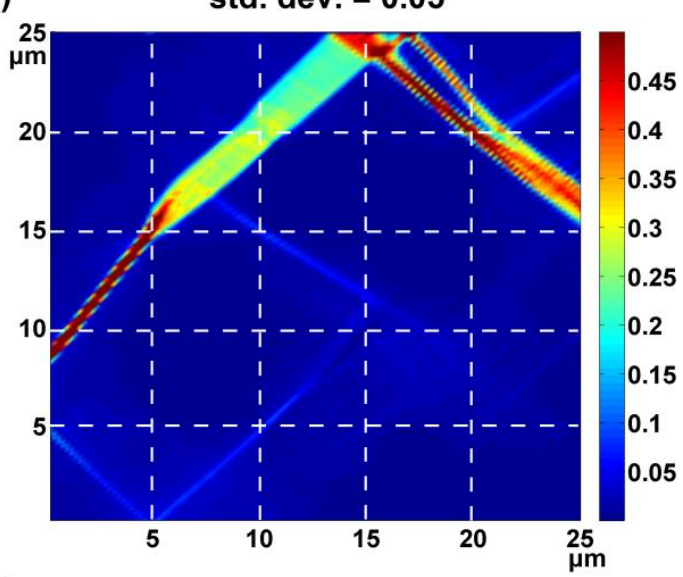

(c)

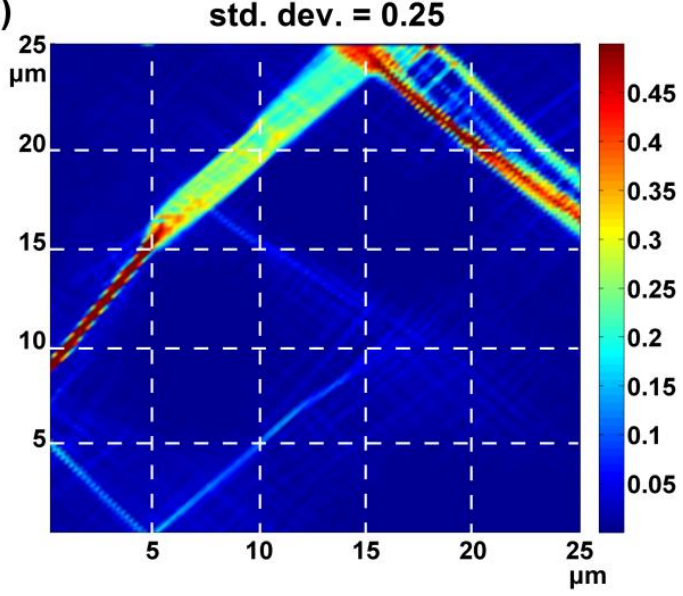

(b)

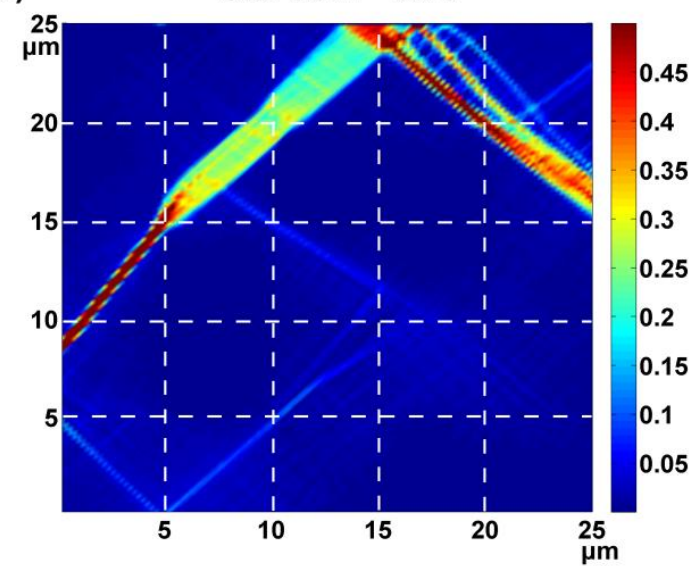

(d)

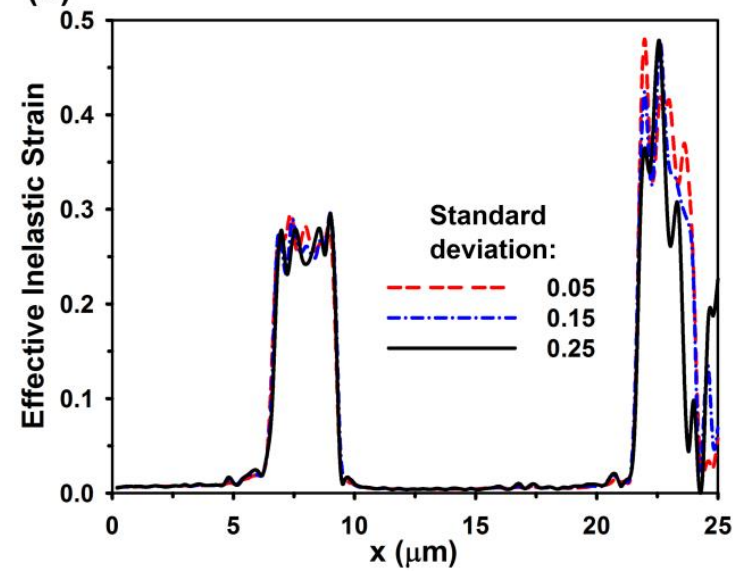

Figure 19. (a-c): Distribution of effective inelastic strain for different degrees of scatter of the spatially non-uniform initial interstitial loop density. (d): Inelastic strain profile along a horizontal section at $y=18 \mu \mathrm{m}$ in the material.

The overall localization patterns are qualitatively similar in all three microstructures, suggesting that the initial crystallography primarily governs the spatial distribution of localization. As the standard deviation increases from 0.05 to 0.25 in Figs. 19 (a)-(c), regions of 
micro-texture (in terms of inelastic strain) are observed. Slip localization, in the present case, takes place along multiple parallel paths due to trickling of mobile dislocations through the nonuniform defect field, thus giving rise to the observed micro-texture. Further, Fig. 19 (d) shows that the inelastic strain profile is jagged along the localized deformation regions. This suggests that the 'apparent' shear bands in Figs. 19 (a)-(c) are in fact composed of multiple localization pathways (or dislocation channels). The width of each of these channels is limited by the element size $(200 \mathrm{~nm})$ and varies from 200-400 nm (i.e., 1-2 elements). Due to their spatial proximity, interaction and coalescence of localization pathways may also contribute to the 'apparent' widening of dislocation channels discussed in Section 3.5.2.1, in addition to the cross-slip mechanism. As discussed in Zbib and Aifantis [142], if the spacing between two shear bands is less than a characteristic length scale, they may coalescence into one. More importantly, the inelastic strain pattern at $x \approx 22 \mu \mathrm{m}$ in Fig. 19 (d) clearly shows that as the degree of scatter increases, the localization profile becomes sharper, i.e., the inelastic deformation has a higher to tendency to localize within a single dislocation channel. In other words, scatter in the initial defect density helps regularize the dislocation channel width.

\subsubsection{Non-local model considerations}

We have reiterated throughout this Chapter that mesh dependence of the local constitutive framework stipulates that the dislocation channel width is inevitably governed by the minimum FE size. While a non-local constitutive framework seems to be the obvious approach to address this mesh dependence issue and model the inherent physical length scale, there are problems associated with the scale at which the dislocation channeling phenomena occurs.

As mentioned in Section 3.5.6, the dislocation channel width is governed by the mean

free path of mobile dislocations, $l_{d} \propto 1 / \sqrt{\rho_{M}}$, which trickle through the field of interstitial loops. This length scale may be accounted for by considering gradients of either the dislocation density or the inelastic strain. Aifantis and co-authors [142-147] have derived analytical conditions for the instability of a perturbed viscoplastic solid (localization is a type of material instability) by considering higher order gradients of either of these state variables in the evolution of flow stress. Based on linear and non-linear stability analyses of a simplified solid, they derived solutions for the size (width) and periodicity (spacing) of the localized regions. A similar gradient-based constitutive model may be used in our spatially resolved numerical finite element calculations to model the inherent length scale of localization. Though computationally intensive, this method is not entirely unfeasible.

However, as mentioned earlier, the length scale governing dislocation channel width is coincidental with the lower limit of continuum field approximation of continuously distributed dislocations (cf. [133]) in the present case. This stipulates that the minimum mesh size be of the 
order of 100-200 nm in our continuum field finite element calculations. Even if we were to use a gradient-based non-local model, it would be physically unreasonable to use a mesh resolution much further below $200 \mathrm{~nm}$. Hence, there is no significant advantage gained by using non-local continuum plasticity equations to model dislocation channels. In our simulations, the mesh size serves as the length scale governing dislocation channel width.

\subsection{Summary}

A continuum crystal plasticity framework has been used to model the localization behavior in irradiated bcc ferritic/martensitic steels, and results compared to available experimental data. Our simulations have been able to qualitatively capture majority of the microstructure evolution characteristics observed in experiments. The validity of the cross-slip model and its effect on the widening of dislocation channels in irradiated bcc materials has been examined. Effect of the defect annihilation model on lowering the flow stress and widening of dislocation channels has been studied. The cross-slip model and the flow softening model are essential to simulate the deformed microstructure of irradiated bcc metals and control the degree of localization in terms of the width of the dislocation channels.

It has been found that the maximum inelastic strain in channels is a linear function of the radiation dpa dose for the same initial crystallographic orientation distribution of grains. It has also been found that the localization behavior depends strongly on the crystallographic orientation distribution of grains. Further, the dislocation channel width is controlled by the mean free path of dislocations, $l_{d}$, and the channel spacing is controlled by the grain size. These parameters define the characteristic length scales in the localization phenomena in tensile deformation of irradiated metals.

Finally, it is imperative to note the differences between prior FE-based continuum modeling of flow localization in irradiated materials and the present work. Most of the prior FE modeling efforts [148-150] have explicitly modeled dislocation channels in the material, i.e., explicitly embedded 'softer' shear bands in a 'harder' matrix. In contrast, we started out with a bulk consisting of uniformly distributed irradiation-induced defects and let the microstructure evolve according to constitutive rules based on the physics of the material behavior under mechanical loading. Shear bands (dislocation channels) with higher accumulated inelastic stains (and lower defect densities) were found to form implicitly in the microstructure as a result of the "constitutive" material softening effects triggered by intergranular heterogeneity, combined with the flow softening arising from annihilation of irradiation-induced defects. More recently, Barton et al. [15] and Po and Ghoniem [151] have modeled flow localization in irradiated metals using continuum constitutive equations. As in our model, both these works have used phenomenological descriptions of the reduction in density of the irradiation-induced defects, thus allowing localization pathways to form. Interestingly, Po and Ghoniem [151] also found that 
shear localization is promoted by using a heterogeneously distributed initial density of irradiation-induced defects.

The present modeling approach serves the objective of parametric exploration of the underlying mechanisms governing localized deformation, and relating these to the deformation behavior expected for various mechanical and material loading histories (e.g., in-reactor tensile tests with continued generation of point defect clusters). Future work will focus on modeling these experiments. 


\section{CHAPTER 4}

\section{MODELING THE EFFECT OF FLOW LOCALIZATION ON FAILURE INITIATION AHEAD OF A SHARP NOTCH}

\subsection{Introduction}

Failure in irradiated alloys correlates to the flow localization along dislocation channels. Dislocation channels have been found to be more dense near the fracture surfaces, and the channel spacing decreases by more than an order of magnitude from that in the uniformly deformed region [75]. In terms of mechanical properties, the fracture toughness has been found to universally decrease with increasing radiation dose under quasi-static loading $[2,41,93,152]$. Fracture has been found, in many cases, to propagate along the 'faceted' interfaces of dislocation channels with the uniformly deformed bulk [109,153]. Further, intergranular cracks have been found to initiate at the intersections of dislocation channels and grain boundaries [154]. Inability of the grain boundaries (GBs) to accommodate highly localized strains from dislocation channels has been suggested as one of the dominant factors contributing to irradiation assisted intergranular stress corrosion cracking [155].

These experimental observations motivate the present study of flow localization and failure initiation ahead of a sharp notch in irradiated bcc materials by coupling crystal plasticity with nucleation and growth of damage. A void nucleation and growth-based continuum damage framework is developed in this Chapter and coupled to the existing defect density-based crystal plasticity framework to explore dislocation channel patterns, and void nucleation and growth in the regions of high stress triaxiality ahead of the notch. Effects of loading rate and temperature on failure initiation are studied. The framework is also used to predict the crack growth resistance and fracture toughness of irradiated specimens.

\subsection{Background}

\subsubsection{Flow localization and ductile failure in irradiated metals}

Failure in irradiated metals is triggered, in many cases, by plasticity mismatch at the interface of dislocation channels with the uniformly deformed bulk. The fracture surface of a ferritic/martensitic Eurofer-97 steel, irradiated at $300^{\circ} \mathrm{C}$, shows evidence of transgranular 'zigzag' fracture, and the dimples associated with ductile failure become more shallow with increasing radiation dose, suggesting pathways of localized shear, in particular [109]. The 'zigzag' fracture pattern suggests an arbitrary crack path along intertwined dislocation channels 
in the deformed material. It was proposed [109] that plasticity mismatch, at the interface of the 'hard' matrix and the 'soft' dislocation channel, promotes void nucleation, growth and coalescence, ultimately resulting in failure. It was also found [109] that the crack resistance of the quasi-statically loaded irradiated material degrades significantly as compared to the

unirradiated material. This was attributed to the occurrence of mixed mode (Mode I + II) fracture ahead of the crack tip. However, the crack resistance was found to increase under dynamic loading and was comparable to that of the virgin, unirradiated material.

Observation of 'shallow' ductile fracture was also reported for irradiated $9 \mathrm{Cr}-1 \mathrm{Mo}$ martensitic steel at both room temperature and at $250^{\circ} \mathrm{C}$, along with evidence of secondary cracks, which were not present in the unirradiated specimens [156]. Further, evidence of limitedductility 'channel' fracture with separation of fracture surfaces along localized-shear dislocation channel paths has been found in 304 stainless steel [153], 321 austenitic steel [152], and 308 and 316 stainless steel [157] at elevated temperatures and high radiation doses. Fracture surfaces show relatively flat facets along dislocation channels that intersect and are intertwined with other channels [153], akin to the 'zigzag' fracture [109] mentioned earlier, but without any dimples. This 'channel' fracture mechanism may be considered as a limiting case of transgranular ductile failure along dislocation channels in irradiated metals with little or no dimple formation.

Note that failure in irradiated materials does not always occur by ductile fracture. Although the quasi-static fracture toughness of metals has been universally found to degrade as a result of radiation, the failure mode is temperature dependent, with brittle cleavage fracture occurring at low test temperatures [3] and an increase in the apparent ductile-brittle transition temperature (DBTT) with increasing radiation $[6,158,159]$. In a recent study [160], it has been shown that the irradiation temperature has a dominant effect on the fracture toughness, as opposed to irradiation dose, in the regime of moderate to high radiation doses (3-148 dpa). Brittle fracture was observed when the ferritic/martensitic HT9 steel was irradiated at temperatures below $400^{\circ} \mathrm{C}$, while ductile fracture was observed when the irradiation temperature was higher [160].

\subsubsection{Models for void nucleation and growth}

Ductile failure occurs by nucleation, growth and coalescence of voids subsequent to significant plastic deformation in metals [161]. In unirradiated metals, nucleation of voids generally occurs at the interfaces of second phase particles and inclusions. In the absence of second phase particles, voids may nucleate by the condensation/clustering of vacancies in regions of high plastic deformation [162]. Under the influence of high plastic strain and hydrostatic stress (developed due to plastic instability during tensile loading, for example), these voids grow rapidly and coalesce, thus forming macroscopic flaws or cracks. 
The physics of void nucleation has been studied using the classical theory of heterogeneous nucleation at interfaces (GBs, inclusions) [163-168]. In these studies, it is implicitly assumed that the driving force for void nucleation is derived from the condensation of supersaturated vacancies (cf. [166,169]), while not explicitly tracking the vacancy concentration in the material. The energy barrier and critical radius for void nucleation was calculated given the applied normal stress and interfacial energy at GBs and second phase particles [163,164], and also considering local internal stresses due to the presence of voids [166]. A major conclusion from all these calculations was that high local stress concentration, more specifically high tensile normal stresses, are necessary for voids to nucleate. Based on experimental observations of voids in 304 stainless steel under creep loading [167], it was also concluded that many of the voids were formed in the material prior to mechanical loading, possibly during primary forming processes, thus bypassing the requirement for the presence of high stress concentration for voids to nucleate.

It should be noted that majority of the studies mentioned here concern intergranular fracture under quasi-static tensile or creep loading. In the context of the present problem, heterogeneous void nucleation is of interest because localization of deformation along dislocation channels in irradiated materials leads to the formation of interfacial regions with plasticity mismatch within the grains. In the regions of high stress concentration (e.g., ahead of a sharp notch or crack-tip), nucleation and growth of voids would presumably be promoted at such interfaces, ultimately leading to transgranular 'zigzag' fracture [109] or 'channel' fracture [153].

The problem of void growth has received much attention in the past, especially in the context of correlating void growth with macroscopic applied stress and plastic strain. The mechanics of void growth is generally studied using/extending models developed most notably some 30-40 year ago [170-175]. The influence of stress triaxiality and plastic strain on the growth rate of an isolated cylindrical void was first studied by McClintock [170]. Analytical solutions of the growth of an isolated spherical void in a perfectly plastic material by Rice and Tracey [171] showed an exponential dependence of the growth rate on the stress triaxiality ratio. Gurson [172] considered material softening as a result of void growth and developed a void porosity-dependent, and hydrostatic stress-dependent plastic potential function for a cell containing a void. This model was further extended to a periodic array of cells containing voids in a hardening material by Tvergaard $[173,174]$ and later modified by Tvergaard and Needleman [175] to account for void coalescence once a critical porosity is reached. More recently, models for the micromechanics of void growth and coalescence have also been developed and compared to experiments by Benzerga and co-authors [176-178]. A review of the current advances in continuum modeling of void phenomena (growth and coalescence) and associated implications for fracture is given by Besson [179]. 
However, these models are mostly phenomenological in nature and focus on strain-driven growth. Void growth by vacancy diffusion mechanisms has also been studied $[162,163,180$ 187]. It is generally agreed $[162,183,184,186,187]$ that vacancy surface diffusion and GB diffusion (where present) are the dominant mechanisms contributing to void growth in a supersaturated field of vacancies, as may arise in the case of irradiated metals. Among these studies, the work of Cuitiño and Ortiz [162] is of particular interest in that it employs a dislocation density based crystal plasticity framework to model vacancy supersaturation and void growth in fcc metals subject to plastic deformation.

In this Chapter, a continuum damage framework is developed to model heterogeneous nucleation and growth of voids due to condensation of vacancies formed by excessive inelastic deformation. The damage framework is coupled with the existing dislocation density crystal plasticity framework to study flow localization and failure initiation ahead of a sharp notch in irradiated bcc metals.

\subsection{Model description}

The constitutive model framework considers the following physical phenomena:

(a) inelastic deformation of bcc materials in the field of irradiation-induced defects (primarily interstitial loops) that evolve with inelastic strain,

(b) vacancy supersaturation in the regions of localized inelastic deformation along dislocation channels,

(c) void nucleation by condensation/clustering of supersaturated vacancies in the regions of stress concentration, further promoted along dislocation channel interfaces (via plasticity mismatch with the surrounding interior) and GBs,

(d) void growth via diffusional transport of vacancies from the bulk to the void surface under the influence of multi-axial stress.

These phenomena principally account for the decrease in ductility and eventual failure of irradiated metals. While the flow localization associated with channel formation is modeled using the existing crystal plasticity framework, constitutive equations for items (b)-(d) above are developed in this Chapter, and coupled to the crystal plasticity model in terms of the field of vacancies in the material. Note that the ferritic/martensitic steels modeled in this work have high swelling resistance; voids/vacancy loops are not generally observed post-irradiation, even with transmission electron microscopy [24]. Accordingly, it is assumed here that no voids are formed due to irradiation; rather they are assumed to form by vacancy condensation subsequent to inelastic deformation. 


\subsubsection{Crystal plasticity framework}

The coupled crystal plasticity-damage framework uses the void number density, $N_{V}$, and the corresponding mean void radius, $r_{V}$, as state variables, in addition to the ISVs defined earlier. Note that while the dislocation densities, $\rho_{M}^{\alpha}$ and $\rho_{I}^{\alpha}$, and the interstitial loop density, $N_{i}^{\alpha}$, and loop size, $d_{i}^{\alpha}$, are defined at the level of slip systems, $N_{V}, r_{V}$, and the interstitial, $c_{i}$, and vacancy concentrations, $c_{v}$, are defined at the mesoscale. Constitutive equations for the finite deformation crystal plasticity framework (item (a) above) were developed in Chapters 2 and 3 .

In this Section, we will briefly summarize the cross-slip and dislocation dipole annihilation models and the corresponding rate of vacancy generation due to inelastic deformation (item (b) above).

\subsubsection{Vacancy supersaturation due to inelastic deformation}

Cuitiño and Ortiz [162] developed a model for vacancy formation due to inelastic deformation in a dislocation density based crystal plasticity framework for fcc materials. They assumed that intersection jogs, formed during dislocation glide and cross-slip, leave behind a trail of vacancies during their subsequent motion. The formation of vacancies by edge dislocation dipole annihilation during cyclic deformation of fcc metals was also modeled by Repetto and Ortiz [188] to predict fatigue crack initiation. Based on similar concepts, we assume that vacancies are formed by cross-slip of dislocations, and by mutual annihilation of dipoles within a critical capture radius of the associated glide dislocations.

The cross-slip model [16] assumes that mobile screw dislocations from slip system $\alpha$ migrate to slip plane $\chi$, which has the lowest stress-biased activation energy barrier, $\left(\tau^{*}-\left|\tau^{\chi}\right|\right) V_{a}$, amongst all possible slip systems $\alpha \in \Theta$, such that all slip systems of subset $\Theta$ have the same Burgers vector direction. Here, $\tau^{*}$ is the critical activation stress for double-kink nucleation, $\tau^{\chi}$ is the resolved shear stress on slip system $\chi$ and $V_{a}$ is the activation volume for cross-slip. Accordingly, the rate of migration of mobile screw dislocations from slip system $\alpha$ is given as

$$
\dot{\rho}_{c s}^{\alpha}=-k_{c s} \exp \left(-\frac{\left(\tau^{*}-\left|\tau^{\chi}\right|\right) V_{a}}{k T}\right) \rho_{M}^{\alpha}\left|\dot{\gamma}^{\alpha}\right|
$$

where $k_{c s}$ is a material constant, $k$ is Boltzmann's constant, $T$ is absolute temperature, and $\dot{\gamma}^{\alpha}$ is the crystallographic shearing rate on slip system $\alpha$. The corresponding rate of accumulation of 
mobile screw dislocations on slip system $\chi$ with the lowest activation barrier for cross-slip is given as

$$
\dot{\rho}_{c s}^{\chi}=-\sum_{\alpha \in \Theta, \alpha \neq \chi}-\dot{\rho}_{c s}^{\alpha}=\sum_{\alpha \in \Theta, \alpha \neq \chi} k_{c s} \exp \left(-\frac{\left(\tau^{*}-\left|\tau^{\chi}\right|\right) V_{a}}{k T}\right) \rho_{M}^{\alpha}\left|\dot{\gamma}^{\alpha}\right|
$$

This migration rate, when multiplied by the Burgers vector, $b$, and the average line length of mobile dislocations, $l_{M}^{\alpha} \approx 1 / \sqrt{\rho_{M}^{\alpha}}$, gives the number of migration events per unit time. The corresponding rate of vacancy generation due to cross-slip is obtained as the product of the migration events per unit time with the atomic density $N_{0}=1 / \Omega$, i.e.,

$$
\left.\dot{c}_{v}^{\alpha}\right|_{c s}=N_{0} b l_{M}^{\alpha}\left|\dot{\rho}_{c s}^{\alpha}\right|=\frac{b l_{M}^{\alpha}}{\Omega} k_{c s} \exp \left(-\frac{\left(\tau^{*}-\left|\tau^{\chi}\right|\right) V_{a}}{k T}\right) \rho_{M}^{\alpha}\left|\dot{\gamma}^{\alpha}\right|
$$

where $\Omega$ is the atomic volume.

Mutual annihilation of mobile dislocation dipoles within a critical capture radius, $R_{c}$, of the gliding mobile dislocations is accounted for by the relation $[65,68,188]$

$$
\dot{\rho}_{\text {mutual }}^{\alpha}=-\frac{2 R_{c}}{b} \rho_{M}^{\alpha}\left|\dot{\gamma}^{\alpha}\right|
$$

where the factor of two accounts for the fact that two mobile dislocations with opposite Burgers vectors are annihilated via this mechanism. The total number of mutual annihilation events per unit time is obtained as half the product of the mutual dislocation annihilation rate with the Burgers vector and the capture radius, i.e., $b R_{c}\left|\dot{\rho}_{\text {mutual }}^{\alpha}\right| / 2[65,188]$. Following a similar procedure as in Eq. (4.3), the corresponding vacancy generation rate is given as

$$
\left.\dot{c}_{v}^{\alpha}\right|_{\text {mutual }}=N_{0} \frac{b R_{c}}{2}\left|\dot{\rho}_{\text {mutual }}^{\alpha}\right|=\frac{R_{c}^{2}}{\Omega} \rho_{M}^{\alpha}\left|\dot{\gamma}^{\alpha}\right|
$$

The factor of two here avoids double counting of the dislocation annihilation events. The total vacancy generation rate due inelastic deformation on all slip systems is thus given as

$$
\left.\dot{c}_{v}\right|_{i n}=\left.\sum_{\alpha, \alpha \neq \chi} \dot{c}_{v}^{\alpha}\right|_{c s}+\left.\sum_{\alpha} \dot{c}_{v}^{\alpha}\right|_{\text {mutual }}
$$

As a result, the total vacancy evolution rate (given in Eq. (2.10)) is modified to reflect the vacancy generation rate due to inelastic deformation, i.e.,

$$
\dot{c}_{v}=K_{0}-K_{i v} c_{i} c_{v}-\sum_{\alpha} K_{v s}^{\alpha}\left(\rho_{M}^{\alpha}+\rho_{I}^{\alpha}\right) c_{v}+\left.\dot{c}_{v}\right|_{i n}
$$

where $K_{0}, K_{i v}$ are the defect pair creation and recombination rates, respectively, and $K_{v s}^{\alpha}$ is the vacancy-dislocation reaction rate coefficient. Except for $\left.\dot{c}_{v}\right|_{i n}$, the other terms in Eq. (4.7) will be dominant mainly under concurrent irradiation. 


\subsubsection{Damage framework}

A continuum damage approach is used to model the evolution of an isotropic damage variable, $\phi$, in the material. Using the concept of effective stress, i.e., $\tilde{\sigma}=\sigma /(1-\phi)$, and the strain equivalence principle (cf. [189]), the elastic stiffness of the damaged material is given as

$$
\tilde{C}=(1-\phi) C
$$

where $\boldsymbol{C}$ is the elastic stiffness of the undamaged material and $\tilde{\sigma}$ is the effective stress in the damaged material. Here, $\phi$ is the volume fraction of voids. In what follows, the voids are assumed to be spherical in shape, and their volume number density, $N_{V}$, and mean radius, $r_{V}$, are used as ISVs in this damage framework.

\subsubsection{Void nucleation model}

Based on the classical theory of void nucleation due to vacancy condensation, the steady state nucleation rate of voids that are each composed of $j$ vacancies is given as $[6,169]$

$$
\dot{N}_{V}^{j}=Z \beta_{j} N_{V}^{p} \exp \left(-\frac{\Delta G_{j}}{k T}\right)
$$

where $Z$ is the dimensionless Zeldovich factor, $\beta_{j}$, is the rate of impingement of vacancies on a void nucleus having $j$ vacancies, $N_{V}^{p}$ is the number of potential nucleation sites, and $\Delta G_{j}$ is the Gibbs free energy change associated with the formation of a void nucleus having $j$ vacancies. A relation between the radius, $r_{V}$, of the void and the number of vacancies, $j$, in the void can be obtained by equating their respective volumes, i.e.,

$$
\frac{4 \pi}{3} r_{V}^{3}=j \Omega \Leftrightarrow r_{V}=\left(\frac{3 j \Omega}{4 \pi}\right)^{1 / 3}
$$

where $\Omega$ is the volume of a single vacancy. An implicit assumption, of course, is that the voids are composed only of vacancies; there are no gas atoms present within the voids. Accordingly, the free energy change associated with the formation of a spherical void composed of $j$ vacancies under the influence of local tensile hydrostatic stress, $\sigma_{H}$, is given as

$$
\Delta G_{j}=-j \sigma_{H} \Omega+(4 \pi)^{1 / 3}(3 j \Omega)^{2 / 3} \Delta \Upsilon=-\left(\frac{4 \pi}{3} r_{V}^{3}\right) \sigma_{H}+\left(4 \pi r_{V}^{2}\right) \Delta \Upsilon
$$

where $\Delta \Upsilon$ is the associated interfacial energy change, and is given as the difference between the surface energy of new void surfaces created and the surface energy of interfaces annihilated as a result of void formation. The present framework considers both heterogeneous void nucleation at the dislocation channel-induced plasticity mismatch interfaces and homogeneous void nucleation in the homogeneously deformed grain interiors; $\Delta \Upsilon$ would vary accordingly depending on 
whether the nucleation site is at a dislocation channel interface or a GB interface $\left(\Delta \Upsilon=\Delta \Upsilon_{\text {het }}\right)$, or in the homogeneous interior $\left(\Delta \Upsilon=\Delta \Upsilon_{h o m}\right)$. The critical number of vacancies, $j^{*}$, required to form a void nucleus of radius, $r_{V}^{*}$, is obtained from the stationary point of $\Delta G_{j}$, i.e.,

$$
\frac{\partial \Delta G_{j}}{\partial j}=0 \Rightarrow j^{*}=\frac{32 \pi}{3 \Omega}\left(\frac{\Delta \Upsilon}{\sigma_{H}}\right)^{3} \Leftrightarrow r_{V}^{*}=\frac{2 \Delta \Upsilon}{\sigma_{H}}
$$

and the associated critical free energy change is

$$
\Delta G^{*}=\frac{16 \pi}{3} \frac{\Delta \Upsilon^{3}}{\sigma_{H}^{2}}
$$

The non-equilibrium Zeldovich correction factor, $Z$, accounts for fluctuations between the subcritical and supercritical nuclei [165], and is given as [6,169]

$$
Z=\left(-\frac{1}{2 \pi k T}\left[\frac{\partial^{2} \Delta G_{j}}{\partial j^{2}}\right]_{j^{*}}\right)^{1 / 2}
$$

The impingement rate of vacancies on the void nucleus, $\beta_{j}$, depends on the probability of finding vacancies within an atomic layer, $\Omega^{1 / 3}$, of the void surface, i.e., $\left(4 \pi r_{V}^{2}\right) \Omega^{1 / 3} / \Omega$, and the jump frequency of vacancies, given by $D_{v} / \Omega^{2 / 3}[165]$. Here, $D_{v}$ is the vacancy diffusivity. Accordingly, the impingement rate is given as

$$
\beta_{j}=\frac{4 \pi r_{V}^{2}}{\Omega^{2 / 3}} \frac{D_{v}}{\Omega^{2 / 3}}
$$

From Raj and Ashby [163], the number of potential nucleation sites per unit volume, $N_{V}^{p}$ , is related to the available volume for void nucleation. Hence, it depends on the damage parameter, $\phi$, and the current volume, $j \Omega$, of the void nucleus, i.e.,

$$
N_{V}^{p}=\frac{1-\phi}{j \Omega}
$$

As a result of nucleation of voids due to vacancy condensation, the vacancy concentration in the material is depleted. Based on the steady state void nucleation rate given in Eq. (4.9) and the critical number of vacancies required to nucleate the voids given in Eq. (4.12), the corresponding mean field vacancy absorption rate is given by

$$
\left.\dot{c}_{v}\right|_{V} ^{n}=j^{*} \dot{N}_{V}^{j^{*}}
$$

This framework tracks the number density and radii of voids at each material point. However, as a result of simultaneous nucleation and growth events, there would exist a distribution of void sizes at any instant of time. Keeping track of such a void size distribution for each material point in a crystal plasticity framework is computationally expensive, given that the 
slip system-ISVs must be tracked as well. Hence, we track only the average void radius at each material point, which is calculated by conserving the volume of the pre-existing and newly nucleated voids, i.e.,

$$
\left\langle r_{V}\right\rangle_{t}=\frac{\left(\left.\left(\left.\dot{N}_{V}\right|_{t} \Delta t\right) r_{V}\right|_{t} ^{3}+\left.N_{V}\right|_{t-1}\left\langle r_{V}\right\rangle_{t-1}^{3}\right)^{1 / 3}}{\left.\dot{N}_{V}\right|_{t} \Delta t+\left.N_{V}\right|_{t-1}}
$$

where subscripts $t$ and $t-1$ in the above equation refer to values of the variable at the respective time steps, $\Delta t$ is the time interval between these time steps, and values within \langle\rangle represent mean values of the respective variables. For brevity, the average void radius at the current time step, $\left\langle r_{V}\right\rangle_{t}$, will be simply referred to as $r_{V}$ in the following Sections, unless mentioned otherwise.

Note that effect of the presence of interstitials on void nucleation and growth is neglected in the present work. This is reasonable given the fact that interstitials, though present due to prior irradiation, may be neglected in comparison to the significantly larger number of vacancies generated during inelastic deformation.

\subsubsection{Void growth model}

As mentioned earlier, surface diffusion is one of the dominant mechanisms contributing to void growth (in the absence of GB diffusion). Void growth may also be reaction rate controlled (cf. [6]). Reaction rate controlled processes are those in which no macroscopic concentration gradients of the reactants exist; rather, these processes are governed by the 'sink strengths' of the respective reactants. Initially when $r_{V} \leq 1 \mathrm{~nm}$, void growth may be reaction rate limited [6]. As the voids increase in size, the growth rate is controlled by the diffusive flux of vacancies from the surrounding material. As outlined in Was [6], a mixed void growth rate equation may be used to reflect contributions from each of these mechanisms. However, for simplicity, we focus on modeling just diffusion controlled void growth. This is reasonable given the fact that initial reaction rate controlled void growth in the field of supersaturated vacancies will be swift, and diffusion controlled growth is the rate limiting step (a similar assumption has also been made in $[162,187,190])$. Note that void growth is further accelerated in the regions of heavy inelastic deformation due to vacancy pipe diffusion along dislocations [162,188,191].

From Was [6], assuming steady state diffusion in an infinite field of vacancies, the absorption rate of vacancies for void growth is calculated using a standard rate theory approach, i.e.,

$$
\left.\dot{c}_{v}\right|_{V} ^{g}=4 \pi r_{V} D_{v} N_{V}\left(c_{v}-c_{v}^{V}\right) ; c_{v}>c_{v}^{V}
$$


where $c_{v}^{V}$ is the vacancy concentration at the void surface. This expression is obtained by solving the steady state diffusion of vacancies to a spherical void lying within a larger spherical shell, which is an infinite vacancy source. Applying conservation to the vacancy change rate within voids, the equivalent expression for uniform growth rate of voids is given by [6]

$$
N_{V} \dot{V}_{V}=N_{V}\left(4 \pi r_{V}^{2} \dot{r}_{V}\right)=\left.\dot{c}_{v}\right|_{V} ^{g} \Omega \Rightarrow \dot{r}_{V}=\frac{\Omega}{r_{V}} D_{v}\left(c_{v}-c_{v}^{V}\right) ; c_{v}>c_{v}^{V}
$$

The vacancy concentration at the void surface, $c_{v}^{V}$, is a function of the force balance between the external tensile hydrostatic stress, $\sigma_{H}$ (promoting growth), and the inward pressure due to surface tension, $2 \gamma_{V} / r_{V}$ (resisting growth). Following a similar procedure as in Was [6], $c_{v}^{V}$ as a function of the net resultant force due to surface tension and hydrostatic stress, is given by

$$
c_{v}^{V}=c_{v}^{0} \exp \left(\frac{\Omega}{k T}\left(\frac{2 \gamma_{V}}{r_{V}}-\sigma_{H}\right)\right)=\frac{1}{\Omega} \exp \left(-\frac{E_{v}^{f}-T S_{v}^{f}}{k T}\right) \exp \left(\frac{\Omega}{k T}\left(\frac{2 \gamma_{V}}{r_{V}}-\sigma_{H}\right)\right)
$$

where $c_{v}^{0}$ is the equilibrium vacancy concentration, $E_{v}^{f}$ is the vacancy formation energy, $S_{v}^{f}$ is the vacancy formation entropy, and $\gamma_{V}$ is the interfacial energy associated with creation of a new void surface. Note that this expression also gives the dependence of the void growth rate, $\dot{r}_{V}$, on $\sigma_{H}$. A higher value of $\sigma_{H}$ would lower $c_{v}^{V}$, thus increasing $\dot{r}_{V}$ (from Eq. (4.19)), and viceversa.

As mentioned earlier, vacancy diffusion is accelerated along dislocations (pipe diffusion) in the regions of excessive inelastic deformation. Based on the pipe diffusion model, the vacancy diffusion coefficient in the grain interior is a function of the dislocation density in the material, and is given as [191]

$$
D_{v}=D_{v}^{l}+b^{2}\left(\sum_{\alpha} \rho^{\alpha}\right) D_{v}^{\rho}
$$

where $D_{v}^{l}$ is the vacancy lattice diffusion coefficient, and $D_{v}^{\rho}$ is the vacancy pipe diffusion coefficient.

Note that the present framework models only the initial stages of failure due to diffusiondriven void growth. It is expected that strain-driven void growth and coalescence will dominate in the later stages. Strain-driven void growth may be modeled using the Rice and Tracey model [171], for example. Further, our model assumes that the voids are spherical in shape. Ellipsoidal void growth is generally expected at high stress triaxialities (cf. [179]), and has been studied in Gologanu et al. [192], for example. Future work may address these issues. 
Before moving further, it should be noted that the presence of voids is expected to cause additional resistance to the glide of dislocations. Accordingly, this is accounted by modifying the dispersed barrier hardening model for athermal slip resistance of dislocations (Eq. (2.5)) to the following form:

$$
s_{a}^{\alpha}=G b \sqrt{\sum_{\zeta=1}^{N_{s}} q_{\rho} A^{\alpha \zeta} \rho^{\zeta}+q_{i} N_{i}^{\alpha} d_{i}^{\alpha}+q_{V} N_{V} r_{V}}
$$

where $q_{V}$ is the void barrier strength. Note that while dislocations and interstitial loops are expected to resist dislocation glide at the level of slip systems, voids are not associated with a particular slip system and resist glide on all slip systems.

\subsubsection{Modeling simultaneous void nucleation and growth}

Void nucleation and growth are competitive processes. Initially, when there is little or no damage in the material, void nucleation would obviously occur preferentially over void growth. However, with increasing damage, void growth takes preference over nucleation as potential nucleation sites decay in number (modeled using Eq. (4.16)). A simple strategy is implemented here to model void nucleation and growth, decomposing it into a two-step process. It is assumed that following inelastic deformation, void nucleation occurs if possible. Criteria for critical void nucleation are outlined in the algorithm given in Fig. 20. Subsequent to nucleation, void growth is allowed to occur according to Eq. (4.20). Though this is a deterministic treatment of the problem of simultaneous void nucleation and growth, the nucleation rate is slowed down at higher damage values due to the lack of potential nucleation sites according to Eq. (4.16). Figure 20 presents the detailed algorithm for modeling void nucleation and growth and coupling it to the crystal plasticity framework. 


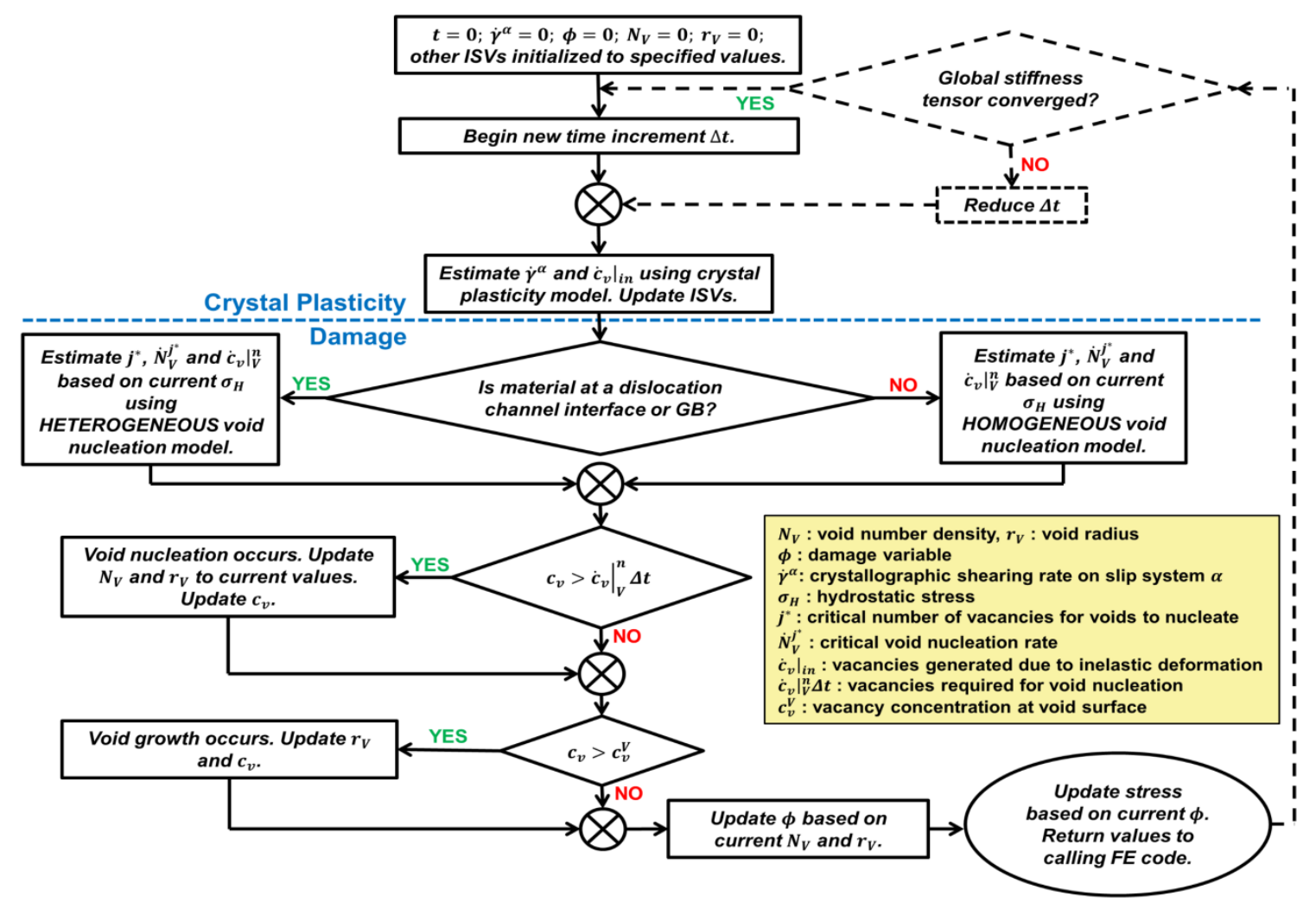

Figure 20. Algorithm for integration of void nucleation and growth-based damage model. Dashed black lines indicate operations performed by calling FE code.

\subsection{Model implementation}

\subsubsection{Loading and boundary conditions}

The 3D constitutive model is implemented in the FE program ABAQUS [86] as a User MATerial (UMAT) subroutine. Generalized plane strain elements are used along with fully 3D crystallography and the described constitutive model. The elements employ linear interpolation and reduced integration.

A single deep edge notch with semi-circular tip is created at mid-height on the side of a 800-800 $\mu \mathrm{m}$ simulation cell. A schematic of the loading and boundary conditions is shown in Fig. 21 (a). Regions in the immediate vicinity of the notch are modeled using the (incremental) coupled crystal plasticity-damage material behavior described in Section 4.3. The microstructure (courtesy Dr. J. Padbidri, Georgia Tech) is generated using an approximate Dirichlet (weighted 
Voronoi) tessellation achieved by packing of (randomly oriented) polygonal grains with Gaussian size distribution ( $25 \mu \mathrm{m}$ mean size), through random sequential addition. Deformation plasticity model with Ramberg-Osgood hardening behavior is assigned to the outer region (shaded in green) in Fig. 21 (a). Based on the dimensions of the respective regions indicated in Fig. 21 (a), a representative simulation cell has $\sim 45$ grains in the crystal plasticity domain enveloping the notch root. Different notch depths and radii are used in the simulations; these values are specified where needed.

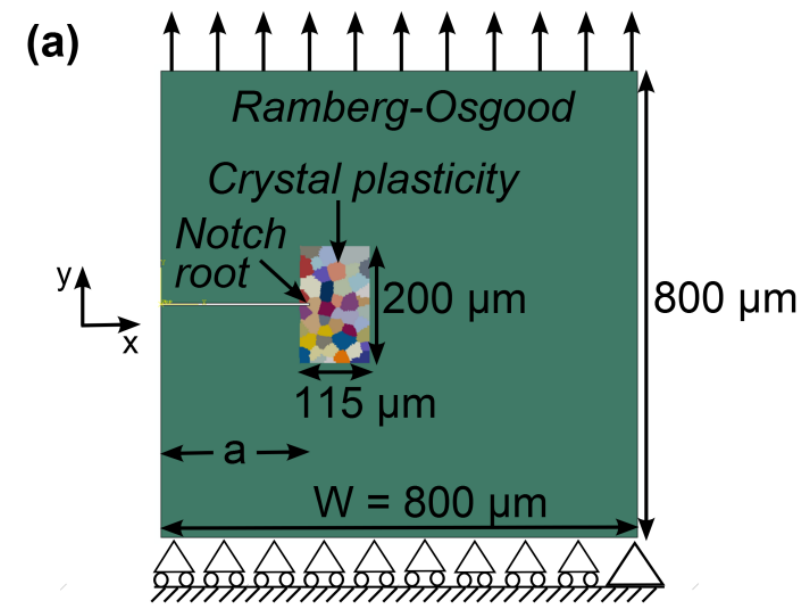

(b)

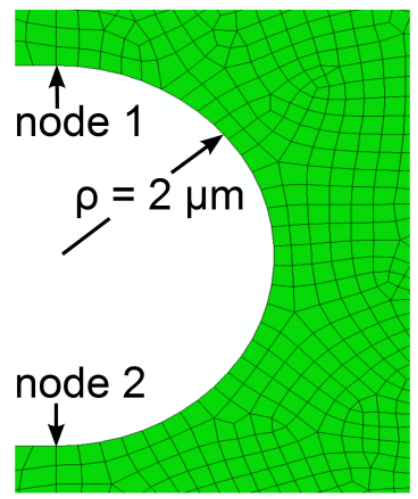

Figure 21. (a): Schematic of loading and boundary conditions. (b): Representative mesh ahead of a notch root of radius, $\rho=2 \mu \mathrm{m}$, having a minimum mesh size of $200 \mathrm{~nm}$.

The bottom edge of the 2D simulation cell is constrained along the loading direction, and the far right node of the bottom edge is constrained in both $\mathrm{x}$ and $\mathrm{y}$ degrees of freedom (to prevent rigid body rotation). Displacement-controlled tensile loading is applied on the top edge in the $y$ direction (remote applied straining), and the side edges of the simulation cell are traction-free. The simulation cell is loaded at quasi-static strain rate $\left(10^{-4} \mathrm{~s}^{-1}\right)$, unless mentioned otherwise.

The simulation cell is freely meshed in ABAQUS using quadrilateral and triangular elements. A mesh size of $200 \mathrm{~nm}$ is used in the crystal plasticity zone in regions in the immediate vicinity and up to $5 \mu \mathrm{m}$ away from the notch, with radially increasing mesh size up to $4 \mu \mathrm{m}$ in the crystal plasticity zone, and up to $15 \mu \mathrm{m}$ in the Ramberg-Osgood zone. A typical simulation cell has $\approx 15,000$ elements in the crystal plasticity zone, $\approx 5,000$ elements in the RambergOsgood zone. A representative mesh ahead of a deep notch with radius, $\rho=2 \mu \mathrm{m}$, is shown in Fig. 21 (b). This sharp notch effectively represents a crack.

Following Refs. [193,194], the crack tip displacement (CTD) computed as the net displacement between nodes 1 and 2 labeled in Fig. 21 (b), is used to characterize the driving 
force for fracture. The $C T D$ relates to the $J$-integral for stationary crack growth (cf. [161,195]), and is often used to quantify fracture. Also note that $C T D=\sqrt{C T O D^{2}+C T S D^{2}}$. Thus, $C T D$ is able to characterize failure under mixed mode (Mode I and Mode II) loading. Here, CTOD is the crack tip opening displacement, and $C T S D$ is the crack tip shear displacement.

\subsubsection{Algorithm for identifying dislocation channel interface and GB elements}

An essential step in modeling void nucleation and growth in the present framework is identifying whether a material point is in the homogeneously deformed bulk, dislocation channel interface, or at a GB. This is implemented in our FE simulations in the following fashion:

(a) A list of nearest neighbor elements is stored that lie within a cutoff radius, $1.5 d$ ( $d$ is the characteristic length of the material point, defined as the square root of the area of the respective element), of the centroid of the element in question.

(b) At each time step, the difference in effective inelastic strain, $\varepsilon_{\text {eff }}^{i}$, between the neighboring elements is computed. If the difference is greater than 0.05 , and $\varepsilon_{\text {eff }}^{i}$ is lower in the present FE than its neighbor, then the material point is at a dislocation channel interface.

(c) If the neighboring element belongs to a different grain, the material point is at a GB.

(d) If none of the above conditions are satisfied, the material point lies within the uniformly deformed bulk.

\subsubsection{Non-local vacancy concentration at interface elements}

This simple framework identifies interface elements along dislocation channels and GBs in the material. Heterogeneous void nucleation and growth processes are expected to be more prolific at these interface elements. Physically, the vacancy population at material points contiguous to the 'interface' should also contribute to the nucleation and growth of voids. This is implemented in our framework by taking a non-local estimate of the vacancy concentration at the interface elements. Using the nearest neighbor list computed in Section 4.4.2, the non-local vacancy concentration, $\left.c_{v}\right|_{n l} ^{i}$, at the interface element is calculated as the element area-weighted sum of vacancy concentration over all nearest neighbors, including the vacancy concentration at the interface element. If void nucleation and/or growth occurs at the interface element, vacancy concentration in the neighboring elements is depleted proportional to their contribution to $\left.c_{v}\right|_{n l} ^{i}$.

\subsubsection{Damage estimation}

The crystal plasticity and damage framework outlined here employs 3D crystallography and 3D constitutive equations. However, as explained in Section 4.4.1, the polycrystalline 
simulations are performed using generalized plane strain FEs, effectively treating polyhedral grains as columnar (polygonal cross-section in the plane). We employ a commonly used microstructure scaling relation, which relates the mean areal density, $\left\langle x^{A}\right\rangle$, of convex-shaped particles to the volume density, $x^{v}$, according to the relation

$$
\left\langle x^{A}\right\rangle=\langle D\rangle x^{V}
$$

where $\langle D\rangle$ is the mean caliper diameter of the particle, to find the areal density $\left(N_{V}^{A}\right)$ of voids across the material cross-section. Further, using Cavalieri's principle, the volume fraction of voids, $V_{V}$, is equal to the average area fraction of voids, $A_{A}$ (cf. [196]). Thus, the damage variable, $\phi$, measuring the volume fraction of voids, is given as

$$
\phi=V_{V}=A_{A}=\frac{N_{V}^{A} \pi r_{V}^{2}}{A_{0}}=\frac{\left(2 r_{V} N_{V}\right) \pi r_{V}^{2}}{A_{0}}
$$

where $A_{0}$ is the cross-sectional area of the initially undeformed material.

\subsubsection{Model parameters}

The framework is used to simulate the deformation behavior of Mod 9Cr-1Mo steel loaded in quasi-static tension. This steel has good radiation resistance and high temperature properties. The microstructure of $\mathrm{Mod} 9 \mathrm{Cr}-1 \mathrm{Mo}$ steel is mainly comprised of ferrite and martensite phases. It is assumed that the crystal structure of the subsequently tempered martensite phase is body-centered cubic (cf. [77]). Accordingly, the material is assigned a uniform bcc crystal structure with 24 slip systems (12 \{110\}-type and 12 \{112\}-type). To save computational costs, deformation along the $24\{123\}$ bcc slip systems is neglected for the example applications presented in this Chapter.

The crystal plasticity model was calibrated earlier [16,91] to match the experimental engineering stress-strain data of Mod 9Cr-1Mo steel [2] for different radiation and temperature histories. In addition to the parameter estimation described in Chapter 2, the void barrier strength, $q_{V}$, is assumed to have a value of unity. This follows the experimental observation that voids are the strongest obstacles in the path of gliding dislocations [41].

The damage model adds only four new parameters to the framework: $E_{v}^{f}, S_{v}^{f}, D_{v}^{\rho}$, and $\Delta \Upsilon$. The vacancy formation energy, $E_{v}^{f}$, and entropy, $S_{v}^{f}$, are taken from [82]. Studies have shown $[197,198]$ that the pipe diffusion coefficient, $D_{v}^{\rho}$, is $\sim 3-4$ orders of magnitude higher than the lattice diffusion coefficient in bcc-Fe at elevated temperatures, and even higher at low and intermediate temperatures. Accordingly, we assume that $D_{v}^{\rho}=10^{4} D_{v}^{l}$. The interfacial energy 
change associated with homogeneous void nucleation is $\Delta \mathrm{r}_{\text {hom }}=\gamma_{V}=1.95 \mathrm{Jm}^{-2}$ [66], where $\gamma_{V}$ is the surface energy of the material. The exact value of $\Delta \Upsilon_{h e t}$ is not entirely known. From [66], the grain boundary interfacial energy of $\mathrm{Fe}, \gamma_{G B} \approx 0.78 \mathrm{Jm}^{-2}$. Assuming that the energy of a dislocation channel interface is the same, the interfacial energy change associated with heterogeneous nucleation of a spherical void at an interface (in Eq. (4.12)) is ideally given by $\Delta \Upsilon_{h e t}=\gamma_{V}-\gamma_{G B} / 4$. This expression accounts for the fact that a void of surface area, $4 \pi r_{V}^{2}$, with interfacial energy, $\gamma_{V}$, is created by destroying a dislocation channel interface (or GB) of area, $\pi r_{V}^{2}$, with interfacial energy, $\gamma_{G B}\left(4 \pi r_{V}^{2}\right.$ is already factored out in the corresponding term in Eq. (4.12)). Substituting values of the respective parameters into the above expression, $\Delta \Upsilon_{h e t} \approx 1.75 \mathrm{Jm}^{-2}$. This value of $\Delta \Upsilon_{\text {het }}$ is too high to allow spherical void nucleation even at peak stress for stress triaxialities on the order of 3-3.5. Note that an assumption of spherical voids is used here just to simplify the constitutive equations; the voids are seldom spherical in nature. This is because a spherical shape has the highest contact angle $\left(90^{\circ}\right)$ at the void-GB interface (cf. [163]), and is the least energetically favorable. Contact angle-based shape factors of voids of various shapes and their effect on void nucleation and growth at different temperatures has been studied in detail by Raj and Ashby [163]. Based on their analysis of void nucleation at grain interfaces, plausible void-interface contact angles lie in the range $0^{\circ}-37^{\circ}$ for $\mathrm{Cu}$, meaning that the nucleated voids are essentially prolate spheroids with a high degree of eccentricity. The surface and interfacial energies of $\mathrm{Fe}$ are in the same range as $\mathrm{Cu}$, allowing us to extrapolate these results to the present case. Using the shape factors given in [163] and the contact angles mentioned above, $\Delta \Upsilon_{h e t} \leq 0.33 \mathrm{Jm}^{-2}$ for Fe. Note that the surface and interfacial energy values reported above are generally measured at $T>1000 \mathrm{~K}$. These values increase with decrease in temperature; at intermediate temperatures of $400-600 \mathrm{~K}$, the calculated value of $\Delta \Upsilon_{h e t}$ would be even higher. Accordingly, we have used a value of $\Delta \Upsilon_{\text {het }}$ in the range $0.32-0.38 \mathrm{Jm}^{-2}$ in our simulations. Effects of this parameter on damage initiation and the computed CTD are studied in Section 4.5.3.

As mentioned in Section 4.4.1, Ramberg-Osgood hardening behavior is assumed for the material far away from the notch in the context of a deformation plasticity model. Separate sets of Ramberg-Osgood model parameters, for different loading (temperature, strain rate and radiation dose) histories, were generated by fitting to the stress-strain response from the respective crystal plasticity simulations of smooth specimens with 125 , randomly oriented cubic grains of $30 \mu \mathrm{m}$ size, deformed uniaxially up to $1.5 \%$ engineering strain. 


\subsection{Results and discussion}

Post-irradiation tension test simulations are performed on the notched specimens at different test temperatures and loading rates. The following sections will first consider inelastic deformation and vacancy generation in the vicinity of the notch, and then describe the damage behavior.

\subsubsection{Channel formation and vacancy supersaturation}

Figure 22 shows the distribution of inelastic strain and the corresponding vacancy concentration ahead of a circular notch of radius, $\rho=2 \mu \mathrm{m}$, with notch depth, $a=250 \mu \mathrm{m}$, in the material irradiated to $2.9 \mathrm{dpa}$ and then strained normal to the notch to $0.7 \%$ remote applied engineering strain at $550 \mathrm{~K} . \Delta \Upsilon_{h e t}=0.38 \mathrm{Jm}^{-2}$ in these simulations. Figure 22 (a) shows that the deformation is primarily localized in a single grain ahead of the notch. As mentioned earlier, localization of deformation occurs via reduction in density of the irradiation-induced defects along these channels, thus reducing the flow stress. This has been modeled phenomenologically in Eq. (3.2) as a function of the interacting defect density and mobile dislocation density (cf. Chapter 3 for detailed description of the localization mechanism). Multiple, intertwined channels are formed in the regions of stress concentration in the immediate vicinity of the notch. Reorientation and bifurcation of channels at GBs is also observed. The spacing between channels here is much lower than computed previously for the case of localization in smooth specimens in Chapter 3. This is in agreement with experimental observation of higher density of dislocation channels near the fractured surface as compared to uniformly deformed regions [75]. We also found earlier in Chapter 3 that the dislocation channel spatial patterns are strongly dependent on the crystallography of the material. The width of modeled dislocation channels is limited by the minimum mesh size at $200 \mathrm{~nm}$, and is larger than the experimentally observed channel width of $50-200 \mathrm{~nm}[26,75,106,107]$. Further, the localized inelastic strain in channels $(\sim 0.6-1.4)$ is lower than that seen in experiments $(\sim 1.0-5.0)[96,134]$. However, we have verified that the simulated net localization of shear strain in the stress-strain response of polycrystalline, smooth specimen ensembles is comparable to experiments, and the product of the channel width with the localized strain within channels is more or less invariant (cf. [91]). A finer mesh may be used to achieve the experimentally observed channel widths and localized strains, albeit at the cost of significantly increased computational times. For the present qualitative study of dislocation channel patterns and failure initiation ahead of the notch root, a mesh resolution of $200 \mathrm{~nm}$ is deemed sufficient. 
(a)

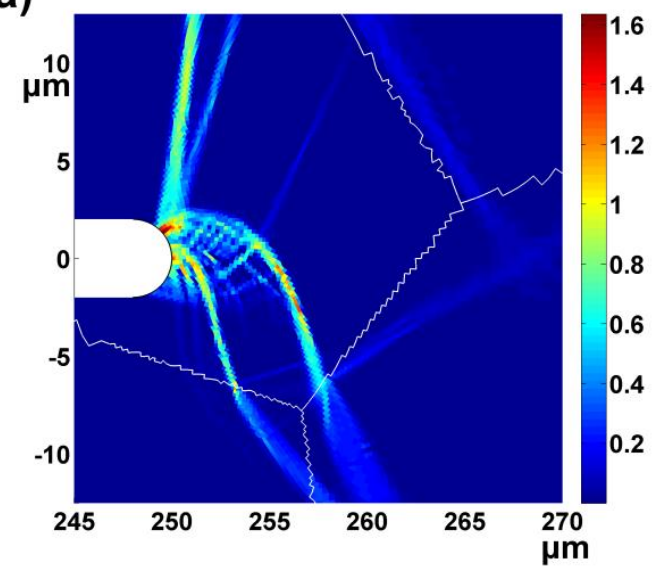

(b)

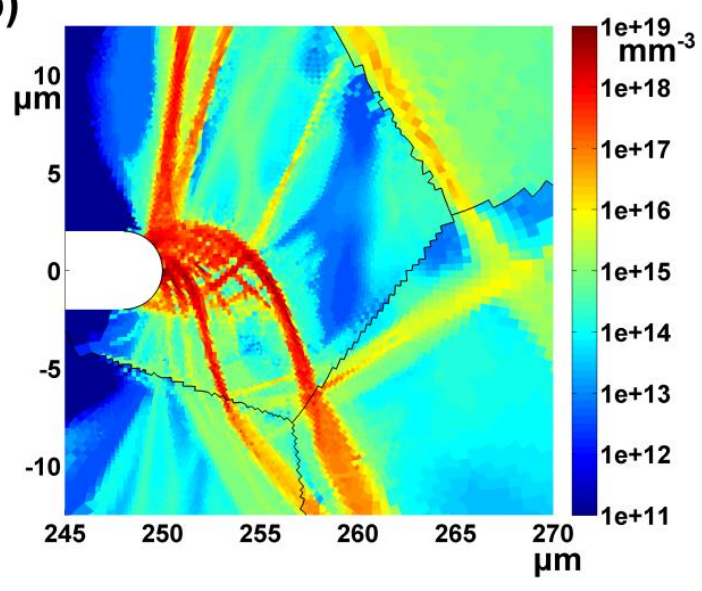

Figure 22. Distribution of (a): effective inelastic strain, and (b): vacancy concentration ahead of the notch after $0.7 \%$ applied strain. Grain boundaries are rendered. The material was irradiated to 2.9 dpa prior to tensile loading at $550 \mathrm{~K}$.

Figure 22 (b) shows that the vacancy concentration increases by 3-4 orders of magnitude in the regions of localized deformation, thus facilitating void nucleation and growth. The vacancy generation due to inelastic deformation has contributions from both dislocation cross-slip and annihilation of dislocation dipoles. We have verified that the vacancy contribution from these respective mechanisms is of the same order of magnitude using the model parameters specified in Table 1. As will be shown later, this vacancy concentration is in general sufficiently high to promote void nucleation and growth even at GBs ahead of the notch root, where localized deformation is not that prevalent.

\subsubsection{Damage evolution}

Figure 23 shows the distribution of voids (Fig. 23 (a): number density, 23 (b): radius) corresponding to the inelastic strain distribution shown in Fig. 22 (a). Void nucleation and growth is observed at multiple regions along the dislocation channel interfaces. In some cases, voids nucleate on both sides of a dislocation channel, for example in the regions A and B marked in Fig. 23 (a). The nature of void activity within the grain is consistent with the experimentally observed, faceted 'zigzag' fracture [109] along localized shear pathways. Strain-driven void growth and coalescence (not modeled here) would ultimately lead to transgranular fracture along these failure routes. Further, void activity is also prevalent at the intersection of dislocation channels with GBs ahead of the notch root. As mentioned earlier, incompatibility of highly localized strains along dislocation channels at their intersection with GBs is known to contribute to intergranular stress corrosion cracking in irradiated materials [155]. Note that the interfacial 
energy change associated with heterogeneous void nucleation was assigned to be the same for nucleation at both dislocation channel interfaces and GBs, i.e., $\Delta \Upsilon_{h e t}=0.38 \mathrm{Jm}^{-2}$.

(a)

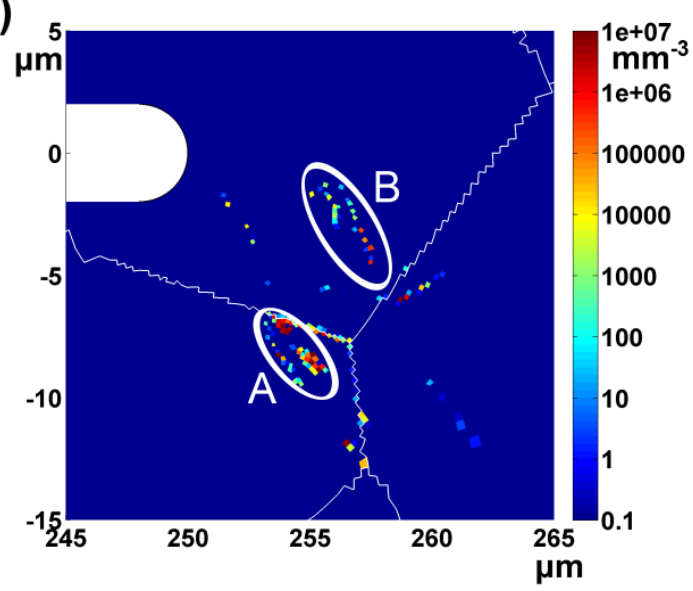

(b)

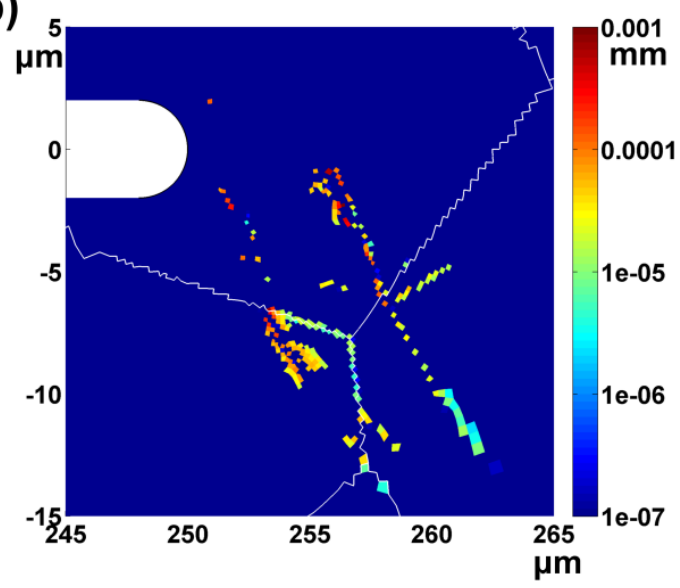

Figure 23. Distribution of (a): void number density, and (b): void radius corresponding to the effective inelastic strain in Fig. 22(a). See text for discussion of regions $A$ and $B$.

Figure 23 (a) shows that the density of voids goes up to $10^{7} \mathrm{~mm}^{-3}$, and the void radius up to $1 \mu \mathrm{m}$ in Fig. 23 (b). Correlating the void radius distribution to the vacancy concentration in Fig. 22 (b), it is found that the void radius is higher in regions of higher vacancy concentration. The void radius is in general higher along dislocation channel interfaces, compared to GBs. This is because the vacancy concentration is higher along the dislocation channel interfaces than GBs.

The evolution of damage (volume fraction of voids) for the same microstructure is shown in Fig. 24. Figure 24 (a) shows the evolution of total damage with applied strain. This is calculated as the total area-weighted damage over all elements in a semi-circular region of $25 \mu \mathrm{m}$ radius ahead of the notch root, i.e.,

$$
(\phi)_{t}=\frac{\sum_{i} \phi_{i} A_{i}}{\sum_{i} A_{i}}
$$

where $A_{i}$ is the area of the $i^{\text {th }}$ FE. The corresponding damage distribution at $0.45 \%, 0.55 \%$, and $0.70 \%$ strain is shown in Figs. 24 (b), 24 (c), and 24 (d), respectively.

As seen in Fig. 24 (a), damage initiates first at $\approx 0.32 \%$ applied remote strain in the present case. The evolution of damage is however not uniform; change in damage rate with applied strain is observed. This is associated with damage propagation along new region(s), and can be correlated to the corresponding damage distribution plots. Initially failure propagates mainly along the region $\mathrm{C}$ marked in Fig. 24 (b). At $0.55 \%$ strain, damage is also found to propagate along the new regions $\mathrm{D}$ and $\mathrm{E}$ marked in Fig. 24 (c). With further applied 
deformation, failure is again initiated along region F marked in Fig. 24 (d). Decrease in the damage rate, for example in the range 0.4-0.5\% applied strain in Fig. 24 (a), is due to relative saturation of damage in region $\mathrm{C}$, and consequently its inability to accommodate the applied deformation, promoting failure elsewhere.

Note that a maximum damage of 0.65 is allowed in each element. Excessive element distortion beyond this damage level leads to convergence issues in our simulations. A higher order FE may be used to address this problem, but with increased computation costs. Even if damage were allowed to occur up to the maximum possible value of 1.0, a similar, but delayed, damage pattern may be expected.

(a)

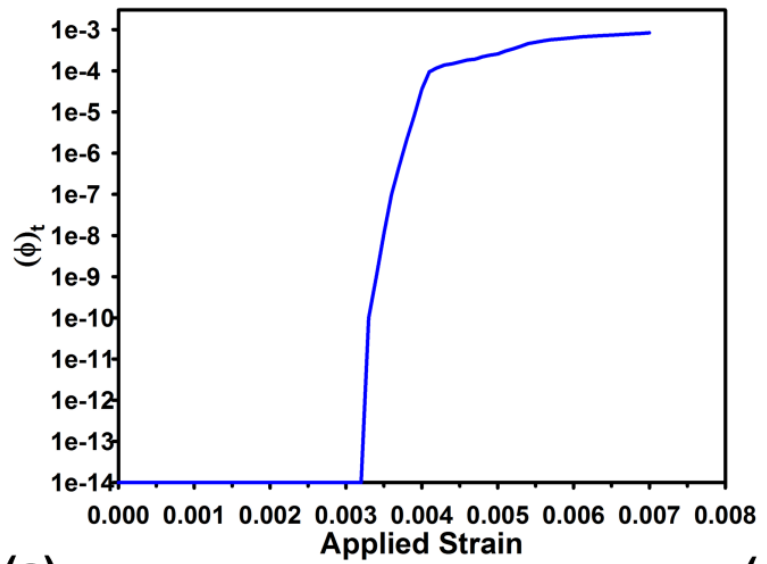

(c)

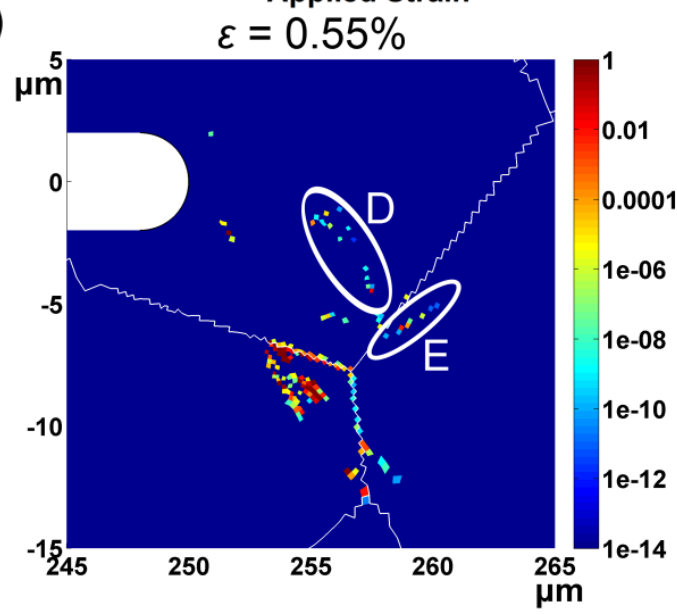

(b)

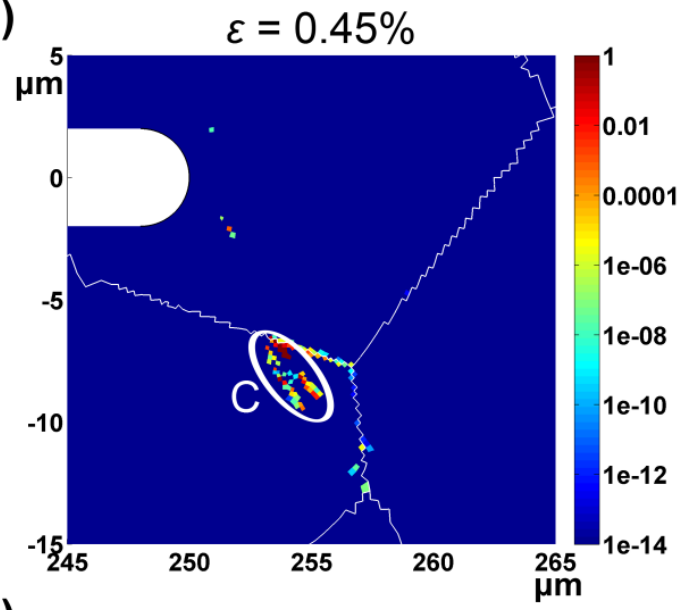

(d)

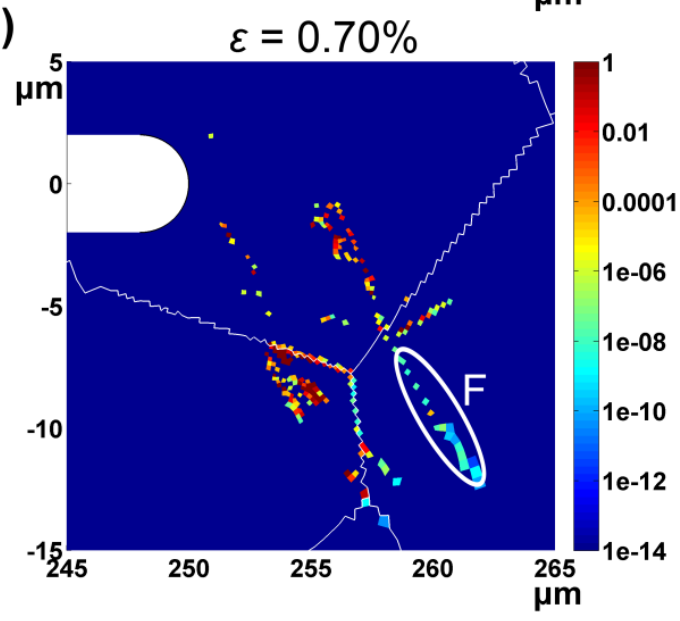

Figure 24. (a): Evolution of total area-weighted damage (in a semi-circular region of radius $25 \mu \mathrm{m}$ ahead of the notch) with applied strain. (b), (c), (d): Distribution of damage in the material after (b) $0.45 \%$, (c) $0.55 \%$, and (d) $0.70 \%$ applied strain, respectively. See text for discussion of regions $\mathrm{C}, \mathrm{D}, \mathrm{E}$ and $\mathrm{F}$. 


\subsubsection{Parametric study of $\Delta \Upsilon_{h e t}$}

Figure 25 (a) shows the evolution of $(\phi)_{t}$ with applied strain for three different values of $\Delta \Upsilon_{\text {het }}$. The material was irradiated to $2.9 \mathrm{dpa}$ and then loaded at $550 \mathrm{~K}$. A notch of $\rho=0.5 \mu \mathrm{m}$ and $a=300 \mu \mathrm{m}$ was used in all these simulations. Further, the same initial microstructure was used for all cases. Note that the simulation for $\Delta \Upsilon_{h e t}=0.32 \mathrm{Jm}^{-2}$ failed to converge beyond $0.2 \%$ applied strain.

As expected, damage initiates earlier with a lower value of $\Delta \Upsilon_{h e t}$. Inspection of the deformed microstructure (not shown here) suggests that the damage initiation patterns are similar in all three cases, albeit delayed in time with increasing $\Delta \Upsilon_{h e t}$. A lower value of $\Delta \Upsilon_{h e t}$ allows failure to initiate prematurely at GBs (provided the hydrostatic stress is sufficient for void nucleation). This is because strain localization within a dislocation channel needs to be high enough such that the difference in $\varepsilon_{\text {eff }}^{i}$ with the neighboring element exceeds 0.05 , for an 'interface' to be created in our simulations (cf. Section 4.2). In the early stages of localization this might not be the case, allowing (aphysical) premature void nucleation at GBs for lower values of $\Delta \Upsilon_{h e t}$.

(a)

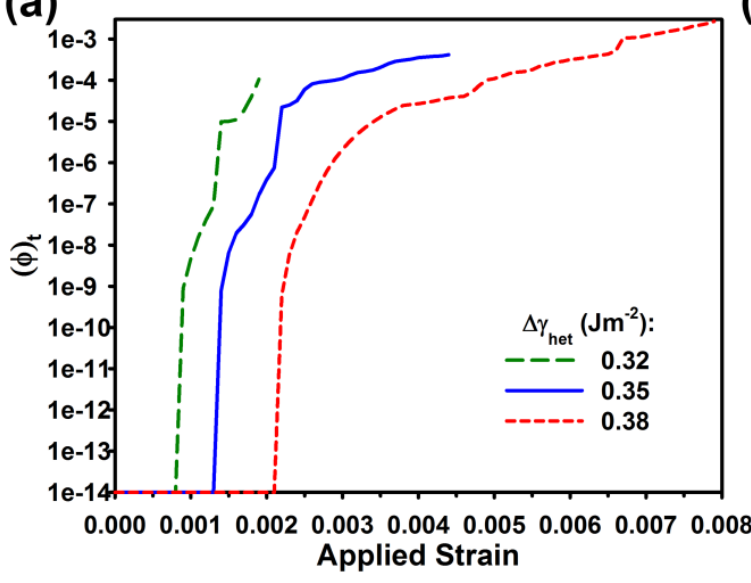

(b)

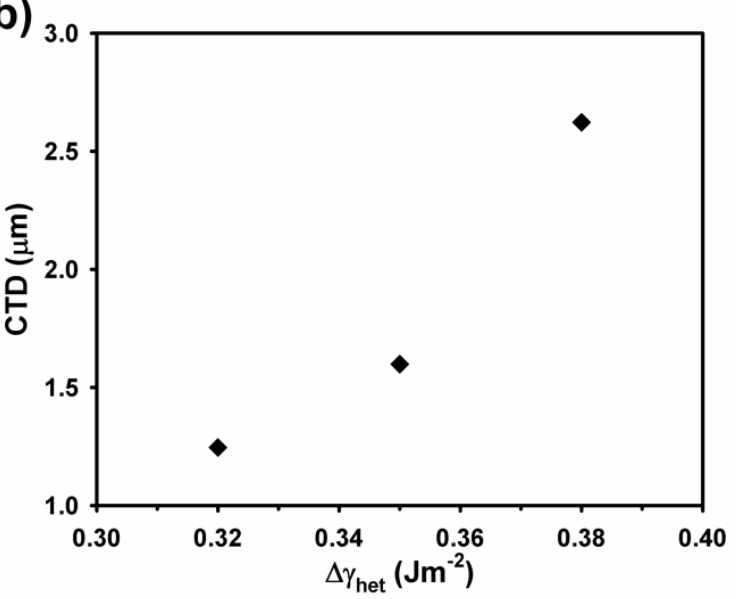

Figure 25. (a): Evolution of total area-weighted damage with applied strain for different values of $\Delta \Upsilon_{h e t}$ (b): Computed $C T D$ as a function of $\Delta \Upsilon_{h e t}$ at $\left.(\phi)_{t}\right|_{c}=1.0 \times 10^{-4}$.

$\Delta \Upsilon_{h e t}$ may be used as a parameter to calibrate our simulations to experiments. For example, if the critical value of damage were to be pre-defined as $\left.(\phi)_{t}\right|_{c}=1.0 \times 10^{-4}$ (say), the 
$C T D$ (or fracture toughness) corresponding to $\left.(\phi)_{t}\right|_{c}$ can be computed for different values of $\Delta \Upsilon_{\text {het }}$ and compared to experiments. Figure 6(b) shows the computed $C T D$ for different $\Delta \Upsilon_{\text {het }}$ at $\left.(\phi)_{t}\right|_{c}=1.0 \times 10^{-4}$. As $\Delta \Upsilon_{h e t}$ increases, $\left.(\phi)_{t}\right|_{c}$ is found to occur at higher CTD. The CTD corresponding to $\Delta \mathrm{\Upsilon}_{\text {het }}=0.38 \mathrm{Jm}^{-2}$ is found to occur closest to the point of intersection of the $5 \%$ secant line (from the origin with linear elastic loading slope) with the load-displacement curve. Experimentally, intersection point of the 5\% secant line with the load-displacement curve is generally used to estimate the critical fracture toughness (cf. [161]). Using $\Delta \Upsilon_{h e t}=0.32 \mathrm{Jm}^{-2}$ or $0.35 \mathrm{Jm}^{-2}$, the computed $C T D$ was found to be much lower. Hence, a value of $\Delta \Upsilon_{h e t}=0.38 \mathrm{Jm}^{-2}$ is used for the rest of our simulations.

It can be seen from the damage evolution plots in Figs. 24 (a) and 25 (a) that $\left.(\phi)_{t}\right|_{c}=1.0 \times 10^{-4}$ roughly indicates the point at which 'stable' damage propagation begins. As will be shown in Section 4.5.7, this value is in agreement with the critical damage corresponding to the computed fracture toughness.

\subsubsection{Crack growth resistance}

The framework developed here is used to estimate the crack growth resistance ( $\mathrm{R}$ curve) in terms of the $C T D$ of a stationary crack (sharp notch) of increasing crack length for monotonic remote loading. A circular notch of $\rho=0.5 \mu \mathrm{m}$ was placed at increasing depths in the range 50$400 \mu \mathrm{m}$ within the material. The dimensions of the crystal plasticity zone, loading and boundary conditions remain the same as shown in Fig. 21 (a). The material was loaded to $0.5 \%$ remote applied engineering strain at a quasi-static strain rate of $10^{-4} \mathrm{~s}^{-1}$ at $550 \mathrm{~K}$. The material response was found to be just at or beyond the point of intersection of the 5\% secant line with the loaddisplacement curve at $0.5 \%$ engineering strain, in all cases. Further, damage initiation was observed in all the irradiated microstructures.

Figure 26 shows the computed $C T D$ of the 2.9 dpa irradiated specimens with increasing crack length, compared to the simulations of the corresponding CTD for virgin (no irradiation) specimens. As expected, a monotonic, R curve behavior is seen for both the irradiated and virgin specimens. Further, the crack growth resistance of the irradiated material is lower than that of the corresponding virgin material in all cases. This agrees with experimental observations regarding the effect of irradiation on crack growth resistance under quasi-static loading for another ferritic/martensitic steel [109], suggesting a loss in tearing resistance with increasing radiation dose. 
A comparison of the deformed microstructures (loaded to $0.5 \%$ remote applied engineering strain) for the $2.9 \mathrm{dpa}$ irradiated and virgin specimens for $a=200 \mu \mathrm{m}$ is shown in Fig. 27. Figures 27 (a) and (c) show the distribution of inelastic strain for the irradiated and the virgin material, respectively. The corresponding damage distribution is shown in Figs. 27 (b) and (d), respectively. The same grain distribution and initial crystallography was used in both cases. As expected, no dislocation channels are observed in the virgin specimen; the inelastic strain localization is typical of a plane strain, small-scale yielding (cf. [161,199]). Further, no damage is observed in the virgin specimens. Physically, failure may initiate in the regions of stress concentration at second phase particles and inclusions (microstructural features not considered here) in the unirradiated specimens. This may lead to even higher CTDs than computed for the virgin specimens in our simulations. In the context of the present constitutive equations, a lower value of $\Delta \Upsilon_{h e t}$ may be used to initiate failure at GB interfaces in the virgin specimens.

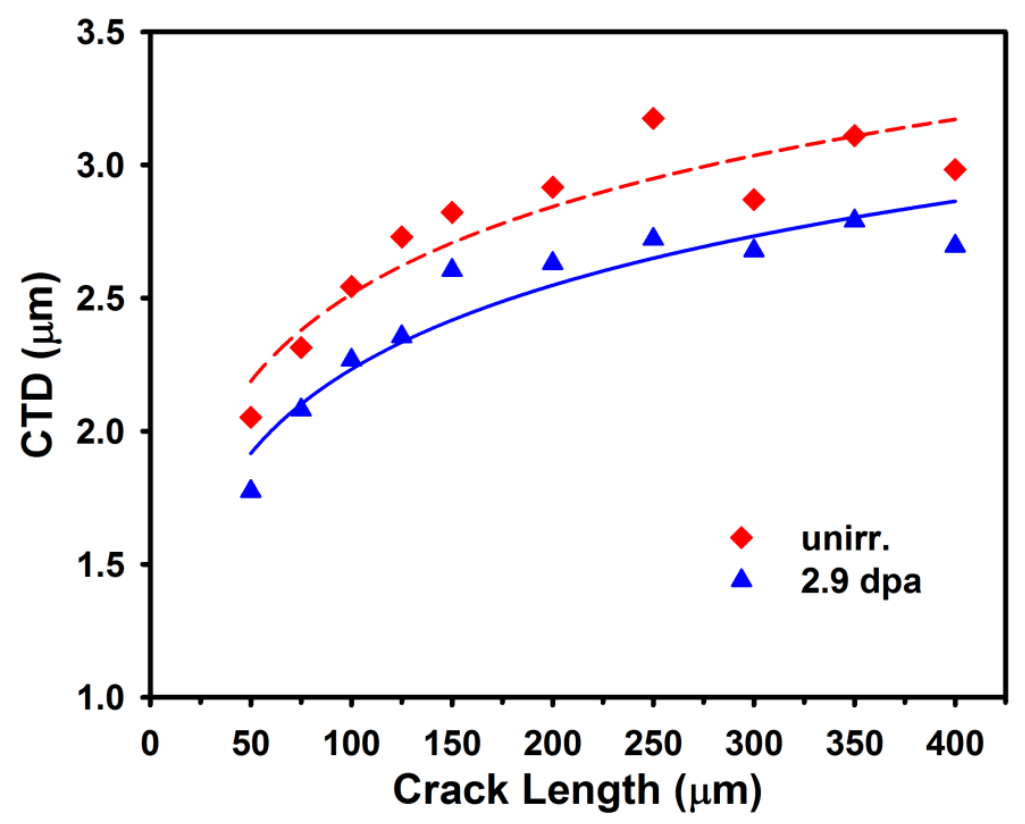

Figure 26. Crack growth resistance of irradiated material compared to that of the virgin material, computed in terms of the $C T D$ of stationary cracks of increasing lengths.

The irradiated microstructure, in contrast, shows failure initiation along interfaces, both dislocation channels and GBs, in the vicinity of the notch. These heterogeneous interfaces provide paths for 'easy' crack propagation in the irradiated material, thus degrading the crack growth resistance. Though not supported by explicit experimental results, Chaouadi [109] suggested a similar mechanism to be responsible for degradation in the crack growth resistance of irradiated materials. FE simulations of void growth at bi-material interfaces [200] have shown 
that void growth is more prolific at the interfaces as compared to that in a homogeneous material. This further asserts that the experimentally observed degradation in crack growth resistance of irradiated specimens is due to 'accelerated' void nucleation and growth processes at the dislocation channel interfaces, as modeled in our simulations. Note that the stress levels ahead of the notch are higher in the irradiated material (higher flow stress due to irradiation hardening) than the virgin material. Higher stress promotes void nucleation according to the constitutive relations given in Eqs. (4.9)-(4.13), all other conditions remaining the same.

(a)

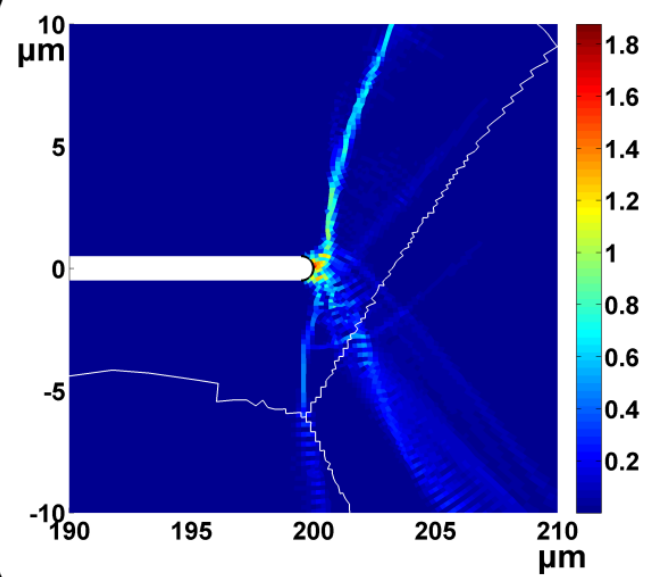

(c)

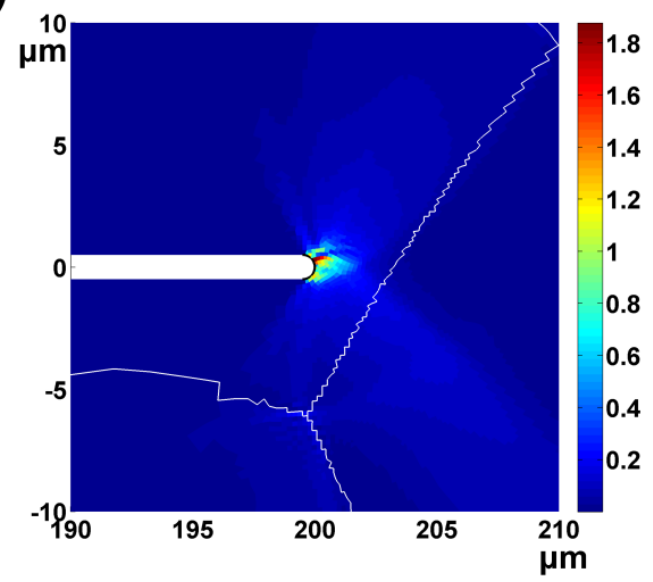

(b)

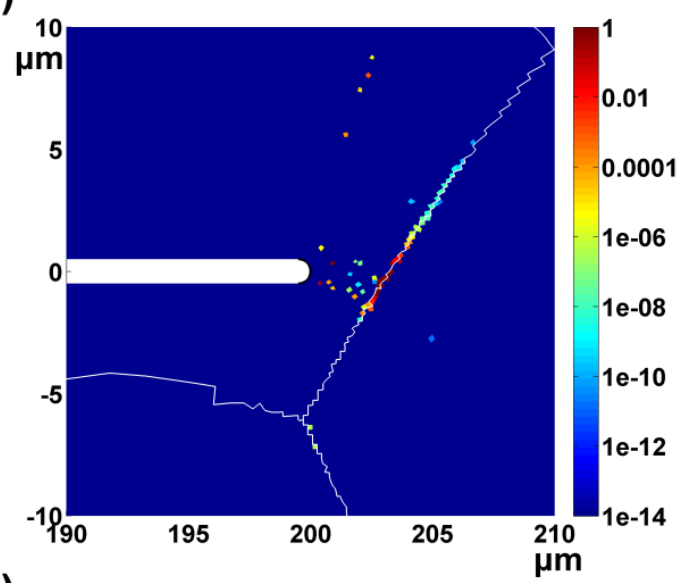

(d)

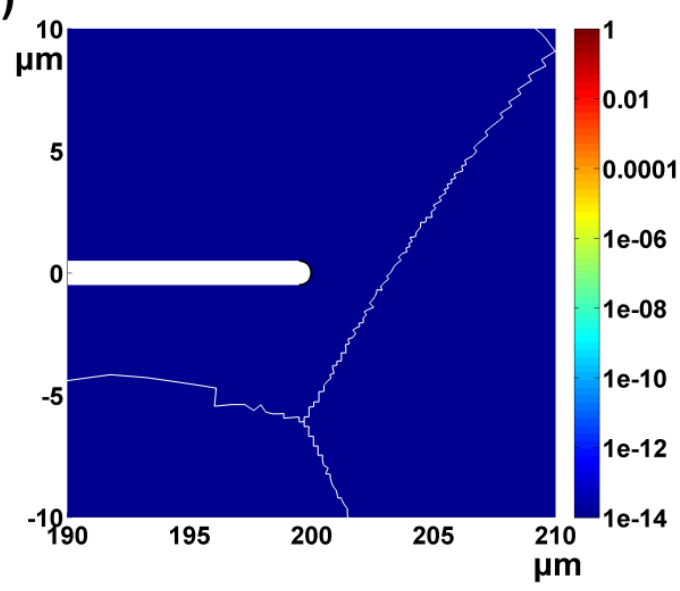

Figure 27. Distribution of effective inelastic strain for (a): irradiated (to 2.9 dpa ), and (c): virgin specimens loaded to $0.5 \%$ strain at $550 \mathrm{~K}$. (b), (d): Distribution of damage corresponding to the microstructures in (a), and (c), respectively.

\subsubsection{Strain rate effects}

Figure 28 (a) shows the inelastic strain distribution ahead of a $300 \mu \mathrm{m}$ deep notch loaded at a strain rate of $1.0 \mathrm{~s}^{-1}$. The same microstructure loaded at a strain rate of $10^{-4} \mathrm{~s}^{-1}$ is shown in Figure 28 (b). The material was irradiated to $2.9 \mathrm{dpa}$ and then deformed to $0.49 \%$ strain at $550 \mathrm{~K}$ 
in both cases. It can be seen that the level of localization is lower at higher strain rate and the dislocation channels are not fully developed. In contrast, parts of the quasi-statically loaded microstructure are already in the post-localization, softening regime. Note that the material is modeled using a viscoplastic flow rule (cf. Eq. (2.4)). Rate effects in limiting 'viscous' inelastic dissipation may lower the extent of localization in the material loaded at $1.0 \mathrm{~s}^{-1}$. We have also verified from the stress-strain curves that the material loaded at higher strain rate has a higher hardening rate (with respect to inelastic strain). It has been shown earlier [201] that localization is accommodated less readily at higher hardening rates. As a result, formation of dislocation channel 'interfaces' and subsequent failure initiation is delayed at higher strain rates. Further, void nucleation and growth, as modeled here, are diffusion-controlled processes. Diffusion time scales are much lower than the time scales of inelastic deformation, especially for the material loaded at $1.0 \mathrm{~s}^{-1}$; fewer voids would nucleate and grow (i.e., less damage) over the time scale of inelastic deformation at this loading rate. From simulations, $\phi_{t}$ was found to be $1.54 \times 10^{-6}$ for the material loaded at $1.0 \mathrm{~s}^{-1}$, and $1.02 \times 10^{-4}$ for the quasi-statically loaded material, after $0.49 \%$ applied strain. This may be used to interpret the experimentally observed increase in crack growth resistance of the irradiated material under dynamic loading [109]. Since the material loaded at higher strain rate has lower damage, its tearing resistance would be higher.

(a)

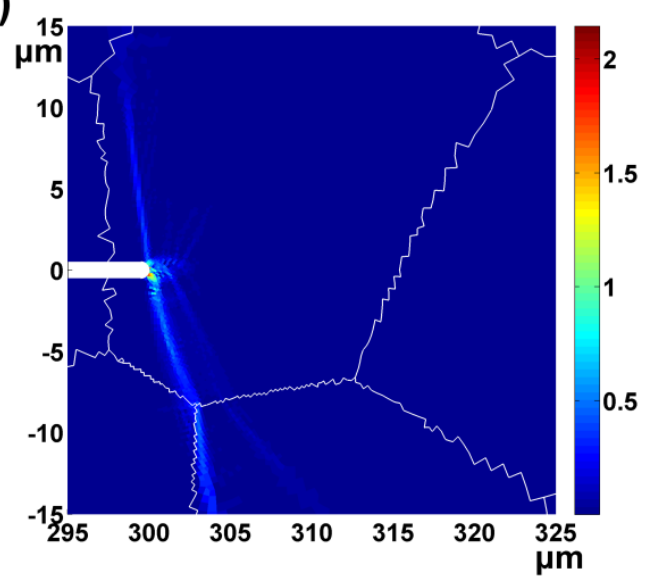

(b)

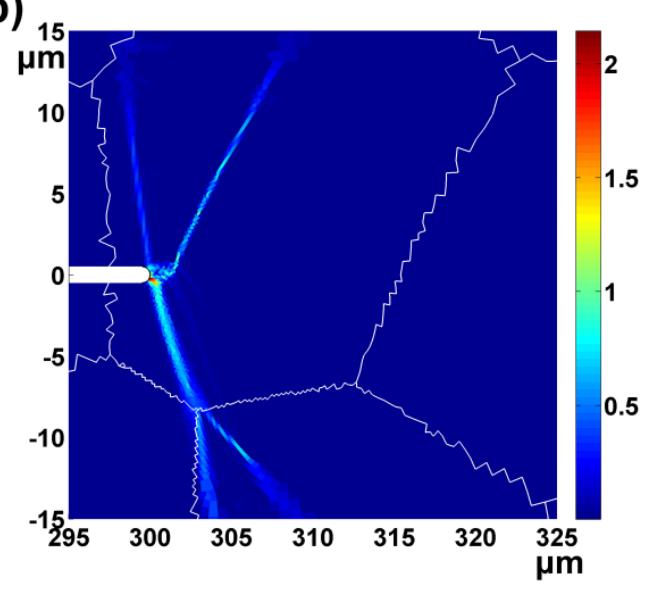

Figure 28. Comparison of inelastic strain distribution for irradiated ( $2.9 \mathrm{dpa}$ ) specimen loaded at a strain rate of (a): $1.0 \mathrm{~s}^{-1}$, and (b): $10^{-4} \mathrm{~s}^{-1}$, to $0.49 \%$ strain at $550 \mathrm{~K}$.

\subsubsection{Temperature effects}

Figure 29 (a) shows the evolution of total area-weighted void number density, $\left(N_{V}\right)_{t}$, for three different test temperatures. The corresponding evolution of total area-weighted void radius, 
$\left(r_{V}\right)_{t}$, is shown in Fig. 29 (b). $\rho=2 \mu \mathrm{m}$ and $a=250 \mu \mathrm{m}$ in all these simulations. The same initial microstructure as in Figs. 22 and 23 was used for all three cases. The material was loaded in tension, after irradiation to $2.9 \mathrm{dpa} .\left(N_{V}\right)_{t}$ and $\left(r_{V}\right)_{t}$ were computed using a similar procedure described for $(\phi)_{t}$ in Section 4.5.2. Note that the simulation failed to converge beyond $0.5 \%$ strain for the $600 \mathrm{~K}$ case.

As can be seen in Fig. 29 (a), voids nucleate earlier in the material loaded at higher temperatures. However, the evolution of $\left(N_{V}\right)_{t}$ is not intuitive beyond the point of failure initiation. The rate of evolution of void number density is higher at higher temperatures due higher thermal 'activation' of void nucleation, as modeled in Eq. (4.10). Inspection of the deformed microstructure (not shown here) revealed roughly the same failure patterns in all three cases, albeit at lower applied strains for the materials loaded at higher temperatures. It can also be seen that $\left(N_{V}\right)_{t}$ for the material loaded at $437 \mathrm{~K}$ is initially lower, but exceeds that for material loaded at $550 \mathrm{~K}$ after $\approx 0.55 \%$ applied strain. This is because the material at lower temperature has relatively higher flow stress, resulting in lower activation energy for void nucleation (cf. Eq. (4.13)). Another reason for this is also due to the fact that the void radius of the material loaded at $437 \mathrm{~K}$ is much lower than that at $550 \mathrm{~K}$ (Fig. 29 (b)), meaning less overall damage. In order to accommodate the incompatible deformation, failure initiation takes place via higher void nucleation rate at $437 \mathrm{~K}$.

(a)

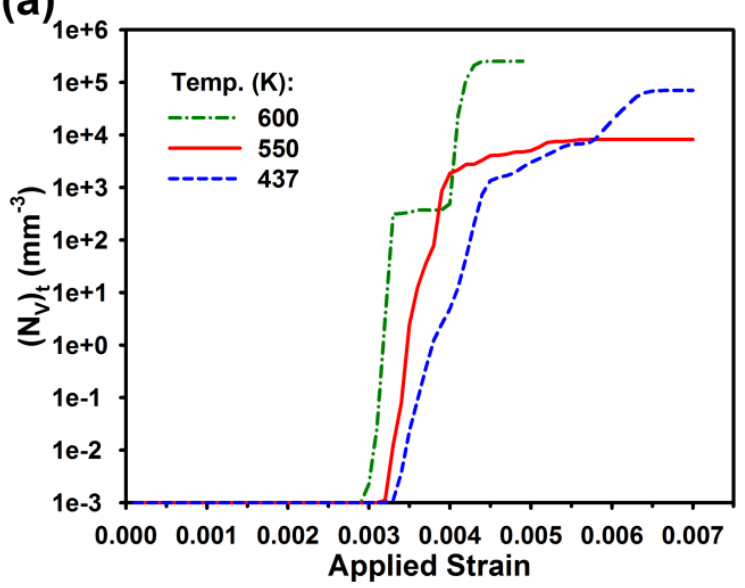

(b)

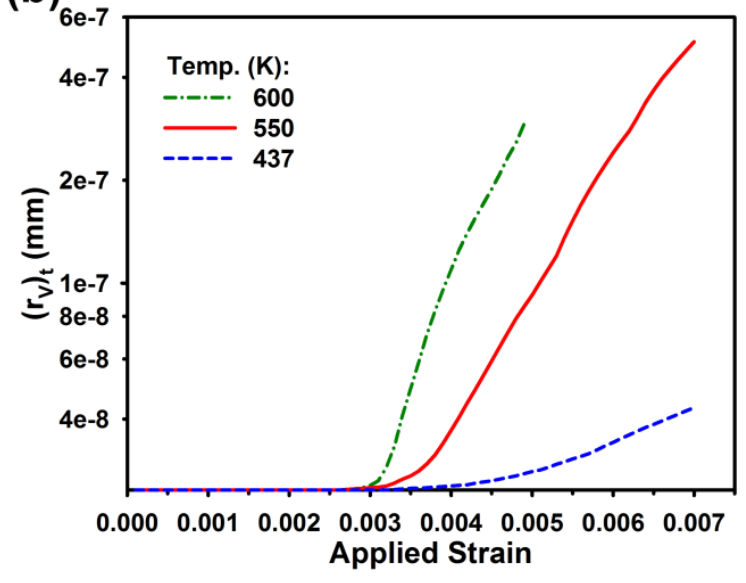

Figure 29. Evolution of (a): total area-weighted void number density, and (b): total areaweighted void radius as a function of temperature.

Figure 29 (b) shows that the evolution of void radius is near-monotonic at all temperatures; the rate of change of void radius is higher at higher temperatures. This is because 
void growth, as modeled here, is governed solely by thermally-activated diffusion kinetics. Void nucleation, on the other hand, is dependent both on the material stress levels and the loading temperature. The void radius is much lower at $437 \mathrm{~K}$ than the higher loading temperatures, indicating a change in failure mechanism from ductile 'zigzag' fracture [109] to limited ductility 'channel' fracture [153] in the temperature range 437-550 K. Note that $\Delta \Upsilon_{h e t}$ was assumed to be temperature invariant in these simulations. However, $\Delta \Upsilon_{h e t}$ may be expected to increase with decrease in temperature, further lowering the rate of void nucleation and growth.

\subsubsection{Fracture toughness calculations}

In the limit of small-scale yielding, the stress intensity factor, $K$, in terms of the $C T D$, is given as [161]

$$
K=\sqrt{m \sigma_{Y S}(C T D) E^{\prime}}
$$

where $m$ is a dimensionless constant dependent on the stress state ( $m=2$ for plane strain), $\sigma_{Y S}$

is the yield stress, and $E^{\prime}=E /\left(1-v^{2}\right)$ for plane strain. As mentioned earlier, the intersection of the $5 \%$ secant line with the load-displacement curve is generally used to assess the critical fracture toughness, $K_{c}$, in experiments. We adopt a similar procedure in the present work, computing the CTD at the point of intersection of the 5\% secant line with the load-displacement curve, and calculating $K_{c}$ using Eq. (4.27). Based on the true stress-true strain curve of Mod 9Cr-1Mo steel irradiated to $2.9 \mathrm{dpa}$ (not presented here), $\left.\sigma_{Y S}\right|_{550 \mathrm{~K}} \approx 725 \mathrm{MPa}$ at $T=550 \mathrm{~K}$. Similarly, $\left.\sigma_{Y S}\right|_{437 \mathrm{~K}} \approx 750 \mathrm{MPa}$, and $\left.\sigma_{Y S}\right|_{600 \mathrm{~K}} \approx 710 \mathrm{MPa}$.

The values of $K_{c}$ calculated for different notch depths and radii are listed in Table 4. There is a large discrepancy in the measured value of $K_{c}$ between simulations and experiments. However, note that the experiments used a much larger notch radius, $\rho$. In general, the measured $K_{c}$ is known to decrease with decreasing $\rho$ for both metals [202-204] and ceramics [205-207]. The same was observed in our simulations, when $\rho$ was decreased from $2 \mu \mathrm{m}$ to 0.5 $\mu \mathrm{m}$. It has also been suggested that $K_{c}$ scales linearly with $\rho^{1 / 2}$, beyond a threshold notch root radius [203,206]. See Refs. [208,209] for analytical model to explain this effect. As shown in Fig. 30, if we were to extrapolate the simulated $K_{c}$ as a function of $\rho^{1 / 2}$ for $a / W=0.5$, the predicted $K_{c}$ at $\rho=60-80 \mu \mathrm{m}$ is consistent with the experimental values. Possible reasons of slightly lower values of $K_{c}$ reported in experiments may be due to higher radiation doses and lower test temperatures (reflecting in lower ductility). Also note that the experimental notch root 
radii are larger than the grain size $(\approx 25 \mu \mathrm{m})$. At these notch root radii, intergranular interactions may homogenize and mitigate the effect of grain level heterogeneity on the fracture toughness that are observed for sub-grain size notch root radii, for example in the present simulations. Further, fracture toughness has also been found to vary with irradiation temperature [160]. These effects are beyond the scope of the present work and not considered here.

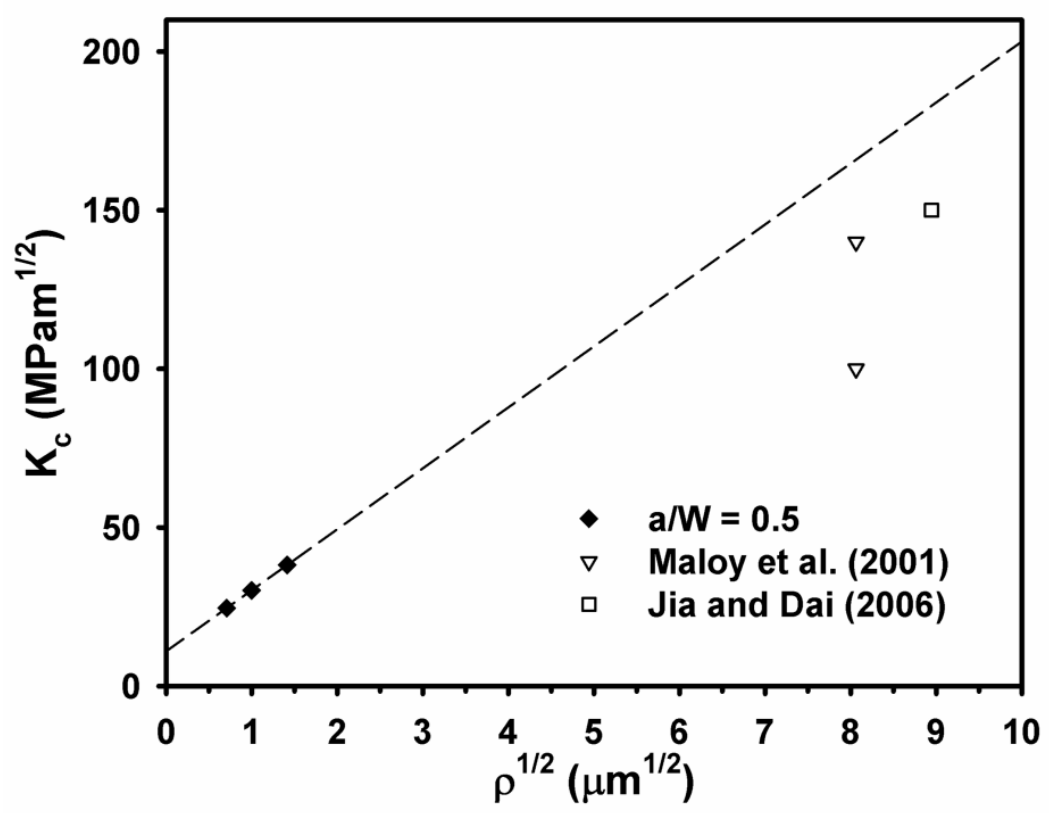

Figure 30. $K_{c}$, as a function of $\rho^{1 / 2}$, extrapolated to the experimental notch radii. Experimental data from [2,3].

It can be seen from Table 4 that the value of $(\phi)_{t}$ at critical fracture toughness is in the range $10^{-5}-10^{-4}$ at $550 \mathrm{~K}$. We have shown earlier in Section 4.5.3 that this value roughly corresponds to the stable damage propagation regime. In terms of classical fracture mechanics, the driving force for fracture, $K$, overcomes the material resistance, $K_{c}$, at this critical $(\phi)_{t}$ to allow stable crack propagation. Further, $(\phi)_{t}$ decreases with decrease in temperature, indicating a decrease in ductility. $(\phi)_{t}$ may thus be used as an indicator of the material's fracture behavior in the present framework. 
Table 4. Fracture toughness calculations for different notch radii, $\rho$, and notch depths, $a$. $W=800 \mu \mathrm{m}$ is the width of the specimens.

\begin{tabular}{lllllll}
\hline $\begin{array}{l}\text { Radiation } \\
\text { dose }(\text { dpa })\end{array}$ & $T(K)$ & $\rho(\mu \mathrm{m})$ & $a / W$ & $(\phi)_{t}$ & $C T D(\mu \mathrm{m})$ & $K_{c}(\mathrm{MPa} \sqrt{\mathrm{m}})$ \\
\hline 2.9 & 550 & 0.5 & 0.3125 & $2.44 \times 10^{-5}$ & 2.35 & 26.61 \\
2.9 & 550 & 0.5 & 0.375 & $2.11 \times 10^{-5}$ & 2.21 & 25.84 \\
2.9 & 550 & 0.5 & 0.4375 & $1.03 \times 10^{-4}$ & 2.00 & 24.58 \\
2.9 & 550 & 0.5 & 0.5 & $3.29 \times 10^{-5}$ & 2.01 & 24.60 \\
2.9 & 550 & 1.0 & 0.5 & $1.05 \times 10^{-5}$ & 3.01 & 30.15 \\
2.9 & 550 & 2.0 & 0.5 & $1.86 \times 10^{-5}$ & 4.83 & 38.18 \\
2.9 & 550 & 1.0 & 0.3125 & $1.97 \times 10^{-4}$ & 3.27 & 31.41 \\
2.9 & 550 & 2.0 & 0.3125 & $1.48 \times 10^{-4}$ & 5.41 & 40.40 \\
2.9 & 437 & 2.0 & 0.3125 & $1.1 \times 10^{-9}$ & 5.12 & 40.74 \\
2.9 & 600 & 2.0 & 0.3125 & $4.18 \times 10^{-4}$ & 5.50 & 39.97 \\
3.0 & $323-433$ & $\sim 65$ & 0.5 & - & - & $100-140^{1}$ \\
4.3 & 523 & $\sim 80$ & 0.5 & - & - & $\approx 150^{2}$ \\
\hline
\end{tabular}

${ }^{1}$ Ref. [2], ${ }^{2}$ Ref. [3].

\subsection{Summary}

A void nucleation and growth based continuum damage framework has been developed and coupled with an existing crystal plasticity framework, to model failure initiation in irradiated bcc materials. Constitutive equations for vacancy generation via cross-slip and dislocation dipole mechanisms (during inelastic deformation) have been developed. Void nucleation has been modeled using a classical nucleation theory, while void growth is modeled using a diffusioncontrolled mechanism. The framework has been used to model vacancy supersaturation along dislocation channels, and void nucleation and growth along these dislocation channel interfaces and GBs at high stress triaxialities. Our simulations have been able to capture the microstructural evolution characteristics of the transgranular 'zigzag' failure mechanism [109] at intermediate 
temperatures. Further, the crack growth resistance of the irradiated notched specimens has been found to be lower than that of the corresponding virgin specimens. The extent of localization and damage (in the irradiated microstructures) was found to decrease with increase in the loading rate. The effect of temperature on the evolution of void number density and radius has also been studied. There is a discrepancy between the irradiated specimen fracture toughness measured from simulations and from experiments. However, when the simulated fracture toughness values are extrapolated to the experimental notch root radii, they are consistent with the values measured from experiments.

The simulated failure initiation is strongly sensitive to the value of the interfacial energy change, $\Delta \Upsilon_{h e t}$, for heterogeneous void nucleation at dislocation channel interfaces and GBs. As has been demonstrated, this parameter may be used to calibrate the simulations to experimental results, in terms of the measured crack tip displacement and/or critical fracture toughness. Insights from atomistic simulations of the interfacial phenomena may also be used to refine the estimation of this parameter, for example its temperature dependence. Further, the value of $\Delta \mathrm{\Upsilon}_{\text {het }}$ adopted here is used to study failure initiation ahead of sharp notches having radii in the range 0.5-2 $\mu \mathrm{m}$. At lower stress triaxialities (larger notch root radii), this value of $\Delta \Upsilon_{h e t}$ may be too high to allow any void nucleation. Future work will address these issues by performing detailed parametric studies of the effect of notch geometry on failure initiation. Future work can also incorporate strain-driven void growth mechanisms in this framework, to model failure over larger applied strains.

Defect-tolerant design of structures used in nuclear applications often relies on the fracture toughness master curve of the material used (cf. [210]). This framework (calibrated to experiments) may be used to construct such master curves for various loading histories. Further, the framework developed here is pretty general and may be applied to simulate void nucleation and growth-mediated failure for other deformation histories (no irradiation) as well, for example, at slip band interfaces during cyclic loading, or at second phase interfaces, etc. in different material systems. 


\section{CHAPTER 5}

\section{MODELING IRRADIATION CREEP DEFORMATION}

\subsection{Introduction}

Irradiation creep deformation of metallic systems is accelerated (in comparison to thermal creep) due to the creation of point defects that promote the growth and migration of defect loops and voids by stress-assisted mechanisms. Further, irradiation creep occurs at relatively lower homologous temperatures [7]. Irradiation creep has been correlated to the volumetric changes and swelling observed in structural metals [33,190]. While ferritic/martensitic steels (materials modeled in this work) are generally known to have good swelling resistance at elevated temperatures [10,11], irradiation creep would still contribute to the creation and growth of defect loops. Subsequent glide of dislocations through the field of these irradiation-induced defect loops would be impeded.

This Chapter focuses on modeling the mechanical behavior and microstructure evolution during irradiation creep deformation. The crystal plasticity framework developed in Chapter 2 is modified to account for absorption of radiation-induced point defects at grain boundary (GB) sinks. The framework is used to simulate the substructure in terms of dislocation densities, interstitial loop densities, and point defect concentrations. The effect of radiation rate, applied stress, and grain size on the creep behavior is studied. Parametric study of the GB sink model is also performed. The creep strain is also correlated to the in-situ radiation damage (measured in dpa).

\subsection{Background}

The microstructure of metals undergoing irradiation creep deformation is characterized by the formation of defect loops, dislocation pileups and subgrain boundaries [211,212]. The evolution of loops is initially prolific, with saturation reached at higher dpa doses [213]. In order to account for the stress anisotropy, the evolution of strain and defects was correlated [211] to the stress induced preferential absorption (SIPA) model $[28,38]$. Again, the SIPA model was not able to account for the entire deformation all by itself [213]. As mentioned in Section 2.2.2, glide of dislocations subsequent to their climb (by SIPA) also needs to be accounted for. Further, it was found [211] that a threshold radiation dose exists after which irradiation creep commences. The creep strain rate (with respect to dpa rate) has also been found to be a near-linear function of the applied stress [214]. Studies also suggest [215] that a transition stress exists beyond which the creep exponent changes from unity to a higher value. 
A review of the irradiation creep mechanisms was given in Section 2.2.2. Without going into the details, it is again emphasized that SIPA and preferential absorption glide (PAG) of dislocations are the dominant mechanisms contributing to irradiation creep, at least in the steady state regime. We have modeled the same using the climb-enhanced glide mechanism in Section 2.3.3.2.3.

The constitutive model developed in Chapter 2 did not account for the absorption of radiation-induced point defects at GBs. While this assumption may be valid for certain cases of pure metals and single crystals, point defect-GB interactions go hand-in-hand with the radiationinduced solute segregation effects observed in alloys [6]. For example, irradiation has been found to cause depletion of $\mathrm{Cr}$, and enrichment of $\mathrm{Ni}$ and $\mathrm{Si}$ at the GBs in stainless steels [216]. Since atomic transport is generally accompanied by a parallel point defect transport (interstitial or vacancy, depending on the direction), the fluxes of these solute atoms are often related to the point defect fluxes [6]. We do not model the effects of radiation on solute segregation explicitly, but we have considered point defect absorption at GBs in this Chapter and studied its effect on the propagation of inelastic deformation across GBs during creep loading.

\subsection{Model description}

The finite deformation crystal plasticity framework developed in Chapter 2 models the following ISVs: mobile dislocation density, $\rho_{M}^{\alpha}$, immobile dislocation density, $\rho_{I}^{\alpha}$, interstitial loop density, $N_{i}^{\alpha}$, interstitial loop size, $d_{i}^{\alpha}$, at the level of slip system $\alpha$, and the interstitial, $c_{i}$, and vacancy concentration, $c_{v}$, at the macroscopic level.

Constitutive equations for the evolution of slip system level ISVs are defined in Chapter 2, while those for the point defect evolution are modified here to account for absorption at GB sinks. Specifically, Eqs. (2.9) and (2.10) representing the rate of evolution of interstitials, $\dot{c}_{i}$, and vacancies, $\dot{c}_{v}$, are modified to the following form:

$$
\begin{gathered}
\dot{c}_{i}=K_{0}-K_{i v} c_{i} c_{v}-\sum_{\alpha} K_{i s}^{\alpha}\left(\rho_{M}^{\alpha}+\rho_{I}^{\alpha}\right) c_{i}-k_{i G B}^{2} D_{i} c_{i} \\
\dot{c}_{v}=K_{0}-K_{i v} c_{i} c_{v}-\sum_{\alpha} K_{v s}^{\alpha}\left(\rho_{M}^{\alpha}+\rho_{I}^{\alpha}\right) c_{v}-k_{v G B}^{2} D_{v} c_{v}
\end{gathered}
$$

where $K_{0}, K_{i v}$ are the defect pair creation and recombination rates, respectively, $K_{i s}^{\alpha}=\left|z_{i}^{\alpha}\right| D_{i}$ is the interstitial-dislocation reaction rate coefficient, $K_{v s}^{\alpha}=\left|z_{v}^{\alpha}\right| D_{v}$ is the vacancy-dislocation reaction rate coefficient, and $k_{i G B}, k_{v G B}$ are the GB sink strengths for interstitials and vacancies, respectively, and $D_{i}, D_{v}$ are the interstitial and vacancy diffusivities, respectively. Here, $z_{x}^{\alpha}=z_{x}^{0}\left(\sigma_{c}^{\alpha} / E\right)$ is the point defect capture efficiency of defect type $x, \sigma_{c}^{\alpha}=\boldsymbol{n}^{\alpha} \cdot \boldsymbol{\sigma} \cdot \boldsymbol{n}^{\alpha}$ is the 
resolved stress normal to the slip plane $\alpha$ with unit normal direction, $\boldsymbol{n}^{\alpha}, E$ is the Young's modulus, and $z_{x}^{0}$ is a material constant. While the rest of the terms were defined earlier in Chapter 2, the sink strength, $k_{x G B}$, represents the tendency of the GB to absorb defect, $x$ [6,217]. Physically, $k_{x G B}^{-1}$ represents the distance travelled in the GB by the defect $x$ before it gets absorbed [6].

In the present work, we use a spatially resolved mesh to solve for the point defect rate equations and also couple them with the crystallography-dependent deformation behavior. Note that in our model implementation, $k_{x G B}=0$ when the material point is not at a GB. This is in contrast with coarse-grained mean field rate theory calculations (e.g. $[63,83]$ ), where an average value of GB sink strength is assumed. While these are useful for studying the time evolution of ISVs and associated properties, spatial correlation is beyond the scope of the mean field rate theory calculations.

It is again emphasized that we have neglected the full character of transport relations with defect gradients and fluxes in the present formulation. Physically, point defect fluxes would exist from the grain interior to the GBs, more so in the presence of radiation-induced segregation. In the presence of defect gradients, the point defect evolution equations will have the following form:

$$
\begin{gathered}
\dot{c}_{i}=K_{0}-K_{i v} c_{i} c_{v}-\sum_{\alpha} K_{i s}^{\alpha}\left(\rho_{M}^{\alpha}+\rho_{I}^{\alpha}\right) c_{i}-k_{i G B}^{2} D_{i} c_{i}+\nabla \cdot\left(D_{i} \nabla c_{i}\right) \\
\dot{c}_{v}=K_{0}-K_{i v} c_{i} c_{v}-\sum_{\alpha} K_{v s}^{\alpha}\left(\rho_{M}^{\alpha}+\rho_{I}^{\alpha}\right) c_{v}-k_{v G B}^{2} D_{v} c_{v}+\nabla \cdot\left(D_{v} \nabla c_{v}\right)
\end{gathered}
$$

where the last term in each equation represents the respective diffusive flux due to spatial concentration gradients. Incorporating these defect gradients requires non-local continuum modeling. We leave this to future work.

\subsection{Model implementation}

\subsubsection{Loading and boundary conditions}

The 3D constitutive model was implemented in the finite element program ABAQUS [86] using the User MATerial (UMAT) subroutine. Generalized plane strain finite elements were used along with 3D material crystallography. Grains with in-plane square cross-section were used, thus effectively simulating a network of cuboidal grains. Random initial crystallographic orientations were assigned to all the grains. Finite elements with linear interpolation and reduced integration were used.

Creep tests with concurrent irradiation were simulated on the polycrystalline ensembles. Figure 31 shows a schematic of the loading and boundary conditions. The bottom edge of the 2D 
simulation box is constrained along the loading direction, and the center of the bottom edge constrained in all degrees of freedom to prevent rigid body rotation. The side edges are tractionfree. Stress-controlled loading is applied to the top edge. The applied tensile stress is ramped up to the desired level at an effective strain rate of $10^{-4} \mathrm{~s}^{-1}$, and then the stress is held constant for the desired amount of time.

This model differentiates between a GB and grain interior; elements with first nearest neighbors belonging to a different grain are assigned as GB elements.

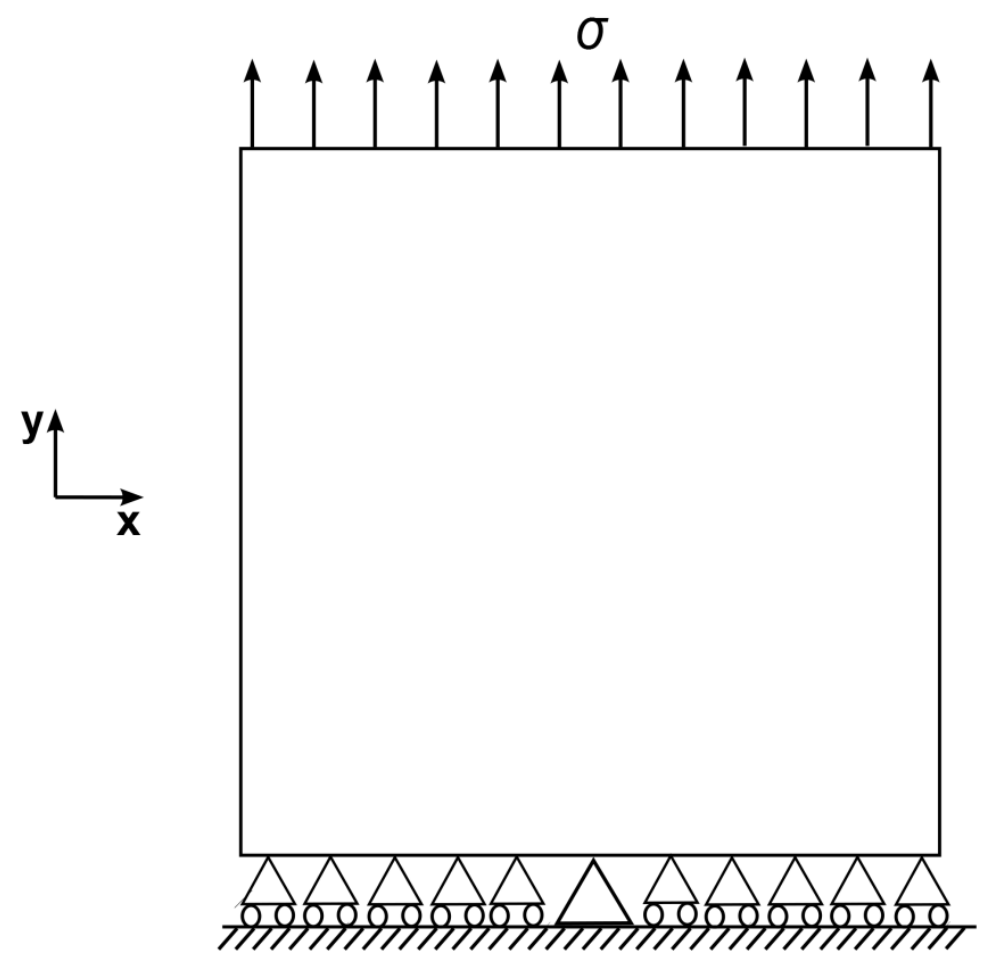

Figure 31. Schematic of stress-controlled loading and boundary conditions.

\subsubsection{Model parameters}

The constitutive model was used to simulate the creep response of Mod 9Cr-1Mo ferritic/martensitic steel. A uniform bcc crystal structure with 48 slip systems is assumed. It is assumed that the dislocation population is entirely mobile at the beginning of the simulation with an initial dislocation density of $\rho_{M}^{0}=1.0 \times 10^{8} \mathrm{~mm}^{-2}$ on each slip system. This dislocation density is of the same order as that observed in experiments [88,89]. It is assumed that there are no immobile dislocations and interstitial loops present initially. Further, an initial concentration of $c_{i}^{0}=c_{v}^{0}=1.0 \times 10^{10} \mathrm{~mm}^{-3}$ was used for both the interstitials and vacancies. As explained in Chapter 2, though the initial point defect concentrations may be expected to be lower in the 
actual material, the high irradiation rate (in terms of $K_{0}$ ) ensures that the specified initial concentrations would be reached momentarily subsequent to the start of irradiation in physical experiments.

Model parameters for the constitutive framework are given in Section 2.4.1. The GB absorption model adds two new parameters, $k_{i G B}$ and $k_{v G B}$, to the framework. It was suggested [217] that $k_{x G B}^{2} \approx 10^{9} \mathrm{~mm}^{-2}$ for a mean grain size $>10 \mu \mathrm{m}$ and defect type $x$. Unless otherwise specified, we have used a value of $k_{x G B}^{2}=5.0 \times 10^{9} \mathrm{~mm}^{-2}$ for both interstitial and vacancy type defects. We have also performed a parametric study of the effect of GB sink strength on creep strain evolution in Section 5.4.3.

\subsection{Results and discussion}

Creep tests with concurrent irradiation were performed on 49 square-shaped, randomly oriented, $25 \mu \mathrm{m}$-sized grains, unless specified otherwise. An initial stress hold was applied for 3 $\mathrm{h}$ before the start of irradiation (point defect generation) in all cases. This allows the applied stress to equilibrate before irradiation-induced deformation begins. The material was loaded at $773 \mathrm{~K}$ in all cases. A mesh size of $2.5 \mu \mathrm{m}$ was used in these simulations, thus having 10 elements along the length of each grain. The following Sections will first consider the simulated mechanical response of the material, and then discuss the microstructure evolution in terms of the effective inelastic strain, dislocation and interstitial loop densities, and interstitial concentrations.

\subsubsection{Mechanical behavior}

Figure 32 (a) shows the evolution of strain as a function of time for different radiation rates (in terms of the defect pair generation rate, $K_{0}$ ) at an applied stress of $160 \mathrm{MPa}$. The corresponding instantaneous strain rates are plotted in Fig. 32 (b). The same initial (random) crystallographic orientation distribution was used in all cases. The strain rate goes up with increase in $K_{0}$; higher $K_{0}$ promotes faster generation of point defect pairs (according to Eqs. (5.1), (5.2)), and corresponding higher dislocation climb rate (Eq. (2.6)) and interstitial loop creation rate (Eq. (2.11)). At a total strain of 0.0016 (near steady-state), the instantaneous strain rate is: $2.69 \times 10^{-9} \mathrm{~s}^{-1}$ for $K_{0}=1.0 \times 10^{12} \mathrm{~mm}^{-3} \mathrm{~s}^{-1}, 5.34 \times 10^{-9} \mathrm{~s}^{-1}$ for $K_{0}=2.0 \times 10^{12} \mathrm{~mm}^{-3} \mathrm{~s}^{-1}$, $2.23 \times 10^{-8} \mathrm{~s}^{-1}$ for $K_{0}=5.0 \times 10^{12} \mathrm{~mm}^{-3} \mathrm{~s}^{-1}$, and $3.27 \times 10^{-8}$ for $K_{0}=10.0 \times 10^{12} \mathrm{~mm}^{-3} \mathrm{~s}^{-1}$. Based on this data, the instantaneous strain rate was found to scale almost linearly as a function of $K_{0}$. 
(a)

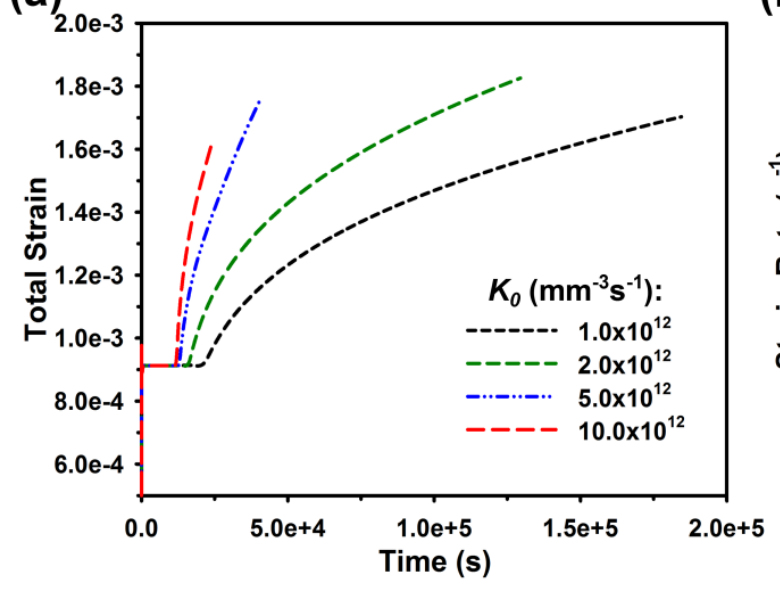

(b)

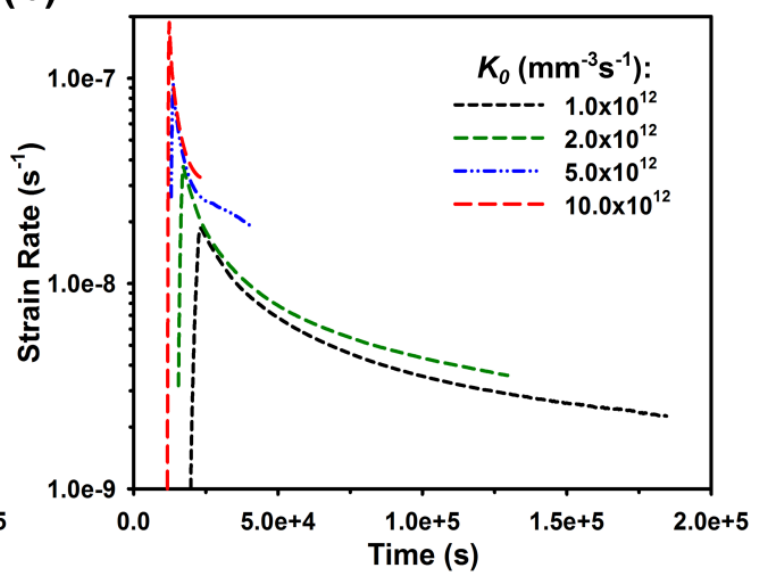

Figure 32. (a): Evolution of total strain as a function of time for different $K_{0}$, (b): evolution of instantaneous strain rate corresponding to the strain evolution in (a).

More interestingly, it is observed in Fig. 32 (a) that following the initial stress hold period of $3 \mathrm{~h}$, irradiation creep does not begin at the same instant for the different radiation rates. Irradiation creep begins earlier for the material irradiated at higher $K_{0}$. This suggests that a radiation threshold needs to be reached before irradiation creep commences. Radiation threshold has also been observed in experimental studies of irradiation creep in 304 and 316 stainless steels $[211,218]$ as well.

Correlation with the evolution of average interstitial loop density (Fig. 33 (a)) and loop size (Fig. 33 (b)) suggests that this radiation dose threshold is associated with the initial generation of interstitial loops and saturation of the average interstitial loop size. Note that the evolution rate of loop size, $\dot{d}_{i}^{\alpha}$, depends on the rate of absorption of interstitials by climbing mobile dislocations and is a linear function of the interstitial concentration, $c_{i}$ (cf. Eq. (2.13)). As observed in Fig. 9 in Chapter 2, $c_{i}$ saturates following the initial transients, leading to saturation of $d_{i}^{\alpha}$, as well. It is noted that the loops are expected to grow in size with increasing radiation. Evolution of multiple size classes of loops at a material point may be used to capture this experimentally observed effect in a rate theory model (e.g. [213]). A size class based model allows for transport of point defects from one size class to the next by diffusion-mediated absorption/emission of point defects. However, these calculations are computationally intensive, especially so in the context of the present spatially resolved finite element crystal plasticity framework, where the ISVs have to be resolved at the slip system level, as well.

The interstitial loop density, on the other hand, increases monotonically as a function of time. The creation rate of interstitial loops, $\dot{N}_{i}^{\alpha}$, is proportional to the climb rate, $\dot{\gamma}_{c}^{\alpha}$ (cf. Eq. 
(2.11)), and hence has a similar evolution trend as the creep strain. Deo et al. [50] suggested a square root dependence of the interstitial loop density on the radiation dpa level, i.e.,

$$
N_{i}=A \cdot \mathrm{dpa}^{1 / 2}
$$

where $A=1.0 \times 10^{15} \mathrm{~mm}^{-3}$ for low radiation levels ( $<1 \mathrm{dpa}$ ). While this phenomenological relation was proposed to correlate the 'post-irradiation' interstitial loop density with the dpa level, we have used this relation to measure the in-situ dpa level during the irradiation creep simulations. Given that the physics of defect pair production is beyond the scope of the present continuum framework, this is the only feasible way of measuring radiation dpa levels during our irradiation creep simulations.

(a)

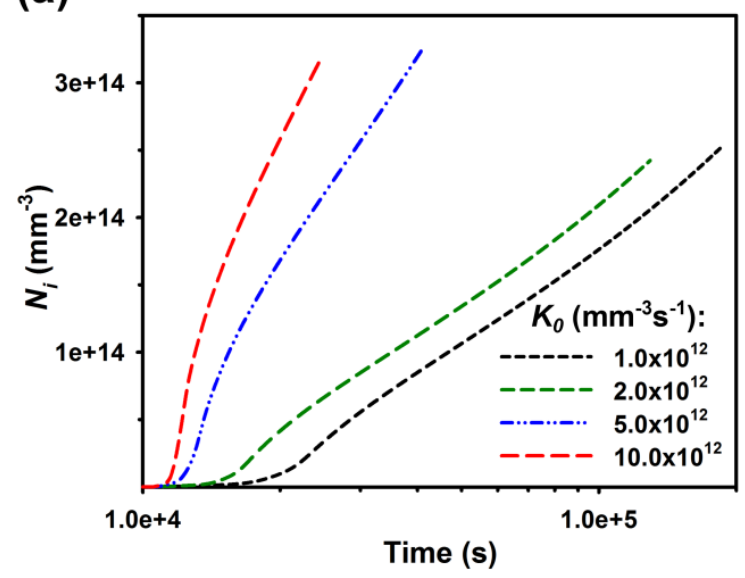

(b)

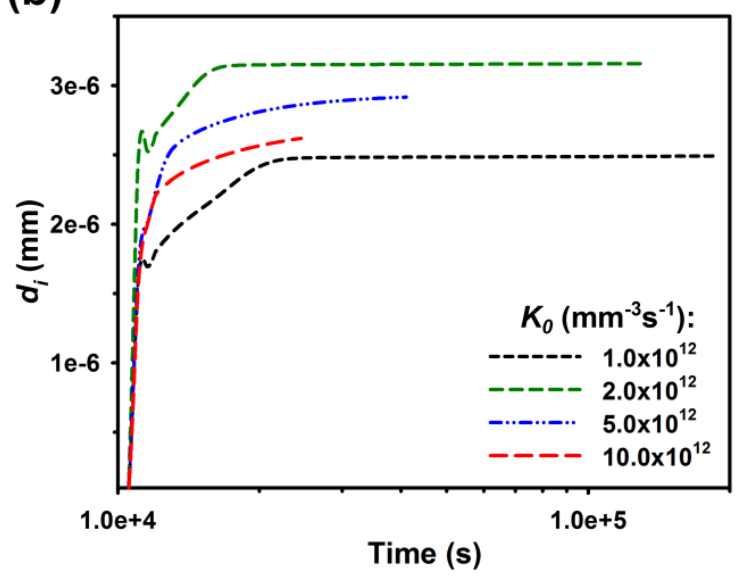

Figure 33. Evolution of (a): average interstitial loop density, and (b): average interstitial loop size as a function of time for different radiation rates.

Figure 34 (a) shows the evolution of radiation dpa level with time. The radiation dpa is found to increase linearly with time for all radiation rates, subsequent to the initial stress hold period. This observation has two implications. First, this suggests that the phenomenological relation in Eq. (5.5) is measuring radiation damage correctly as the dpa level is expected to increase linearly with time for a constant radiation rate. More importantly, this asserts that the constitutive model given in Eq. (2.11) is indeed capturing the physics of interstitial loop creation during irradiation creep, since a linear dpa evolution rate is never prescribed in our simulations, rather it emerges from the simulation measurements. A caveat of estimating radiation dpa using this procedure is that there is no way to measure the initial threshold dpa required for irradiation creep deformation to commence. Given that this threshold dpa is expected to be very small, this should not affect the correlation of steady state irradiation creep with dpa level. Presumably, another problem with measuring radiation damage in this manner is that the dpa damage rate is 
not solely a function of the defect pair production rate, $K_{0}$. The measured dpa damage rate is expected to change if one or more of the other loading conditions and material variables change.

(a)

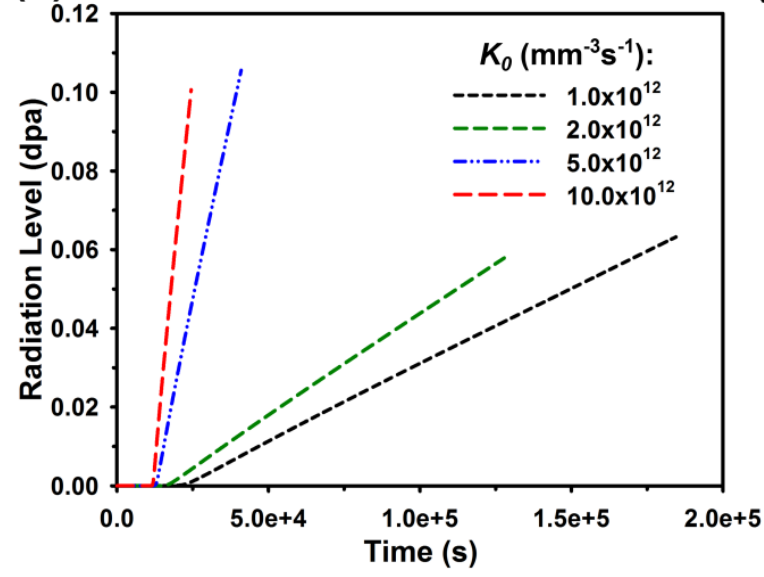

(b)

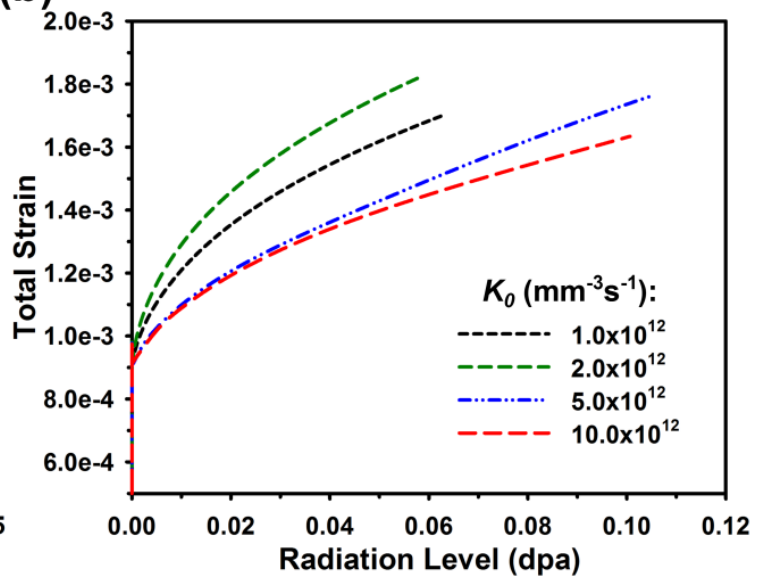

Figure 34. (a): Evolution of radiation dpa level as a function of time using Eq. (5.5), (b): evolution of total strain as a function of radiation dpa level for different $K_{0}$.

In any case, the radiation dpa rate was measured to be: $3.9 \times 10^{-7} \mathrm{dpa} \cdot \mathrm{s}^{-1}$ for $K_{0}=1.0 \times 10^{12} \mathrm{~mm}^{-3} \mathrm{~s}^{-1}, \quad 5.1 \times 10^{-7} \mathrm{dpa} \cdot \mathrm{s}^{-1}$ for $K_{0}=2.0 \times 10^{12} \mathrm{~mm}^{-3} \mathrm{~s}^{-1}, \quad 3.61 \times 10^{-6} \mathrm{dpa} \cdot \mathrm{s}^{-1}$ for $K_{0}=5.0 \times 10^{12} \mathrm{~mm}^{-3} \mathrm{~s}^{-1}$, and $7.94 \times 10^{-6} \mathrm{dpa} \cdot \mathrm{s}^{-1}$ for $K_{0}=10.0 \times 10^{12} \mathrm{~mm}^{-3} \mathrm{~s}^{-1}$ using Eq. (5.5). This suggests that the radiation dpa level is not a linear function of $K_{0}$. Again, the physics of point defect generation due to energetic particle flux is beyond the scope of the present continuum framework. See Was [6] for dependence of the dpa rate on the displacement cross-section, particle flux, energy of the incident particles.

Regardless, the purpose of this exercise is to correlate the evolution of strain (and other properties, as well) to the radiation dpa level during irradiation creep deformation. For example, Fig. 34 (b) shows the evolution of total strain as a function of the average dpa level. The initial strain at zero radiation level indicates the strain accumulated during initial loading of the material to the desired stress. The reason for highest strain rate (w.r.t. dpa) at $K_{0}=2.0 \times 10^{12} \mathrm{~mm}^{-3} \mathrm{~s}^{-1}$ is not evident intuitively. Correlating this to the loop size evolution in Fig. 33 (b), the defect pair generation rate is optimal at $K_{0}=2.0 \times 10^{12} \mathrm{~mm}^{-3} \mathrm{~s}^{-1}$, such that the density of mobile dislocations that have climbed produced the largest loops (in size) by absorbing interstitials. At higher $K_{0}$, greater number of interstitial loops are generated such that less number of interstitials are 
available per interstitial loop formed (cf. Eq. (2.12)). At lower $K_{0}$, the interstitial concentration in the material is lower, and consequently the growth rate of the interstitial loops is also lower.

\subsubsection{Deformed microstructure}

\subsubsection{Spatial distribution of inelastic strain}

Figure 35 shows the distribution of effective inelastic strain in the material loaded at an applied stress of $160 \mathrm{MPa}$ at $773 \mathrm{~K}$. Following the initial stress hold of $3 \mathrm{~h}$, the material was irradiated at a rate of $K_{0}=5.0 \times 10^{12} \mathrm{~mm}^{-3} \mathrm{~s}^{-1}$ for $\approx 10 \mathrm{~h}$. The total strain in the material is $\approx$ 0.00176 .

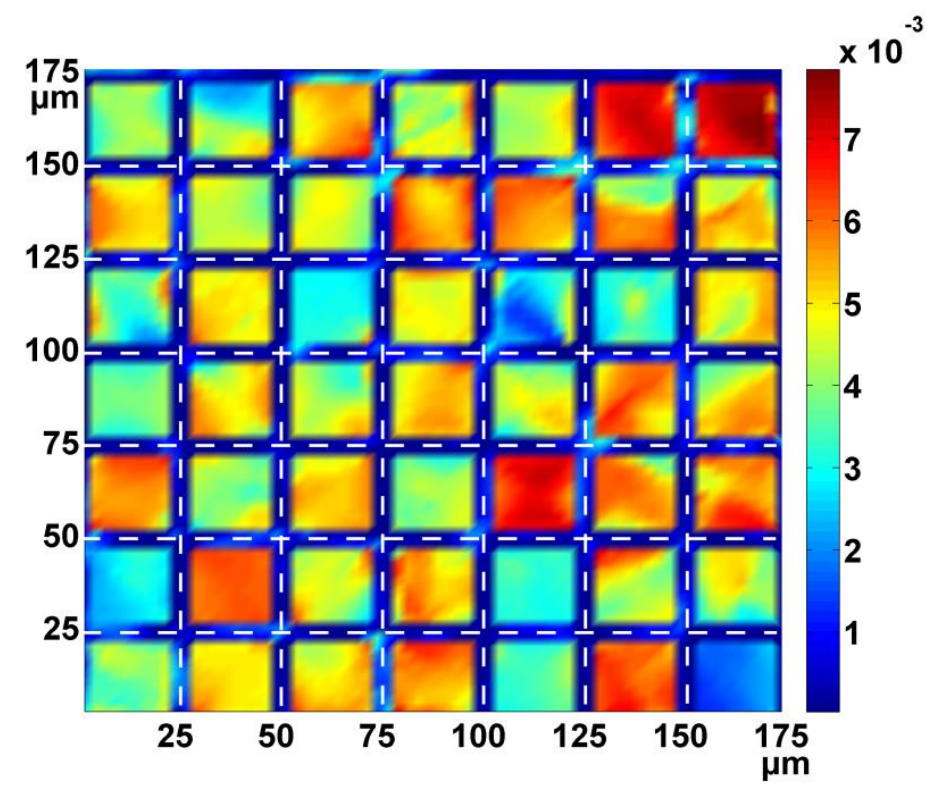

Figure 35. Distribution of inelastic strain in the material loaded at an applied strain of $160 \mathrm{MPa}$ at $773 \mathrm{~K}$. The material was irradiated at $K_{0}=5.0 \times 10^{12} \mathrm{~mm}^{-3} \mathrm{~s}^{-1}$ for $\approx 10 \mathrm{~h}$. Dotted lines overlaid on the mesh indicate GBs.

Depending on the initial crystallographic orientation, the level of deformation varies in each grain interior. However, significantly less deformation is observed at the GBs (regions adjacent to the dotted lines overlaid in Fig. 35). According to the model described in Section 5.3, GBs act as sinks for point defects. Relatively less number of point defects is available for absorption by the deformation-carrying mobile dislocations near GBs. Consequently, creep deformation is limited near the GB regions. In other words, transfer of deformation across GBs is inhibited by the GB sinks. 


\section{$\underline{\text { 5.5.2.2 Substructure }}$}

Figures 36 (a)-(d) show the distribution of substructure in terms of the (slip system averaged) mobile dislocation density, immobile dislocation density, interstitial loop density, and the interstitial concentration, respectively, corresponding to the inelastic strain plot in Fig. 35.

The mobile dislocation density is in general lower in the grain interior as compared to the GB regions. This is because mobile dislocations are absorbed and 'transformed' to interstitial loops in the grain interior, where the interstitial concentration is relatively higher. In contrast, the 'transformation' of mobile dislocations to interstitial loops is limited at the GBs due to the lack of interstitials, thus resulting in higher mobile dislocation density near the GBs.

A part of the mobile dislocation density is also transformed to immobile dislocations via dislocation trapping events (cf. Section 2.3.3.3.3). The rate of mobile dislocation trapping and consequent formation of immobile dislocations is higher in the grain interior, since the deformation is higher. In contrast, the average immobile dislocation density is lower at the GBs. A similar spatial evolution pattern is also observed for the interstitial loop density. This again can be explained by the contrast in deformation and resulting transformation of mobile dislocations to interstitial loops in the grain interior versus the GBs. Spatial evolution of dislocations and interstitial loops is dependent on the availability of interstitials for absorption by mobile dislocations, thus creating interstitial loops. Since GB sinks absorb all the point defects near the grain edges, the interstitial concentration is significantly lower in these regions. The interstitial concentration in the grain interior varies proportional to the amount of deformation within the grains. 
(a)

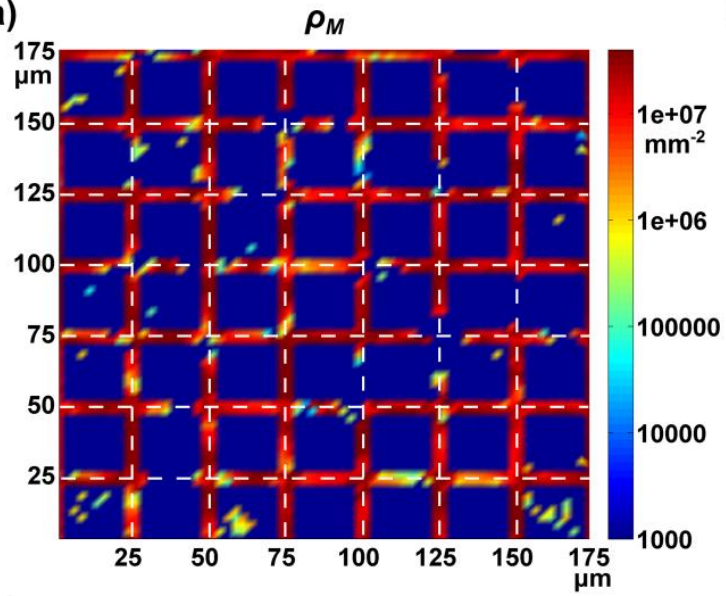

(c)

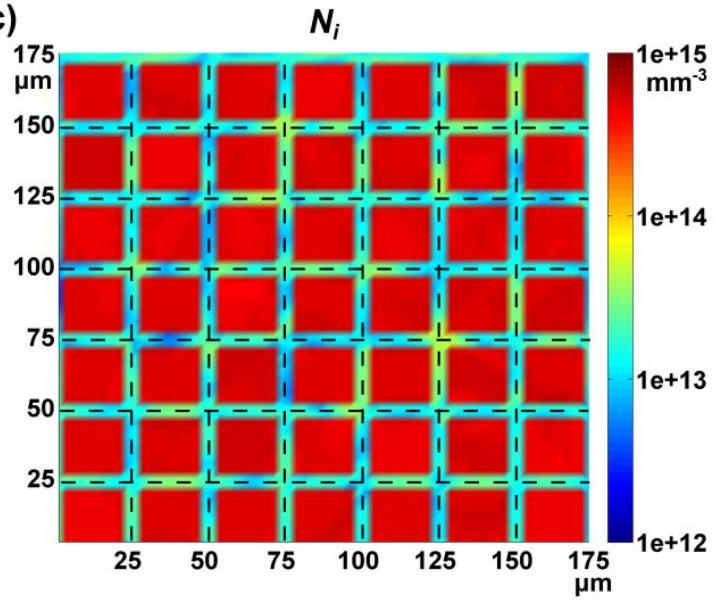

(b)

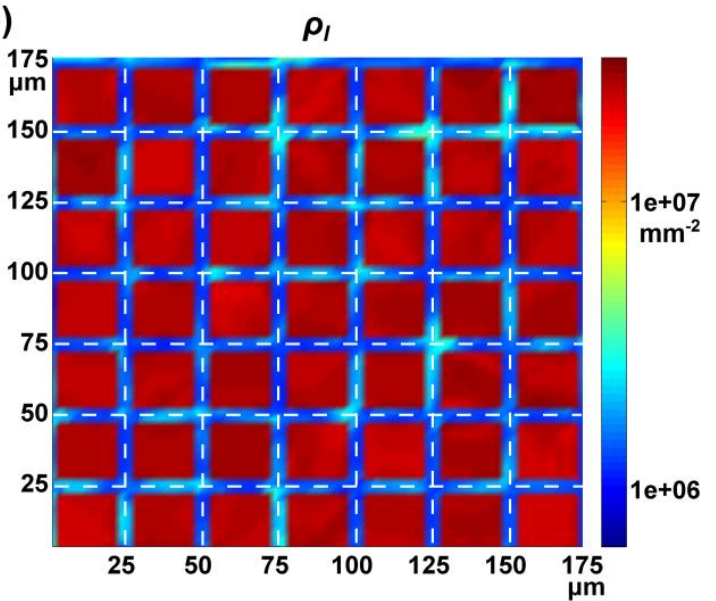

(d)

$c_{i}$

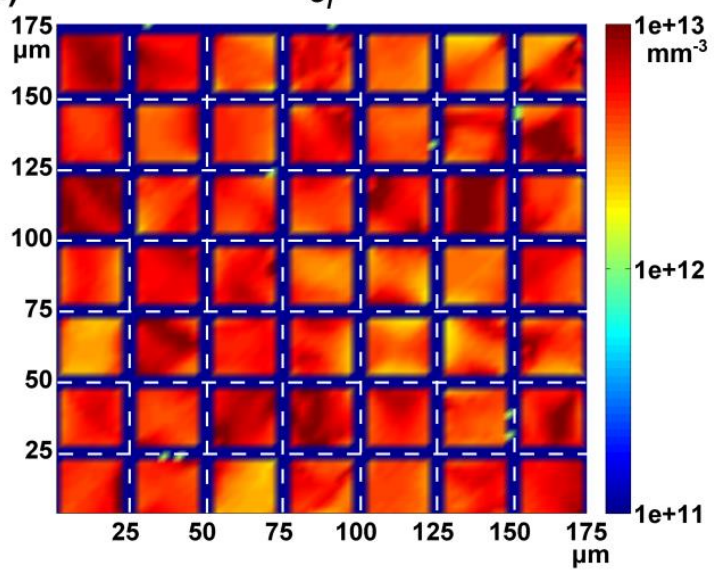

Figure 36. Distribution of (a): mobile dislocation density, (b): immobile dislocation density, (c): interstitial loop density, and (d): interstitial concentration corresponding to the inelastic distribution plot in Fig. 35.

\subsubsection{Parametric study of GB sink model}

We performed a parametric study of the GB sink model by varying the GB sink strengths, $k_{i G B}^{2}$ and $k_{v G B}^{2}$, and studying its effect on the creep strain evolution. The defect pair generation rate was $K_{0}=2.0 \times 10^{12} \mathrm{~mm}^{-3} \mathrm{~s}^{-1}$ in the material loaded at $160 \mathrm{MPa}$ applied stress at $773 \mathrm{~K}$ in all cases. Keeping all other parameters constant, the GB sink strength was varied from $5.0 \times 10^{8} \mathrm{~mm}^{-2}$ to $5.0 \times 10^{10} \mathrm{~mm}^{-2}$. We also considered a case with no GB sinks present, i.e., $k_{i G B}^{2}=k_{v G B}^{2}=0$. Figure 37 (a)-(c) shows the deformed microstructure for three different GB sink strengths at $\approx 0.00157$ total strain. Figure 37 (d) compares the creep strain evolution w.r.t. time for the different sink strengths. It is more intuitive to compare the strain evolution w.r.t. time 
than dpa since the damage rate measured in terms of $\mathrm{dpa} \cdot \mathrm{s}^{-1}$ will not be the same for all cases in our simualtions (different average interstitial loop density evolution for different cases).

The deformed microstructure shows that there is significant deformation at the GBs for the microstructure with zero sinks. As the sink strength increases, the amount of inelastic strain along the GBs decreases. Note that all three microstructures are compared at the same total strain. In order to accommodate the total applied deformation, the degree of deformation in the grain interior is higher for the microstructures with non-zero sink strengths. The strain evolution plot in Fig. 37 (d) shows that the strain rate (w.r.t. time) increases as the GB sink strength decreases and more deformation is transferred across the GBs. Further, the strain evolution is identical for the sink strengths $5.0 \times 10^{9} \mathrm{~mm}^{-2}$ and $5.0 \times 10^{10} \mathrm{~mm}^{-2}$. This suggests that for $K_{0}=2.0 \times 10^{12} \mathrm{~mm}^{-3} \mathrm{~s}^{-1}$, majority of the point defects are absorbed by GBs having sink strengths $5.0 \times 10^{9} \mathrm{~mm}^{-2}$ and higher.

(a)

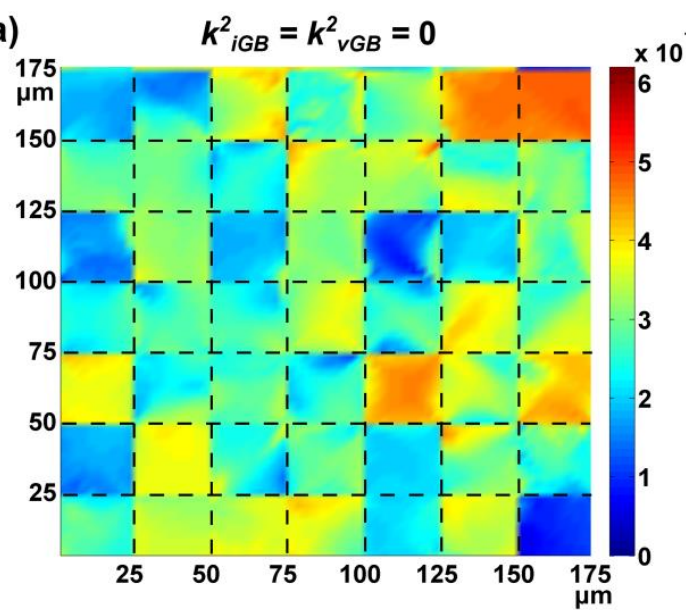

(c) $\quad k^{2}{ }_{i G B}=k^{2}{ }_{v G B}=5.0 \times 10^{9} \mathrm{~mm}^{-2} \times 10^{-3}$

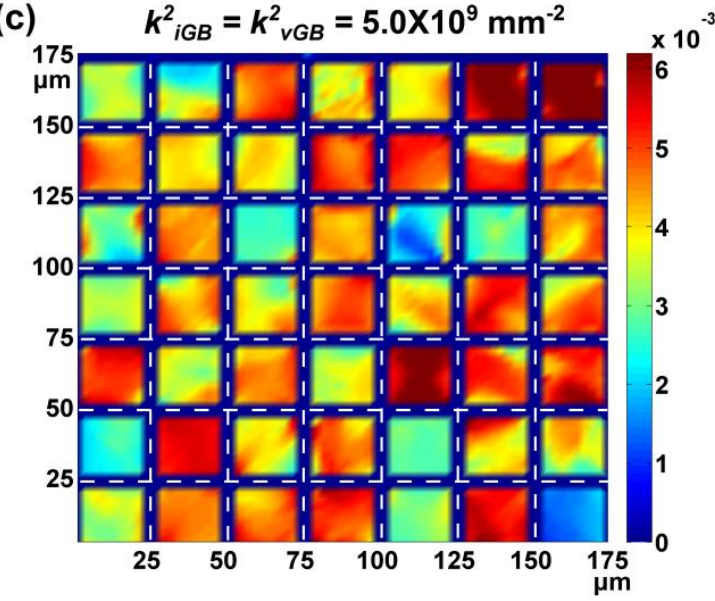

(b)

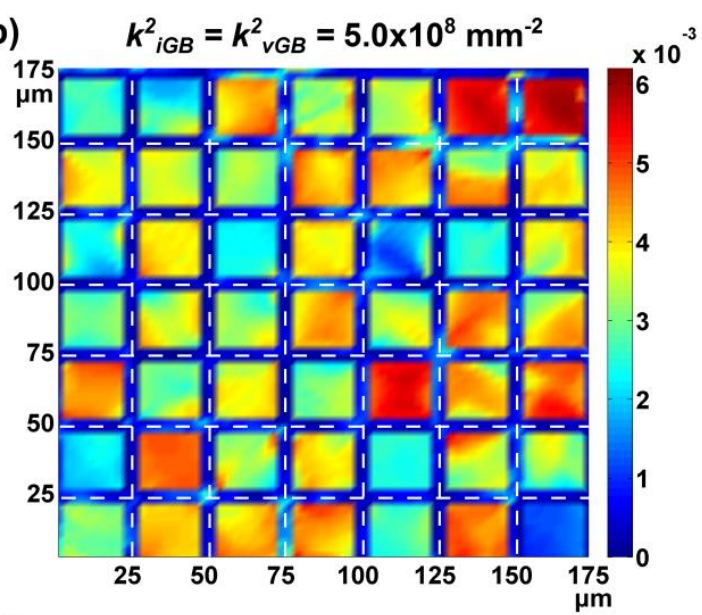

(d)

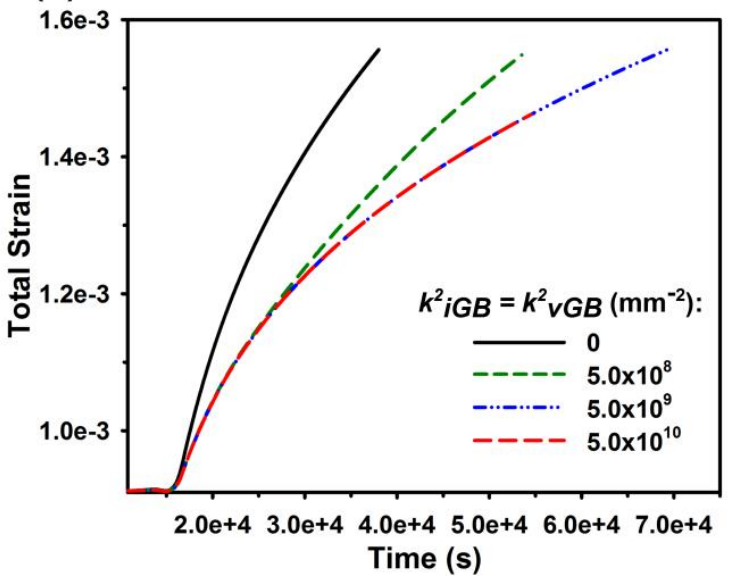

Figure 37. (a-c): Distribution of inelastic strain for three different GB sink strengths. Dotted lines overlaid on the mesh indicate grain interfaces. (d): Evolution of strain as a function of time for different GB sink strengths. 
Since GB sinks lower the irradiation creep rate, it might seem beneficial to engineer materials with higher sink strengths. However, note that we have not accounted for the point defects once they are absorbed at the GBs. Physically, these point defects, especially vacancies, might contribute to intergranular failure via void nucleation and growth processes at the GBs. Further, incompatibility of strain at the GBs might also accelerate these failure processes. As mentioned earlier, point defect absorption at GBs is also associated with radiation-induced segregation (cf. [6]), which is again detrimental for the material's fracture resistance. A model considering all these physical phenomena may be used to estimate the optimal amount of GB sink strength that would prolong the material's life in irradiation environments.

\subsubsection{Grain size effect}

The effect of GB sinks on the creep strain evolution was also studied by varying the density of GBs. Keeping the simulation cell dimensions constant, we performed three separate simulations with 196 grains of $12.5 \mu \mathrm{m}$ size, 49 grains of $25 \mu \mathrm{m}$ size, and 25 grains of $35 \mu \mathrm{m}$ size, respectively. The same element size of $2.5 \mu \mathrm{m}$ was used in all cases. Random initial crystallographic orientation distribution was assigned in all three cases. $K_{0}=5.0 \times 10^{12} \mathrm{~mm}^{-3} \mathrm{~s}^{-1}$ was used. The material was loaded to $\approx 0.0016$ total strain at an applied stress of $160 \mathrm{MPa}$ at 773 K.

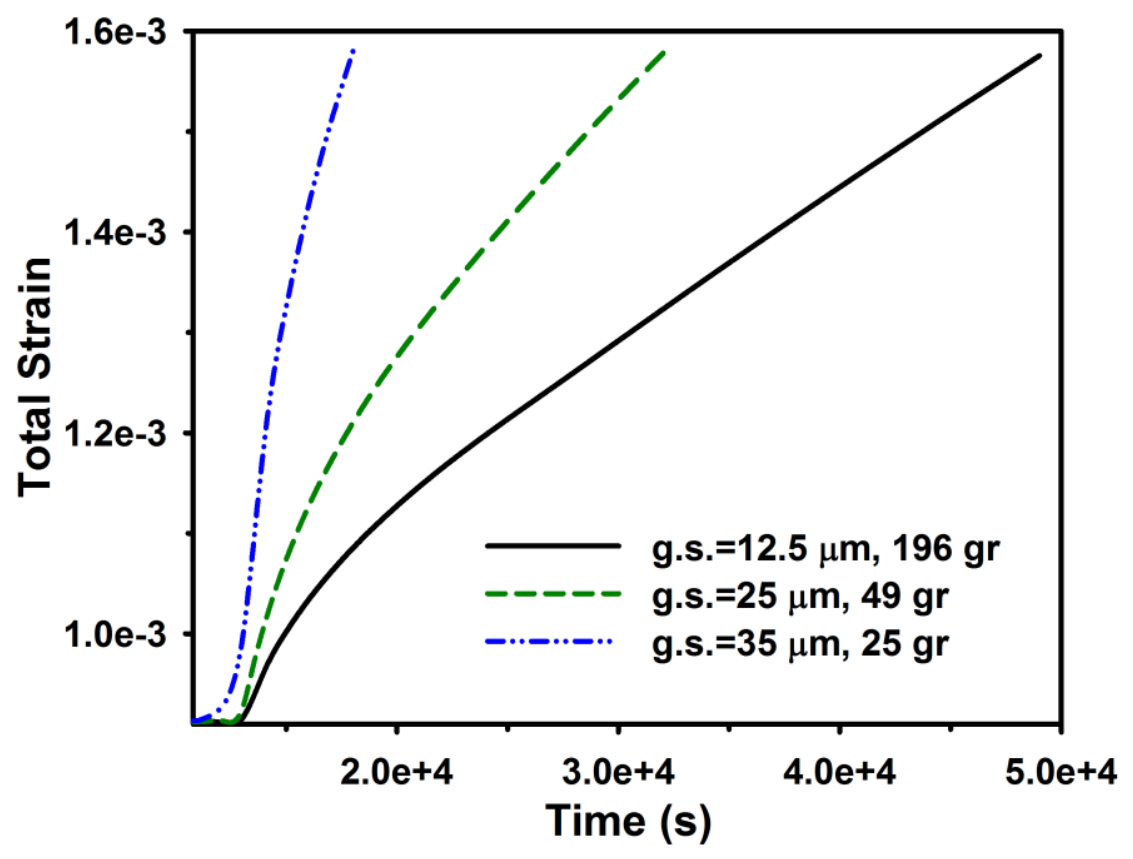

Figure 38. Evolution of creep strain as a function of time for different grain sizes in a simulation cell having the same dimensions. 
Figure 38 shows the evolution of strain (w.r.t. time) for different grain sizes. As the density of GBs increases (i.e., grain size decreases), the creep strain rate decreases. This is because GBs inhibit the transfer of deformation. The grain interior, which is expected to deform at similar rates in all cases, has to be crept longer to achieve the same amount of deformation for the smaller grain sizes (higher density of GBs). Decrease in irradiation creep rate with decreasing grain size was also observed in experiments during in-reactor stress relaxation of $\mathrm{Zr}$ alloys $[219,220]$.

\subsubsection{Irradiation creep compliance}

Assuming a linear stress dependence, the creep rate as a function of stress is given as [221]

$$
\varepsilon^{\prime}=B_{0} \sigma+\varepsilon_{0}^{\prime}
$$

where $\varepsilon^{\prime}$ is the strain rate per dpa rate (measured in $\mathrm{dpa}^{-1}$ ), $B_{0}$ is the creep compliance (measured in $\mathrm{dpa}^{-1} \mathrm{MPa}^{-1}$ ), $\sigma$ is the stress (measured in $\mathrm{MPa}$ ), and $\varepsilon_{0}^{\prime}$ is the strain offset at zero stress (measured in $\mathrm{dpa}^{-1}$ ). Irradiation creep simulations were performed at three different applied stresses $(80 \mathrm{MPa}, 160 \mathrm{MPa}$, and $240 \mathrm{MPa})$ for $K_{0}=2.0 \times 10^{12} \mathrm{~mm}^{-3} \mathrm{~s}^{-1}$ and $K_{0}=5.0 \times 10^{12} \mathrm{~mm}^{-3} \mathrm{~s}^{-1}$. The irradiation temperature was $773 \mathrm{~K}$ in all cases. The in-situ radiation dpa level was calculated using Eq. (5.5) and correlated to the total strain. $\varepsilon^{\prime}$ was measured in the steady-state creep regime.

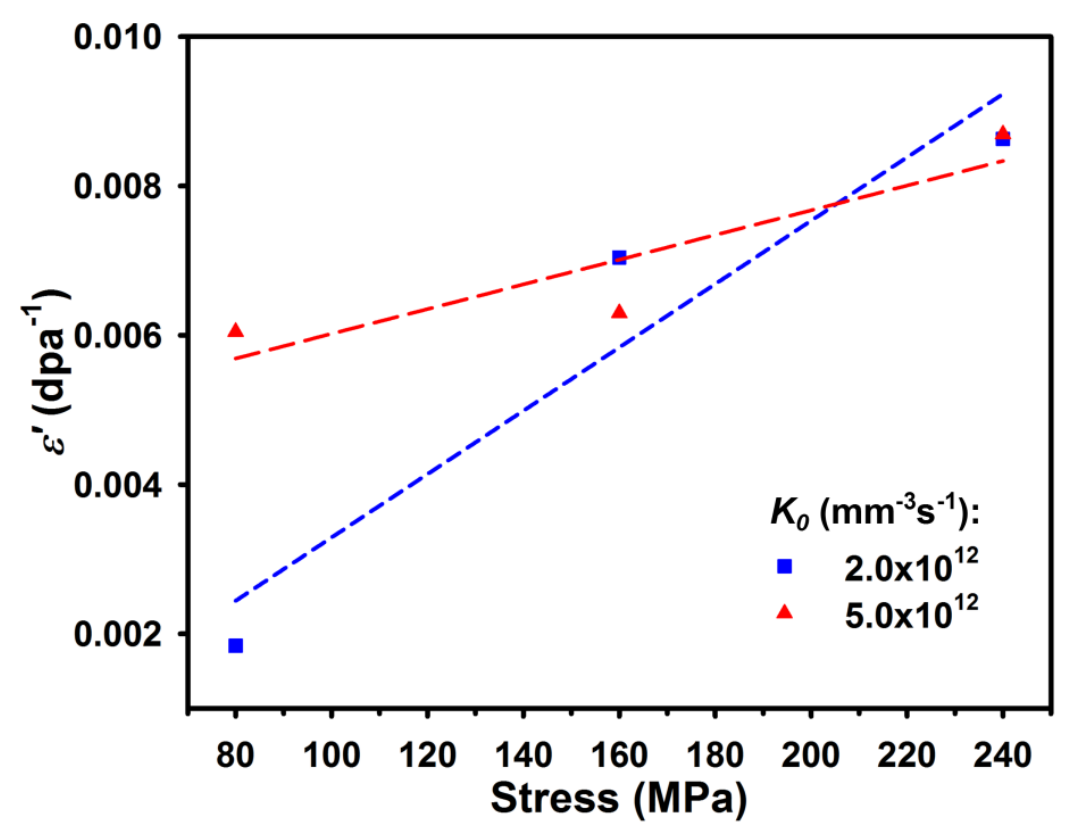

Figure 39. Creep rates as a function of stress. 
Figure 39 shows the variation of $\varepsilon^{\prime}$ with applied stress for different $K_{0} . \varepsilon^{\prime}$ increases with increase in applied stress. A linear regression fit of $\varepsilon^{\prime}$ (w.r.t. stress) was used in order to measure the creep compliance. From simulations, $B_{0}=4.24 \times 10^{-5} \mathrm{dpa}^{-1} \mathrm{MPa}^{-1}$ for $K_{0}=2.0 \times 10^{12} \mathrm{~mm}^{-3} \mathrm{~s}^{-1}$, and $B_{0}=1.65 \times 10^{-5} \mathrm{dpa}^{-1} \mathrm{MPa}^{-1}$ for $K_{0}=5.0 \times 10^{12} \mathrm{~mm}^{-3} \mathrm{~s}^{-1} . B_{0}$ is of the same order of magnitude as that seen in experiments for oxide dispersion strengthened ferritic steels at similar temperatures [221], but higher by a factor of 2-4. Xu and Was [212] performed irradiation creep experiments on a similar $9 \mathrm{Cr}-1 \mathrm{Mo}$ steel at $773 \mathrm{~K}$. While they did not measure the creep compliance, they did measure the creep rate, $\varepsilon^{\prime}$, at $160 \mathrm{MPa}$. Again, the creep rates predicted from our simulations are of the same order of magnitude, but higher by a factor of $\sim 3$ 4. The simulated radiation damage rates are similar to their dpa rates. In our model, the absorption efficiency of interstitials at dislocations, $z_{i}^{\alpha}$, and the GB sink strength, $k_{i G B}$, may be calibrated to fit the creep strain rates and dpa rates to experiments.

\subsection{Summary}

The constitutive framework developed in Chapter 2 was used to model the mechanical behavior and deformed microstructure during stress-controlled creep loading with concurrent irradiation. A GB sink model was used to account for the absorption of point defects at GBs. The framework was used to correlate the creep strain with the in-situ radiation dpa level for different defect pair generation rates. The substructure was studied in terms of the evolution of interstitial loop densities and sizes. While the interstitial loop densities increase monotonically with ongoing radiation, the loop sizes were found to saturate following the initial transients. The creep strain rate was found to increase with decreasing density of GBs and lower GB sink strengths. Irradiation creep rates and creep compliances were measured from simulations and compared to available experimental data for similar ferritic/martensitic steels. They were found to be of the same order of magnitude, but slightly higher than the experimental values.

This framework may be used to study the effect of various microstructural parameters (grain size, density of GBs, GB sink strengths, etc.) on the irradiation creep rates. Model predictions may be used to guide the engineering of structural materials with optimal creep properties. Future work needs to address the incorporation of spatial point defect gradients in a computationally feasible manner in order to capture the material physics more accurately. Models for failure initiation due to the absorption of point defects and radiation-induced segregation at GBs may also be developed as an extension of this work. 


\section{CHAPTER 6}

\section{CONSTITUTIVE EQUATIONS FOR MODELING NON-SCHMID}

\section{EFFECTS IN SINGLE CRYSTAL BCC-IRON}

\subsection{Introduction}

Until now, we have discussed deformation mechanisms in irradiated bcc metals at intermediate and elevated temperatures. While these mechanisms would also extend to the low temperature deformation behavior, non-Schmid effects are expected to be dominant in bcc metals at low temperatures. At these temperatures, inelastic deformation is characterized by complex slip modes, governed by the core spreading of $1 / 2<111\rangle$ screw dislocations and leading to non-Schmid yield behavior [57,222]. Atomistic simulations show that the core structure of the screw dislocation in bcc crystals is not confined to its slip plane but splits into several planes of the <111> zone, significant of which are the $\{110\}$ family of planes [223,224]. This phenomenon, commonly known as the core spreading effect, severely limits the mobility of screw dislocations in bcc metals and is the rate limiting deformation mechanism; the dislocation core must again constrict for it to glide. In violation of the Schmid's law, the yield stress depends not only on the resolved shear stress on the primary slip system, but also on stress components on the secondary 'non-glide' slip systems (necessary for the dislocation core to constrict) in these crystals $[54,122,225,226]$. Further, the plastic flow is non-associative, meaning that the yield potential and plastic flow potential are not identical [55,119,122,227]. Tension-compression asymmetry is one manifestation of this effect, particularly in terms of initial yield. Dislocation core spreading and non-Schmid effects are prevalent in majority of the bcc metals at low temperatures, observed for example, in Fe [1,228,229], Mo [226,227,230,231], Nb and Nb-Mo alloys [232-234], Ta and Ta-Re alloys [235].

This Chapter develops constitutive equations to model non-Schmid effects due to the said core spreading phenomena in bcc-Fe at low and ambient temperatures. The constitutive equations are incorporated in the previously developed dislocation density based crystal plasticity framework for bcc metals to model the mechanical behavior of Fe single crystals over a range of loading orientations and temperatures. While the effect of irradiation damage on the non-Schmid yield behavior is not entirely clear (not much research has been done in this area), we will comment on possible effects. 


\subsection{Background}

In order to account for the influence of non-Schmid stress components on initial yield, Qin and Bassani [54] proposed an analytical expression for the critical yield stress, $\tau_{c r}$, given by a linear combination of the resolved shear stress and the non-Schmid stresses on slip system $\alpha$, as a first order approximation, i.e.,

$$
\tau_{c r}=\tau^{\alpha}+\sum_{i=1}^{N_{n s}} a_{i} \tau_{i}^{\alpha}
$$

where $\tau^{\alpha}$ is the resolved shear stress on slip system $\alpha, a_{i}$ is the non-Schmid coefficient corresponding to the $i^{t h}$ non-Schmid stress component, $\tau_{i}^{\alpha}$, and $N_{n s}$ is total number of nonSchmid stress components. Based on atomistic simulations of an isolated $1 / 2<111\rangle$ screw dislocation on the $\{110\}$ slip plane in Mo and W [226], this yield criterion adopted for bcc crystals has the following form:

$$
\tau_{c r}=\boldsymbol{m}^{\alpha} \cdot \boldsymbol{\sigma} \cdot \boldsymbol{n}^{\alpha}+a_{1} \boldsymbol{m}^{\alpha} \cdot \boldsymbol{\sigma} \cdot \boldsymbol{n}_{n s}^{\alpha}+a_{2}\left(\boldsymbol{n}^{\alpha} \times \boldsymbol{m}^{\alpha}\right) \cdot \boldsymbol{\sigma} \cdot \boldsymbol{n}^{\alpha}+a_{3}\left(\boldsymbol{n}_{n s}^{\alpha} \times \boldsymbol{m}^{\alpha}\right) \cdot \boldsymbol{\sigma} \cdot \boldsymbol{n}_{n s}^{\alpha}
$$

where $\boldsymbol{\sigma}$ is the current stress state, $\boldsymbol{m}^{\alpha}$ and $\boldsymbol{n}^{\alpha}$ are unit vectors corresponding to the slip direction and the slip plane normal on slip system $\alpha, \boldsymbol{n}_{n s}^{\alpha}$ is the unit vector of the 'non-glide' plane on which the screw dislocation core was initially spread, and, $a_{1}, a_{2}$ and $a_{3}$ are coefficients that can be fit to the atomistically/experimentally determined yield stress for the bcc single crystal loaded in different orientations. The terms on the RHS of the Eq. (6.2) represent the resolved shear stress along the Schmid plane, resolved shear stress along the 'non-glide' plane, resolved stress normal to the Schmid plane, and the resolved stress normal to the "nonglide' plane, respectively. For the case of conventional yield, only the resolved shear stress along the Schmid slip plane is dominant; $a_{1}=a_{2}=a_{3}=0$ for a Schmid-type conventional yield behavior.

In recent years, a number of continuum crystal plasticity frameworks have been developed to model non-Schmid effects in bcc crystals at low homologous temperatures [229,236-239]. These frameworks generally rely on the aforementioned non-Schmid coefficients (or its variants) to capture asymmetric yield behavior observed in bcc crystals. With the exception of Lim et al. [239], the non-Schmid coefficients used in these crystal plasticity models are generally measured from molecular statics simulations performed at $0 \mathrm{~K}$. However, experimental studies of single crystal bcc metals suggest that the contribution of non-Schmid stresses to the yield stress diminishes with increasing temperatures. For example, studies on single crystal $\mathrm{Fe}$ [1] show that as the temperature is increased from $143 \mathrm{~K}$, deviation of yield from Schmid's law decreases monotonically and becomes limited at $295 \mathrm{~K}$. Based on comparison to experiments, Lim et al. [239] showed that while the non-Schmid stresses are 
dominant in the yield behavior of single crystal Ta at $77 \mathrm{~K}$, they are not so significant at $273 \mathrm{~K}$ and $300 \mathrm{~K}$. Temperature dependence of the non-Schmid coefficients thus needs to be considered in the expression for yield in constitutive models.

It should also be noted that the non-Schmid coefficients derived from atomistics are based on simulations of an isolated screw dislocation in the lattice, meaning that the yield stress is simply the lattice frictional resistance that needs to be overcome to initiate the glide of an isolated screw dislocation; dislocation-dislocation interactions are not considered. Also, the yield criterion does not provide any information about the evolution of non-Schmid stresses past the point of initial yield. In the presence of the long range field of other dislocations, the contribution of non-Schmid stresses may be expected to diminish beyond the point of initial yield, more so because dislocation glide (plastic flow) is governed primarily by the Schmid stresses. These effects are not generally considered in the continuum crystal plasticity models mentioned above.

The present work develops constitutive equations for yield in bcc-Fe, accounting for the temperature dependence of non-Schmid coefficients (based on results from available experiments), and the evolution of non-Schmid stresses with inelastic deformation. These equations are coupled with existing models for thermally-activated dislocation glide kinetics and used in the crystal plasticity framework developed in Chapter 2 to model the orientation and temperature dependence of yield and inelastic flow in single crystal Fe. Some of the constitutive equations presented in Chapter 2 are restated here as they have different implied meanings.

\subsection{Temperature dependence of non-Schmid coefficients}

As mentioned earlier, inelastic deformation in bcc crystals occurs due to the glide of $1 / 2<111>$ screw dislocations via kink pair formation on the $\{110\}$ family of planes. The 'nonSchmid' plane corresponding to each of the possible slip systems in bcc crystals is given in Gröger et al. [226]. It should be noted that the present work deals with modeling the deformation of bcc crystals at low homologous temperatures, in particular room temperature and below. There are contradictory claims in the literature regarding the nature of active slip systems in bccFe at these temperatures. Though majority of the studies [229,240-242] observed that elementary slip occurs via kink-pair formation on the $\{110\}$ plane at room temperature and below, Brunner and Diehl [243,244] observed that while this is true at temperatures below $250 \mathrm{~K}$, kink-pair formation occurs on the $\{112\}$ plane at temperatures above $250 \mathrm{~K}$. Nonetheless, it has been found [240,242] that slip on the $\{112\}$ plane is a combination of elementary slip steps on $\{110\}$ planes. In the present work, we assume that $\{110\}$ planes are the primary slip planes in bcc-Fe. Further, in order to account for the twinning/anti-twinning asymmetry observed in bcc crystals (cf. [234]), forward and backward slip has been explicitly accounted for in Ref. [226] by considering the positive and negative slip directions as separate slip systems. Thus, in place of the $12\{110\}<111>$ slip systems, we now have 24 slip systems considering both the positive and 
negative slip directions. The non-Schmid planes corresponding to these slip systems are also given in Ref. [226].

The Schmid and non-Schmid stresses (on each slip system) scale linearly as a function of the uniaxial stress, proportional to the respective Schmid and 'non-Schmid' factors. At yield, the slip system with the highest Schmid factor is activated; 'non-Schmid' factors corresponding to the loading orientation can be easily obtained for this activated slip system. Orientationdependent variation of the uniaxial yield stress may thus be used to estimate the parameters, $a_{1}$, $a_{2}, a_{3}$, and $\tau_{c r}$, simultaneously either by solving a system of linear equations corresponding to each orientation for Eq. (6.2), or using a regression analysis. However, this method predicted aphysical values of the parameters when fitted to experimental data. For example, $\tau_{c r}$ was predicted to be negative or extremely small $\left(\tau_{c r} \leq 0.05 \sigma_{Y}\right)$ in many cases, which is not possible; typically $\tau_{c r}$ is of the same order as $\sigma_{Y}$. Here $\sigma_{Y}$ is the yield stress. Further, $a_{1}$ was predicted to be negative in certain cases. As explained in Lim et al. [239], $a_{1}$, accounting for the twinning/anti-twinning asymmetry [226,231,234], should be non-negative in order to account for higher resolved shear stress along the anti-twinning direction. To avoid these anomalies, we determine $a_{1}$ separately by the procedure described below, and then solve for the rest of the parameters, $a_{2}, a_{3}$, and $\tau_{c r}$, simultaneously.

Given the threefold symmetry of a $1 / 2<111>$ screw dislocation in the bcc crystal (cf. [231] and references therein), orientation dependence of the yield stress about $\pm 30^{\circ}$ of one reference slip plane of the $\{110\}$ family is sufficient to determine the non-Schmid coefficients and may be applied to all slip planes. Note that $\pm 30^{\circ}$ correspond to the two $\{112\}$ planes adjacent to a $\{110\}$ plane along which twinning/anti-twinning asymmetry is observed.

$a_{1}$ may be estimated by fitting the yield stress, $\sigma_{Y}$, on the maximum resolved shear stress plane (MRSSP) measured as a function of the angle, $\chi$, which the MRSSP makes with the reference (110) slip plane about which the orientation dependence is measured (cf. $[225,226,228])$. This may be mathematically expressed as $[225,228]$

$$
\sigma_{Y}=\frac{\sigma^{0}}{\cos \chi+a_{1} \cos (\chi+\pi / 3)}
$$

where $\sigma^{0}$ is a material constant. Note that this expression is a simplified form of Eq. (6.2) obtained by clubbing $\tau_{c r}$, and the $a_{2}$ and $a_{3}$ terms, into $\sigma^{0}$. Transformation of the yield rule from the slip system tensorial description in Eq. (6.2) to the angular (i.e., $\chi$ ) dependence in Eq. (6.3) is a consequence of the bcc crystal symmetry and definition of the slip systems and corresponding non-Schmid slip planes [226]. 
Spitzig and Keh [1] performed experimental studies of the temperature dependence of the proportional limit stress in uniaxial tension, $\sigma_{p l}$, as a function of $\chi$ about the $(\overline{101})$ slip plane in single crystal Fe. These values extracted from Fig. 5 in [1] are given in Table 5. Assuming $\sigma_{Y} \approx \sigma_{p l}, a_{1}^{T}$ is estimated for different temperatures by fitting the experimental data in Table 5 to Eq. (6.3) using a least squares regression method, and is given in Table 5. The superscript $T$ on $a_{1}^{T}$ denotes the respective temperature. Based on the fit, $a_{1}^{T}$ is found to decrease monotonically as the temperature is increased from $143 \mathrm{~K}$ to $195 \mathrm{~K}$. Molecular statics simulations $[228,229]$ have estimated that $a_{1}^{0 \mathrm{~K}}=0.61$. From calculations, $a_{1}^{250 \mathrm{~K}} \approx 0.07 a_{1}^{0 \mathrm{~K}}$ and $a_{1}^{295 \mathrm{~K}} \approx 0.06 a_{1}^{0 \mathrm{~K}}$. This verifies that twinning/anti-twinning asymmetry, though present, is significantly diminished at ambient and room temperatures for Fe. Compiling the values of $a_{1}^{T}$ estimated over different temperatures, the variation of $a_{1}^{T}$ as a function of $T$ is shown in Fig. 40. Least square fit of these data points to an exponential decay function is also shown in Fig. 40, and has the following form:

$$
a_{1}^{T}=0.0106+2.3311 \exp (-0.0162 T) ; 143 \mathrm{~K} \leq T \leq 298 \mathrm{~K}
$$

Note that the value of $\sigma_{p l} / \sigma_{p l}\left(\chi=0^{\circ}\right)$ at $\chi= \pm 30^{\circ}$ was also reported for all temperatures in Ref. [1]. However, these data points did not give good fit to Eq. (6.3) for $T=143 \mathrm{~K}$ and $T=195 \mathrm{~K}$. Hence, these data points were excluded from the estimation of $a_{1}^{T}$ for all temperatures. As mentioned earlier, Eqs. (6.2) and (6.3) give the orientation dependence of yield on non-Schmid stresses to a first order approximation; higher order stress terms may be needed for fitting the non-Schmid coefficients over the entire range (even at $\chi= \pm 30^{\circ}$ ). Similar problems were also encountered when estimating $a_{1}$ from molecular statics results [228].

Having determined $a_{1}^{T}$, we are now left with three parameters: $\tau_{c r}^{T}, a_{2}^{T}, a_{3}^{T}$. Using the values of orientation-dependent yield stress, these parameters are estimated by solving a system of linear equations corresponding to different orientations for Eq. (6.2). Two sets of temperaturedependent non-Schmid parameters were obtained based on two different sets of experimental data for bcc-Fe [1,245]. These values are reported in Table 6 and plotted as function of temperature in Fig. 40. Note that the same value of $a_{1}^{T}$ (from Eq. (6.4)) was used in both these calculations. 
Table 5. Least squares fit of non-Schmid coefficient, $a_{1}$, to the experimentally measured variation of $\sigma_{p l}$ as a function of $\chi$ (from [1]) for different temperatures using Eq. (6.3).

\begin{tabular}{|c|c|c|c|c|}
\hline$T(\mathrm{~K})$ & $\chi\left(^{\circ}\right)$ & $\sigma_{p l} / \sigma_{p l}\left(\chi=0^{\circ}\right)$ & $a_{1}^{T}$ & $\sigma^{0} / \sigma_{p l}\left(\chi=0^{\circ}\right)$ \\
\hline \multirow[t]{4}{*}{143} & -24 & 1.02 & 0.2407 & 1.125 \\
\hline & 0 & 1 & & \\
\hline & 10 & 1.05 & & \\
\hline & 20 & 1.15 & & \\
\hline \multirow[t]{4}{*}{195} & -24 & 1.04 & 0.1154 & 1.05 \\
\hline & 0 & 1 & & \\
\hline & 10 & 1.03 & & \\
\hline & 20 & 1.08 & & \\
\hline \multirow[t]{4}{*}{250} & -24 & 1.08 & 0.0414 & 1.02 \\
\hline & 0 & 1 & & \\
\hline & 10 & 1.03 & & \\
\hline & 20 & 1.05 & & \\
\hline \multirow[t]{4}{*}{295} & -24 & 1.08 & 0.0363 & 1.015 \\
\hline & 0 & 1 & & \\
\hline & 10 & 1.02 & & \\
\hline & 20 & 1.04 & & \\
\hline
\end{tabular}




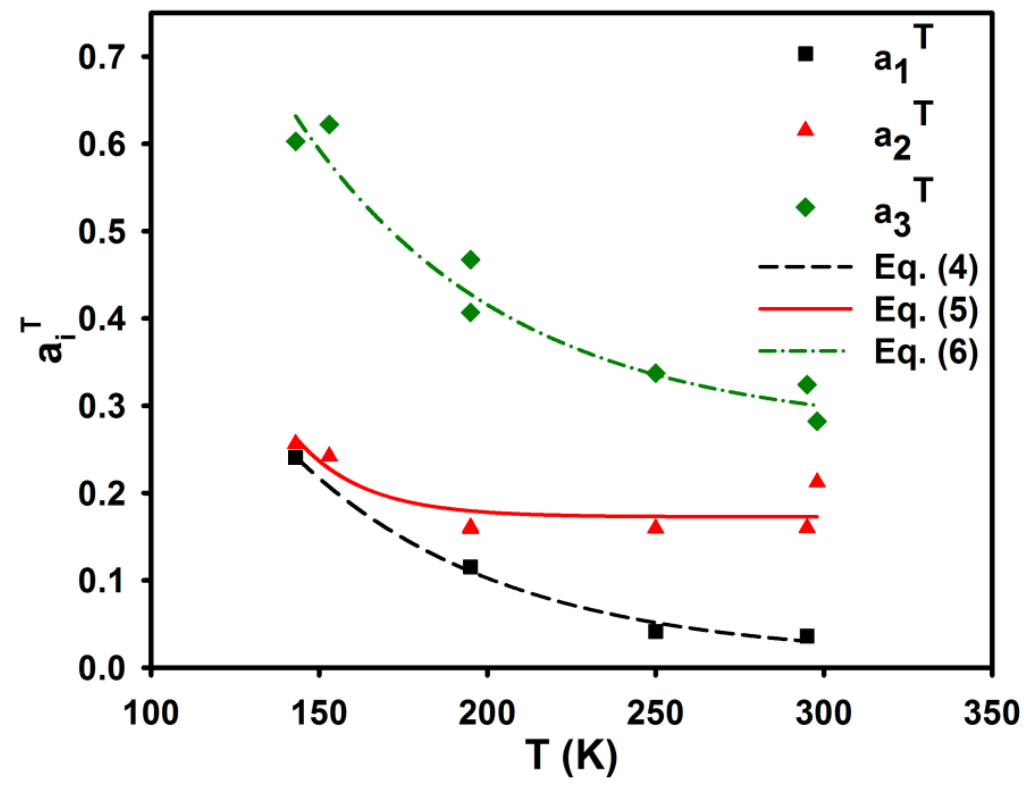

Figure 40. Variation of non-Schmid coefficients with temperature.

Except for the value of $a_{2}^{T}$ in the temperature range 295-298 K, the calculated values of $a_{2}^{T}$ and $a_{3}^{T}$ are comparable from both experimental data sets at similar temperatures, though the non-Schmid coefficients calculated from Takeuchi [245] are generally higher than that calculated from Spitzig and Keh [1]. This might be due to the fact that the lower yield stress was reported in [245], while the proportional limit stress was reported in [1]. In terms of deformation, the point of proportional limit occurs prior to the lower yield point. Physically, the non-Schmid stresses assist in constricting the (already spread) screw dislocation cores for glide to initiate (i.e., yield to occur) in bcc crystals. Full constriction of the dislocation cores might not have occurred at proportional limit, thus reflecting in slightly lower non-Schmid contributions as compared to that at lower yield point.

It can be seen from Table 6 that both $a_{2}^{T}$ and $a_{3}^{T}$ decrease with increase in temperature, and start saturating in the range $250-298 \mathrm{~K}$ ( similar to $a_{1}^{T}$ ). However, the contribution of these non-Schmid components to yield is still significant at ambient and room temperatures, in contrast to the near-negligible contribution of the twinning-antitwinning asymmetry component. Temperature dependence of $a_{2}^{T}$ and $a_{3}^{T}$, fit to exponential decay functions, are given in Eqs. (6.5) and (6.6), respectively.

$$
\begin{gathered}
a_{2}^{T}=0.1727+108.9126 \exp (-0.0496 T) ; 143 \mathrm{~K} \leq T \leq 298 \mathrm{~K} \\
a_{3}^{T}=0.2699+3.5454 \exp (-0.0160 T) ; 143 \mathrm{~K} \leq T \leq 298 \mathrm{~K}
\end{gathered}
$$


From atomistic simulations [229], $a_{2}^{0 \mathrm{~K}}=0.23$ and $a_{3}^{0 \mathrm{~K}}=0.55$. These values are marginally lower than the calculated corresponding non-Schmid parameters at $143 \mathrm{~K}$. Physically, these non-Schmid components represent the contribution of shear stresses perpendicular to the slip direction in constricting the distorted dislocation core [226]. Calculated values of these coefficients from (isolated dislocation) atomistic simulations might have been lower because dislocation-dislocation interactions in distorting the core are not accounted for in the atomistic simulations. Unlike $a_{1}^{T}, a_{2}^{T}$ and $a_{3}^{T}$ are not negligible at $250 \mathrm{~K}$ and $295 \mathrm{~K}$. Physically, this means that resolved stresses normal to the Schmid and the 'non-glide' plane still contribute to the constriction of screw dislocation cores (for glide to initiate) at ambient temperatures.

Table 6. Estimate of $a_{2}^{T}, a_{3}^{T}$, and $\tau_{c r}^{T}$ by fitting the orientation-dependent yield stress to Eq. (6.2) for different temperatures.

\begin{tabular}{lllllll}
\hline $\begin{array}{l}\text { Experimental } \\
\text { data }\end{array}$ & $\dot{\varepsilon}\left(\mathrm{s}^{-1}\right)$ & $T(\mathrm{~K})$ & $a_{2}^{T}$ & $a_{3}^{T}$ & $\tau_{c r}^{T}(\mathrm{MPa})$ & $\begin{array}{l}\text { Stress } \\
\text { measure }\end{array}$ \\
\hline $\begin{array}{l}\text { Spitzig and } \\
\text { Keh [1] }\end{array}$ & $5.6 \times 10^{-4}$ & 143 & 0.2564 & 0.6028 & 95.60 & $\begin{array}{l}\text { Proportional } \\
\text { limit }\end{array}$ \\
& & 195 & 0.1590 & 0.4069 & 54.57 & \\
& & 250 & 0.1595 & 0.3373 & 23.09 & \\
& & 295 & 0.1601 & 0.3243 & 12.97 & \\
$\begin{array}{l}\text { Takeuchi } \\
\text { [245] }\end{array}$ & $4.0 \times 10^{-3}$ & 153 & 0.2422 & 0.6222 & 116.87 & Lower yield \\
& & 195 & 0.1609 & 0.4673 & 83.55 & \\
& 298 & 0.2124 & 0.2822 & 22.97 & \\
\hline
\end{tabular}

The values of $\tau_{c r}^{T}$ calculated from [245] are, in general, higher as compared to that calculated from [1] at similar temperatures. This again is due to the fact that the lower yield stress was reported in [245], while the proportional limit stress was reported in [1]; stress levels would be comparatively lower at proportional limit. Further, the loading rate in [245] is an order of magnitude higher than that in [1], possibly leading to a higher value of $\tau_{c r}^{T}$. Physically, $\tau_{c r}^{T}$ represents the total lattice resistance due to the intrinsic frictional resistance and the obstacle resistance. Implications of this in modeling the post-yield inelastic flow are discussed later.

Using atomistic simulations of a $1 / 2<111>$ screw dislocation in bcc-Fe [229], the yield stress criterion given in Eq. (6.2) may be extended to account for the effect of 
tension/compression perpendicular to the glide direction and also account for the fact that hydrostatic stress does not contribute to dislocation glide. This leads to three additional terms in the expression for the critical yield stress [229]. For simplicity, the effect of these additional nonSchmid stresses on yield behavior is not considered the present work.

\subsection{Crystal plasticity framework}

The finite deformation crystal plasticity model is based on the multiplicative deformation of the deformation gradient, $\boldsymbol{F}$, into elastic, $\boldsymbol{F}^{e}$, and inelastic, $\boldsymbol{F}^{i}$, parts $[46,47]$. The inelastic velocity gradient, $\boldsymbol{L}^{i}$, given by $\boldsymbol{L}^{i}=\dot{\boldsymbol{F}}^{i} \cdot \boldsymbol{F}^{i-1}$, is the tensor sum of the crystallographic shearing rate, $\dot{\gamma}^{\alpha}$, over all slip systems, $N_{s}$, i.e.,

$$
\boldsymbol{L}^{i}=\sum_{\alpha=1}^{N_{s}} \dot{\gamma}^{\alpha} \boldsymbol{m}_{0}^{\alpha} \otimes \boldsymbol{n}_{0}^{\alpha}
$$

where $\boldsymbol{m}_{0}^{\alpha}$ and $\boldsymbol{n}_{0}^{\alpha}$ are unit vectors corresponding to the slip and slip plane normal directions in the reference (or intermediate) configuration. Shearing rate, $\dot{\gamma}^{\alpha}$, is a function of the resolved shear stress, $\tau^{\alpha}$, and the internal state variables (ISVs) on each slip system $\alpha$. The slip system dislocation density, $\rho^{\alpha}$, given as the additive sum of mobile, $\rho_{M}^{\alpha}$, and immobile dislocations, $\rho_{I}^{\alpha}$, i.e., $\rho^{\alpha}=\rho_{M}^{\alpha}+\rho_{I}^{\alpha}$, is used as an ISV in this framework.

\subsubsection{Dislocation glide kinetics}

Thermally activated glide of $1 / 2<111>$ screw dislocations by kink-pair nucleation is the rate controlling mechanism governing inelastic deformation in bcc metals at low temperatures (cf. [246]). In continuum constitutive formulations, the crystallographic shearing rate due to thermally activated dislocation glide is generally represented using a Kocks-type activation enthalpy driven flow rule [49], i.e.,

$$
\dot{\gamma}^{\alpha}=\left\{\begin{array}{l}
\dot{\gamma}_{0} \exp \left(-\frac{\Delta F_{g}}{k T}\left(1-\left(\frac{\tau_{f}^{\alpha}-s^{\alpha}}{s_{0}^{\alpha}}\right)^{p}\right)^{q}\right) ; \text { for } \tau_{f}^{\alpha}>s^{\alpha} \\
0 ; \text { for } \tau_{f}^{\alpha} \leq s^{\alpha}
\end{array}\right.
$$

where $\dot{\gamma}_{0}$ is the pre-exponential factor, $\Delta F_{g}$ is the activation barrier for dislocation glide in the absence of external stress, $k$ is Boltzmann constant, $T$ is absolute temperature, $\tau_{f}^{\alpha}$ is the driving stress for dislocation glide, $s^{\alpha}$ is the lattice resistance to dislocation glide, $s_{0}^{\alpha}$ is the lattice resistance at $0 \mathrm{~K}$, and $p$ and $q$ are parameters used to model the shape of the activation enthalpy function. Note that the double-kink nucleation mechanism in bcc metals is governed by two 
different temperature regimes (cf. [246]). For simplicity, these two regimes are not considered explicitly in the present work.

$s^{\alpha}$ may be expressed as the additive sum of contributions from the lattice frictional resistance, $s_{\text {fric }}^{T}$, and the obstacle resistance, $s_{o b s}^{\alpha}$, i.e.,

$$
s^{\alpha}=s_{f r i c}^{T}+s_{o b s}^{\alpha}
$$

$s_{\text {fric }}^{T}$ is the short range lattice resistance and is generally a function of the temperaturedependent Peierls stress in bcc metals. The long range resistance, $s_{o b s}^{\alpha}$, due to other defects, may be expressed as a function of the dislocation density using a Taylor hardening model, i.e.,

$$
s_{o b s}^{\alpha}=G b \sqrt{\sum_{\zeta=1}^{N_{s}} q_{\rho} A^{\alpha \zeta} \rho^{\zeta}}
$$

where $G$ is the shear modulus, $b$ is the Burgers vector magnitude, $q^{\rho}$ is the dislocation barrier strength, and, $A^{\alpha \zeta}$ is the matrix of slip system dislocation interaction coefficients. Physically, $s^{\alpha}$ should be equal to $\tau_{c r}^{T}$ at yield, since $\tau_{c r}^{T}$ represents the total slip system resistance to glide initiation.

Strictly speaking, the driving stress for dislocation glide, $\tau_{f}^{\alpha}$, must comprise solely of the resolved shear stress, $\tau^{\alpha}$, on slip system $\alpha$; this must hold whether yield (i.e., glide initiation) follows Schmid's law or not. Further, crystal viscoplastic formulations do not generally model yield and inelastic flow separately. Yield is implicitly modeled in the flow rule using the lattice resistance, $s^{\alpha}$, or a similar threshold-like term.

In the present formulation, we assume the initial driving stress to be the additive sum of the resolved shear stress, $\tau^{\alpha}$, and the contribution from non-Schmid stresses, $\sigma_{n s}^{\alpha}$, i.e.,

$$
\left.\tau_{f}^{\alpha}\right|_{\text {initial }}=\tau^{\alpha}+\sigma_{n s}^{\alpha}
$$

From Eq. (6.2), $\sigma_{n s}^{\alpha}$ has the following form:

$$
\sigma_{n s}^{\alpha}=a_{1}^{T} \boldsymbol{m}^{\alpha} \cdot \boldsymbol{\sigma} \cdot \boldsymbol{n}_{n s}^{\alpha}+a_{2}^{T}\left(\boldsymbol{n}^{\alpha} \times \boldsymbol{m}^{\alpha}\right) \cdot \boldsymbol{\sigma} \cdot \boldsymbol{n}^{\alpha}+a_{3}^{T}\left(\boldsymbol{n}_{n s}^{\alpha} \times \boldsymbol{m}^{\alpha}\right) \cdot \boldsymbol{\sigma} \cdot \boldsymbol{n}_{n s}^{\alpha}
$$

Eq. (6.11) thus satisfies the yield law given in Eq. (6.2). However, Eq. (6.11) needs to be modified beyond the point of yield such that the contribution from non-Schmid stresses diminishes with inelastic deformation. From a physical standpoint, it is unlikely that the nonSchmid effects would suddenly disappear; not all dislocations start gliding at the point of initial yield, meaning that not all dislocation cores get constricted at yield. Further, long range interaction fields with other dislocations may be expected to limit the extent of dislocation core spreading and its subsequent constriction. We propose to model this by assuming decay of the 
non-Schmid contribution to the driving stress as an exponential function of the effective inelastic strain, $\varepsilon_{e f f}^{i}$, i.e.,

$$
\tau_{f}^{\alpha}=\tau^{\alpha}+\sigma_{n s}^{\alpha} \exp \left(-\frac{\varepsilon_{e f f}^{i}}{\varepsilon_{0}^{i}}\right)
$$

where $\varepsilon_{0}^{i}$ is a material constant. A concomitant effect of this constitutive rule is that as the contribution of non-Schmid stresses decays with increasing inelastic strain, the resolved shear stress, $\tau^{\alpha}$, dominates the driving force for dislocation glide.

Note that the non-Schmid stresses may be assumed to decay as a function of the dislocation density, $\rho^{\alpha}$, or some other state variable. However, in order to maintain general applicability to any framework, we choose the constitutive form given in Eq. (6.13); $\varepsilon_{\text {eff }}^{i}$ is the most generic state variable in all viscoplastic formulations. Detailed experimental studies, for example, of the orientation dependence of yield in pre-strained crystals, are needed to determine the exact nature of the decay of non-Schmid stresses with applied strain.

\subsubsection{Dislocation evolution}

We have developed constitutive equations for the evolution of mobile and immobile dislocations in bcc metals subjected to radiation damage in Chapter 2. In these constitutive equations, an additional ISV was used to model the material defects generated due to irradiation (primarily interstitial loops in bcc). Neglecting terms representing the interaction of dislocations with irradiation-induced defects, the rate of evolution of mobile and immobile dislocations, as a function of the crystallographic shearing rate, $\dot{\gamma}^{\alpha}$, is given as [16]

$$
\begin{gathered}
\dot{\rho}_{M}^{\alpha}=\frac{k_{m u l}}{b} \sqrt{\sum_{\varsigma} \rho^{\varsigma}}\left|\dot{\gamma}^{\alpha}\right|-k_{c s} \exp \left(-\frac{\left(\tau^{*}-\left|\tau^{\chi}\right|\right) V_{a}}{k T}\right) \rho_{M}^{\alpha}\left|\dot{\gamma}^{\alpha}\right|-\frac{2 R_{c}}{b} \rho_{M}^{\alpha}\left|\dot{\gamma}^{\alpha}\right|-\frac{1}{b \lambda^{\alpha}}\left|\dot{\gamma}^{\alpha}\right| \\
\dot{\rho}_{I}^{\alpha}=\frac{1}{b \lambda^{\alpha}}\left|\dot{\gamma}^{\alpha}\right|-k_{d y n} \rho_{I}^{\alpha}\left|\dot{\gamma}^{\alpha}\right|
\end{gathered}
$$

The 1 st term in Eq. (6.14) represents the rate of formation of mobile dislocations by multiplication at existing dislocation segments, the $2^{\text {nd }}$ term represents the rate of migration of mobile dislocations by cross-slip (from slip plane $\alpha$ to $\chi$ ), the $3^{\text {rd }}$ term represents the rate of mutual annihilation of mobile dislocation segments with opposite Burgers vectors within a critical capture radius $R_{c}$, and the $4^{\text {th }}$ term represents the rate of immobilization of mobile dislocation segments by trapping at barriers, given as a function of the effective mean free path, $\lambda^{\alpha}=1 / \beta_{\rho} \sqrt{\rho^{\alpha}}$, of the gliding dislocations. The corresponding rate of addition of immobile dislocations due to trapping of mobile dislocations is reflected in the $1^{\text {st }}$ term of Eq. (6.15), while 
the $2^{\text {nd }}$ term represents the rate of annihilation of immobile dislocations by dynamic recovery. In these equations, $k_{m u l}, k_{c s}$, and $k_{d y n}$ are material constants corresponding to mobile dislocation multiplication, mobile dislocation cross-slip, and dynamic recovery of immobile dislocations, respectively. $\tau^{*}$ is the activation stress for cross-slip over an activation volume, $V_{a}$, and $\beta_{\rho}$ is a constant associated with dislocation trapping. These equations are assumed to principally constitute the dislocation mechanisms governing inelastic deformation in bcc-Fe.

\subsection{Model parameters}

Temperature dependent elastic constants for bcc-Fe are adopted from [247]. As mentioned earlier, the material is assumed to have $24\{110\}<111>$ slip systems, considering both positive and negative slip. These slip systems are listed in [226]. Based on bcc crystallography, the Burgers vector magnitude of $\mathrm{Fe}$ is given as $b=(\sqrt{3} / 2) a_{0}$, where $a_{0}=0.2866 \mathrm{~nm}$ is the lattice parameter of bcc-iron [78].

Experimental studies [243,244,248,249] have suggested that the activation barrier for dislocation glide at zero stress, $\Delta F_{g}$, is in the range $0.65-0.9 \mathrm{eV}$. We have assumed that $\Delta F_{g}=0.7 \mathrm{eV}$ at all temperatures. Values of the shape function parameters, $p=0.28$ and $q=1.34$, are taken from [48], where they were used to model the tensile behavior of bcc-Ta. The pre-exponential factor in the flow rule, $\dot{\gamma}_{0}$, is assumed to be have a constant value of $5.0 \times 10^{4} \mathrm{~s}^{-1}$

Temperature dependence of the non-Schmid coefficients, $a_{1}^{T}, a_{2}^{T}$ and $a_{3}^{T}$, has already been derived in Section 6.3. The non-Schmid contribution to the driving stress has been modeled to decay as an exponential function of the effective inelastic strain in Eq. (6.13). The value of the associated material constant, $\varepsilon_{0}^{i}$, is assumed to be 0.02 , meaning that the non-Schmid contribution at $\varepsilon_{\text {eff }}^{i}=0.02$ decreases to $\approx 37 \%$ of the contribution at initial yield $\left(\varepsilon_{\text {eff }}^{i}=0\right)$. Systematic experiments/atomistic simulations are needed to further refine this phenomenological rule. Parametric study of the effect of $\varepsilon_{0}^{i}$ on the simulated stress-strain curves is shown in Section 6.3.

From [80], the lattice resistance at $0 \mathrm{~K}, s_{0}^{\alpha}=390 \mathrm{MPa}$. From experiments [250], temperature-dependent values of the lattice frictional resistance, $s_{\text {fric }}^{T}$, are: $s_{\text {fric }}^{295 \mathrm{~K}} \approx 10 \mathrm{MPa}$, $s_{\text {fric }}^{250 \mathrm{~K}} \approx 25 \mathrm{MPa}, s_{\text {fric }}^{195 \mathrm{~K}} \approx 60 \mathrm{MPa}$, and $s_{\text {fric }}^{143 \mathrm{~K}} \approx 115 \mathrm{MPa}$. These values were again fit to a polynomial expression to obtain the temperature dependence of $s_{f r i c}^{T}$, i.e., 


$$
s_{\text {fric }}^{T}=361.7426-2.2311 T[\mathrm{~K}]+0.0035 T[\mathrm{~K}]^{2} \mathrm{MPa}
$$

Further, $s_{\text {fric }}^{T}$ is assumed to be independent of the loading orientation. The dislocation barrier strength, $q^{\rho}$, is assumed to be 0.3 in the expression for obstacle resistance, $s_{o b s}^{\alpha}$. A similar value was used in Arsenlis et al. [12] to model the dislocation barrier resistance in irradiated $\mathrm{Cu}$. The values of the dislocation interaction coefficients were assumed to be $A^{\alpha \alpha}=1.0$ (self-interaction) and $A^{\alpha \varsigma}(\varsigma \neq \alpha)=0.2$ (latent interaction). From experiments $[248,250], s_{o b s}^{\alpha} \approx 5-8 \mathrm{MPa}$ at initial yield. Putting in values of the above mentioned parameters in Eq. (6.10), the initial value of dislocation density, $\rho^{0} \approx 4.0-10.0 \times 10^{4} \mathrm{~cm}^{-2}$. We have assumed that an equal population of mobile and immobile dislocations exists initially, and $\rho_{M}^{0}=\rho_{I}^{0}=4.0 \times 10^{4} \mathrm{~mm}^{-2}$ on all slip systems. 
Table 7. Model parameters for bcc-Fe.

\begin{tabular}{|c|c|c|}
\hline Parameter & Value & Meaning \\
\hline $\begin{array}{l}C_{11}, C_{12}, C_{44}, \\
v\end{array}$ & $\begin{array}{l}239260-24520 /(\exp (392.03 / T[\mathrm{~K}])-1) \mathrm{MPa} \\
135780-6550 /(\exp (469.65 / T[\mathrm{~K}])-1) \mathrm{MPa}, \\
120720-3500 /(\exp (162.9 / T[\mathrm{~K}])-1) \mathrm{MPa}, 0.29\end{array}$ & $\begin{array}{l}\text { Elastic constants } \\
\text { (from [247]) }\end{array}$ \\
\hline$G$ & $87600-17 T[\mathrm{~K}] \mathrm{MPa}$ & $\begin{array}{l}\text { Shear modulus } \\
\text { (from [251]) }\end{array}$ \\
\hline$a_{0}$ & $0.2866 \mathrm{~nm}$ & $\begin{array}{l}\text { Lattice parameter } \\
\text { (from [78]) }\end{array}$ \\
\hline$\Delta F_{g}, p, q, \dot{\gamma}_{0}$ & $0.7 \mathrm{eV}, 0.28,1.34,5.0 \times 10^{4} \mathrm{~s}^{-1}$ & $\begin{array}{l}\text { Flow rule } \\
\text { parameters }\end{array}$ \\
\hline$a_{1}^{T}, a_{2}^{T}, a_{3}^{T}$ & $\begin{array}{l}a_{1}^{T}=0.0106+2.3311 \exp (-0.0162 T) \\
a_{2}^{T}=0.1727+108.9126 \exp (-0.0496 T) \\
a_{3}^{T}=0.2699+3.5454 \exp (-0.0160 T) ; \text { for } \\
143 \mathrm{~K} \leq T \leq 298 \mathrm{~K}\end{array}$ & $\begin{array}{l}\text { Non-Schmid } \\
\text { parameters }\end{array}$ \\
\hline $\begin{array}{l}s_{\text {fric }}^{\alpha}, q^{\rho}, A^{\alpha \alpha}, \\
A^{\alpha \varsigma}(\varsigma \neq \alpha) \\
s_{0}^{\alpha}\end{array}$ & $\begin{array}{l}s_{\text {fric }}^{T}=361.7426-2.2311 T[\mathrm{~K}]+0.0035 T[\mathrm{~K}]^{2} \mathrm{MPa} \\
0.3,1.0,0.2,390 \mathrm{MPa}\end{array}$ & $\begin{array}{l}\text { Hardening } \\
\text { parameters }\end{array}$ \\
\hline$\varepsilon_{0}^{i}$ & 0.02 & $\begin{array}{l}\text { Parameter for } \\
\text { decay of non- } \\
\text { Schmid stresses }\end{array}$ \\
\hline $\begin{array}{l}k_{m u l}, k_{c s}, k_{d y n} \\
R_{c}, \beta_{\rho}\end{array}$ & $3.45 \times 10^{-2}, 0,2.75 \times 10^{2}, 5 b, 7.40 \times 10^{-2}$ & $\begin{array}{l}\text { Dislocation } \\
\text { evolution } \\
\text { parameters }\end{array}$ \\
\hline
\end{tabular}

Parameters associated with the dislocation evolution equations include $k_{m u l}, k_{c s}, k_{d y n}, \tau^{*}$, $V_{a}, R_{c}$, and $\beta_{\rho}$. Since this work focusses on modeling the mechanical behavior of bcc-Fe at low and ambient temperatures, dislocation migration due to thermally activated cross-slip may be neglected at these temperatures, i.e., $k_{c s}=0$ (see [16] for values of the parameters, $\tau^{*}$ and $V_{a}$, 
when $k_{c s}$ is non-zero). The critical capture radius, $R_{c}$, for mutual annihilation of dislocation dipoles is assumed to be $6 b(\approx 1.5 \mathrm{~nm})$. This is of the same order as that used in [12] for mutual dislocation dipole annihilation in irradiated $\mathrm{Cu}$. The remaining parameters, $k_{m u l}, k_{d y n}$ and $\beta_{\rho}$, were used to fit simulation results to the experimental single crystal stress-strain curves of bcc-Fe [4] for different loading orientations at $298 \mathrm{~K}$. Based on the fit (discussed in Section 6.6.1), $k_{m u l}=3.45 \times 10^{-2}, k_{d y n}=2.75 \times 10^{2}$, and $\beta_{\rho}=7.40 \times 10^{-2}$. Further, these parameters are assumed to be temperature invariant. Table 7 lists the values of the complete set of parameters.

\subsection{Results and discussion}

\subsubsection{Orientation-dependent stress-strain curves at $298 \mathrm{~K}$}

3D finite element simulations were performed using the finite element program ABAQUS [86]. Periodic boundary conditions were enforced in all directions on a cubic simulation cell of side $100 \mu \mathrm{m}$. The material was loaded in tension using displacementcontrolled boundary conditions at a strain rate of $10^{-4} \mathrm{~s}^{-1}$ (unless specified otherwise). The constitutive equations were implemented using the user material subroutine (UMAT) in ABAQUS.

The constitutive model was fit to the experimental initial hardening response (up to $\approx 5 \%$ strain) of single crystal iron loaded in three different orientations, [ $\left.\begin{array}{lll}0 & 0 & 1\end{array}\right],\left[\begin{array}{lll}0 & 1 & 1\end{array}\right]$ and [ $\left.\begin{array}{lll}1 & 1 & 1\end{array}\right]$, at 298 K. Figure 41 compares the simulated stress-strain curves with the experimental data [4,236]. A reasonable fit was obtained for the stress-strain response in all three orientations. However, the simulated yield stress for the [ $\left.\begin{array}{lll}\overline{1} & 1 & 1\end{array}\right]$ orientation was slightly off the experimental value. Note that the non-Schmid parameters were calculated from a different set of experiments [1,245]. Small differences in material composition might have resulted in different values of yield stress. For example, it has been shown [252] that ppm level carbon concentration difference can result in non-negligible difference in the yield stress of $\mathrm{Fe}$ single crystals, especially at low temperatures. If the experimental data from Ref. [4] were used to calculate the non-Schmid parameters, $a_{1}^{298 \mathrm{~K}}=0.0363, a_{2}^{298 \mathrm{~K}}=-0.0333, a_{3}^{298 \mathrm{~K}}=0.1417$, and $\tau_{c r}^{298 \mathrm{~K}}=17.87 \mathrm{MPa}$. Due to the lack of systematic data regarding the variation of orientation-dependent yield stress with temperature in [4], we have not used these experimental results for developing the constitutive equations for non-Schmid parameters. Nevertheless, the overall stress-strain response of the simulated material is in qualitative agreement with experiments. 


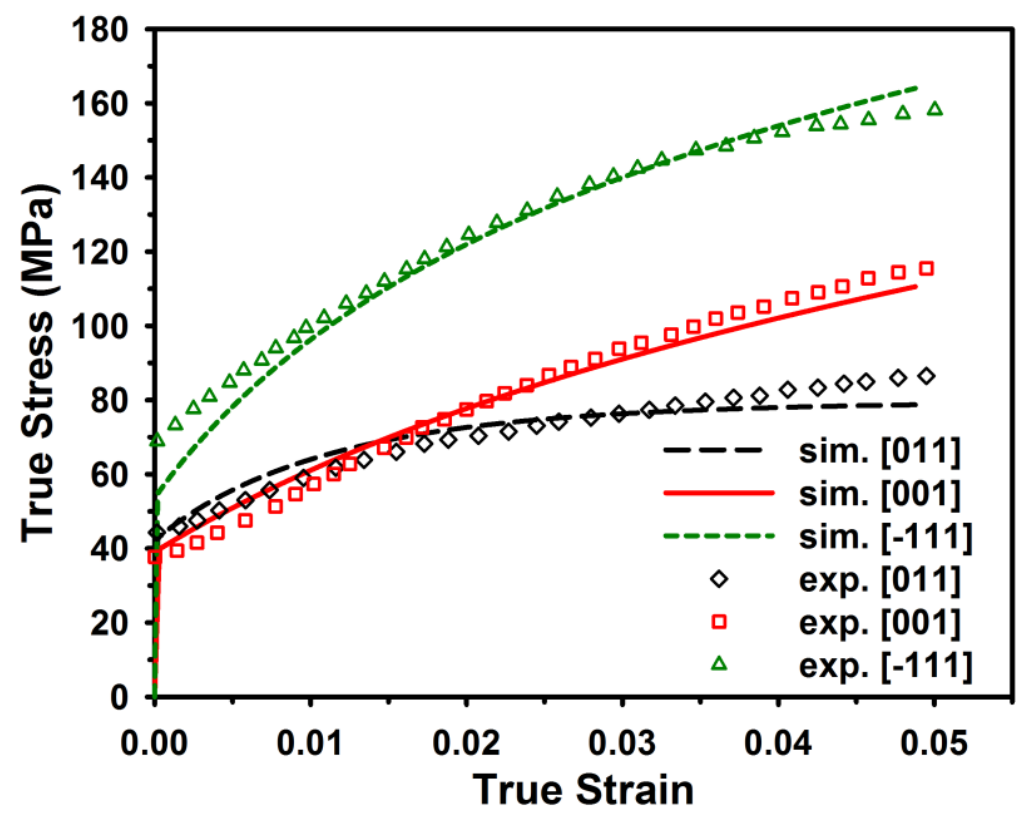

Figure 41. Comparison of simulations (solid and dotted lines) calibrated to the experimental stress-strain curves (open symbols) for three different orientations at $298 \mathrm{~K}$. Experimental data from [4].

Figure 42 shows the evolution of average mobile and immobile dislocation density as a function of strain for the three loading orientations. These dislocation densities are averaged over all slip systems. While the dislocation densities (both mobile and immobile) saturate for the $\left[\begin{array}{lll}0 & 1 & 1\end{array}\right]$ loading case, this is not the case for [ [ $\left.\begin{array}{lll}0 & 0 & 1\end{array}\right]$ and [ [ $\left.\begin{array}{lll}1 & 1 & 1\end{array}\right]$ loading. The hardening behavior in the stress-strain curves in Fig. 41 suggest the same (obstacle hardening is a function of dislocation density). Further, the mobile dislocation density for the [l $\left.\begin{array}{lll}0 & 1\end{array}\right]$ and [ $\left[\begin{array}{lll}1 & 1 & 1\end{array}\right]$ loading cases increases asymptotically. This dislocation evolution behavior is qualitatively similar to that seen in experiments [4]; the nature of dislocation evolution, asymptotic or saturating, is a function of the loading orientation and active slip systems. 


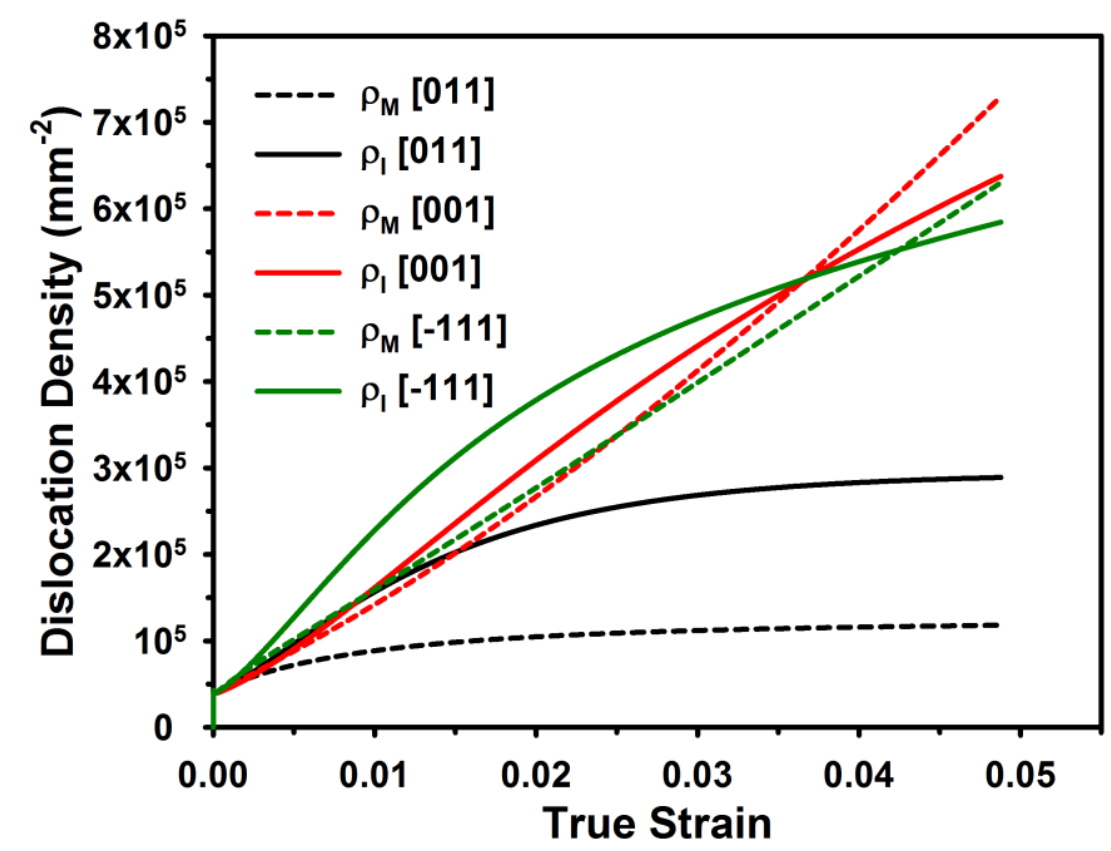

Figure 42. Evolution of average dislocation density as a function of strain for different loading orientations.

\subsubsection{Temperature dependent yield stress}

The constitutive equations were used to predict the yield stress of single crystal Fe for various temperatures and loading orientations. The model predictions were compared to the experimental data [1] for the material loaded in [1 116 , [ [ $\overline{1} 3$ 7], and [ $\overline{4}$ 9 9] orientations at 143 $\mathrm{K}, 195 \mathrm{~K}, 250 \mathrm{~K}$, and $295 \mathrm{~K}$. Figure 43 compares the $0.2 \%$ yield stress measured from simulations to the corresponding experimental data as a function of angle, $\chi$, which the MRSSP makes with the reference (110) slip plane. $\chi=-30^{\circ}, \chi=0^{\circ}$, and $\chi=30^{\circ}$ in Ref. [1] roughly correspond to the [lll 116$],\left[\begin{array}{lll}\overline{1} & 3 & 7\end{array}\right]$, and [ [ $\left.\begin{array}{ll}4 & 9\end{array}\right]$ loading orientations, respectively. Except for the [ $\overline{4} 9$ 9] loading, the orientation-dependent yield behavior was captured fairly accurately at all temperatures. The yield stress for [ $\overline{4} 99]$ orientation was over-predicted at all temperatures. As mentioned earlier in Section 6.3, the experimental yield stress for this orientation, $\chi=30^{\circ}$, was not used to estimate the non-Schmid coefficients; a higher order stress term may be necessary to capture the yield behavior for this orientation. Generally, this orientation is also excluded in estimation of non-Schmid parameters from atomistic simulations (cf. [228]). 


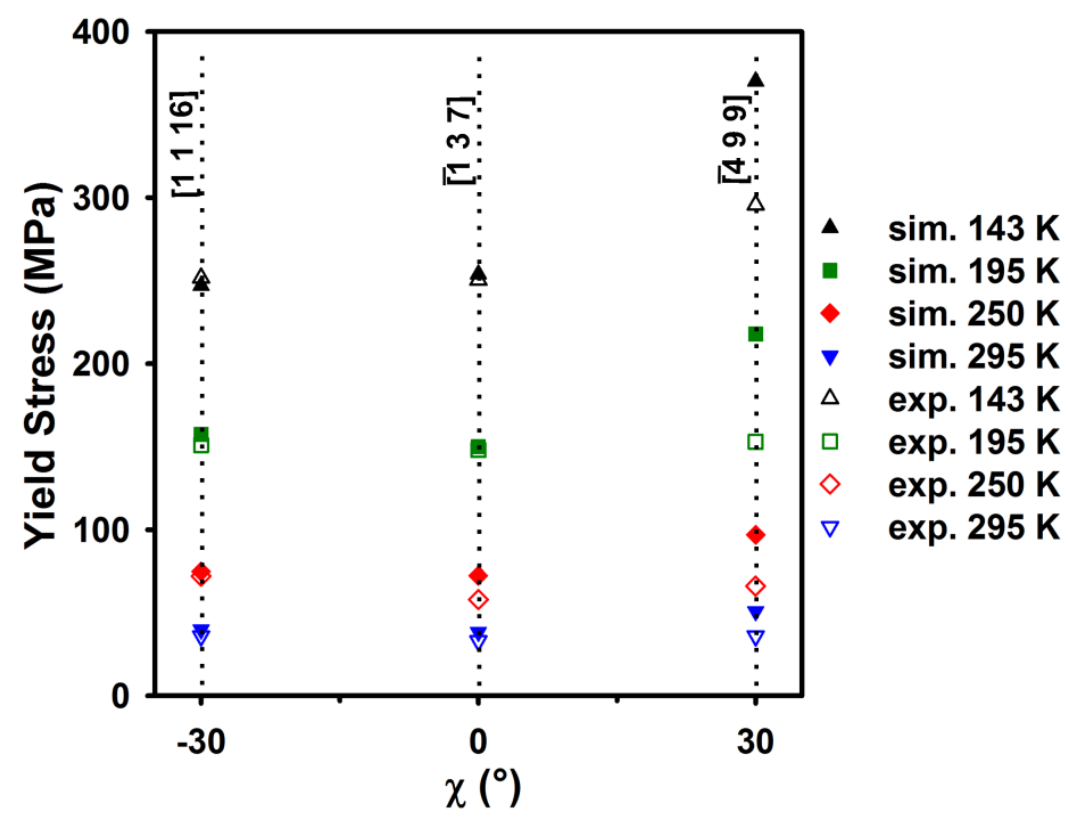

Figure 43. Comparison of yield stress measured from simulations (closed symbols) with experiments (open symbols) for different loading orientations and temperatures.

The yield stress, in the present case, is a function of the non-Schmid stresses, and the lattice frictional and obstacle resistances. Of these, the non-Schmid stresses are orientation and temperature dependent (cf. Eqs. (6.4)-(6.6)), while the lattice frictional resistance is assumed to be just temperature dependent (cf. Eq. (6.16)). Temperature dependence of the obstacle resistance was ignored in the present work; the simulated hardening response was, in some cases, not in agreement with the experimental hardening response at lower temperatures. Moreover, the hardening response was calibrated using a different set of experiments [4] at $298 \mathrm{~K}$. The simulated hardening may not be expected to fit the experimental results from Ref. [1] at lower temperatures.

A ratio of the total resolved stress to the Schmid stress (on slip system $\beta$ with the highest Schmid factor) given as

$$
R_{n s}^{T}=\frac{\boldsymbol{m}^{\beta} \cdot \boldsymbol{\sigma} \cdot \boldsymbol{n}^{\beta}+a_{1}^{T} \boldsymbol{m}^{\beta} \cdot \boldsymbol{\sigma} \cdot \boldsymbol{n}_{n s}^{\beta}+a_{2}^{T}\left(\boldsymbol{n}^{\beta} \times \boldsymbol{m}^{\beta}\right) \cdot \boldsymbol{\sigma} \cdot \boldsymbol{n}^{\beta}+a_{3}^{T}\left(\boldsymbol{n}_{n s}^{\beta} \times \boldsymbol{m}^{\beta}\right) \cdot \boldsymbol{\sigma} \cdot \boldsymbol{n}_{n s}^{\beta}}{\boldsymbol{m}^{\beta} \cdot \boldsymbol{\sigma} \cdot \boldsymbol{n}^{\beta}}
$$

may be used for estimating the deviation from 'conventional' Schmid behavior at temperature $T$. $R_{n s}^{T}$ is plotted for different temperatures in the standard stereographic triangle in Figs. 44 (a)-(d). Note that these values are calculated at initial yield. The corresponding Schmid factor is plotted in the Fig. 44 (e). The deviation from Schmid behavior increases with decrease in temperature. The value of $R_{n s}^{T}$, though relatively lower, is slightly greater than unity at $250 \mathrm{~K}$ and $295 \mathrm{~K}$. This 
suggests that non-Schmid effects are not negligible at room temperature. Furthermore, $R_{n s}^{T}$ is higher for crystallographic orientations between the $\left[\begin{array}{lll}0 & 0 & 1\end{array}\right]$ and $\left[\begin{array}{lll}\overline{1} & 1 & 1\end{array}\right]$ poles in the stereographic triangle. As one approaches the [ $\left[\begin{array}{lll}0 & 1 & 1\end{array}\right]$ pole, $R_{n s}^{T}$ gradually decreases to unity at all temperatures.
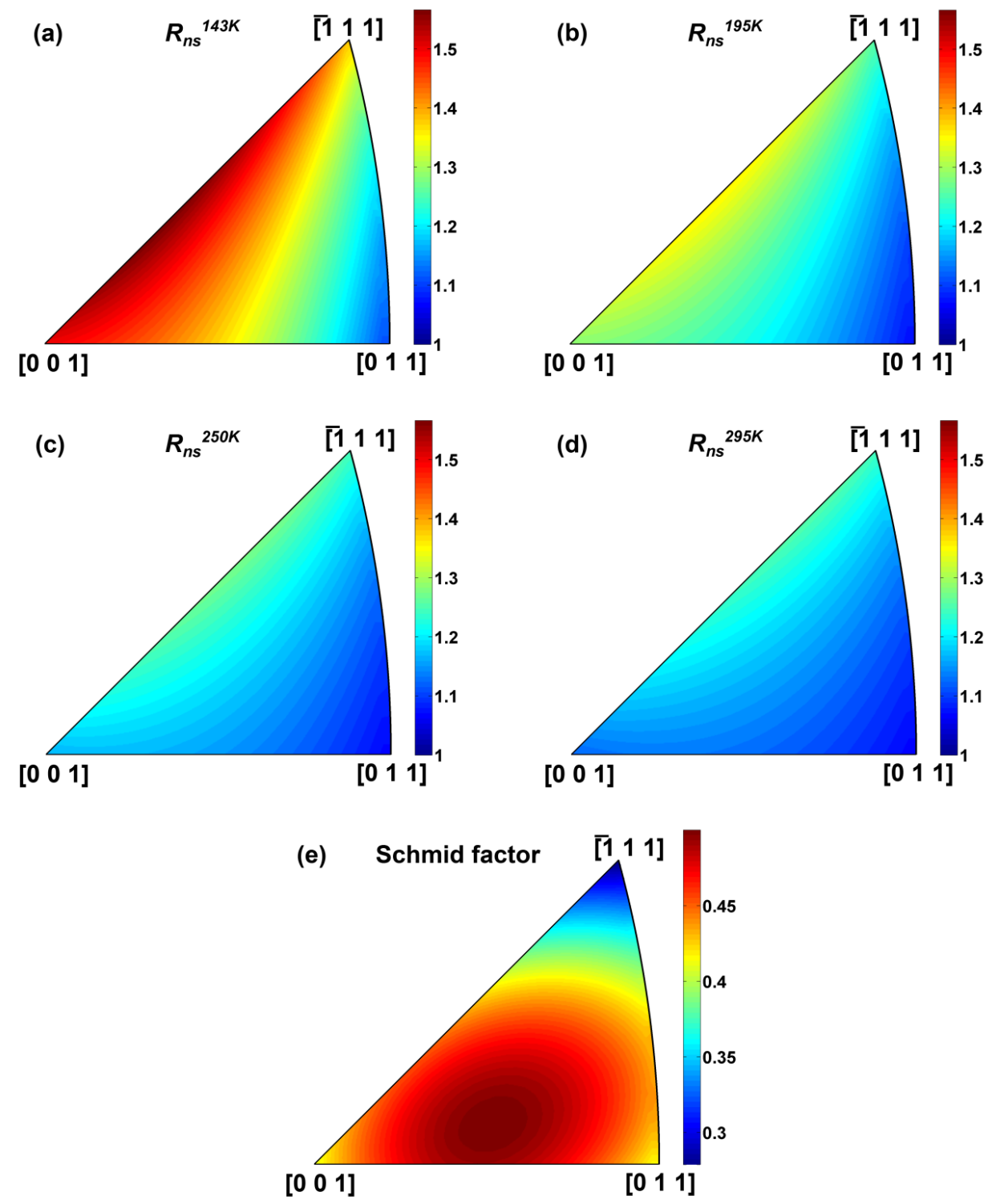

Figure 44. $R_{n s}^{T}$ for uniaxial tension plotted in the standard stereographic triangle at (a): $143 \mathrm{~K}$, (b): $195 \mathrm{~K},(\mathbf{c}): 250 \mathrm{~K}$, and (d): $295 \mathrm{~K}$. (e): Schmid factor for bcc metals. 


\subsubsection{Parametric study of $\varepsilon_{0}^{i}$}

The effect of $\varepsilon_{0}^{i}$ on the mechanical response of the specimen loaded in [1 116$]$ orientation was studied at $195 \mathrm{~K}$. The stress-strain response with no non-Schmid effects, i.e., associative flow was also simulated. Figure 45 shows the stress-strain curves for all these cases compared to the experimental data. The 'associative' legend represents no non-Schmid effects/associative flow, and the open symbols show the experimental stress-strain curve from Spitzig and Keh [1] for the [1 11 16] loading at $195 \mathrm{~K}$.

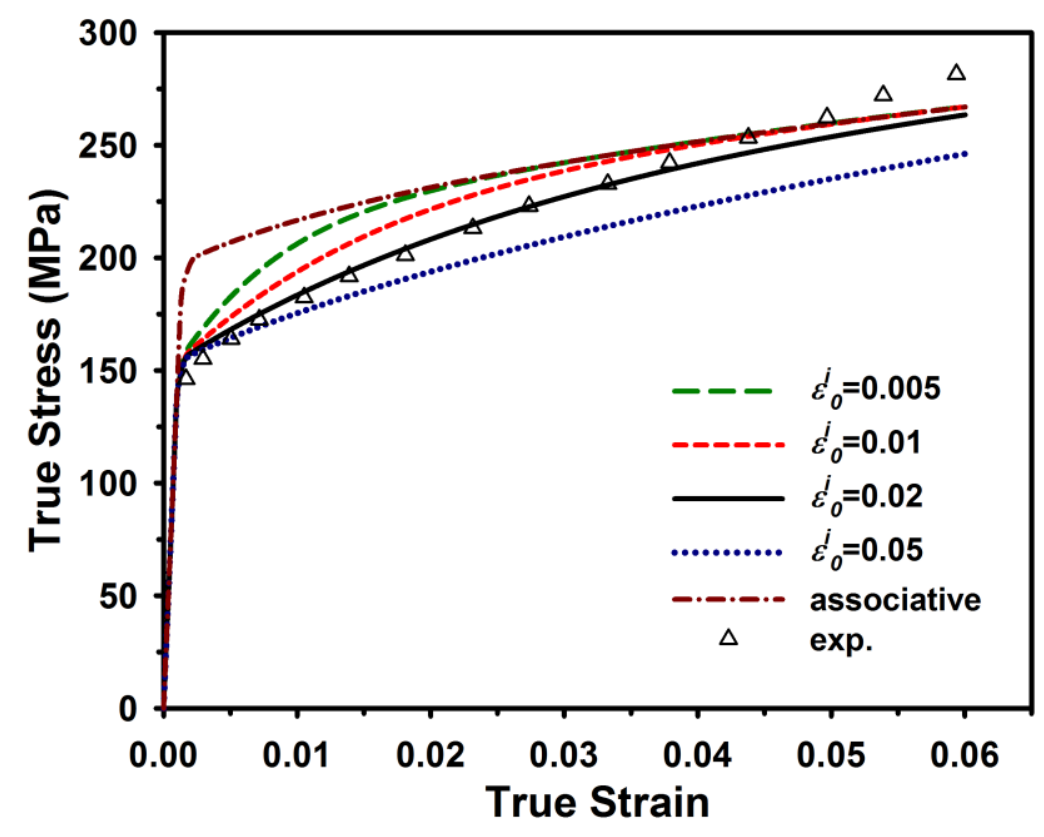

Figure 45. Parametric effect of $\varepsilon_{0}^{i}$ on the true stress-strain response of the material loaded in $\left[\begin{array}{lll}1 & 1 & 16\end{array}\right]$ orientation at $195 \mathrm{~K}$ compared to the associative flow behavior. Open symbols show the corresponding experimental data from [1].

As $\varepsilon_{0}^{i}$ is increased from 0.005 to 0.05 , the flow stress at a given strain decreases monotonically. The initial flow stress of the material with associative flow is higher than those with non-Schmid stresses. Note that relaxation of the stress-strain response to a quasiequilibrium state is observed at higher applied strains; this is more prominent for the material with lower $\varepsilon_{0}^{i}$. It might seem counter-intuitive that the material with higher non-Schmid stresses has a lower initial flow stress. Note that for glide to occur the driving stress, $\tau_{f}^{\alpha}$, must overcome the lattice frictional and obstacle resistance. Now, $\tau_{f}^{\alpha}$ has contributions from the resolved shear 
stress, $\tau^{\alpha}$, and the non-Schmid stresses (both components of the macroscopic stress state). When non-Schmid stresses are present, they complement $\tau^{\alpha}$ in overcoming the lattice resistance. However, for the case of associative flow, $\tau^{\alpha}$ must have a higher relative contribution to $\tau_{f}^{\alpha}$; $\tau^{\alpha}$, and hence the macroscopic stress has to increase at a higher rate to overcome the lattice resistance initially. As a result, inertial effects of the stress rate lead to a higher overstress with decreasing non-Schmid stress contribution. Viscous relaxation of the higher overstress ultimately leads to the stress-strain response approaching quasi-equilibrium during the later stages of deformation.

Comparison to experiments [1] shows that the material with $\varepsilon_{0}^{i}=0.02$ best fits the experimental stress-strain data, all other parameters remaining same. Accordingly, we have used $\varepsilon_{0}^{i}=0.02$ in all our simulations. Systematic experiments, for example, uniaxial loading of single crystals with different degrees of pre-strain, may be used to obtain a better estimate of this parameter (higher pre-strain would relate to lower non-Schmid contribution).

\subsubsection{Tension-compression asymmetry}

The constitutive model was used to predict the tension-compression (TC) asymmetry of single crystal Fe loaded in different orientations. This asymmetry measured in terms of the strength differential, $S D$, is given as [122]

$$
S D=\frac{\left(\sigma_{Y}^{t}-\sigma_{Y}^{c}\right)}{\left(\sigma_{Y}^{t}+\sigma_{Y}^{c}\right) / 2}
$$

where $\sigma_{Y}^{t}$ is the yield stress in tension, and $\sigma_{Y}^{c}$ is the yield stress in compression. The yield stress measured at $0.2 \%$ strain was used in these calculations. Figure 46 shows the variation of $S D$ with temperature for various loading orientations. A negative value of $S D$ indicates higher yield stress in compression.

As can be seen, the tension-compression asymmetry is highly orientation and temperature dependent. Loading orientations [ $\left[\begin{array}{lll}0 & 0 & 1\end{array}\right]$ and [ $\left[\begin{array}{lll}1 & 1 & 16\end{array}\right]$ are stereographically close by, hence the overlap in their TC asymmetry behavior, especially at $250 \mathrm{~K}$ and $295 \mathrm{~K}$. A similar qualitative, but higher, TC asymmetry is also seen for the [ [ $\left.\begin{array}{lll}1 & 1 & 1\end{array}\right]$ orientation. The yield stress is generally higher in compression, except for the [ $\left[\begin{array}{lll}0 & 1 & 1\end{array}\right]$ orientation at $143 \mathrm{~K}$. Similar predictions were also made for the TC asymmetry of single crystal Mo in Lim et al. [239] using a constitutive model with non-Schmid considerations. Also note that the TC asymmetry is generally lower at $250 \mathrm{~K}$ and $295 \mathrm{~K}$, though not zero. This again shows that TC asymmetry due to the non-Schmid effects is still present at room temperature. 
This asymmetry arises from activation of different slip systems in tension and compression. A comparison of the possible activated slip systems in tension versus compression is given in Weinberger et al. [238] for different loading orientations. There is no systematic experimental data available to compare to our model predictions. However, experiments $[253,254]$ have shown that tension-compression asymmetry is indeed prevalent in similar Fe-Si alloys, which have dominant non-Schmid effects.

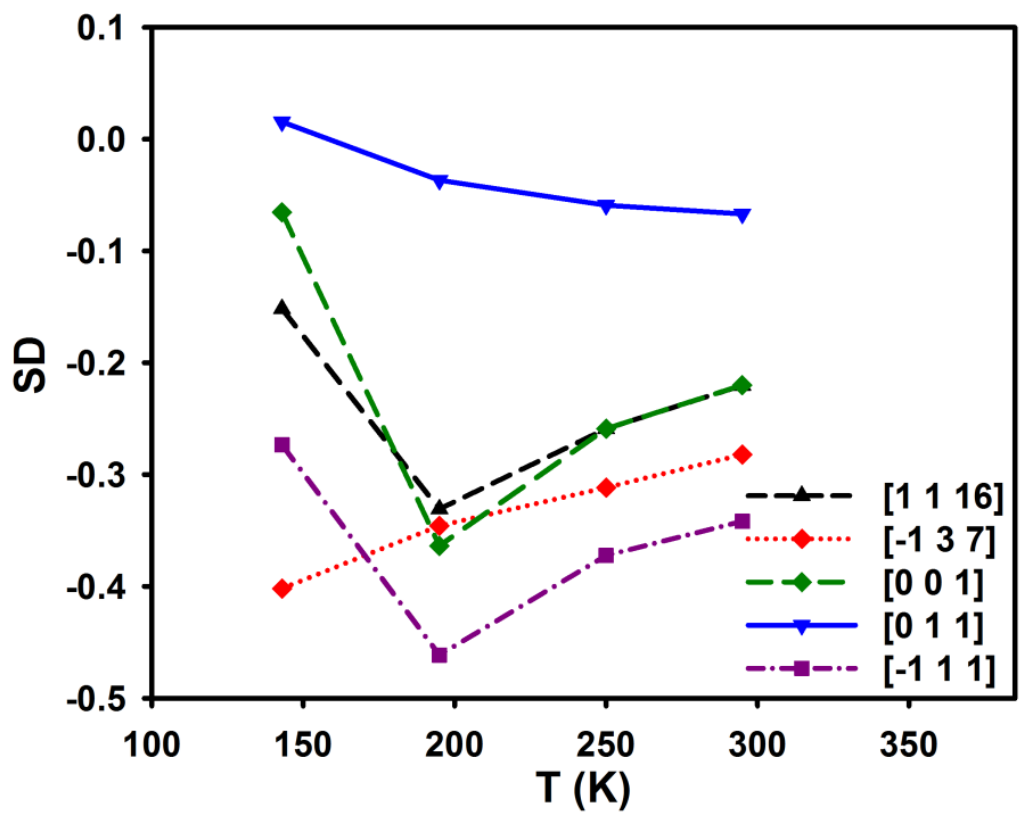

Figure 46. Variation of the strength differential, $S D$, with temperature for different loading orientations.

\subsubsection{Strain rate dependence}

The model was used to simulate the strain rate dependence of single crystal Fe loaded in the $\left[\begin{array}{lll}0 & 0 & 1\end{array}\right]$ orientation at $298 \mathrm{~K}$ by applying strain rates in the range $10^{-4}$ to $10^{0} \mathrm{~s}^{-1}$, with resulting stress-strain responses shown in Fig. 47 (a). As expected, the flow stress increases at higher strain rates. Figure 47 (b) plots the ratio of yield stress measured at a given strain rate to the corresponding yield stress at $10^{-4} \mathrm{~s}^{-1}$ for different strain rates. Simulation results of single crystal $\mathrm{Fe}$ at $298 \mathrm{~K}$ are compared with experimental data for polycrystalline $\mathrm{Fe}$ [5]; a test temperature of $288 \mathrm{~K}$ was used in the experiments. Though a one-to-one correspondence may not be expected, the simulated increase in yield stress with applied strain rate is in general agreement with experiments, at least up to a strain rate of $10^{-1} \mathrm{~s}^{-1}$. Beyond this, the simulations 
have a slightly higher rate sensitivity than the experiments. Note that experimental yield stress reported is for polycrystalline Fe. The pre-exponential factor, $\dot{\gamma}_{0}$, may be used to calibrate the strain rate dependence in terms of the yield and flow stress. In the present simulations, $\dot{\gamma}_{0}=5.0 \times 10^{4} \mathrm{~s}^{-1}$ was used. A higher value of $\dot{\gamma}_{0}$ would result in lower yield and flow stress.

(a)

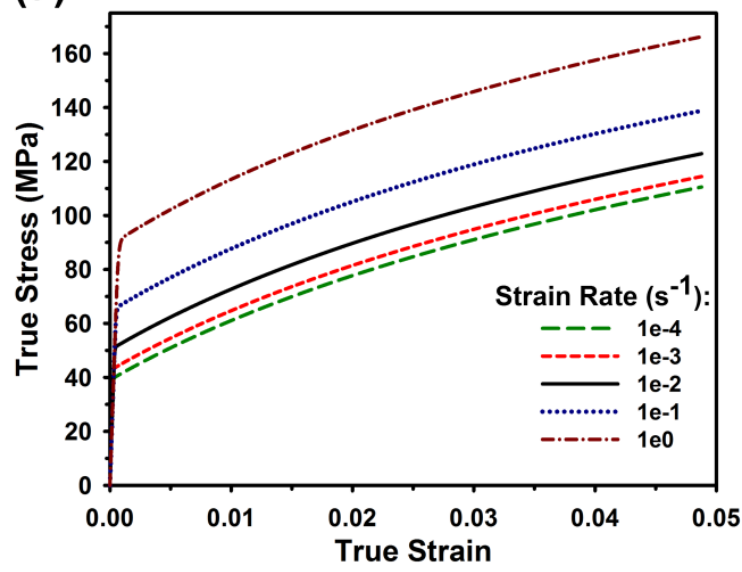

(b)

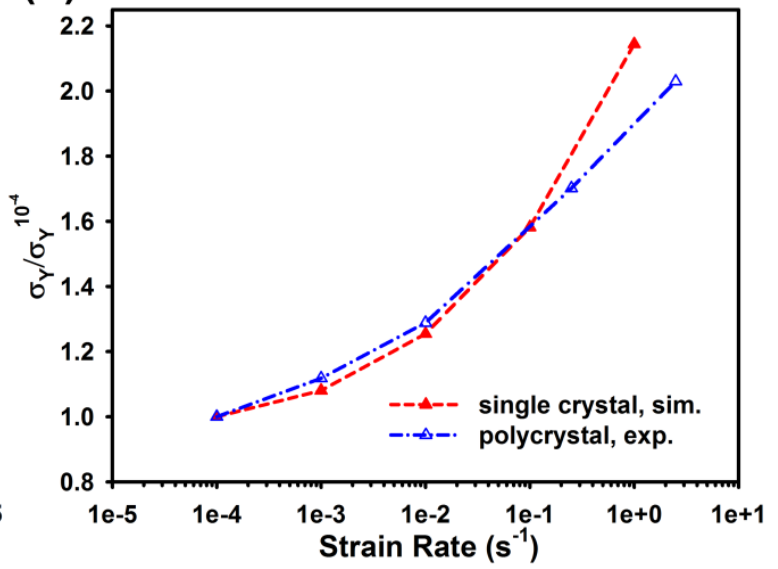

Figure 47. (a): Simulated strain rate sensitivity of single crystal Fe loaded in the [ $\left[\begin{array}{lll}0 & 0 & 1\end{array}\right]$ orientation at $298 \mathrm{~K}$. (b): Comparison of simulated $0.2 \%$ yield stress of single crystal Fe with experimentally measured lower yield stress of polycrystal $\mathrm{Fe}$ as a function of strain rate. Experimental data from [5].

\subsubsection{Implications for radiation damage}

The models discussed in this Chapter have so far not addressed any issues relating to radiation damage at low temperatures. This is in part due to the complicated nature of nonSchmid effects by itself, and also due to the lack of relevant experimental data for single crystal Fe to compare our model predictions. Moreover, delineating the irradiation effects from the nonSchmid effects presents an added challenge. While irradiation hardening and flow localization due interactions of dislocations with interstitial loops may be modeled using the constitutive equations developed in Chapters 2 and 3, interaction of dislocations with the irradiation-induced point defects is expected to affect their mobility and the unit processes of dislocation kink pair formation.

Dr. Ting Zhu and Sankar Narayanan at Georgia Tech are presently studying these interactions and their effect on the deformation mechanism using atomistic simulations. Based on personal communication, the following summarizes some of their recent findings. While the far-field dislocation-interstitial interaction is still governed by the elastic strain field of these defects, screw dislocation migration is controlled by cross-kink nucleation on secondary slip 
planes (as opposed to double kink nucleation) once the interstitial approaches the screw dislocation core. Further, the spatial orientation of interstitials around the dislocation core also affects the Peierls stress for dislocation kink nucleation. These effects would manifest themselves in a modified form of the enthalpy shape function for screw dislocation mobility in Eq. (6.8).

While the above mentioned studies are for the unit process of kink formation, we expect that the presence of a distribution of interstitials around a screw dislocation core would further hinder its glide by preventing core constriction on secondary slip planes. From a statistical viewpoint, these effects may be accounted in the present framework by modeling the nonSchmid coefficients as a function of the mean field interstitial concentration, i.e.,

$$
a_{j}^{T}=\left.a_{j}^{T}\right|_{0}+f_{j}\left(c_{i}\right)
$$

where $a_{j}^{T}$ is the $j^{\text {th }}$ non-Schmid coefficient, $\left.a_{j}^{T}\right|_{0}$ is the value of the corresponding non-Schmid coefficient in the absence of any irradiation (as modeled in the present simulations), and $f_{j}\left(c_{i}\right)$ is the functional dependence of the non-Schmid coefficient on the interstitial concentration, $c_{i}$, around the dislocation core. This last term needs to be determined from relevant atomistic calculations.

\subsection{Summary}

Constitutive equations have been developed for modeling non-Schmid effects in bcc-Fe at low and ambient temperatures. Constitutive equations for the decay of non-Schmid stresses as a function of inelastic strain are also developed. The non-Schmid parameters are used with an existing dislocation density crystal plasticity framework to simulate the mechanical behavior of single crystal Fe. The model is fit to the experimental hardening response at $298 \mathrm{~K}$. The yield stress and tension-compression asymmetry for different loading orientations and temperatures is also modeled. Model predictions are compared to available experimental data, including strain rate dependence of flow stress.

Temperature dependence of the non-Schmid parameters has not been examined previously. Further, previous continuum constitutive models generally use the non-Schmid parameters measured from atomistic simulations at $0 \mathrm{~K}$. This might not be physically accurate, as experiments suggest that the non-Schmid effects decay with increase in temperature. In this work, the non-Schmid parameters and their temperature dependence are derived entirely from available experimental data for Fe. The method used in this work may be used to estimate the temperature dependence of non-Schmid parameters for other bcc metals, such as Mo and Ta, as well. 


\section{CHAPTER 7}

\section{SUMMARY AND CONCLUSIONS}

\subsection{Summary and novel contributions}

\subsubsection{Constitutive model development}

A continuum crystal plasticity framework has been developed for modeling the mechanical behavior of irradiated bcc materials. The framework models the deformation behavior over a range of loading and radiation histories. Climb enhanced glide of dislocations is the principal inelastic deformation mechanism in this crystal plasticity framework. While the conventional glide component of deformation dominates during quasi-static loading, dislocation climb by stress-induced preferential absorption (SIPA) is more prevalent during creep loading of the irradiated materials.

A continuum damage framework has also been developed to model failure initiation at the interfaces of dislocation channels and grain boundaries (GBs) in irradiated bcc materials. Void nucleation and growth by condensation of supersaturated vacancies (generated during inelastic deformation) is the main failure mechanism in this damage framework.

Dislocation densities (mobile and immobile), size and number density of the irradiationinduced interstitial loops, and point defect concentrations are used as state variables in this crystal plasticity framework. The damage framework also uses the number density and size of voids as state variables, in addition to those used in the crystal plasticity framework.

Physically-based constitutive equations have been developed to model the evolution of these state variables by creation, exchange, interaction, and annihilation type mechanisms. Rate theory based equations for the evolution of point defects have also been integrated in this crystal plasticity framework. This is a unique feature of the present framework and has not been done previously. Rate theory calculations are usually performed for mean field material points and have not been correlated to the mechanical behavior previously. Radiation damage in metals is generally manifested in terms of point defect generation and their subsequent evolution. Correlation of the spatial point defect evolution to the deformation behavior is therefore fundamental to modeling radiation damage, more so in the case of irradiation creep. While irradiation hardening and subsequent flow localization can be modeled by simply considering the interstitial loops (in bcc metals), modeling irradiation creep requires consideration for the radiation-induced point defects, as well. Consideration of point defects also allows us to model 
void nucleation and growth by condensation of supersaturated vacancies generated during inelastic deformation.

In addition to modifying some of the existing models available in the literature, constitutive equations for the following physical mechanisms have been developed in this work:

- Inelastic deformation by climb enhanced glide of dislocations.

- Climb of mobile edge dislocations by absorbing point defect clusters and their transformation to interstitial loops.

- Growth of planar interstitial loops by absorbing point defects.

- Cross-slip of mobile screw dislocations.

- Annihilation of interstitial loops when they are within a critical capture radius of the interacting mobile dislocations, thus clearing pathways for easy dislocation glide.

- Temperature dependence of non-Schmid yield behavior.

- Decay of non-Schmid stresses with inelastic deformation.

- Diffusion-mediated growth of voids in a supersaturated field of vacancies under tensile hydrostatic stresses.

Integration of these models in the crystal plasticity framework not only provides a more accurate representation of the deformation behavior, but also allows us to delineate the effect of each of these separate mechanisms on the overall macroscopic mechanical response and microstructure evolution. Parametric study of these models also provides a guide for tailoring properties for the design of 'radiation-resistant' materials.

\subsubsection{Model application}

The framework has been used to model the following major features of inelastic deformation in irradiated bcc materials:

- Increase in yield stress due irradiation hardening and the initial dislocation evolution due to interaction with irradiation-induced defects.

- Flow localization along narrow dislocation channels due to annihilation of irradiationinduced interstitial loops by the interacting mobile dislocations.

- Failure initiation at the interfaces of these dislocation channels and GBs by void nucleation and growth processes.

- Irradiation creep deformation by dislocation climb enhanced glide.

- Temperature-dependent non-Schmid yield behavior in bcc metals.

Prior to this work, there have existed several constitutive crystal plasticity frameworks [12-15] to model the mechanical behavior of irradiated polycrystals. A distinct feature of our framework in comparison to these previous works is that we have explicitly modeled irradiation creep; none of these existing frameworks consider irradiation creep deformation. Moreover, microstructure evolution due to dislocation channel formation is not addressed explicitly in most 
of these frameworks. In contrast, we have modeled both the deformed microstructure evolution and the resulting mechanical behavior due to dislocation channel formation; correlating the deformed microstructure with the mechanical properties is essential to understanding the mechanics of these deformation processes.

Another novel contribution of this research is the void nucleation and growth based damage framework. While this framework was applied to model failure initiation at the dislocation channel interfaces in irradiated metals, the underlying physics of the framework is general enough for modeling void nucleation and growth mediated failure in virgin metals, as well.

Finally, constitutive modeling of the temperature dependence of non-Schmid stresses is another novel contribution of this research. While atomistically informed constitutive frameworks for modeling non-Schmid effects were developed previously (e.g. [229,238]), temperature dependence of the non-Schmid stresses was never studied systematically. Relevant findings of the present research pose the necessity for exploring these temperature effects in other bcc metals as well, in order to develop physically accurate constitutive models.

This coarse-grained constitutive framework is suited for modeling the mechanical behavior at the meso- and macroscales, while addressing the physics of the underlying deformation phenomena. Atomistic and dislocation dynamics simulations are indeed essential for understanding the defect evolution unit processes, but they are unfeasible for simulating polycrystalline ensembles at the scale of microns and over large timescales. Using information (of the physical deformation mechanisms) gained from these lower scale simulations, the present continuum framework may be used to model their effect on the macroscopic mechanical properties and microstructural evolution.

\subsection{Conclusions}

Based on application of the constitutive framework to model different loading conditions, the following major conclusions are drawn from the present research:

\section{Flow localization along dislocation channels}

- The constitutive framework is able to qualitatively capture the microstructural and mechanical aspects of inelastic flow localization due to dislocation channel formation in irradiated bcc materials.

- Flow localization in our simulations is a result of 'constitutive' material softening effects due to interstitial loop annihilation along preferred crystallographic planes, triggered by intergranular heterogeneity.

- Cross-slip promotes the formation of wider and more diffuse dislocation channels. 
- Maximum localized inelastic strain in the channels is a linear function of the radiation dpa dose for the same initial crystallographic orientation distribution of grains.

- There are two dominant length scales governing the localization phenomena. The dislocation channel width is governed by the mean free path of mobile dislocations, $l_{d}$, while the dislocation channel spacing is governed by the grain size.

\section{Failure initiation along dislocation channel interfaces and GBs ahead of a notch}

- The density of dislocation channels is much higher in the vicinity of a notch, as compared to the homogeneously deformed bulk.

- The void nucleation and growth based damage framework is able to model ductile 'zigzag' fracture and limited ductility 'channel' fracture in irradiated metals at elevated temperatures.

- Failure initiation is strongly sensitive to the interfacial energy change, $\Delta \Upsilon_{h e t}$, associated with heterogeneous void nucleation at the dislocation channel interfaces and GBs. This parameter may be used for calibrating our model predictions to experiments.

- The crack growth resistance of irradiated specimens is lower than that of the virgin specimens.

- The extent of localization and failure initiation is inhibited at higher strain rates.

\section{Irradiation creep deformation}

- Dislocation climb by stress-induced preferential absorption (SIPA) and subsequent dislocation glide is the primary irradiation creep mechanism in our framework. Using this mechanism, model predictions of creep rates and irradiation creep compliance are of the same order of magnitude as in experiments.

- The interstitial loop density was found to increase monotonically with ongoing radiation, while mean the loop size saturates following the initial transients.

- The in-situ radiation rate was measured in terms of $\mathrm{dpa} \cdot \mathrm{s}^{-1}$ using a phenomenological relationship that correlates the interstitial loop density with the radiation dpa damage. Using this correlation, the measured dpa rate was found to increase linearly with time. This asserts that the present framework is modeling irradiation creep correctly, as linear dpa rate is expected for a constant defect pair production rate.

- The creep strain rate decreases with increasing density of GBs and higher GB sink strengths. This is because point defect sinks inhibit the transfer of deformation across GBs. Resulting incompatibility of strain at the GBs may lead to intergranular failure.

\section{Non-Schmid yield behavior of bcc-iron at low and ambient temperatures}


- Using the atomistically measured $0 \mathrm{~K}$ non-Schmid coefficients for modeling the mechanical behavior of bcc metals at finite temperatures is physically inaccurate.

- The contribution of non-Schmid stresses required to constrict the screw dislocation cores (for them to glide) decreases with increasing temperature and increasing inelastic deformation.

- Based on fit to experimental data, the decay of non-Schmid coefficients w.r.t. temperature starts saturating at $\sim 250 \mathrm{~K}$ in bcc-iron.

- Non-Schmid yield behavior and resulting tension-compression asymmetry though reduced, is still non-negligible at room temperatures in bcc-iron.

\subsection{Recommendations for future work}

As with any model description, we have made various assumptions to simplify the constitutive equations and their numerical implementation. Most importantly, we have assumed a local material in all the model applications demonstrated in this research. While this might be a valid assumption in majority of the quasi-static loading conditions, point defect spatial diffusion may be present at the timescales of irradiation creep deformation. Point defect gradients are also expected to be non-negligible during slow strain rate in-reactor tension tests with concurrent irradiation. These defect gradients may be modeled in future work using the constitutive equations (5.3) and (5.4). In terms of numerical implementation, continuity of the point defect concentrations needs to be considered in a finite element framework in addition to the continuity of displacement. Alternatively, a numerical scheme may be developed where the point defect concentrations are mapped using a separate finite difference mesh, thus decoupling mechanical deformation and point defect diffusion.

As mentioned above, in-reactor tension tests may be simulated using this model framework in future work. This would be particularly interesting for exploring the effect of radiation dose on the dislocation channel formation behavior; Arsenlis et al. [44] showed that a threshold radiation dose exists below which channel formation does not take place. It would also be informative to perform combined loading simulations, e.g., in-reactor tensile loading followed by stress hold and study its effect on the irradiation creep rates.

While the framework has been applied to model different deformation histories, detailed parametric exploration (within the window of physical feasibility) of the various constitutive models has not been performed in the present research. This is especially important for quantifying the individual effect of each of these models on the measured mechanical properties and/or microstructural evolution characteristics, and the associated uncertainty.

Finally, validation of the constitutive models with relevant experiments, atomistic simulations, theoretical calculations, etc. is fundamental for applicability of this framework to practical engineering problems. For example, the interfacial energy change parameter, $\Delta \Upsilon_{h e t}$, 
associated with heterogeneous void nucleation may be estimated from atomistic simulations of the nucleation of voids at interfaces performed for different stress triaxialities and temperatures. This parameter would affect the accurate measurement of fracture toughness in our simulations. As another example, the material constant associated with the decay of non-Schmid stresses as a function of inelastic strain (in Eq. (6.13)) may be estimated by pre-straining iron single crystals and studying its effect on the yield stress and flow stress at different temperatures. The presence of interstitials near the screw dislocation core is also expected to affect the non-Schmid yield behavior. Relevant atomistic studies may be performed to determine the functional dependence of non-Schmid coefficients on the interstitial concentration (cf. Eq. (6.19)) in future work. 


\section{REFERENCES}

[1] W.A. Spitzig, A.S. Keh, Orientation and temperature dependence of slip in iron single crystals, Met. Trans. 1 (1970) 2751-2757.

[2] S.A. Maloy, M.R. James, G. Willcutt, W.F. Sommer, M. Sokolov, L.L. Snead, et al., The mechanical properties of 316L/304L stainless steels, Alloy 718 and Mod 9Cr-1Mo after irradiation in a spallation environment, J. Nucl. Mater. 296 (2001) 119-128.

[3] X. Jia, Y. Dai, The change of fracture toughness of martensitic steels after irradiation in SINQ target-3, J. Nucl. Mater. 356 (2006) 50-55.

[4] A.S. Keh, Work hardening and deformation sub-structure in iron single crystals deformed in tension at $298^{\circ} \mathrm{k}$, Philos. Mag. 12 (1965) 9-30.

[5] J. Harding, The effect of grain size and strain rate on the lower yield stress of pure iron at $288^{\circ} \mathrm{K}$, Acta Met. 17 (1969) 949-958.

[6] G.S. Was, Fundamentals of Radiation Material Science: Metals and Alloys, Springer, Berlin, 2007.

[7] S.J. Zinkle, G.E. Lucas, Deformation and fracture mechanisms in irradiated FCC and BCC metals, Fusion Mater. Semi-Annu. Prog. Rep. Period End. June 302003 DOEER-031334 Oak Ridge Natl. Lab. (2003) 101-125.

[8] S.J. Zinkle, G.S. Was, Materials challenges in nuclear energy, Acta Mater. 61 (2013) 735758.

[9] F.. Garner, M.. Toloczko, B.. Sencer, Comparison of swelling and irradiation creep behavior of fcc-austenitic and bcc-ferritic/martensitic alloys at high neutron exposure, J. Nucl. Mater. 276 (2000) 123-142.

[10] L.K. Mansur, A.F. Rowcliffe, R.K. Nanstad, S.J. Zinkle, W.R. Corwin, R.E. Stoller, Materials needs for fusion, Generation IV fission reactors and spallation neutron sources similarities and differences, J. Nucl. Mater. 329-333 (2004) 166-172.

[11] O. Anderoglu, T.S. Byun, M. Toloczko, S.A. Maloy, Mechanical Performance of Ferritic Martensitic Steels for High Dose Applications in Advanced Nuclear Reactors, Met. Mater. Trans. 44 (2013) 70-83.

[12] A. Arsenlis, B.D. Wirth, M. Rhee, Dislocation density-based constitutive model for the mechanical behaviour of irradiated Cu, Philos. Mag. 84 (2004) 3617-3635.

[13] S. Krishna, A. Zamiri, S. De, Dislocation and defect density-based micromechanical modeling of the mechanical behavior of fcc metals under neutron irradiation, Philos. Mag. 90 (2010) 4013-4025.

[14] D. Li, H. Zbib, H. Garmestani, X. Sun, M. Khaleel, Modeling of irradiation hardening of polycrystalline materials, Comput. Mater. Sci. 50 (2011) 2496-2501.

[15] N.R. Barton, A. Arsenlis, J. Marian, A polycrystal plasticity model of strain localization in irradiated iron, J. Mech. Phys. Solids. 61 (2013) 341-351.

[16] A. Patra, D.L. McDowell, Crystal plasticity-based constitutive modelling of irradiated bcc structures, Philos. Mag. 92 (2012) 861-887. 
[17] G.R. Odette, B.D. Wirth, D.J. Bacon, N.M. Ghoniem, Multiscale-multiphysics modeling of radiation-damaged materials: embrittlement of pressure-vessel steels, MRS Bull. 26 (2001) 176-181.

[18] G.R. Odette, G.E. Lucas, Embrittlement of nuclear reactor pressure vessels, JOM J. Miner. Met. Mater. Soc. 53 (2001) 18-22.

[19] B.D. Wirth, G.R. Odette, D. Maroudas, G.E. Lucas, Energetics of formation and migration of self-interstitials and self-interstitial clusters in [alpha]-iron, J. Nucl. Mater. 244 (1997) $185-194$.

[20] J. Marian, B.D. Wirth, J.M. Perlado, Mechanism of formation and growth of <100> interstitial loops in ferritic materials, Phys. Rev. Lett. 88 (2002) 255507.

[21] B.D. Wirth, G.R. Odette, D. Maroudas, G.E. Lucas, Dislocation loop structure, energy and mobility of self-interstitial atom clusters in bcc iron, J. Nucl. Mater. 276 (2000) 33-40.

[22] T. Diaz de la Rubia, H.M. Zbib, T.A. Khraishi, B.D. Wirth, M. Victoria, M.J. Caturla, Multiscale modelling of plastic flow localization in irradiated materials, Nature. 406 (2000) 871-874.

[23] B.D. Wirth, V. Bulatov, T. Diaz de la Rubia, Atomistic simulation of stacking fault tetrahedra formation in Cu, J. Nucl. Mater. 283-287 (2000) 773-777.

[24] S.J. Zinkle, Y. Matsukawa, Observation and analysis of defect cluster production and interactions with dislocations, J. Nucl. Mater. 329-333 (2004) 88-96.

[25] S.J. Zinkle, P.J. Maziasz, R.E. Stoller, Dose dependence of the microstructural evolution in neutron-irradiated austenitic stainless steel, J. Nucl. Mater. 206 (1993) 266-286.

[26] M.I. Luppo, C. Bailat, R. Schäublin, M. Victoria, Tensile properties and microstructure of $590 \mathrm{MeV}$ proton-irradiated pure Fe and a Fe-Cr alloy, J. Nucl. Mater. 283-287 (2000) 483487.

[27] N.M. Ghoniem, G.L. Kulcinski, The effect of pulsed irradiation on the swelling of 316 stainless steel in fusion reactors, Nucl. Eng. Des. 52 (1979) 111-125.

[28] L.K. Mansur, T.C. Reiley, Irradiation creep by dislocation glide enabled by preferred absorption of point defects -- theory and experiment, J. Nucl. Mater. 90 (1980) 60-67.

[29] H. Rauh, R. Bullough, J.R. Matthews, The dislocation bias during irradiation: Effects of climb motion and rate limitation, Philos. Mag. 65 (1992) 53-70.

[30] H.J. Frost, M.F. Ashby, Deformation-Mechanism Maps, Permagon Press, Oxford, 1982.

[31] J. Weertman, Theory of steady-state creep based on dislocation climb, J. Appl. Phys. 26 (1955) 1213-1217.

[32] R. Bullough, M.H. Wood, Mechanisms of radiation induced creep and growth, J. Nucl. Mater. 90 (1980) 1-21.

[33] J.R. Matthews, M.W. Finnis, Irradiation creep models -- an overview, J. Nucl. Mater. 159 (1988) 257-285.

[34] A.D. Brailsford, R. Bullough, Irradiation creep due to the growth of interstitial loops, Philos. Mag. 27 (1973) 49. 
[35] P.T. Heald, M.V. Speight, Steady-state irradiation creep, Philos. Mag. 29 (1974) 10751080.

[36] R. Bullough, J.R. Willis, The stress-induced point defect-dislocation interaction and its relevance to irradiation creep, Philos. Mag. 31 (1975) 855-861.

[37] R. Bullough, M.R. Hayns, Irradiation-creep due to point defect absorption, J. Nucl. Mater. 57 (1975) 348-352.

[38] L.K. Mansur, Irradiation creep by climb-enabled glide of dislocations resulting from preferred absorption of point defects, Philos. Mag. 39 (1979) 497-506.

[39] N.M. Ghoniem, J.R. Matthews, R.J. Amodeo, A dislocation model for creep in engineering materials, Res Mech. 29 (1990) 197-219.

[40] G.R. Odette, D. Frey, Development of mechanical property correlation methodology for fusion environments, J. Nucl. Mater. 85-86 (1979) 817-822.

[41] G.E. Lucas, The evolution of mechanical property change in irradiated austenitic stainless steels, J. Nucl. Mater. 206 (1993) 287-305.

[42] B.N. Singh, A.J.E. Foreman, H. Trinkaus, Radiation hardening revisited: role of intracascade clustering, J. Nucl. Mater. 249 (1997) 103-115.

[43] N.M. Ghoniem, B.N. Singh, L.Z. Sun, T. Díaz de la Rubia, Interaction and accumulation of glissile defect clusters near dislocations, J. Nucl. Mater. 276 (2000) 166-177.

[44] A. Arsenlis, M. Rhee, G. Hommes, R. Cook, J. Marian, A dislocation dynamics study of the transition from homogeneous to heterogeneous deformation in irradiated body-centered cubic iron, Acta Mater. 60 (2012) 3748-3757.

[45] Y. Estrin, L.P. Kubin, Local strain hardening and nonuniformity of plastic deformation, Acta Met. 34 (1986) 2455-2464.

[46] R.J. Asaro, J.R. Rice, Strain localization in ductile single crystals, J. Mech. Phys. Solids. 25 (1977) 309-338.

[47] R.J. Asaro, Micromechanics of crystals and polycrystals, Adv. Appl. Mech. 23 (1983) 1115.

[48] M. Kothari, L. Anand, Elasto-viscoplastic constitutive equations for polycrystalline metals: Application to tantalum, J. Mech. Phys. Solids. 46 (1998) 51-67.

[49] U.F. Kocks, A.S. Argon, M.F. Ashby, Thermodynamics and kinetics of slip, Permagon Press, 1975.

[50] C. Deo, C. Tomé, R. Lebensohn, S. Maloy, Modeling and simulation of irradiation hardening in structural ferritic steels for advanced nuclear reactors, J. Nucl. Mater. 377 (2008) 136-140.

[51] D.L. McDowell, Viscoplasticity of heterogeneous metallic materials, Mater. Sci. Eng. R Reports. 62 (2008) 67-123.

[52] M.S. Duesbery, V. Vitek, D.K. Bowen, The Effect of Shear Stress on the Screw Dislocation Core Structure in Body-Centred Cubic Lattices, Proc. R. Soc. Lond. Math. Phys. Sci. 332 (1973) 85-111. 
[53] M.S. Duesbery, On Non-Glide Stresses and Their Influence on the Screw Dislocation Core in Body-Centred Cubic Metals. I. The Peierls Stress, Proc. R. Soc. Lond. Ser. Math. Phys. Sci. 392 (1984) 145-173.

[54] Q. Qin, J.L. Bassani, Non-schmid yield behavior in single crystals, J. Mech. Phys. Solids. 40 (1992) 813-833.

[55] Q. Qin, J.L. Bassani, Non-associated plastic flow in single crystals, J. Mech. Phys. Solids. 40 (1992) 835-862.

[56] V. Racherla, J.L. Bassani, Strain burst phenomena in the necking of a sheet that deforms by non-associated plastic flow, Model. Simul. Mater. Sci. Eng. 15 (2007) S297-S311.

[57] V. Vitek, V. Paidar, Chapter 87 Non-planar dislocation cores: a ubiquitous phenomenon affecting mechanical properties of crystalline materials, in: J.P. Hirth (Ed.), Dislocations Solids Tribute FRN Nabarro, Elsevier, 2008: pp. 439-514.

[58] M. Peach, J.S. Koehler, The Forces Exerted on Dislocations and the Stress Fields Produced by Them, Phys. Rev. 80 (1950) 436.

[59] E. Orowan, Problems of plastic gliding, Proc. Phys. Soc. 52 (1940) 8-22.

[60] R. Sizmann, The effect of radiation upon diffusion in metals, J. Nucl. Mater. 69-70 (1978) 386-412.

[61] A.D. Brailsford, R. Bullough, The rate theory of swelling due to void growth in irradiated metals, J. Nucl. Mater. 44 (1972) 121-135.

[62] R.E. Stoller, G.R. Odette, Microstructural evolution in an austenitic stainless steel fusion reactor first wall, J. Nucl. Mater. 141-143 (1986) 647-653.

[63] R.E. Stoller, Modeling dislocation evolution in irradiated alloys, Met. Trans. 21 (1990) 1829-1837.

[64] A.A. Semenov, C.H. Woo, Stochastic effects on dislocation structure development under cascade-damage irradiation, Appl. Phys. Mater. Sci. Process. 67 (1998) 193-207.

[65] U. Essmann, H. Mughrabi, Annihilation of dislocations during tensile and cyclic deformation and limits of dislocation densities, Philos. Mag. 40 (1979) 731-756.

[66] J.P. Hirth, J. Lothe, Theory of dislocations, 2nd ed., John Wiley and Sons, New York, 1982.

[67] M. Rhee, H.M. Zbib, J.P. Hirth, H. Huang, T.D. de la Rubia, Models for long-/short-range interactions and cross slip in 3D dislocation simulation of BCC single crystals, Model. Simul. Mater. Sci. Eng. 6 (1998) 467-492.

[68] A. Arsenlis, D.M. Parks, Modeling the evolution of crystallographic dislocation density in crystal plasticity, J. Mech. Phys. Solids. 50 (2002) 1979-2009.

[69] U.F. Kocks, A statistical theory of flow stress and work-hardening, Philos. Mag. 13 (1966) 541-566.

[70] R.A. Austin, D.L. McDowell, A dislocation-based constitutive model for viscoplastic deformation of fcc metals at very high strain rates, Int. J. Plast. 27 (2011) 1-24. 
[71] Y. Estrin, Dislocation density-related constitutive modeling, in: Unified Const. Laws Plast. Deform., Academic Press, San Diego, 1996: pp. 69-106.

[72] Y. Estrin, H. Mecking, A unified phenomenological description of work hardening and creep based on one-parameter models, Acta Met. 32 (1984) 57-70.

[73] N. Hashimoto, S.J. Zinkle, R.L. Klueh, A.F. Rowcliffe, K. Shiba, Deformation Mechanisms in Ferritic/Martensitic Steels Irradiated in HFIR, Mater. Res. Soc. Symp. Proc. 650 (2001) R1.10.

[74] K. Farrell, T.S. Byun, N. Hashimoto, Deformation mode maps for tensile deformation of neutron-irradiated structural alloys, J. Nucl. Mater. 335 (2004) 471-486.

[75] S.J. Zinkle, B.N. Singh, Microstructure of neutron-irradiated iron before and after tensile deformation, J. Nucl. Mater. 351 (2006) 269-284.

[76] M. Ashrafi-Nik, Thermo hydraulic optimisation of the EURISOL DS target, EURISOLDS/TASK2/TN-02-25-2008-0016, 2006.

[77] V.M. Schastlivtsev, D.P. Rodionov, Y.V. Khlebnikova, I.L. Yakovleva, Peculiarity of structure and crystallography of plastic deformation of lath martensite in structural steels, Mater. Sci. Eng. 273-275 (1999) 437-442.

[78] R.A. Johnson, D.J. Oh, Analytic embedded atom method model for bcc metals, J. Mater. Res. 4 (1989) 1195-1201.

[79] M.F. Ashby, Mechanisms of deformation and fracture, Adv. Appl. Mech. 23 (1983) 117177.

[80] T. Suzuki, H. Koizumi, H.O.K. Kirchner, Plastic flow stress of b.c.c. transition metals and the Peierls potential, Acta Met. Mater. 43 (1995) 2177-2187.

[81] S. Queyreau, G. Monnet, B. Devincre, Slip systems interactions in [alpha]-iron determined by dislocation dynamics simulations, Int. J. Plast. 25 (2009) 361-377.

[82] M.I. Mendelev, Y. Mishin, Molecular dynamics study of self-diffusion in bcc Fe, Phys. Rev. B. 80 (2009) 144111.

[83] R.E. Stoller, S.I. Golubov, C. Domain, C.S. Becquart, Mean field rate theory and object kinetic Monte Carlo: A comparison of kinetic models, J. Nucl. Mater. 382 (2008) 77-90.

[84] J. Marian, B.D. Wirth, R. Schäublin, J.M. Perlado, T. Díaz de la Rubia, <1 0 0>-Loop characterization in [alpha]-Fe: comparison between experiments and modeling, J. Nucl. Mater. 307-311 (2002) 871-875.

[85] L.P. Evers, W.A.M. Brekelmans, M.G.D. Geers, Scale dependent crystal plasticity framework with dislocation density and grain boundary effects, Int. J. Solids Struct. 41 (2004) 5209-5230.

[86] ABAQUS, Simulia, Dassault Systemes, Providence, RI, 2007.

[87] O. van der Sluis, P.J.G. Schreurs, W.A.M. Brekelmans, H.E.H. Meijer, Overall behaviour of heterogeneous elastoviscoplastic materials: effect of microstructural modelling, Mech. Mater. 32 (2000) 449-462. 
[88] K. Sawada, K. Miyahara, H. Kushima, K. Kimura, S. Matsuoka, Contribution of Microstructural Factors to Hardness Change During Creep Exposure in Mod.9Cr-1Mo Steel, ISIJ Int. 45 (2005) 1934-1939.

[89] M. Matijasevic, E. Lucon, A. Almazouzi, Behavior of ferritic/martensitic steels after nirradiation at 200 and $300{ }^{\circ} \mathrm{C}$, J. Nucl. Mater. 377 (2008) 101-108.

[90] H. Magnusson, R. Sandström, Creep Strain Modeling of 9 to 12 Pct Cr Steels Based on Microstructure Evolution, Met. Mater. Trans. 38 (2007) 2033-2039.

[91] A. Patra, D.L. McDowell, Continuum modeling of localized deformation in irradiated bcc materials, J. Nucl. Mater. 432 (2013) 414-427.

[92] R.L. Klueh, J.M. Vitek, Fluence and helium effects on the tensile properties of ferritic steels at low temperatures, J. Nucl. Mater. 161 (1989) 13-23.

[93] A.F. Rowcliffe, J.P. Robertson, R.L. Klueh, K. Shiba, D.J. Alexander, M.L. Grossbeck, et al., Fracture toughness and tensile behavior of ferritic-martensitic steels irradiated at low temperatures, J. Nucl. Mater. 258-263 (1998) 1275-1279.

[94] P. Spätig, R. Schäublin, S. Gyger, M. Victoria, Evolution of the mechanical properties of the F82H ferritic/martensitic steel after $590 \mathrm{MeV}$ proton irradiation, J. Nucl. Mater. 258263 (1998) 1345-1349.

[95] S.A. Maloy, M.B. Toloczko, K.J. McClellan, T. Romero, Y. Kohno, F.A. Garner, et al., The effects of fast reactor irradiation conditions on the tensile properties of two ferritic/martensitic steels, J. Nucl. Mater. 356 (2006) 62-69.

[96] R.P. Tucker, M.S. Wechsler, S.M. Ohr, Dislocation Channeling in Neutron-Irradiated Niobium, J. Appl. Phys. 40 (1969) 400-408.

[97] D.J. Edwards, B.N. Singh, Evolution of cleared channels in neutron-irradiated pure copper as a function of tensile strain, J. Nucl. Mater. 329-333, Part B (2004) 1072-1077.

[98] D.J. Edwards, B.N. Singh, J.B. Bilde-Sørensen, Initiation and propagation of cleared channels in neutron-irradiated pure copper and a precipitation hardened $\mathrm{CuCrZr}$ alloy, J. Nucl. Mater. 342 (2005) 164-178.

[99] Z. Jiao, J.T. Busby, G.S. Was, Deformation microstructure of proton-irradiated stainless steels, J. Nucl. Mater. 361 (2007) 218-227.

[100] B.L. Eyre, A.F. Bartlett, An electron microscope study of neutron irradiation damage in alpha-iron, Philos. Mag. 12 (1965) 261-272.

[101] M. Victoria, N. Baluc, C. Bailat, Y. Dai, M.I. Luppo, R. Schäublin, et al., The microstructure and associated tensile properties of irradiated fcc and bcc metals, J. Nucl. Mater. 276 (2000) 114-122.

[102] F.A. Smidt, B. Mastel, Some observations of dislocation channelling in irradiated iron, Philos. Mag. 20 (1969) 651-656.

[103] E.H. Lee, T.S. Byun, J.D. Hunn, M.H. Yoo, K. Farrell, L.K. Mansur, On the origin of deformation microstructures in austenitic stainless steel: part I- microstructures, Acta Mater. 49 (2001) 3269-3276. 
[104] J.S. Robach, I.M. Robertson, B.D. Wirth, A. Arsenlis, In-situ transmission electron microscopy observations and molecular dynamics simulations of dislocation-defect interactions in ion-irradiated copper, Philos. Mag. 83 (2003) 955-967.

[105] Z. Jiao, G.S. Was, The role of irradiated microstructure in the localized deformation of austenitic stainless steels, J. Nucl. Mater. 407 (2010) 34-43.

[106] B.L. Eyre, Direct observations of neutron irradiation damage in [alpha]-iron, Philos. Mag. 7 (1962) 2107-2113.

[107] P.M. Rice, S.J. Zinkle, Temperature dependence of the radiation damage microstructure in V-4Cr-4Ti neutron irradiated to low dose, J. Nucl. Mater. 258-263 (1998) 1414-1419.

[108] A. Luft, Microstructural processes of plastic instabilities in strengthened metals, Prog. Mater. Sci. 35 (1991) 97-204.

[109] R. Chaouadi, Effect of irradiation-induced plastic flow localization on ductile crack resistance behavior of a 9\%Cr tempered martensitic steel, J. Nucl. Mater. 372 (2008) 379390.

[110] J.S. Robach, I.M. Robertson, H.-J. Lee, B.D. Wirth, Dynamic observations and atomistic simulations of dislocation-defect interactions in rapidly quenched copper and gold, Acta Mater. 54 (2006) 1679-1690.

[111] B.D. Wirth, V.V. Bulatov, T.D. de la Rubia, Dislocation-Stacking Fault Tetrahedron Interactions in Cu, J. Eng. Mater. Technol. 124 (2002) 329-334.

[112] Y.N. Osetsky, R.E. Stoller, D. Rodney, D.J. Bacon, Atomic-scale details of dislocationstacking fault tetrahedra interaction, Mater. Sci. Eng. 400-401 (2005) 370-373.

[113] Y. Matsukawa, Y.N. Osetsky, R.E. Stoller, S.J. Zinkle, Destruction processes of large stacking fault tetrahedra induced by direct interaction with gliding dislocations, J. Nucl. Mater. 351 (2006) 285-294.

[114] D. Rodney, Atomic modeling of irradiation-induced hardening, Comptes Rendus Phys. 9 (2008) 418-426.

[115] H.-J. Lee, B.D. Wirth, Molecular dynamics simulation of the interaction between a mixed dislocation and a stacking fault tetrahedron, Philos. Mag. 89 (2009) 821-841.

[116] H.J. Lee, J.H. Shim, B.D. Wirth, Molecular dynamics simulation of screw dislocation interaction with stacking fault tetrahedron in face-centered cubic $\mathrm{Cu}$, J. Mater. Res. 22 (2007) 2758-2769.

[117] T. Nogaret, D. Rodney, M. Fivel, C. Robertson, Clear band formation simulated by dislocation dynamics: Role of helical turns and pile-ups, J. Nucl. Mater. 380 (2008) 22-29.

[118] T. Nogaret, D. Rodney, M. Fivel, C. Robertson, Clear Band formation simulated by dislocation dynamics, Probl. At. Sci. Technol. 4 (2009) 97-108.

[119] J.L. Bassani, K. Ito, V. Vitek, Complex macroscopic plastic flow arising from nonplanar dislocation core structures, Mater. Sci. Eng. 319-321 (2001) 97-101.

[120] A. Seeger, Why anomalous slip in body-centred cubic metals?, Mater. Sci. Eng. 319-321 (2001) 254-260. 
[121] R. Gröger, V. Vitek, Multiscale modeling of plastic deformation of molybdenum and tungsten. III. Effects of temperature and plastic strain rate, Acta Mater. 56 (2008) 54265439.

[122] J.L. Bassani, V. Racherla, From non-planar dislocation cores to non-associated plasticity and strain bursts, Prog. Mater. Sci. 56 (2011) 852-863.

[123] D.S. Gelles, R.E. Schäublin, Post-irradiation deformation in a Fe-9\%Cr alloy, Mater. Sci. Eng. 309-310 (2001) 82-86.

[124] D. Terentyev, D.J. Bacon, Y.N. Osetsky, Reactions between a $1 / 2\langle 111\rangle$ screw dislocation and $\langle 100\rangle$ interstitial dislocation loops in alpha-iron modelled at atomic scale, Philos. Mag. 90 (2010) 1019-1033.

[125] A. Nomoto, N. Soneda, A. Takahashi, S. Ishino, Interaction analysis between edge dislocation and self interstitial type dislocation loop in BCC iron using molecular dynamics, Mater. Trans. 46 (2005) 463-468.

[126] D.J. Bacon, Y.N. Osetsky, Z. Rong, Computer simulation of reactions between an edge dislocation and glissile self-interstitial clusters in iron, Philos. Mag. 86 (2006) 3921-3936.

[127] L.Z. Sun, N.M. Ghoniem, S.-H. Tong, B.N. Singh, 3D dislocation dynamics study of plastic instability in irradiated copper, J. Nucl. Mater. 283-287, Part 2 (2000) 741-745.

[128] N.M. Ghoniem, S.-H. Tong, B.N. Singh, L.Z. Sun, On dislocation interaction with radiation-induced defect clusters and plastic flow localization in fec metals, Philos. Mag. 81 (2001) 2743-2764.

[129] T.A. Khraishi, H.M. Zbib, T.D. de la Rubia, M. Victoria, Localized deformation and hardening in irradiated metals: Three-dimensional discrete dislocation dynamics simulations, Met. Mater. Trans. B. 33 (2002) 285-296.

[130] D. Rodney, G. Martin, Y. Bréchet, Irradiation hardening by interstitial loops: atomistic study and micromechanical model, Mater. Sci. Eng. 309-310 (2001) 198-202.

[131] B.N. Singh, X. Huang, S. Tahtinen, P. Moilanen, P. Jacquet, J. Dekeyser, Final report on in-reactor tensile deformation of pure iron and Fe-Cr alloy, Riso National Laboratory, Technical University of Denmark, Roskilde, Denmark, 2007.

[132] B.N. Singh, D.J. Edwards, S. Tahtinen, P. Moilanen, P. Jacquet, J. Dekeyser, Final report on in-reactor tensile tests on OFHC-copper and $\mathrm{CuCrZr}$ alloy, Riso National Laboratory, Technical University of Denmark, Roskilde, Denmark, 2004.

[133] R. LeSar, J.M. Rickman, Coarse Graining of Dislocation Structure and Dynamics, in: D. Raabe, F. Roters, F. Barlat, L.-Q. Chen (Eds.), Contin. Scale Simul. Eng. Mater., WileyVCH Verlag GmbH \& Co. KGaA, 2005: pp. 429-444.

[134] J.V. Sharp, Deformation of neutron-irradiated copper single crystals, Philos. Mag. 16 (1967) 77-96.

[135] V.V. Bulatov, M. Tang, H.M. Zbib, Crystal Plasticity from Dislocation Dynamics, MRS Bull. 26 (2001) 191-195.

[136] M. Zhang, F. Bridier, P. Villechaise, J. Mendez, D.L. McDowell, Simulation of slip band evolution in duplex Ti-6Al-4V, Acta Mater. 58 (2010) 1087-1096. 
[137] T.S. Byun, N. Hashimoto, K. Farrell, E.H. Lee, Characteristics of microscopic strain localization in irradiated 316 stainless steels and pure vanadium, J. Nucl. Mater. 349 (2006) 251-264.

[138] M. Briceno, J. Fenske, M. Dadfarnia, P. Sofronis, I.M. Robertson, Effect of ion irradiation-produced defects on the mobility of dislocations in 304 stainless steel, J. Nucl. Mater. 409 (2011) 18-26.

[139] J.V. Sharp, Correlation between cleared channels and surface slip steps in neutron irradiated copper crystals, Radiat. Eff. 14 (1972) 71-75.

[140] J.L. Brimhall, B. Mastel, Deformation structure of neutron-irradiated copper-aluminum alloy, Appl. Phys. Lett. 9 (1966) 127-128.

[141] J.V. Sharp, Deformation of neutron irradiated copper alloys, Acta Met. 22 (1974) 449457.

[142] H.M. Zbib, E.C. Aifantis, On the gradient-dependent theory of plasticity and shear banding, Acta Mech. 92 (1992) 209-225.

[143] E.C. Aifantis, On the microstructural origin of certain inelastic models, J. Eng. Mater. Technol. 106 (1984) 326-330.

[144] H.M. Zbib, E.C. Aifantis, A Gradient-Dependent Flow Theory of Plasticity: Application to Metal and Soil Instabilities, Appl. Mech. Rev. 42 (1989) S295.

[145] H.. Zbib, E.. Aifantis, Size effects and length scales in gradient plasticity and dislocation dynamics, Scr. Mater. 48 (2003) 155-160.

[146] J. Pontes, D. Walgraef, E.C. Aifantis, On dislocation patterning: Multiple slip effects in the rate equation approach, Int. J. Plast. 22 (2006) 1486-1505.

[147] D. Walgraef, E.C. Aifantis, On certain problems of deformation-induced material instabilities, Int. J. Eng. Sci. 59 (2012) 140-155.

[148] G.R. Odette, M.Y. He, E.G. Donahue, P. Spätig, T. Yamamoto, Modeling the multiscale mechanics of flow localization-ductility loss in irradiation damaged bec alloys, J. Nucl. Mater. 307-311 (2002) 171-178.

[149] M. Sauzay, K. Bavard, W. Karlsen, TEM observations and finite element modelling of channel deformation in pre-irradiated austenitic stainless steels - Interactions with free surfaces and grain boundaries, J. Nucl. Mater. 406 (2010) 152-165.

[150] P. Evrard, M. Sauzay, Modelling of the effect of dislocation channel on intergranular microcrack nucleation in pre-irradiated austenitic stainless steels during low strain rate tensile loading, J. Nucl. Mater. 405 (2010) 83-94.

[151] G. Po, N. Ghoniem, Continuum Modeling of Plastic Flow Localization in Irradiated fcc Metals, J. Nucl. Mater. doi: 10.1016/j.jnucmat.2012.10.039 (2012).

[152] E.A. Little, Fracture mechanics evaluations of neutron irradiated Type 321 austenitic steel, J. Nucl. Mater. 139 (1986) 261-276. 
[153] R. Fish, C. Hunter, Tensile Properties of Fast Reactor Irradiated Type 304 Stainless Steel, in: F. Shober (Ed.), Irradiat. Eff. Microstruct. Prop. Met., ASTM International, West Conshohocken, PA, 1976: pp. 119-138.

[154] E. West, M. McMurtrey, Z. Jiao, G. Was, Role of Localized Deformation in IrradiationAssisted Stress Corrosion Cracking Initiation, Met. Mater. Trans. 43 (2012) 136-146.

[155] M.D. McMurtrey, G.S. Was, L. Patrick, D. Farkas, Relationship between localized strain and irradiation assisted stress corrosion cracking in an austenitic alloy, Mater. Sci. Eng. 528 (2011) 3730-3740.

[156] J. Henry, X. Averty, Y. Dai, P. Lamagnère, J.P. Pizzanelli, J.J. Espinas, et al., Tensile properties of $9 \mathrm{Cr}-1 \mathrm{Mo}$ martensitic steel irradiated with high energy protons and neutrons, J. Nucl. Mater. 318 (2003) 215-227.

[157] D.J. Michel, R.A. Gray, Effects of irradiation on the fracture toughness of fbr structural materials, J. Nucl. Mater. 148 (1987) 194-203.

[158] Y. Dai, X.J. Jia, K. Farrell, Mechanical properties of modified 9Cr-1Mo (T91) irradiated at [less-than-or-equals, slant]300 ${ }^{\circ} \mathrm{C}$ in SINQ Target-3, J. Nucl. Mater. 318 (2003) 192-199.

[159] E. Wakai, S. Jitsukawa, H. Tomita, K. Furuya, M. Sato, K. Oka, et al., Radiation hardening and -embrittlement due to He production in $\mathrm{F} 82 \mathrm{H}$ steel irradiated at $250{ }^{\circ} \mathrm{C}$ in JMTR, J. Nucl. Mater. 343 (2005) 285-296.

[160] T.S. Byun, M.B. Toloczko, T.A. Saleh, S.A. Maloy, Irradiation dose and temperature dependence of fracture toughness in high dose HT9 steel from the fuel duct of FFTF, J. Nucl. Mater. 432 (2013) 1-8.

[161] T.L. Anderson, Fracture mechanics: fundamentals and applications, CRC Press, Boca Raton, FL, 2005.

[162] A.M. Cuitiño, M. Ortiz, Ductile fracture by vacancy condensation in f.c.c. single crystals, Acta Mater. 44 (1996) 427-436.

[163] R. Raj, M.. Ashby, Intergranular fracture at elevated temperature, Acta Met. 23 (1975) 653-666.

[164] R. Raj, Nucleation of cavities at second phase particles in grain boundaries, Acta Met. 26 (1978) 995-1006.

[165] M. Yoo, H. Trinkaus, Crack and cavity nucleation at interfaces during creep, Met. Mater. Trans. 14 (1983) 547-561.

[166] J.P. Hirth, W.D. Nix, Analysis of cavity nucleation in solids subjected to external and internal stresses, Acta Met. 33 (1985) 359-368.

[167] I.-W. Chen, A.S. Argon, Creep cavitation in 304 stainless steel, Acta Met. 29 (1981) 1321-1333.

[168] J.S. Wang, J.J. Stephens, W.D. Nix, A statistical analysis of cavity nucleation at particles in grain boundaries, Acta Met. 33 (1985) 1009-1021.

[169] H.R. Brager, F.A. Garner, G.L. Guthrie, The effect of stress on the microstructure of neutron irradiated type 316 stainless steel, J. Nucl. Mater. 66 (1977) 301-321. 
[170] F.A. McClintock, A criterion for ductile fracture by the growth of holes, J. Appl. Mech. 35 (1968) 363.

[171] J.R. Rice, D.M. Tracey, On the ductile enlargement of voids in triaxial stress fields, J. Mech. Phys. Solids. 17 (1969) 201-217.

[172] A.L. Gurson, Continuum theory of ductile rupture by void nucleation and growth: Part IYield criteria and flow rules for porous ductile media, J. Eng. Mater. Technol. 99 (1977) 215.

[173] V. Tvergaard, Influence of voids on shear band instabilities under plane strain conditions, Int. J. Fract. 17 (1981) 389-407.

[174] V. Tvergaard, On localization in ductile materials containing spherical voids, Int. J. Fract. 18 (1982) 237-252.

[175] V. Tvergaard, A. Needleman, Analysis of the cup-cone fracture in a round tensile bar, Acta Met. 32 (1984) 157-169.

[176] A.A. Benzerga, J.-B. Leblond, Ductile Fracture by Void Growth to Coalescence, in: Adv. Appl. Mech., Elsevier, 2010: pp. 169-305.

[177] A.A. Benzerga, Micromechanics of coalescence in ductile fracture, J. Mech. Phys. Solids. 50 (2002) 1331-1362.

[178] A.A. Benzerga, J. Besson, A. Pineau, Anisotropic ductile fracture: Part II: theory, Acta Mater. 52 (2004) 4639-4650.

[179] J. Besson, Continuum Models of Ductile Fracture: A Review, Int. J. Damage Mech. 19 (2010) 3-52.

[180] D. Hull, D.E. Rimmer, The growth of grain-boundary voids under stress, Philos. Mag. 4 (1959) 673-687.

[181] M.V. Speight, J.E. Harris, The Kinetics of Stress-Induced Growth of Grain-Boundary Voids, Met. Sci. 1 (1967) 83-85.

[182] J. Weertman, Theory of high temperature intercrystalline fracture under static or fatigue loads, with or without irradiation damage, Met. Mater. Trans. B. 5 (1974) 1743-1751.

[183] I.-W. Chen, A.S. Argon, Diffusive growth of grain-boundary cavities, Acta Met. 29 (1981) 1759-1768.

[184] A.C.F. Cocks, M.F. Ashby, On creep fracture by void growth, Prog. Mater. Sci. 27 (1982) 189-244.

[185] A. Needleman, J.. Rice, Plastic creep flow effects in the diffusive cavitation of grain boundaries, Acta Met. 28 (1980) 1315-1332.

[186] R.E. Stoller, G.R. Odette, A model based fission-fusion correlation of cavity swelling in stainless steel, J. Nucl. Mater. 104 (1981) 1361-1365.

[187] F.D. Fischer, J. Svoboda, Void growth due to vacancy supersaturation - A nonequilibrium thermodynamics study, Scr. Mater. 58 (2008) 93-95.

[188] E.A. Repetto, M. Ortiz, A micromechanical model of cyclic deformation and fatiguecrack nucleation in f.c.c. single crystals, Acta Mater. 45 (1997) 2577-2595. 
[189] J. Lemaitre, A course on damage mechanics, Springer-Verlag, Berlin, 1992.

[190] L.K. Mansur, Theory and experimental background on dimensional changes in irradiated alloys, J. Nucl. Mater. 216 (1994) 97-123.

[191] R.W. Balluffi, A.V. Granato, F. R. N. Nabarro, Editor, Dislocations in Solids, Vol. 4, North Holland, Amsterdam, 1979.

[192] M. Gologanu, J.-B. Leblond, J. Devaux, Approximate models for ductile metals containing non-spherical voids - Case of axisymmetric prolate ellipsoidal cavities, J. Mech. Phys. Solids. 41 (1993) 1723-1754.

[193] V.P. Bennett, D.L. McDowell, Crack tip displacements of microstructurally small surface cracks in single phase ductile polycrystals, Eng. Fract. Mech. 70 (2003) 185-207.

[194] G.M. Castelluccio, D.L. McDowell, Assessment of small fatigue crack growth driving forces in single crystals with and without slip bands, Int. J. Fract. 176 (2012) 49-64.

[195] C.F. Shih, Relationships between the J-integral and the crack opening displacement for stationary and extending cracks, J. Mech. Phys. Solids. 29 (1981) 305-326.

[196] E.E. Underwood, The mathematical foundations of quantitative stereology, in: Ster. Quant. Met. ASTM STP 504, American Soceity for Testing and Materials, Atlantic City, NJ, 1971: pp. 3-38.

[197] H. Mehrer, M. Lübbehusen, Self-Diffusion along Dislocations and in the Lattice of alpha-Iron, in: Defect Diffus. Forum, Trans Tech Publ, 1991: pp. 591-604.

[198] Y. Shima, Y. Ishikawa, H. Nitta, Y. Yamazaki, K. Mimura, M. Isshiki, et al., Selfdiffusion along dislocations in ultra high purity iron, Mater. Trans. 43 (2002) 173-177.

[199] F.A. McClintock, Plasticity aspects of fracture, in: Fract. Adv. Treatise, Academic Press, New York, 1971: pp. 47-225.

[200] Z. Li, W. Guo, The influence of plasticity mismatch on the growth and coalescence of spheroidal voids on the bimaterial interface, Int. J. Plast. 18 (2002) 249-279.

[201] R. Becker, Effects of strain localization on surface roughening during sheet forming, Acta Mater. 46 (1998) 1385-1401.

[202] O. Akourri, M. Louah, A. Kifani, G. Gilgert, G. Pluvinage, The effect of notch radius on fracture toughness JIc, Eng. Fract. Mech. 65 (2000) 491-505.

[203] R.O. Ritchie, R.M. Horn, Further considerations on the inconsistency in toughness evaluation of AISI 4340 steel austenitized at increasing temperatures, Met. Trans. 9 (1978) 331-341.

[204] M. Yoda, The effect of the notch root radius on the J-integral fracture toughness under modes I, II and III loadings, Eng. Fract. Mech. 26 (1987) 425-431.

[205] R. Damani, R. Gstrein, R. Danzer, Critical notch-root radius effect in SENB-S fracture toughness testing, J. Eur. Ceram. Soc. 16 (1996) 695-702.

[206] T. Fett, Influence of a finite notch root radius on fracture toughness, J. Eur. Ceram. Soc. 25 (2005) 543-547. 
[207] T. Nishida, Y. Hanaki, G. Pezzotti, Effect of Notch-Root Radius on the Fracture Toughness of a Fine-Grained Alumina, J. Am. Ceram. Soc. 77 (1994) 606-608.

[208] R.O. Ritchie, B. Francis, W.L. Server, Evaluation of toughness in AISI 4340 alloy steel austenitized at low and high temperatures, Met. Trans. 7 (1976) 831-838.

[209] T.R. Wilshaw, C.A. Rau, A.S. Tetelman, A general model to predict the elastic-plastic stress distribution and fracture strength of notched bars in plane strain bending, Eng. Fract. Mech. 1 (1968) 191-211.

[210] G.R. Odette, T. Yamamoto, H.J. Rathbun, M.Y. He, M.L. Hribernik, J.W. Rensman, Cleavage fracture and irradiation embrittlement of fusion reactor alloys: mechanisms, multiscale models, toughness measurements and implications to structural integrity assessment, J. Nucl. Mater. 323 (2003) 313-340.

[211] J. Garnier, Y. Bréchet, M. Delnondedieu, C. Pokor, P. Dubuisson, A. Renault, et al., Irradiation creep of SA 304L and CW 316 stainless steels: Mechanical behaviour and microstructural aspects. Part I: Experimental results, J. Nucl. Mater. 413 (2011) 63-69.

[212] C. Xu, G.S. Was, In situ proton irradiation creep of ferritic-martensitic steel T91, J. Nucl. Mater. 441 (2013) 681-687.

[213] J. Garnier, Y. Bréchet, M. Delnondedieu, A. Renault, C. Pokor, P. Dubuisson, et al., Irradiation creep of SA 304L and CW 316 stainless steels: Mechanical behaviour and microstructural aspects. Part II: Numerical simulation and test of SIPA model, J. Nucl. Mater. 413 (2011) 70-75.

[214] J. Chen, P. Jung, J. Henry, Y. de Carlan, T. Sauvage, F. Duval, et al., Irradiation creep and microstructural changes of ODS steels of different Cr-contents during helium implantation under stress, J. Nucl. Mater. 437 (2013) 432-437.

[215] M. Toloczko, F. Garner, Stress and Temperature Dependence of Irradiation Creep of Selected FCC and BCC Steels at Low Swelling, J. ASTM Int. 1 (2004) 73-85.

[216] S.. Bruemmer, E.. Simonen, P.. Scott, P.. Andresen, G.. Was, J.. Nelson, Radiationinduced material changes and susceptibility to intergranular failure of light-water-reactor core internals, J. Nucl. Mater. 274 (1999) 299-314.

[217] P.T. Heald, J.E. Harbottle, Irradiation creep due to dislocation climb and glide, J. Nucl. Mater. 67 (1977) 229-233.

[218] J.P. Foster, K. Bunde, D.L. Porter, Irradiation creep of annealed 304L stainless steel at low dose levels, J. Nucl. Mater. 317 (2003) 167-174.

[219] A. Causey, In-Reactor Stress Relaxation of Zirconium Alloys, in: J. Schemel, H. Rosenbaum (Eds.), Zircon. Nucl. Appl., ASTM International, 100 Barr Harbor Drive, PO Box C700, West Conshohocken, PA 19428-2959, 1974: pp. 263-263-11.

[220] A.R. Causey, F.J. Butcher, S.A. Donohue, Measurement of irradiation creep of zirconium alloys using stress relaxation, J. Nucl. Mater. 159 (1988) 101-113.

[221] J. Chen, P. Jung, W. Hoffelner, Irradiation creep of candidate materials for advanced nuclear plants, J. Nucl. Mater. 441 (2013) 688-694. 
[222] V. Vitek, Structure of dislocation cores in metallic materials and its impact on their plastic behaviour, Prog. Mater. Sci. 36 (1992) 1-27.

[223] M.S. Duesbery, V. Vitek, Plastic anisotropy in b.c.c. transition metals, Acta Mater. 46 (1998) 1481-1492.

[224] V. Vitek, Core structure of screw dislocations in body-centred cubic metals: relation to symmetry and interatomic bonding, Philos. Mag. 84 (2004) 415-428.

[225] V. Vitek, M. Mrovec, J.L. Bassani, Influence of non-glide stresses on plastic flow: from atomistic to continuum modeling, Mater. Sci. Eng. 365 (2004) 31-37.

[226] R. Gröger, V. Racherla, J.L. Bassani, V. Vitek, Multiscale modeling of plastic deformation of molybdenum and tungsten: II. Yield criterion for single crystals based on atomistic studies of glide of screw dislocations, Acta Mater. 56 (2008) 5412-5425.

[227] V. Vitek, M. Mrovec, R. Gröger, J.L. Bassani, V. Racherla, L. Yin, Effects of non-glide stresses on the plastic flow of single and polycrystals of molybdenum, Mater. Sci. Eng. 387-389 (2004) 138-142.

[228] J. Chaussidon, M. Fivel, D. Rodney, The glide of screw dislocations in bcc Fe: Atomistic static and dynamic simulations, Acta Mater. 54 (2006) 3407-3416.

[229] A. Koester, A. Ma, A. Hartmaier, Atomistically informed crystal plasticity model for body-centered cubic iron, Acta Mater. 60 (2012) 3894-3901.

[230] G.J. Irwin, F. Guiu, P.L. Pratt, The influence of orientation on slip and strain hardening of molybdenum single crystals, Phys. Status Solidi. 22 (1974) 685-698.

[231] R. Gröger, A.G. Bailey, V. Vitek, Multiscale modeling of plastic deformation of molybdenum and tungsten: I. Atomistic studies of the core structure and glide of $1 / 2<1 \quad 1 \quad 1\rangle$ screw dislocations at $0 \mathrm{~K}$, Acta Mater. 56 (2008) 5401-5411.

[232] C.. Statham, D. Vesely, J.. Christian, Slip in single crystals of niobium-molybdenum alloys deformed in compression, Acta Met. 18 (1970) 1243-1252.

[233] C.J. Bolton, G. Taylor, Anomalous slip in high-purity niobium single crystals deformed at $77^{\circ} \mathrm{K}$ in tension, Philos. Mag. 26 (1972) 1359-1376.

[234] J. Christian, Some surprising features of the plastic deformation of body-centered cubic metals and alloys, Met. Mater. Trans. 14 (1983) 1237-1256.

[235] M.H.A. Nawaz, B.L. Mordike, Slip Geometry of Tantalum and Tantalum Alloys, Phys. Status Solidi. 32 (1975) 449-458.

[236] T. Yalcinkaya, W.A.M. Brekelmans, M.G.D. Geers, BCC single crystal plasticity modeling and its experimental identification, Model. Simul. Mater. Sci. Eng. 16 (2008) 085007.

[237] T. Buchheit, C. Battaile, C. Weinberger, E. Holm, Multi-scale modeling of lowtemperature deformation in b.c.c. metals, JOM J. Miner. Met. Mater. Soc. 63 (2011) 33-36.

[238] C.R. Weinberger, C.C. Battaile, T.E. Buchheit, E.A. Holm, Incorporating atomistic data of lattice friction into BCC crystal plasticity models, Int. J. Plast. 37 (2012) 16-30. 
[239] H. Lim, C.R. Weinberger, C.C. Battaile, T.E. Buchheit, Application of generalized nonSchmid yield law to low-temperature plasticity in bcc transition metals, Model. Simul. Mater. Sci. Eng. 21 (2013) 045015.

[240] D. Caillard, Kinetics of dislocations in pure Fe. Part I. In situ straining experiments at room temperature, Acta Mater. 58 (2010) 3493-3503.

[241] D. Caillard, Kinetics of dislocations in pure Fe. Part II. In situ straining experiments at low temperature, Acta Mater. 58 (2010) 3504-3515.

[242] M.R. Gilbert, S. Queyreau, J. Marian, Stress and temperature dependence of screw dislocation mobility in $\alpha$-Fe by molecular dynamics, Phys. Rev. B. 84 (2011) 174103.

[243] D. Brunner, J. Diehl, Strain-Rate and Temperature Dependence of the Tensile Flow Stress of High-Purity $\alpha$-Iron above $250 \mathrm{~K}$ (Regime I) Studied by Means of StressRelaxation Tests, Phys. Status Solidi. 124 (1991) 155-170.

[244] D. Brunner, J. Diehl, Temperature and Strain-Rate Dependence of the Tensile Flow Stress of High-Purity $\alpha$-Iron below 250 K. I. Stress/Temperature Regime III, Phys. Status Solidi. 124 (1991) 455-464.

[245] T. Takeuchi, Orientation Dependence of Work-Hardening in Iron Single Crystals, Jpn. J. Appl. Phys. 8 (1969) 320-328.

[246] A.S. Argon, Strengthening mechanisms in crystal plasticity, Oxford University Press Oxford, 2008.

[247] J.J. Adams, D.S. Agosta, R.G. Leisure, H. Ledbetter, Elastic constants of monocrystal iron from 3 to $500 \mathrm{~K}$, J. Appl. Phys. 100 (2006) 113530.

[248] W.. Spitzig, A.. Keh, Orientation dependence of the strain-rate sensitivity and thermally activated flow in iron single crystals, Acta Met. 18 (1970) 1021-1033.

[249] D. Brunner, J. Diehl, Temperature and Strain-Rate Dependence of the Tensile Flow Stress of High-Purity $\alpha$-Iron below 250 K II. Stress/Temperature Regime II and Its Transitions to Regimes I and III, Phys. Status Solidi. 125 (1991) 203-216.

[250] W. Spitzig, A. Keh, The role of internal and effective stresses in the plastic flow of iron single crystals, Met. Mater. Trans. B. 1 (1970) 3325-3331.

[251] S. Naamane, G. Monnet, B. Devincre, Low temperature deformation in iron studied with dislocation dynamics simulations, Int. J. Plast. 26 (2010) 84-92.

[252] D.. Stein, J.. Low Jr., Effects of orientation and carbon on the mechanical properties of iron single crystals, Acta Met. 14 (1966) 1183-1194.

[253] B. Šesták, N. Zárubová, Asymmetry of Slip in Fe-Si Alloy Single Crystals, Phys. Status Solidi B. 10 (1965) 239-250.

[254] S. Takeuchi, E. Furubayashi, T. Taoka, Orientation dependence of yield stress in 4.4\% silicon iron single crystals, Acta Met. 15 (1967) 1179-1191. 